\title{
JET PRODUCTION IN THE CENTRAL RAPIDITY REGION \\ IN 1.8 TEV PROTON AND ANTIPROTON COLLISIONS
}

\author{
BY \\ YEONG-DONG TSAI \\ JUNE, 1989
}


THE UNIVERSITY OF CHICAGO

JET PRODUCTION IN THE CENTRAL RAPIDITY REGION

IN 1.8 TEV PROTON AND ANTIPROTON COLLISIONS

\author{
A DISSERTATION SUBMITTED TO \\ THE FACULTY OF THE DIVISION OF THE PHYSICAL SCIENCES \\ IN CANDIDACY FOR THE DEGREE OF \\ DOCTOR OF PHILOSOPHY \\ DEPARTMENT OF PHYSICS \\ BY \\ YEONG-DONG TSAI
}

CHICAGO, ILLINOIS

JUNE, 1989 


\section{ACKNOWLEDGEMENTS}

I thank every member of the CDF collaboration who has spent many years of his (her) life to make this experiment a success.

In particular I thank the CDF Jet Group for making this analysis possible. I am especially grateful to Steve Behrends, Stephen Kuhlmann and John Huth, whose beautiful work had a direct impact on this analysis. I also have learned a great deal about the UA2 Pt balancing technique from Richard St. Denis.

At The University of Chicago, where I grew from a wishful youth to a middle-aged man, I am most indebted to my thesis advisor, Henry J. Frisch, who has been my support in many ways for so many years. I cannot imagine what I would be like if he had not been my advisor. Professor Melvyn Shochet is an example of the brilliant physicists in this great academy. Henry and Mel have shown me the special qualities it takes to be professors at Chicago. I also thank the members of my thesis committee, Robert Geroch, Reinhard Dehme and Frank Merritt for overseeing my research. Frank's criticism has been particularly helpful.

Myron Campbell has been a good source for help since the time I joined the Chicago CDF group. His ability to quickly look into and solve a problem is truly admirable. George Redlinger and Frederick Snider are my fellow graduate students in the same group and same office, and they have supplied me information about the goings-on in CDF and in the high energy physics community. This memory of growing-up together will be carried to everywhere I go. Sunil Somalwar, as another student who came from a distant country, is always willing to help me with his resourceful knowledge. There are many others from whom I have benefitted, and they are: Harold Sanders, Joe Ting, Dan Amidei, Carla Grosso-Pilcher, Jay Hauser, Tony Liss, Paul Derwent, and Aaron Roodman. Paul Derwent read some parts of my draft of this thesis and corected several English errors, which $I$ was not aware of for so many years.

I thank Kathy Visak for much help during these years, and Marypat Sharer for making me feel at home. 
I thank E. Eichten, J. Bagger, C. Schmidt and S. King for providing me with the programs needed to do the theoretical calculations.

I dedicate this thesis to my parents, to my brother and sisters, to my future (possibly) wife.

This work is supported by the U.S. Department of Energy; the National Science Foundation (NSF Contract PHY88-23033); Istituto Nazionale di. Fisica Nucleare, Italy; and Ministry of Science, Culture and Education of Japan. 


\section{THE CDF COLLABORATION}

F. Abe, ${ }^{(16)}$ D. Amidei, (3) G. Apollinari, (11) G. Ascoli, ${ }^{(7)}$ M. Atac, ${ }^{(4)}$ P. Auchincloss, ${ }^{(14)}$ A. R. Baden, ${ }^{(6)}$ A. Barbaro-Galtieri, ${ }^{(9)}$ V. E. Barnes, ${ }^{(12)}$ F. Bedeschi, ${ }^{(11)}$ S. Belforte, ${ }^{(11)}$ G. Bellettini, (II) J. Bellinger, (17) J. Bensinger, ${ }^{(2)}$ A. Beretvas, ${ }^{(14)}$ P. Berge, ${ }^{(4)}$ S. Bertolucei, ${ }^{(5)}$ S. Bhadra, ${ }^{(7)}$ M. Binkley, ${ }^{(4)}$ R. Blair, (1) C. Blocker, ${ }^{(2)}$ J. Bofill, ${ }^{(1)}$ A. W. Booth, ${ }^{(1)}$ G. Brandenburg, ${ }^{(6)}$ D. Brown, ${ }^{(6)}$ A. Byon, ${ }^{(12)}$ K. L. Byrum, ${ }^{(17)}$ M. Campbell, ${ }^{(3)}$ R. Carey, ${ }^{(6)}$ W. Carithers, ${ }^{(9)}$ D. Carlsmith, ${ }^{(17)}$ J. T. Carroll, ${ }^{(4)}$ R. Cashmore, (4) F. Cervelli, ${ }^{(11)}$ K. Chadwick, (4,12) T. Chapin, ${ }^{(13)}$ G. Chiarelli, (11) W. Chinowsky, ${ }^{(9)}$ S. Cihangir, (15) D. Cline, ${ }^{(17)}$ D. Connor, ${ }^{(10)}$ M. Contreras, ${ }^{(2)}$ J. Cooper, ${ }^{(4)}$ M. Cordelli, ${ }^{(5)}$ M. Curatolo, ${ }^{(5)}$ C. Day, ${ }^{(4)}$ R. DelFabbro, ${ }^{(11)}$ M. Dell'Orso, ${ }^{(11)}$ L. DeMortier, ${ }^{(2)}$ T. Devlin, ${ }^{(14)}$ D. DiBitonto, ${ }^{(15)}$ R. Diebold, ${ }^{(1)}$ F. Dittus, ${ }^{(4)}$ A. DiVirgilio, ${ }^{(11)}$ J. E. Elias, ${ }^{(4)}$ R. Ely, ${ }^{(9)}$ S. Errede, ${ }^{(7)}$ B. Esposito, ${ }^{(5)}$ A. Feldman, ${ }^{(6)}$ B. Flaugher, ${ }^{(14)}$ E. Focardi, ${ }^{(11)}$ G. W. Foster, ${ }^{(4)}$ M. Franklin, ${ }^{(6,7)}$ J. Freeman, ${ }^{(4)}$ H. Frisch, ${ }^{(3)}$ Y. Fukui, ${ }^{(8)}$ A. F. Garfinkel, ${ }^{(12)}$ P. Giannetti, ${ }^{(11)}$ N. Giokaris, ${ }^{(13)}$ P. Giromini, ${ }^{(5)}$ L. Gladney, ${ }^{(10)}$ M. Gold, ${ }^{(9)}$ K. Goulianos, ${ }^{(13)}$ C. Grosso-Pilcher, ${ }^{(3)}$ C. Haber, ${ }^{(9)}$ S. R. Hahn, ${ }^{(10)}$ R. Handler, ${ }^{(17)}$ R. M. Harris, ${ }^{(9)}$ J. Hauser, ${ }^{(3)}$ T. Hessing, ${ }^{(15)}$ R. Hollebeek, ${ }^{(10)}$ L. Holloway, ${ }^{(7)}$ P. Hu, ${ }^{(14)}$ B. Hubbard, ${ }^{(9)}$ P. Hurst, ${ }^{(7)}$ J. Huth, ${ }^{(4)}$ H. Jensen, ${ }^{(4)}$ R. P. Johnson, ${ }^{(4)}$ U. Joshi, ${ }^{(14)}$ R. W. Kadel, ${ }^{(4)}$ T. Kamon, ${ }^{(15)}$ S. Kanda, (16) D. A. Kardelis, ${ }^{(7)}$ I. Karliner, ${ }^{(7)}$ E. Kearns, ${ }^{(6)}$ R. Kephart, ${ }^{(4)}$ P. Kesten, ${ }^{(2)}$ H. Keutelian, ${ }^{(7)}$ S. Kim, ${ }^{(16)}$ L. Kirsch, ${ }^{(2)}$ K. Kondo, ${ }^{(16)}$ U. Kruse, ${ }^{(7)}$ S. E. Kuhlmann, ${ }^{(12)}$ A. T. Laasanen, ${ }^{(12)}$ W. Li, ${ }^{(1)}$ T. Lias, ${ }^{(3)}$ N. Lockyer, ${ }^{(10)}$ F. Marchetto, ${ }^{(15)}$ R. Markelof,, ${ }^{(17)}$ L. A. Markosky, ${ }^{(17)}$ P. McIntyre, ${ }^{(15)}$ A. Menzione, ${ }^{(11)}$ T. Meyer, ${ }^{(15)}$ S. Mikamo, ${ }^{(1)}$ M. Miller, ${ }^{(10)}$ T. Mimashi, ${ }^{(16)}$ S. Miscetti, ${ }^{(5)}$ M. Mishina, ${ }^{(8)}$ S. Miyashita, ${ }^{(16)}$ N. Mondal, ${ }^{(17)}$ S. Mori, ${ }^{(16)}$ Y. Morita, ${ }^{(16)}$ A. Mukherjee, ${ }^{(4)}$ C. Newman-Holmes, ${ }^{(1)}$ L. Nodulman, ${ }^{(1)}$ R. Paoletti, ${ }^{(11)}$ A. Para, ${ }^{(4)}$ J. Patrick, ${ }^{(4)}$ T. J. Phillips, ${ }^{(6)}$ H. Piekarz, ${ }^{(2)}$ R. Plunkett, ${ }^{(13)}$ L. Pondrom, ${ }^{(17)}$ J. Proudfoot, (1) G. Punzi, (11) D. Quarrie, ${ }^{(4)}$ K. Ragan, ${ }^{(10)}$ G. Redlinger, ${ }^{(3)}$ J. Rhoades, ${ }^{(17)}$ F. Rimondi, ${ }^{(4)}$ L. Ristori, ${ }^{(1)}$ T. Rohaly, ${ }^{(10)}$ A. Roodman, ${ }^{(3)}$ A. Sansoni, ${ }^{(5)}$ R. Sard, ${ }^{(7)}$ V. Scarpine, ${ }^{(7)}$ P. Schlabach, ${ }^{(7)}$ E. E. Schmidt, (4) P. Schoessow, (1) M. H. Schub, ${ }^{(12)}$ R. Schwitters, ${ }^{(6)}$ A. Scribano, ${ }^{(11)}$ S. Segler, ${ }^{(1)}$ M. Sekiguchi, ${ }^{(16)}$ P. Sestini, ${ }^{(11)}$ M. Shapiro, ${ }^{(6)}$ M. Sheaff, ${ }^{(17)}$ M. Shibata, ${ }^{(16)}$ M. Shochet, ${ }^{(3)}$ J. Siegrist, ${ }^{(9)}$ P. Sinervo, ${ }^{(10)}$ J. Skarha, ${ }^{(17)}$ D. A. Smith, ${ }^{(7)}$ F. D. Snider, ${ }^{(3)}$ R. St. Denis, ${ }^{(6)}$ A. Stefanini, (11) Y. Takaiwa, ${ }^{(16)}$ K. Takikawa, ${ }^{(16)}$ S. Tarem, ${ }^{(3)}$ D. Theriot, ${ }^{(4)}$ A. Tollestrup, ${ }^{(4)}$ G. Tonelli, ${ }^{(11)}$ Y. Tsay, ${ }^{(3)}$ F. Ukegawa, ${ }^{(16)}$ D. Underwood, ${ }^{(1)}$ R. Vidal, ${ }^{(4)}$ R. G. Wagner, ${ }^{(1)}$ R. L. Wagner, ${ }^{(4)}$ J. Walsh, (10) T. Watts, ${ }^{(14)}$ R. Webb, ${ }^{(15)}$ T. Westhusing, ${ }^{(7)}$ S. White, ${ }^{(13)}$ A. Wicklund, ${ }^{(1)}$ H. H. Williams ${ }^{(10)}$ T. Yamanouchi, ${ }^{(4)}$ A. Yamashita, ${ }^{(16)}$ K. Yasuoka, ${ }^{(16)}$ G. P. Yeh, ${ }^{(4)}$ J. Yoh, ${ }^{(4)}$ and F. Zetti, (1)

(1) Argonne National Laboratory, Argonne, Illinois 60439

(2) Brandeis University, Waltham, Massachusetts 02254

(3) University of Chicago, Chicago, Illinois 60637

(1) Fermi National Accelerator Laboratory, Batavia, Illimois 60510

(3) Laboratori Nazionall di Frascati, Istituto Nazionale di Fisica Nucleare, Frascati, Italy

${ }^{(6)}$ Harvard University, Cambridge, Massachusetts 02138

(") Universtty of Illinois, Urbana, Illinois 61801

(1) National Labaratory for High Energy Physics (KEX), Tsukuba-gun, Ibaraki-ken 309, Japan

(') Lawrence Berkeley Laboratory. Berkeley. Callformia 94720

${ }^{(10)}$ University of Pennsylvania, Philadelphia, Pennsylvania 19104

(11'Istituto Nazionale di Fisica Nucleare, University and Scuola Normale Superiore of Pisa. Pisa. Italy

(12) Purdue University. West Lafayette, Indiana 47907

(13) Rockefeller University. New York. New York 10021

${ }^{(14)}$ Rutgers University. Piscataway, New Jersey 08854

${ }^{(13)}$ Texas A\&M University, College Station, Texas 77843

(16) University of Tsukuba, lbaraki 30S, Japan

(1" University of Wisconsin, Madison, Wisconsin 53706 
TABLE OF CONTENTS

ACKNOWLEDGEMENTS $\ldots \ldots \ldots \ldots \ldots \ldots \ldots \ldots \ldots \ldots \ldots \ldots \ldots \ldots \ldots \ldots \ldots \ldots$

THE CDF COLLABORATION $\ldots \ldots \ldots \ldots \ldots \ldots \ldots \ldots \ldots \ldots \ldots \ldots \ldots \ldots \ldots$ iv

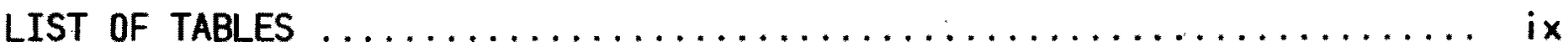

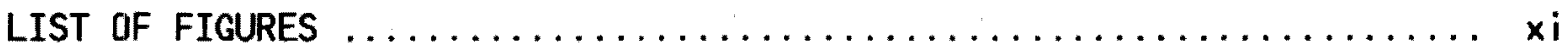

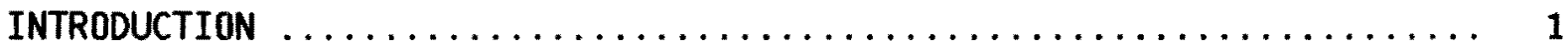

Chapter

1. JET PRODUCTION IN HADRON COLLISIONS $\ldots \ldots \ldots \ldots \ldots \ldots \ldots \ldots \ldots \ldots$

1.1 The Story of Quantum Chromodynamics .............. 4

1.2 An Asymptotically Free Theory $\ldots \ldots \ldots \ldots \ldots \ldots \ldots \ldots \ldots$

1.3 The Process of Hadron Collisions ................ 7

1.4 QCD Evolution of Structure Functions .............. 8

1.5 Hadronization of Quarks and Gluons ............... 10

1.6 Beam Jets and Initial State Radiation ............... 11

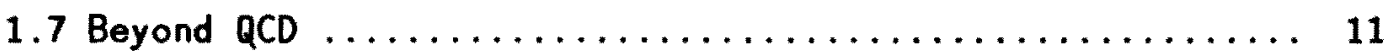

1.7.1 Are Quarks Composite? .................. 11

1.7 .2 Is QCD Chiral? ........................ 12

2. THE COLLIDER DETECTOR AT FERMILAB (CDF) $\ldots \ldots \ldots \ldots \ldots \ldots \ldots$

2.1 Overview ............................ 16

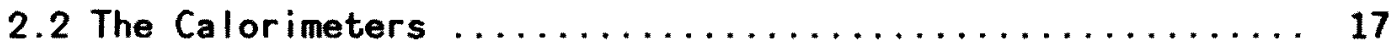

2.2.1 The Central EM Calorimeters (CEM) ............ 19

2.2.2 The Central and Endwall Hadron Calorimeters (CHA and WHA) ......................... 20

2.2 .3 The Calibration of CEM .................. 21

2.2.4 The Calibration of CHA and WHA ............. 22

2.2.5 The Plug and Forward Gas Calorimeters:

PEM, PHA, FEM and FHA ............... 23

2.3 The Vertex Time Projection Chambers (VTPC) $\ldots \ldots \ldots \ldots \ldots .25$ 
3.1 Front End Electronics (RABBIT) $\ldots \ldots \ldots \ldots \ldots \ldots \ldots \ldots, 41$

3.2 The Chicago Trigger ........................ 43

3.2.1 The Level-1 Calorimetry Trigger ............. 44

3.2.2 Other Level-1 Triggers .................. 47

3.2.3 Final Decision Logic (FRED), Cross Points and Trigger Supervisor $\ldots \ldots \ldots \ldots \ldots \ldots \ldots \ldots, 48$

3.2.4 Trigger Timing Control (TC) $\ldots \ldots \ldots \ldots \ldots \ldots \ldots, 49$

3.3 The Online Data Acquisition System (DAQ) $\ldots \ldots \ldots \ldots \ldots, 49$

3.4 Network Organization ......................... 50

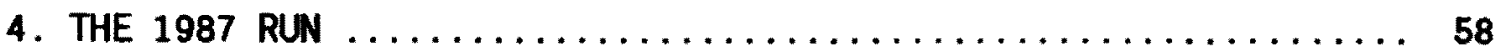

4.1 The Performance of $\overline{\mathrm{p}}$ Source and the Tevatron Collider ... 58

4.2 The CDF Performance ....................... 59

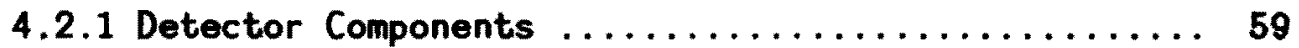

4.2.2 Monitoring and Calibration ............. 59

4.2 .3 Trigger ......................... 59

4.2.4 Data Taking Efficiency ............... 60

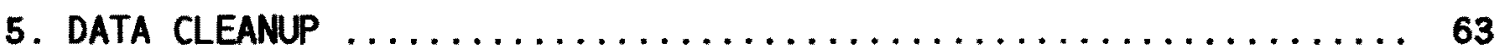

5.1 The Spikes in CEM, CHA and WHA ............... 63

5.2 Cosmic Rays and Stray Particles from the Main Ring ...... 64

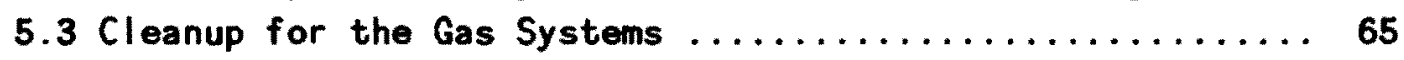

6. CLUSTERING ALGORITHMS, JET CHARACTERISTICS AND THE

COMPARISON OF DATA AND MONTE CARLO ............... 67

6.1 Clustering Algorithms $\ldots \ldots \ldots \ldots \ldots \ldots \ldots \ldots \ldots \ldots \ldots \ldots$

6.1 .1 JCLST2 and JCLST3 $\ldots \ldots \ldots \ldots \ldots \ldots \ldots \ldots \ldots \ldots \ldots 67$

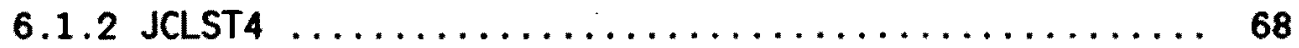

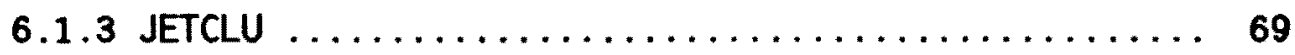

6.1.4 The Comparison and Performance Tests of

Clustering Algorithms ................. 70

6.2 Trigger Clustering Algorithm versus JETCLU .......... 71

6.3 Characteristics of Jets and the Comparison of

Data and Monte Carlo .................... 72 
7. MONTE CARLO STUDY OF JET ENERGY CORRECTION AND RESOLUTION ..... 95

7.1 The Sources of Jet Energy Loss ................ 95

7.2 Choosing ISAJET as the Event Generator ............. 97

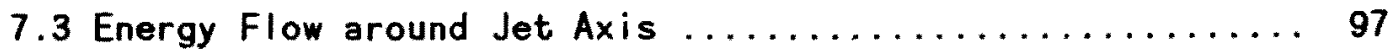

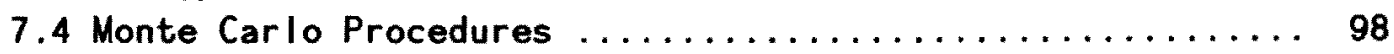

7.4 .1 Event Generation ........................ 98

7.4 .2 Detector Simulation .................. 100

7.4.3 Corrections and Resolutions ............. 100

7.5 How Energy Is Lost? . . . . . . . . . . . . . . . . . 103

7.6 Uncertainty in the Jet Energy Correction ............. 105

7.7 Uncertainty in the Jet Energy Resolution ............. 106

7.7.1 The UA2 Technique of Determining Pt Resolution

from Data ............................. 106

7.7.2 What Error Will Be Quoted for Resolution? ....... 109

8. INCLUSIVE JET ET AND DIJET MASS SPECTRA $\ldots \ldots \ldots \ldots \ldots \ldots \ldots$

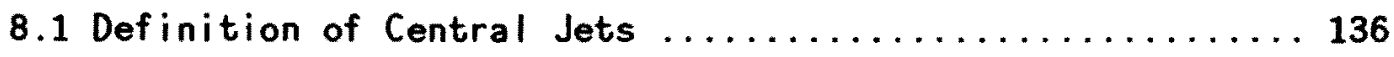

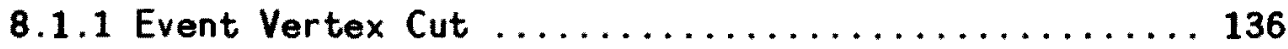

8.1.2 Pseudo-Rapidity Region ............... 137

8.2 Run Selection and Integrated Luminosity ............ 137

8.3 Criteria for Good Clusters ..................... 139

8.4 The Inclusive Jet Et and Dijet Mass Spectra .......... 140

8.4.1 Issue of Resolution Smearing ............ 141

8.4.2 The Systematic Uncertainties ............. 142

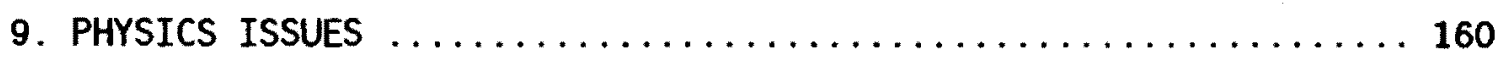

9.1 Comparison with Standard QCD ................ 160

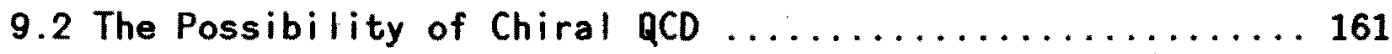

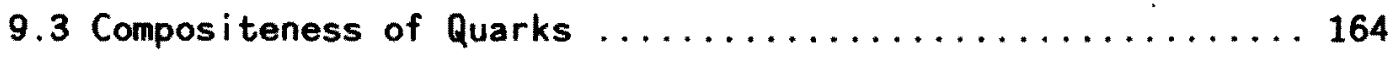

Appendix

A. THE RUNNING COUPLING CONSTANT AND STRUCTURE FUNCTIONS IN QCD $\ldots 185$

B. HADRONIZATION OF QUARKS AND GLUONS $\ldots \ldots \ldots \ldots \ldots \ldots \ldots \ldots \ldots \ldots$ 
C. BEAM JETS AND INITIAL STATE RADIATION $\ldots \ldots \ldots \ldots \ldots \ldots \ldots \ldots$

D. WHY ARE JETS ROUND IN $\eta-\phi$ SPACE? $\ldots \ldots \ldots \ldots \ldots \ldots \ldots \ldots \ldots \ldots$

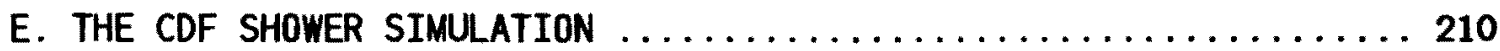

F. CHARACTERISTICS OF RUNS IN THE 1987 DATA $\ldots \ldots \ldots \ldots \ldots \ldots \ldots$

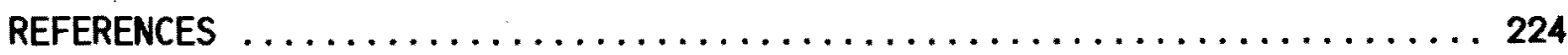




\section{LIST OF TABLES}

1 Properties of the Central Calorimeters .............. 27

2 Properties of the Plug and Forward Calorimeters $\ldots \ldots \ldots \ldots .28$

3 Properties of the Vertex Time Projection Chambers .......... 29

4 Comparison of the Clustering Algorithms $\ldots \ldots \ldots \ldots \ldots \ldots$

5 Real and Measured Jet Energy in Monte Carlo ............ 110

6 Real and Measured Jet Momenta in Monte Carlo ............ 111

7 Energy, Momentum and Dijet Mass Resolution in Monte Carlo ... 112

8 Formulae for Energy and Momentum Corrections ........... 113

9 Various Fits to the Energy, Momentum and Dijet Mass Resolutions .......................... 113

10 Various Cuts on Jets for Et Spectrum $\ldots \ldots \ldots \ldots \ldots \ldots \ldots$

11 Various Cuts on Events for $M_{J J}$ Spectrum $\ldots \ldots \ldots \ldots \ldots \ldots \ldots 143$

12 Resolution Unsmearing Factors for the Jet Et Spectrum ....... 144

13 Resolution Unsmearing Factors for the Dijet Mass Spectrum .... 145

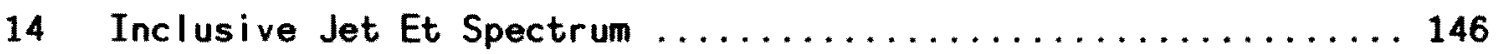

15 Inclusive Jet Pt Spectrum .................... 147

16 Dijet Mass Spectrum ...................... 148

17 The Comparison of QCD Predicted Pt Spectra and Data ........ 167

18 The Comparison of QCD Predicted $M_{J J}$ Spectra and Data ....... 167 
19 Probability of Agreement With Data for Various

Compositeness Scales ......................... 168

20 Characteristics of Runs in the 1987 Data ............ 217

$-$
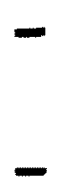


\section{LIST OF FIGURES}

1 Feynman Vertices Involving Axigluons $\ldots \ldots \ldots \ldots \ldots \ldots \ldots \ldots$

2 The Axigluon Contribution to the Lowest Order 2 Parton to 2 Parton Scattering ......................... 15

3 A Perspective View of the CDF Detector $\ldots \ldots \ldots \ldots \ldots \ldots \ldots$

4 A Cut-away View of the CDF Detector $\ldots \ldots \ldots \ldots \ldots \ldots \ldots \ldots$

5 The Assembly Building and Collision Hall for the Detector .... 32

6 Boundaries of the Calorimeter Towers on the $\eta-\phi$ Space ...... 33

7 A Central Calorimeter Wedge Showing the Light Collecting System for the CEM ....................... 34

8 A Cut-away View of the Central, Endwall and Plug Calorimeters Showing the Tower Boundaries in $\theta \ldots \ldots \ldots \ldots$

9 Wavelength Shifter Strips Collecting Light from Each CHA

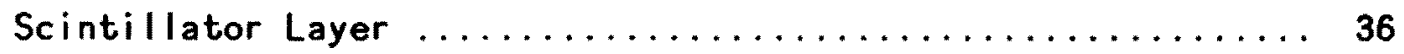

10 A Central Calorimeter Wedge Showing the Light Guide for a CHA Tower .............................. 36

11 Calibration Reproducibility for the CEM $\ldots \ldots \ldots \ldots \ldots \ldots \ldots$

12 Pulse Height Distribution for $50 \mathrm{GeV}$ Pions in a CHA Tower .... 38

13 Energy Resolution as a Function of Pion Energy for 3 CHA Towers $\ldots \ldots \ldots \ldots \ldots \ldots \ldots \ldots \ldots \ldots \ldots \ldots$

14 Exploded View of a PEM Layer $\ldots \ldots \ldots \ldots \ldots \ldots \ldots \ldots \ldots \ldots \ldots$

15 More Detailed View of the PEM Tube Array $\ldots \ldots \ldots \ldots \ldots \ldots . \ldots 39$

16 Cross Section of a Typical FEM (gas) Chamber ............ 40 
17 An Isometric View of Two VTPC Modules ............... 40

18 A Block Diagram of the CDF Readout System -. RABBIT ....... 52

19 A Charge-to-Voltage Convertor and

a Sample \& Hold Circuit $\ldots \ldots \ldots \ldots \ldots \ldots \ldots \ldots \ldots \ldots \ldots \ldots$

20 A Block Diagram of the CDF Level-1 and Level-2 Trigger System 53

21 A Schematic Showing the Operation of the Receive and Weight and Compare and Sum Boards .................... 54

22 One of the Beam-Beam Counter Arrays $\ldots \ldots \ldots \ldots \ldots \ldots \ldots$

23 The Data Acquisition (DAQ) Pipeline ............... 56

24 The Messages from/to the Buffer Manager Controlling the DAQ Pipeline ........................ 56

25 Crosspoint Modules $\ldots \ldots \ldots \ldots \ldots \ldots \ldots \ldots \ldots \ldots \ldots \ldots \ldots \ldots$

26 FASTBUS Network $\ldots \ldots \ldots \ldots \ldots \ldots \ldots \ldots \ldots \ldots \ldots \ldots \ldots$

27 Tevatron Peak Luminosity for the 1987 Run ............. 61

28 The Integrated Luminosity versus Time for the 1987 Run . . . . . 62

29 Times of Hits on the CHA Towers $\ldots \ldots \ldots \ldots \ldots \ldots \ldots \ldots \ldots \ldots$

30 Comparison of Jets as Found by the JETCLU and JCLST4 Algorithms $\ldots \ldots \ldots \ldots \ldots \ldots \ldots \ldots \ldots \ldots \ldots \ldots \ldots \ldots$

31 Comparison of Clusters as Found by the JETCLU and TRIGGER Algorithms (I) $\ldots \ldots \ldots \ldots \ldots \ldots \ldots \ldots \ldots \ldots$

32 Comparison of Clusters as Found by the JETCLU and

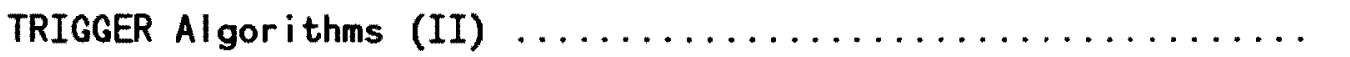

33 Comparison of Clusters as Found by the JETCLU and TRIGGER Algorithms (III) 
34 The Efficiency of the Trigger Level-2 Algorithm ......... 85

35 Et Weighted $\eta$ and $\phi$ Widths of Jets (Monte Carlo + Data) .... 88

36 The Number of Towers in Jets and the Tower Energy

Fractions $(M C+$ Data $) \ldots \ldots \ldots \ldots \ldots \ldots \ldots \ldots \ldots \ldots \ldots \ldots$

37 The Fraction of Jet Energy Contained in the

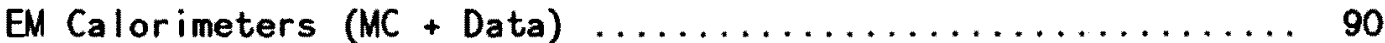

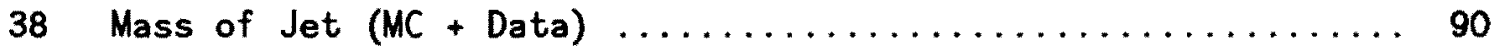

$39 \Delta \phi$ between the Two Leading Central Jets $(M C+$ Data $) \ldots \ldots \ldots 91$

40 Pt Ratio of the Two Leading Central Jets (MC + Data) ...... 91

41 Momentum of the Central Dijet System (MC + Data) $\ldots \ldots \ldots \ldots 9$

42 The Number of Clusters in Central Dijet Event (MC + Data) ... 93

43 Pt Ratio of the 3rd and 2nd Highest Pt Jets in Central

Dijet Event $(M C+$ Data $) \ldots \ldots \ldots \ldots \ldots \ldots \ldots \ldots \ldots \ldots$

44 Missing Pt in Central Dijet Event $(M C+$ Data $) \ldots \ldots \ldots \ldots . .94$

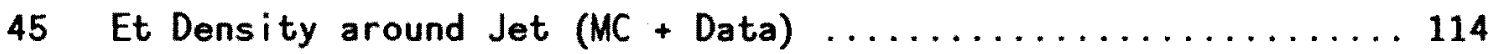

46 Et Content in Rings around Jet Center $(M C+$ Data $) \ldots \ldots \ldots 116$

47 Distributions of Et around Jet Center (Data) . . . . . . . 117

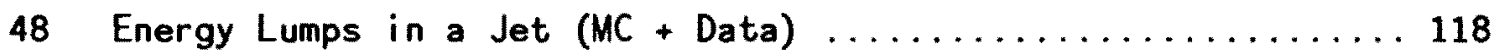

49 The Distributions of the Difference between the Measured and Real Jet Energies for Jets in Various Energy Ranges according to Monte Carlo ..................... 120

50 Measured versus True Jet Energies and Momenta ............ 125

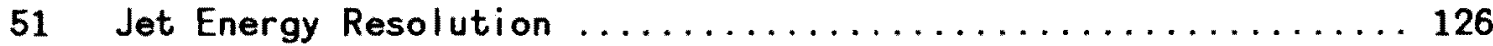


53 Jet Energy Resolutions with or without the Effects of Clustering and Underlying Energy

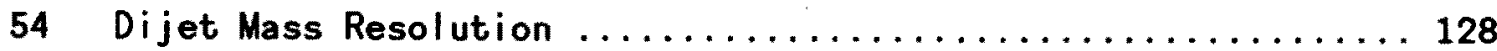

55 Jet Angular Resolution $\ldots \ldots \ldots \ldots \ldots \ldots \ldots \ldots \ldots \ldots \ldots \ldots \ldots$

56 Various Ways of Collecting Calorimeter Jet Energy (Used to Estimate How Energy Is Lost at Each Stage) .............. 129

57 Jet Energy Losses according to Monte Carlo ............. 130

58 Measured Jet Energy When the Parton Fragmentation, Calorimeter Response, etc Are Varied ............... 131

59 Uncertainty in the Jet Energy Correction ............. 132

60 The Definitions of $K_{\eta}$ and $K_{\xi}$ in the UA2 Pt Balancing Technique for Determining Jet Pt Resolution ............ 133

61 Resolutions in Monte Carlo Data Determined by the UA2 Technique ............................ 134

62 Resolutions in Real Data Determined by the UA2 Technique .... 135

63 The Distributions of the Interaction Vertices from 1987 Data. 149

64 The Number of Jets, as Function of Jet $\eta$, with More than $10 \%$ of Energy Coming from the Plug Calorimeters ........ 151

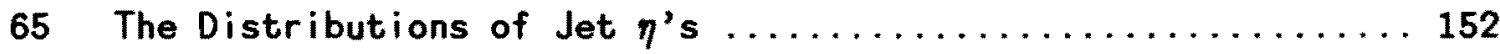

66 Uncorrected Pt Spectra for Jets and Cosmic Rays ......... 153

67 The Fraction of the Jet and Cosmic Ray Energy contained in the EM Calorimeters ............................. 154

68 The Missing Pt in Jet and Cosmic Ray Events ........... 155 
69 The Trigger Effect on the Jet Et Spectrum ............ 156

70 The Trigger Effect on the Dijet Mass Spectrum ........... 156

71 Unsmearing Ratios for the Jet Et Spectrum ............ 157

72 Unsmearing Ratios for the Dijet Mass Spectrum ... . . . . . 157

73 Uncertainties in the Jet Et Spectrum $\ldots \ldots \ldots \ldots \ldots \ldots \ldots \ldots$

74 Uncertainties in the Dijet Mass Spectrum ............... 159

75 Parton Pt Spectra according to QCD with Different

Choices of Momentum Scales and Structure Functions ......... 169

76 Diparton Mass Spectra according to QCD with Different

Choices of Momentum Scales and Structure Functions .......... 170

77 Inclusive Jet Et Spectra (CDF, UA1, UA2 and QCD) $\ldots \ldots \ldots \ldots 171$

78 Inclusive Jet $\mathrm{Pt}$ Spectra (CDF, UA1, UA2 and $Q C D) \ldots \ldots \ldots \ldots$

79 Dijet Mass Spectra (CDF, UA2 and $Q C D) \ldots \ldots \ldots \ldots \ldots \ldots$

80 Unsmeared Jet Et Spectrum Compared with QCD with 4 Sets of Structure Functions ............................. 174

81 Unsmeared Dijet Mass Spectrum Compared with QCD with 4 Sets of Structure Functions ................ 175

82 Unsmeared Dijet Mass Spectrum Compared with Calculations Involving the Effects of Axigluons .......... 176

83 Smeared Dijet Mass Spectrum Compared with Calculations Involving the Effects of Axigluons Which Have 5 Decay Channels .................................. 177

84 Smeared Dijet Mass Spectrum Compared with Calculations Involving the Effects of Axigluons Which Have 10 Decay Channels 
85 Smeared Dijet Mass Spectrum Compared with Calculations Involving the Effects of Axigluons Which Have 20 Decay Channels ................................. 179

86 Smeared Jet Et Spectrum Compared with Calculations Involving the Effects of Axigluons Which Have 10 Decay Channels ....... 180

87 The Smeared Jet Et Spectra with Different Compositeness Scales ......................... 181

88 The Smeared Jet Et Spectra with Compositeness with Different Structure Functions ................... 181

89 Comparison of the Smeared and Unsmeared Jet Et Spectra with Compositeness ................................. 182

90 Comparing Compositeness Scale $\Lambda_{C}=700 \mathrm{GeV}$ with Data for a High Pt Bin (to $\infty$ ) When the Low Edge of the Bin Is Varied 183

91 Same as Fig. 90 but QCD Is Compared $\ldots \ldots \ldots \ldots \ldots \ldots \ldots \ldots$

92 The Cross Section for the Deep Inelastic Scattering Process .. 200

93 Diagrams Illustrating the Meaning of Twist ............ 200

94 Graphs for Computing the Matrix Elements of $D_{V}$ and $D_{F} \ldots \ldots 201$

95 Ladder Diagram Giving Rise to Leading Mass Singularities .... 201

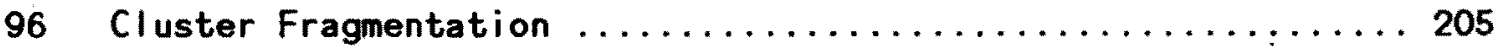

97 The Calorimeter Response as a Function of the Charged Particle Momentum ....................... 214 


\section{INTRODUCTION}

In this thesis we study the jet production cross section in the central rapidity region in pp collisions at a center of mass energy of $1.8 \mathrm{TeV}$. The pseudo-rapidity $\eta$ is defined as $\eta \equiv-\ln (\tan (\theta / 2))$, where $\theta$ is the angle between the directions of the jet momentum and the proton beam. We will call the region $-0.7<\eta<0.7$ the "central rapidity" region by definition. The goal of this thesis is to determine two kinds of differential cross sections for jet production: an inclusive jet Et distribution $\mathrm{d} \sigma / \mathrm{dEt}$, and the distribution in invariant mass $d \sigma / d M_{J J}$ of two jet systems. These spectra serve to check the strong interaction theory, and thus to look for new phenomena, at the highest available energy. The strong interaction theory will be discussed in Ch. 1 and Appendix A, B and C.

The experiment, the Collider Detector at Fermilab (CDF), has been conducted at the Tevatron Collider at Fermi National Accelerator Laboratory, Batavia, IIlinois, USA. In Ch. 2 and 3 we will describe the detector, calorimeters in particular, in some detail. The data we use were collected during the first CDF data run, from February to May 1987. In Ch. 4 we review the performance and problems encountered in that run.

After Ch. 4 we will enter the analysis part of this thesis. First we cite in Ch. 5 several problems that contaminate the data, e.g. cosmic ray background and electronic noise, and the measures to clean them up. We then show in Ch. 6 some characteristics of jets as observed in the CDF detector, and compare them with Monte Carlo data. We found that there were problems remaining in the Monte Carlo simulation. Then in Ch. 7 we use Monte Carlo data to determine the loss of jet energy due to the cracks in the detector, leakage out of the back of the detector and nonlinearity in the response of the detector. Corrections must be applied to the measured jet energy and momenta. After event selection we determine the jet spectra in Ch. 8. In the last chapter, $\mathrm{Ch}$. 9, we compare the measured spectra with QCD and nonstandard theories such as the compositeness of quarks, and chiral QCD $S U_{L}(3) \times S U_{R}(3)$ which breaks down to ordinary $S U(3) Q C D$ at some energy scale leaving an octet of massive "axigluons". We find QCD is in good agreement 
with the data, though the comparison is not precise enough to tell which parameterization of the structure functions is the best, or what interaction scale (the $Q^{2}$ in $a_{s}\left(Q^{2}\right)$ and structure function $f\left(x, Q^{2}\right)$ ) to choose. We have set a limit on the quark compositeness scale $\Lambda_{c}>680 \mathrm{GeV}$ (compared to $400(370) \mathrm{GeV}$ set by UA1(UA2)) at $95 \%$ confidence level. Assuming an axigluon mass width $\Gamma_{A}=N a_{S} M_{A} / 6$, where $N$ is the number of decay channels and $M_{A}$ is the axigluon mass, we have excluded at the same confidence level the axigluon masses:

$$
\begin{aligned}
& N=5,120<M_{A}<210 \mathrm{GeV} \\
& N=10,120<M_{A}<150 \mathrm{GeV}
\end{aligned}
$$

We are not able to rule out axigluons of any mass with $N=20$. This is due to the low statistics in the high dijet mass region and the unsuitability of the Tevatron energy for probing the low mass region. 


\section{CHAPTER 1}

\section{JET PRODUCTION IN HADRON COLLISIONS}

In this thesis we study "jet" production in the collision of protons and antiprotons at a center of mass energy of $1800 \mathrm{GeV}$. This chapter provides a theoretical understanding of the process. A "jet" is a group of particles which are produced in the collision and are closely related in the directions of their momenta. In fact, according to the current theory, each jet is a manifestation of a scattered sub-nuclear particle which we will call a parton. The partons make up all hadrons (particles interacting with the strong force, including protons, neutrons, pions...), but cannot be separated as individual free particles due to the strong binding between them. As a consequence of the strong binding, an energetic parton that is trying to break away from the rest of the system must trade its energy by pulling out from the vacuum more partons which then condense into a group of hadrons -- a jet.

There are two classes of partons: the building blocks of matter, called quarks (spin-half), and the force-mediating particles (spin-1), called gluons. A proton, for example, is built from three quarks which are bound together by "virtual" gluons (strong force). But when we see it with a very powerful "microscope" -- a very energetic particle beam -- a different picture will appear, because the quarks are in fact continuously emitting and reabsorbing gluons and the gluons can in turn become quark pairs. Thus a hadron is really a cloud of quarks and gluons. Furthermore the distribution of partons is energy dependent (the wavelength used in the microscope) as more and more virtual emissions can be seen as we probe deeper. When two hadrons collide, a pair of partons may be selected at random, one from each hadron according to the parton distributions, to make a very hard collision. The scattered partons then make their ways into hadrons which will be detected by the detectors. But most of the time no such hard collision occurs (no jets), and the event is called a "minimum bias" event. 
This chapter is organized as following. First we review the story of the birth of a very successful theory "QCD" (Sect. 1.1) and its encouraging result (Sect. 1.2) that the strong force becomes weaker and weaker as one goes deeper and deeper into the hadrons. We then discuss the factorization of the jet production cross section into parton distributions and the elementary cross sections of the parton collisions (Sect. 1.3). In Sect. 1.4 we briefly discuss the theoretical prediction of the energy dependence ("evolution") of the parton distributions. This section is substantiated by the materials in Appendix A, where three ways of deriving the evolution are discussed and some of the parameterizations of parton distributions are reviewed in detail. We then talk about the methods of making partons into hadrons ("hadronization", Sect. 1.5 and Appendix B), and the treatment of the remnants of the beam particles after the collision which must also be made into hadrons (Sect. 1.6 and Appendix $\mathrm{C}$ ). A correct treatment of the initial and final state radiation, the hadronization, and the remnants is important for our Monte Carlo study of correcting the measured jet energy for cracks, leakage and nonlinear response of the detector (in Chapter 7). Finally we consider models with non-standard features: composite quarks (Sect. 1.7.1) and "chiral" QCD (Sect. 1.7.2).

\subsection{The Story of Quantum Chromodynamics (QCD)}

Gell-Mann and Ne'eman [1] proposed an SU(3) symmetry as an extension of the isospin symmetry (Heisenberg 1932) of baryons and mesons to include strange particles in 1961. The model was a success and in 1964 Gell-Mann and Zweig [2] interpreted the symmetry in terms of quarks, $u^{2 / 3}, \mathrm{~d}^{-1 / 3}$, and $s^{-1 / 3}$ with the fractional charges given by the Gell-Mann-Nishijima relation: $Q=B / 2+S / 2+I_{3}$. But this simple quark model did not allow consistent Fermi-Dirac statistics, and in 1965 Han and Nambu [3] proposed three triplets of quarks of integral charges to carry the extra internal quantum numbers needed for Fermi statistics. Further theoretical considerations and experiments [4] have favored the ideas of fractional charges and three types of quarks (red, green, and blue) for each flavor $(u, d, s \ldots)$. 
Sometime in 1969 when Feymman was considering a relativistic hadron as a beam of partons, each with a fraction $X_{F}$ of the hadron momentum, to explain the particle production which seemed to indicate a soft constituent exchange between hadrons, Bjorken [5] derived his scaling hypothesis assuming free field behavior of the current commutator $\left[J_{\mu}(x), J_{\nu}(0)\right]$ near the light cone $\left(x^{2} \rightarrow 0\right)$. When experiments at SLAC confirmed Bjorken's scaling, it took the puzzled Feynman only one evening to figure out that the Bjorken's scaling parameter $-q^{2} / 2 q . p$ was in fact equivalent to $h$ is $X_{F}$ in the scaling limit and the scaling function was the probability of finding a parton with momentum $x_{F} p$ weighted by the square of the electric charge [6]. The scaling behavior told that the partons are essentially free particles inside hadrons, which contradicted the naive notion that quarks were strongly bound.

Thus the discovery of Bjorken's scaling led to a powerful criterion for any viable strong interaction theory: it had to be asymptotically free. Another clue was provided by the empirical fact that no free quark was ever found, thus the theory ought to have a phase of confinement. Originally the quark- binding force was taken to be the color singlet vector field. In the years of 1972 and 1973 Fritzsch, Gell-Mann and Leutwyler [7] argued the advantages (including the above mentioned clues and the absence of a long range force, etc.) of a gluon octet (adjoint representation of SU(3)) over the singlet gluon, and Politzer, Gross and Wilczek [8] showed that nonabelian gauge theories are asymptotically free. Coleman and Gross [9] further proved that no theory without non-abelian gauge bosons could be asymptotically free. The choice of a SU(3) gauge theory for the strong interaction, called Quantum Chromodynamics (QCD, a name given by Gell-Mann) was then clear.

\subsection{An Asymptotically Free Theory}

Although the renormalizability of the Yang-Mill gauge theory (1954) was conjectured in the early days by power counting, it was to be proven by $t$ 'Hooft [10] in 1971. The theorem was shown to hold even when the symmetry is broken by the Higgs mechanism. The renormalizable SU(3) gauge theory for 
the strong interaction (QCD) assumes three types of quarks $\left(\phi_{r}, \phi_{g}, \phi_{b}\right)$ i for each flavor $\left(\psi_{i}=u, d, s, c \ldots\right)$ and an octet of color field $A_{a}$, and the Lagrangian reads

$$
L=\frac{1}{4} F_{a}^{\mu \nu} F_{a \mu \nu}+i \bar{\phi}_{j} \gamma_{\mu} D_{j k}^{\mu} \phi_{k}-\bar{\gamma}_{j} M_{j k} \phi_{k},
$$

where

$$
\begin{aligned}
& F_{a}^{\mu \nu}=\partial^{\mu} A_{a}^{\nu}-\partial^{\nu} A_{a}^{\mu}+g f_{a b c} A_{b}^{\mu} A_{c}^{\nu} \\
& D_{j k}^{\mu} \equiv \delta_{j k} \partial^{\mu}-i g\left(T_{a}\right)_{j k} A_{a}^{\mu}
\end{aligned}
$$

with $\left[T_{a}, T_{b}\right]=i f_{a b c} T_{c}$ being the SU(3) algebra. The effective coupling $\bar{g}^{2}\left(Q^{2}\right)$ can be shown [8] by the technique of renormalization group (RG) [11] to be (see Appendix A for the derivation)

$$
\bar{g}(t)^{2}=\frac{g^{2}}{1+b g^{2} \ln \left(Q^{2} / \mu^{2}\right)} \equiv \frac{1}{b \ln \left(Q^{2} / A^{2}\right)}
$$

where

$$
b \equiv \frac{1}{16 \pi^{2}}\left[\frac{33}{3}-\frac{2}{3} N_{f}\right], \quad N_{f} \equiv \text { number of flavors }
$$

and $\mu$ is some convenient renormalization point. Eq. (1.2) is exactly the sum of all leading logarithms to all orders (terms like $\left(a \ln \left|q^{2}\right|\right)^{n}$, but not $a^{m}\left(a \ln \left|q^{2}\right|\right)^{n}$ with $m>0$ ) in perturbation theory. It implies $g^{2}(t) \rightarrow 0$ for $t \rightarrow \infty$ if $N_{f}<16$.

With the welcome asymptotic freedom, that the running (evolving with momentum scale $Q^{2}$ ) coupling becomes smaller as the scattering gets deeper, it became possible to work in the framework of renormalizable perturbation theory. Remember this was not the case in the old meson field theory where the coupling constant was intractably large, leaving the 50s' and 60s' theoretical physics under the reign of the "S-matrix". The new light is that strong interaction theory can be calculated and tested experimentally at least for very high Pt processes. But the calculations are still haunted by several uncertainties (which unfortunately will be matched by also large experimental uncertainties) which we will see in the following sections. It turns out that no strong theory in any form has ever been simple. The 
formidable mathematical complexity, to which the strong theory is married, has never eased.

\subsection{The Process of Hadron Collisions}

Thus the asymptotically free QCD theory endorses (or is endorsed by) the picture of quasi-free partons inside hadrons. But the naive Bjorken scaling is only approached logarithmically. In the QCD improved parton picture, virtual partons can be emitted and reabsorbed in a time allowed by the uncertainty principle. This short period of time is Lorentz dilated in the "infinite momentum frame" and the virtual emissions become quasi-real. In a large $Q^{2}$ hadron collision, the two-hadron system can be seen as two essentially decoupled parts: the partons that make hard collisions and the rest of the constituents of hadrons ("spectators"). Correspondingly the cross section can be factored into two parts: the distribution of partons inside hadrons and the cross section of the parton collision. The cold part (spectators, or "beam jets") will be discussed in Sect. 1.6 and Appendix C. The parton distributions, which can be predicted by QCD based on low energy measurements, will be discussed in the next section (1.4) and appendix A. The elementary parton cross sections will be given here. For high $Q^{2}$, where $a\left(Q^{2}\right)$ is small, they have been calculated to the lowest order by several authors [12]:

$$
\begin{aligned}
& \sigma\left(q_{i} q_{j}^{2} \rightarrow q_{i} q_{j}^{2}\right)=\frac{4 a^{2}}{9 s} \frac{s^{2}+u^{2}}{t^{2}} \\
& \sigma\left(q_{i} \bar{q}_{i} \rightarrow q_{j} \bar{q}_{j}\right)=\frac{4 a^{2}}{9 s} \frac{t^{2}+u^{2}}{s^{2}}, i \neq j \\
& \sigma\left(q_{i} \bar{q}_{i} \rightarrow q_{i} \bar{q}_{i}\right)=\frac{4 a^{2}}{9 s}\left[\frac{t^{2}+u^{2}}{s^{2}}+\frac{s^{2}+u^{2}}{t^{2}}-\frac{2 u^{2}}{3 s t}\right] \\
& \sigma\left(q_{i} \bar{q}_{i} \rightarrow g g\right)=\frac{8 a^{2}\left(t^{2}+u^{2}\right)}{3 s}\left[\frac{4}{9 t u}-\frac{1}{s^{2}}\right] \\
& \sigma\left(g g \rightarrow q_{i} \bar{q}_{i}\right)=\frac{3 a^{2}\left(t^{2}+u^{2}\right)}{8 s}\left[\frac{4}{9 t u}-\frac{1}{s^{2}}\right]
\end{aligned}
$$




$$
\begin{aligned}
& \sigma(g q \rightarrow g q)=\frac{a^{2}\left(s^{2}+u^{2}\right)}{s}\left[\frac{1}{t^{2}}-\frac{4}{9 s u}\right] \\
& \sigma(g g \rightarrow g g)=\frac{9 a^{2}}{2 s}\left[3-\frac{t u}{s^{2}}-\frac{s u}{t^{2}}-\frac{s t}{u^{2}}\right]
\end{aligned}
$$

where $s, t$ and $u$ are Mandelstam variables for "partons". And the "two-jet" cross section in the hadron collision $(a+b \rightarrow 1+2)$ is given by

$$
\frac{d \sigma}{d y_{1} d y_{2} d p_{t}}=\frac{2 \pi \tau}{s} \sum_{i j} f_{i}^{a}\left(x_{a}, Q^{2}\right) f_{j}^{b}\left(x_{b}, Q^{2}\right) \sigma_{i j}(s, t, u)
$$

where $\tau \equiv s_{\text {two-parton }} / s_{\text {two-hadron. The inclusive jet cross section is }}$ obtained by integrating the above equation over $y_{2}$. The invariant mass distribution of two jets, both in the rapidity region $[-Y, Y]$, is obtained by integrating $y_{1}$ and $y_{2}$ as

$$
\frac{d \sigma}{d M}=\frac{\pi \tau M}{4} \int_{-Y}^{Y} d y_{1} \int_{Y \min }^{Y_{\max }} d y_{2} \sum_{i j} \frac{1}{s \cosh ^{2} y^{*}} f_{i}^{a}\left(x_{a}, Q^{2}\right) f_{j}^{b}\left(x_{b}, Q^{2}\right) \sigma_{i j}(s, t, u)
$$

where

$$
\begin{aligned}
& y^{*} \equiv \frac{1}{2}\left(y_{1}-y_{2}\right), \\
& Y_{\min } \equiv \max \left(-Y, \ln \tau-y_{1}\right), \\
& Y_{\max } \equiv \min \left(Y,-\ln \tau-y_{1}\right) .
\end{aligned}
$$

\subsection{QCD Evolution of Structure Functions}

In principle, the $Q^{2}$ dependent structure functions should be totally predicted by the content of the QCD Lagrangian. But since the nonperturbative aspect of QCD is far from being completely solved, it is necessary to factor out the incalculable part which must be determined by experiment. For very low $a^{2}$ it is not meaningful to talk about partons. Thus we need a sufficiently large $Q_{0}^{2}$, where perturbation theory works reasonably well, as the experimentally determined starting point for the $Q^{2}$ evolution which is predicted by perturbative QCD. Only when the perturbative aspect of $Q C D$ (the $Q^{2}$ evolution of coupling and structure 
functions, and the cross sections as the results of the assumed fieldmatter couplings) is vindicated, can one have the right to hope that it is a correct theory in general.

In Appendix $A$ we review three different ways of deriving the $Q^{2}-$ evolution of the structure functions: by a formal field approach, by integrating the famous Altarelli-Parisi equations, and by summing up the Bethe-Salpeter ladders (the diagrams of leading logarithms). As in the case of Bjorken scaling, the QCD evolution was first derived from field theory, which then inspired (and justified) the "visibly" superior A-P equations. The basic idea behind the A-P approach is very intuitive. By increasing the resolving power of the "microscope" from $Q^{2}$ to $Q^{2}+\Delta Q^{2}$, one can discern a virtually emitted parton (from another parton) which could not be resolved at $Q^{2}$. Define $t \equiv \ln \left(Q^{2}\right)$, there is a chance $d t \cdot(a(t) / 2 \pi) \cdot P(z) d z$ that a quark carrying a fraction $y$ of the proton momentum radiates a gluon and reduces its energy from $y$ to $x=y z$. The parton density at $x$ simply receives all contributions from $y>x$. The decay function $P(z)$ can be derived from the QCD vertices, but the function $a(t)$ must be determined elsewhere (field theory, Sect. 1.2).

The results in Appendix $A$ can be summarized as following. The moments, $M_{n} \equiv \int_{0}^{1} d x x^{n-2} M(x)$ (see eq. (A21)), of the flavor non-singlet (or the diagonalized) structure functions (eq. (A39)-(A41)) have the form

$$
M_{n}\left(Q^{2}\right)=M_{n}\left(Q_{0}^{2}\right)\left[a\left(Q_{0}^{2}\right) / \alpha\left(Q^{2}\right)\right]^{-a}
$$

where

$$
\alpha^{2}\left(Q^{2}\right) \equiv g^{2}(Q) / 4 \pi, \quad a \equiv \gamma / 8 \pi a b
$$

with b being defined in eq. (1.3) and $\gamma$ the anomalous dimension of the corresponding operator (see eq. (A8), (A35) and (A36)). These equations for the moments are the leading log (to all orders) predictions in closed form. But this moment analysis has a drawback because it is very hard to measure the structure functions at very small and large $x$. With the aid of today's computers, it is possible to work directly in the $x$ space with the Altarelli-Parisi integro-differential equations (eq. A49, A53, and A54 in Appendix A) . 
There have been several sets of parameterizations of the structure functions proposed by several authors, all starting from low energy data and mostly parameterizing the evolved structure functions in terms of $Q^{2}$. In Appendix A (Sect. A.3) we review two of the most popular ones (not necessarily the best): Duke-Owens' (DO) and Eichten-Hincliffe-Lane-Quigg's (EHLQ). In Ch. 9 we will use these four sets of parameterizations to do QCD calculation and compare the results with data.

\subsection{Hadronization of Quarks And Gluons}

Since no quarks have ever been found and QCD seems to indicate a phase of confinement, all observable objects are supposed to be color singlets. The transition from partons (quarks and gluons) to hadrons ("jets") is called hadronization or fragmentation. The number of models to do this seem to reflect the degree of vagueness in the understanding of the subject. Three fragmentation schemes are discussed in Appendix B. In the independent fragmentation scheme each parton is evolved independently into hadrons according to a parameterized momentum distribution of hadrons. In the string fragmentation strings (field flux tubes) are stretched between quarks and break into lighter hadrons. And in the cluster fragmentation each high virtuality $\left(\left(p^{2}\right)^{1 / 2}\right)$ parton is evolved into a shower of partons of lower virtuality, from which color-singlet clusters are formed and decay into pairs of light hadrons. In $\mathrm{Ch}$. 7 we will determine by Monte Carlo simulation the necessary corrections to the jet energy for the cracks in the detector, leakage out of the back of the detector and the nonlinearity of calorimeter response (Fig. 97). The fragmentation scheme affects the result because the measured energy does depend on the momentum distribution of the particles inside a jet due to the nonlinearity. In the event generator we use for the study, ISAJET [13], a parton showering stage takes place before the final partons are hadronized by the independent fragmentation. In this mixed scheme the virtuality cutoff for the parton branching need to be set higher (6 GeV in ISAJET, as compared to 1-2 GeV in other Monte Carlo's) to insure that the number of final hadrons will not be too high. 


\subsection{Beam Jets and Initial State Radiation}

The remaining cold part of each baryon, after being stripped off one parton, must also suffer softer impacts and find its way into final hadrons. We discuss the beam jets and initial state radiation in some detail in Appendix $C$. They also affect our jet energy correction as they are the ever-present background in any kind of $\bar{p} p$ events. In ISAJET the initial state radiation is generated by a backward evolution from the initial state partons that make the hard collision, and the beam jets are treated as a variable number of "cut Pomerons", each of which corresponds to a pair of back-to-back low Pt jets. The initial state radiation and Pomerons are hadronized by the independent fragmentation scheme.

\subsection{Beyond QCD}

\subsubsection{Are Quarks Composite? [12]}

The multiplicity of quarks and leptons has lead one to speculate that they might be composite as well. As a natural extension of the technicolor model that constructs the Higgs sector from the so-called techniquarks, the quarks can also be assumed to be composed of more fundamental particles often called "preons". Preons are supposedly bound together by a new kind of asymptotically free and infrared confining gauge force ("metacolor"). It has been pointed out by 't Hooft that under certain conditions, confining theories which possess global chiral symmetries may lead to the existence of massless composite fermions when the chiral symmetries are not spontaneously broken. The observed masses of quarks and leptons may be accounted for by a small bare mass for the preons, or a preon weak interaction that explicitly breaks the chiral symmetries. It is conceivable that different fermion species might have different compositeness scales.

At subenergies of a few times the compositeness scale $\Lambda^{*}$, multiple production processes, which will be geometrical in magnitude (of order $4 \pi / \Lambda^{* 2}$ ), would dominate over the familiar two body parton scattering processes (decreasing inversely with $s$, of order $\pi a^{2} / s$ ). Any substructure 
of quarks and leptons will modify the gauge field propagator by a form factor

$$
F\left(Q^{2}\right)=1+Q^{2} / \Lambda^{* 2} \text {, }
$$

where $Q$ is the four momentum carried by the gauge field. But if $\Lambda^{*}$ is large compared to the available subenergy, the composite interaction can be approximated by the 4-fermion contact interaction of the form (the low energy manifestation of constituent exchanges) [14]:

$$
L_{f f}=\left(g^{2} / 2 \Lambda^{* 2}\right)\left(\eta_{L L} f_{L} \gamma^{\mu} f_{L} f_{L} \gamma_{\mu} f_{L}+2 \eta_{L R} f_{L} \gamma^{\mu} f_{L} f_{R} \gamma_{\mu} f_{R}+\eta_{R R} f_{R} \gamma^{\mu} f_{R} f_{R} \gamma_{\mu} f_{R}\right) \text {, }
$$

where $g$ is the strong coupling constant, $f_{L, R}$ are the left and right components of the fermion field, $\gamma_{\mu}$ the dirac matrices, and $\Lambda^{*}$ is defined such that the largest $\left|\eta_{i j}\right|=1$. In the region $s<\Lambda^{* 2}$, the effect of contact terms is far larger than that expected from a form factor.

In chapter 9, we will show numerically the effect of these contact terms on the inclusive jet Pt spectrum (Fig. 87). The effect is very striking in the high Pt region where the spectrum flattens because the valence quark interactions become increasingly dominant and the cross section approaches a geometrical limit.

\subsubsection{Is QCD Chiral?}

Although SU(3) QCD is consistent with all existing data, there is a possibility that $S U(3)$ is really a subgroup of a larger symmetry group of the strong force. P. Frampton and S. Glashow [15] proposed that high energy QCD might be chiral $S U(3)_{L} \times S U(3)_{R}$, but broken down to ordinary $S U(3)_{V}$ at some scale, probably (not necessarily) comparable to the Fermi mass $M-250$ GeV where the electroweak interaction is broken. But in order to make the theory free of triangle anomalies (for renormalizability) with respect to the larger gauge group, additional fermions must be added. Moreover a rich system of scalar mesons is needed to break the symmetry. These particles enrich the desert which was a threatening feature of the standard model.

There are many ways of implementing the representations of the group. In ref. 15 five types of models, either with divers quark assignments or with the introduction of exotic" color quantum numbers for cancellation of anomalies, were considered. They vary in the particle contents, but have 
things in common: the necessary existence of more than three families of quarks and leptons, a massive octet of vector bosons (the axigluons), and a rich spectrum of scalar mesons for symmetry breaking. Later Frampton and Glashow [16] made their preferred choice based on additional "prejudices", among them the GIM mechanism. The most promising model involves five standard families of quarks and leptons, and two species of exotic colored fermions: a quix $[(\overline{6}, 1)+(1,6)]$ and a dichromatic multiplet $[\overline{3}, 3]$.

The Feynman vertices involving axigluons are shown in Fig. 1 [17]. Because the strong interaction is assumed to be parity conserving, no gluon-axigluon vertices with an odd number of axigluons are allowed. The diagrams that give the axigluon contribution to $2 \rightarrow 2$ scattering are shown in Fig. 2. Since the axigluon can be produced strongly and decays into two jets, its existence should show up in the dijet mass spectrum as a bump, which we will look for in the analysis described in this thesis.

For the models with the simplest symmetry breaking, the mass of the axigluon $M_{A}$ is expected to be no more than $300 \mathrm{GeV}$. Cuypers and Frampton [18] determined $M_{A}>9 \mathrm{GeV}$ from upsilon decay. Robinett [19] showed that partial wave unitarity requires $M_{A}>30 \mathrm{GeV}$. Bagger, Schmidt and King [17] $(B-S-K)$ calculated the axigluon contribution to the jet cross section assuming an axigluon mass width $\Gamma_{A}=\mathrm{NaM}_{A} / 6$, where $N$ is the number of axigluon decay channels (i.e. the number of light quarks, up to $18=$ 10 (standard families) + 5(quix) + 3(dichromatic) for the most promising model), and excluded the mass region $125<M_{A}<275 \mathrm{GeV}$ for $N<20$ from the UA1 data. Later UA1 [20] excluded masses between 150 and $310 \mathrm{GeV}$ at a $95 \%$ confidence level for $\Gamma_{A}<0.4 M_{A}$. UA1 added up cross sections incoherently (QCD spectrum + Breit-Wigner resonance), but they noted that once the detector resolution is taken into account there is no noticeable difference between the predicted shape of the axigluon modified dijet mass spectrum and their simple model. So the regions not excluded by experiments so far seem to be $30<M_{A}<125 \mathrm{GeV}$ and $M_{A}>310 \mathrm{GeV}$. But unless something is very unusual, the simplest models do not suggest $M_{A}>310 \mathrm{GeV}$.

We note from Fig. 2 that in a dijet mass distribution a mass bump near the axigluon mass $m_{A}$ is due to $q q$ or $q \bar{q}$ scattering (no axigluon in the final state), because any final state with an axigluon (and consisting of 
two detectable jets) must have a dijet mass much larger than $m_{A}$. In very high energy collisions, the detection of a low mass axigluon suffers two things: a) the dominant process in this region is gluon scattering and thus the bump is less clear, and b) the parameterization of the gluon distribution has a larger uncertainty, making the prediction less certain. We will see in $\mathrm{Ch}$. 9 that the mass region where the CDF has the advantage over the lower energy Spps experiments is probably $m_{A}>250 \mathrm{GeV}$. 


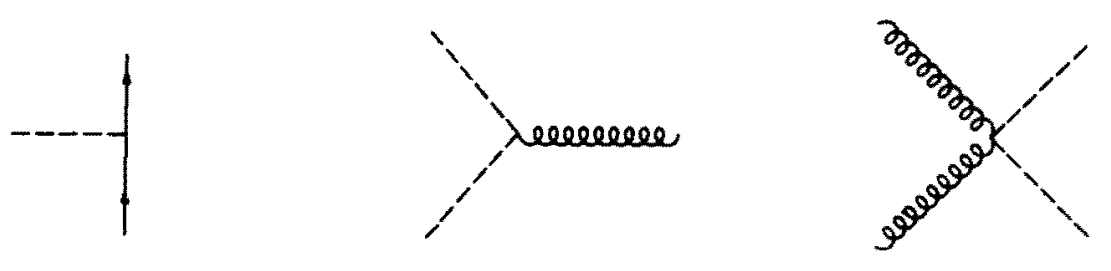

Fig. 1 Feynman vertices involving axigluons. No gluon-axigluon vertices with an odd number of axigluons are allowed if parity is conserved.
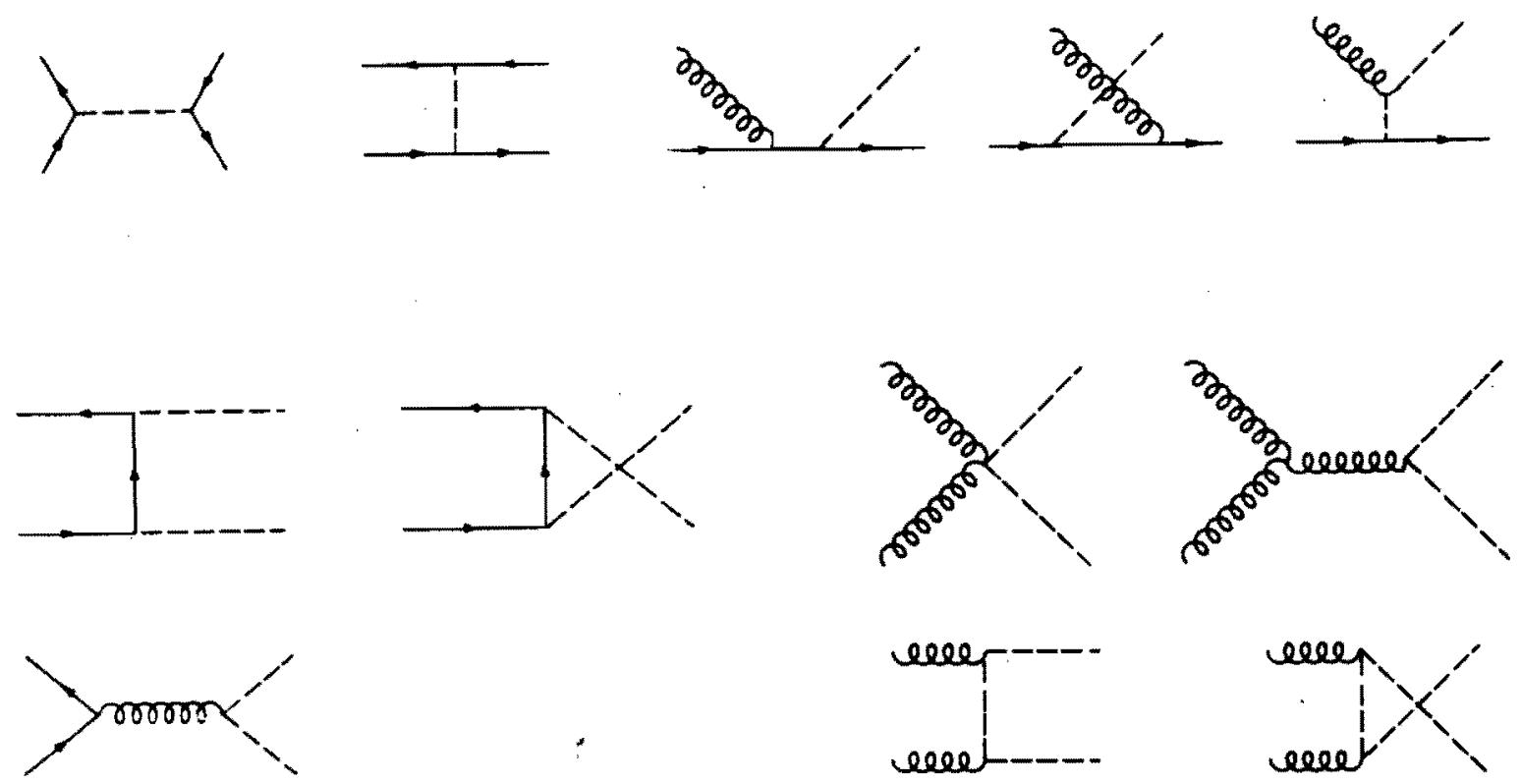

Fig. 2 The axigluon contribution to the lowest order 2 parton to 2 parton scattering. 
CHAPTER 2

\section{THE COLLIDER DETECTOR AT FERMILAB (CDF)}

In this chapter and the next chapter we will describe the instrument we used for jet analysis, the CDF detector. Here we will concentrate on the detector itself, and describe the electronics in the next chapter. The Tevatron is currently. the world's highest energy proton and antiproton collider, with center of mass energy of $1.8 \mathrm{TeV}$. CDF is the first large solid angle detector to take data at it. There has been a series of papers detailing the construction and performance of each component published in Nuclear Instruments and Methods in Physics Research (NIM) in the years of 1987 and 1988. In this chapter and the next chapter are condensed versions (though not exactly) of these papers, put together in one place for easy reference. We have omitted much of the details, but not any of the essential elements of the geometry and working principles. Our emphasis will be on the calorimeters (Sect. 2.2), especially the central calorimeters, which measure the energy of jets. Among the tracking systems, which make accurate momentum measurement of the charged particles but can not see the neutral particles, only one will be mentioned -- the time projection chamber (Sect. 2.3) used to determine the position of each collision.

\subsection{Dverview [21]}

A perspective view of the detector is shown in Fig. 3 and a cut-away view is in Fig. 4. It weighs 5000 tons and consists of three parts: a movable 2000 ton central detector (covering the angular region $10^{\circ}<\theta<$ $170^{\circ}$, where $\theta$ is the polar angle relative to the proton beam direction) and the two identical forward $\left(\theta<10^{\circ}\right)$ and backward $\left(\theta>170^{\circ}\right)$ detectors.

The central detector consists of the Vertex TPC (VTPC), Central Tracking Chamber (CTC), superconducting magnet coil, Central EM Shower Counter (CEM), Central Hadron Calorimeter (CHA), Central Muon Chamber (CMU), End 
Wall Hadron Calorimeter (WHA), Plug EM Shower Counter (PEM), PIug Hadron Calorimeter (PHA) and Forward (small angle) Tracking Chamber (FTC). PEM and PHA make up the cone-shaped end plug detectors $\left(10^{\circ}<\theta<30^{\circ}\right.$ and $150^{\circ}<\theta$ $\left(170^{\circ}\right)$. The CEM and $\mathrm{CHA}$ calorimeters are contained in $4815^{\circ}$-wedges which are stacked to form 4 self-supporting arches. Individual arches can be retracted for access to the sides of the wedges. The superconducting coil ( $3 \mathrm{~m}$ diameter, $5 \mathrm{~m}$ long), the wall hadron calorimeter and the end plug calorimeters are supported by a steel yoke which forms a "box" $9.4 \mathrm{~m} \mathrm{high}$ by $7.6 \mathrm{~m}$ wide by $7.3 \mathrm{~m}$ long. The whole yoke assembly, with the arches resting on its base, rolls from the assembly hall to the Tevatron beam I ine (31.4 $\mathrm{m}$ in distance).

The forward/backward detectors are made up of time-of-flight trigger counters (Beam-Beam Counters or BBC), Forward EM Shower Counter (FEM), Forward Hadron Calorimeter (FHA), and the steel toroidal Forward Muon Spectrometer (FMU). Particles produced at angles less than $10^{\circ}$ to either beam exit the conical hole in the end plug detector and strike the trigger counter and the calorimeters behind. These detectors are not movable and are permanently garrisoned inside the collision hall (Fig. 5).

\subsection{The Calorimeters}

The calorimeters measure the energy of particles coming out from the collisions. Although the calorimeter, resolution is poorer than that of tracking systems, the calorimeters can "see" the energy of neutral particles which trackers can not. The way the CDF calorimeters see particles is the following. When a particle goes through a dense material (the "absorber", steel or lead), a cascade of particles including a bunch of charged particles will be developed ("showering") due to multiple scattering of the primary (the original), secondary, tertiary ... particles. The charged particles are then sampled by either the ionization energy or the scintillation light they produce in some active medium (gas or scintillator). There are two kinds of cascades, a hadronic one due to the nuclear interaction and an electromagnetic (EM) one. An electron or photon will develop EM shower and the best absorbers for them are those 
with high atomic numbers because there are more electrons in the material. Nucleons and charged pions and other hadrons will start a hadronic cascade, but also develop an E.M. component. The best materials for hadron cascades are those with high number of nucleons. Generally more material is needed for a hadronic shower. Hence the logical arrangement of different types of calorimeters is to put the hadronic one behind the EM one, thus electrons and photons will be contained completely in the EM compartment, leaving only hadrons to the hadron compartment. In CDF the absorbers are lead for the EM calorimeters and steel (cheap and convenient for construction) for the hadron calorimeters. There are also two types of sampling mediums: scintillator and gas. Scintillator has better resolution and is very reliable, but is not suitable for the small angle region where the segmentation must be small.

In this thesis we will study jets in directions almost perpendicular to the colliding beams, i.e. with "pseudo-rapidity" $\eta \equiv-\ln (\tan (\theta / 2)$ not too far away from zero; thus the central scintillator calorimeters are our primary instruments, whose construction and calibration we will describe in some detail. But the forward gas systems also play a part in the topology of an event, and for completeness they will not be omitted.

The CDF $4 \pi^{n}$ calorimeter covers the full azimuth $(\phi)$ and the polar angles $1.7^{\circ}<\theta<178.3^{\circ}$. The space of $\phi$ and $\eta$, which is a very useful representation of phase space for studying hadronic collisions (Appendix D), is divided into rectangular cells as shown in Fig. 6. The cells are 0.1 units in $\eta$ and $15^{\circ}$ (in the central region) or $5^{\circ}$ (in plug and forward regions) in $\phi$. A cell is a "tower" (stack) of interleaved absorber and sampler layers. The towers are projective, i.e. they point at the nominal collision point (Fig. 7), and each has an EM shower counter in front of a hadron calorimeter as in Fig. 4. The physical tower size ranges from about $24.1(\eta) \times 46.2(\phi) \mathrm{cm}^{2}$ in the central region to $1.8 \times 1.8 \mathrm{~cm}^{2}$ in the forward region. In construction, the calorimeters are made of steel (hadron) or lead (EM) plates alternated with plastic scintillators (in the central region) or gas proportional chambers (in the plug and forward regions). A summary of the properties of all calorimeters is given in Tables 1 and 2 . 


\subsubsection{The Central EM Calorimeters (CEM) [22]}

The barrel-shaped central calorimeter starts at a perpendicular radius of $68 \mathrm{in}$. from the beam line. The calorimeter is made up of $4815^{\circ}$-wedges. In the CEM compartment of each wedge there are thirty-one layers of $5 \mathrm{~mm}$ thick SCSN-38 polystyrene scintillator interleaved with 30 layers of $1 / 8$ in. lead; clad on both sides with 0.015 in. aluminum (total 18 radiation lengths and 1 absorption length). The layers are assembled to form 10 projective towers, each subtending 0.11 units of $\eta$ and $15^{\circ}$ in $\phi$. In order to maintain a constant radiation length as the polar angle varies, acrylic is substituted for lead in certain tower layers.

The blue light from the scintillator is collected by wavelength shifters, $3 \mathrm{~mm}$ thick Y7 UVA acrylic, which fit into the $0.25 \pm 0.02 \mathrm{in}$. gaps between the stack and the steel cover plates (3/16 in. thick) on the $\phi$ sides of a wedge (Fig. 8). The gaps and skins represent $4.8 \%$ of the azimuth. The wavelength shifters are laser cut with notches to form 1 in. wide fingers which are bent, gathered, and glued to UVA acrylic rod lightguides which pass through the hadron calorimeter. Finally the green waveshifted light is viewed by Hamamatsu R580 ( $1.5 \mathrm{in}$. diameter bialkali 10-stage photomultiplier tubes) which draw about $300 \mu \mathrm{A}$ each at approximately $1000 \mathrm{~V}$ giving a gain of about $10^{5}$. The photomultiplier readout saturates at about $350 \mathrm{GeV}$.

To achieve high spatial uniformity of response, the thickness of shifter material has been controlled in order to have uniform attenuation length, and great effort went into developing the laser cutting technique to give uniform optical surfaces. The nonuniformity was reduced to no more than $3 \%$ by a combination of two techniques, a) sanding and painting black the edge of the shifter furthest from the collection fingers to eliminate internal reflection, b) using a backing of controlled reflectivity to eliminate the remaining $25 \%$ variations. The backing utilized the fact that $25 \%$ of the scintillator light passed through the $3 \mathrm{~mm}$ shifter material and could be selectively absorbed or reflected back into the shifter to produce more green light. The response maps for 15 wedges were averaged and black ink patterns were silk screened onto reflective Alzak aluminum. 
A proportional strip chamber is inserted inside each stack between the eighth lead layer and the ninth scintillator layer (about 6 radiation lengths). It determines shower position and transverse development at shower maximum by measuring charge deposition on orthogonal strips and wires for electron identification; we do not use it in this jet analysis.

\subsubsection{The Central and End Wall Hadron Calorimeters (CHA and WHA) [23]}

The relation between CEM, CHA, WHA and Plug calorimeter towers is best shown by Fig. 7. The central and endwall calorimeters combined, both using interleaved layers of steel as absorber and acrylic scintillators as the active medium, cover the polar angles $30^{\circ}<\theta<150^{\circ}$. There are 24 projective towers (each covering approximately 0.11 units of pseudo-rapidity) in each $15^{\circ}$ azimuthal slice, of which 12 are totally in the two central wedges, 6 in the two endwall modules and 6 are shared $\left(40^{\circ}<\theta<52^{\circ}\right.$ and $\left.128^{\circ}<\theta<140^{\circ}\right)$. The central hadron modules with $2.5 \mathrm{~cm}$ sampling are 32 layers deep (4.7 $\Lambda_{\text {abs }}$ ) and weigh about $12000 \mathrm{~kg}$ each. They are contained in $4815^{\circ}$ wedges which are stacked into four free standing "C"-shaped arches. The individual arch can be rolled into and out of the detector. The endwall modules with 5 $\mathrm{cm}$ sampling are 15 layers deep $\left(4.5 \Lambda_{\text {abs }}\right)$ and weigh about $7000 \mathrm{~kg}$ each. They plug into cavities in the CDF magnet yoke and serve as part of the flux return path.

The $1 \mathrm{~cm}$ thick scintillators, PMMA doped with $8 \%$ naphthalene, $1 \%$ butylPBD and 0.01\% POPOP, are shaped to give a tower geometry and have the average dimension $1 \times 35 \times 70 \mathrm{~cm}^{3}$. The light is collected by $0.5 \times 1.0 \mathrm{~cm}^{2}$ wave length shifter strips (UVA PMMA) from the $\theta$-sides of the scintillator sheets (Fig. 9). These shifter strips are doped with $30 \mathrm{mg} / 1$ laser dye \#481, which has an emission peak at about $490 \mathrm{~nm}$. They butt up against clear UVA light guides of the same cross section which form two arrays at the azimuthal sides of the tower (Fig. 10). Each array is glued to a transition piece (PMMA doped with dye \#481) which matches the square cross section to the circular photocathode of a photomultiplier tube.

To retain good energy resolution, attention has been paid to the uniformity of response. The nonuniformity on a scintillator sheet was determined with a ${ }^{90} \mathrm{Sr} \beta$ source and it has been reduced from $30 \%$ to $10 \%$ by 
collecting light from the long $\theta$-sides instead of the shorter $\phi$-side (Fig. 9). This nonuniformity is further smoothed out by alternating the wave length shifter readout in successive calorimeter layers. The calorimeters' longitudinal response was made uniform by a) coupling scintillators to light guides selected according to their $\lambda$ (attenuation length), b) inserting filters between the wavelength shifters and light guides. The longitudinal uniformity was determined using a ${ }^{137} \mathrm{Cs}$ point $\gamma$ source which was moved longitudinally along the tower.

\subsubsection{The Calibration of CEM [24]}

On each transition piece (between the light guide and PM tube) there are two mounting holes for two quartz fibers which connect through optical connectors to an LED flasher system. An additional hole is provided on an acrylic prism glued to the transverse edge of each wave shifter (just above the base plate) for a single quartz fiber which is directly bundled to a wedge based xenon flasher system. The photomultiplier gains are monitored by the LED system while the combined response of the wavelength shifter and the PM tube can be checked by the xenon flashers.

On each wedge a tube and pulley system is permanently mounted to move a ${ }^{137}$ Cs $\gamma$ source through all towers at fixed depths. A pair of parallel brass tubes kept $1.5 \mathrm{in}$. apart are positioned between the eighth lead layer and the ninth scintillator layer (near shower maximum) along the calorimeter centerline (in the beam direction). Another pair of tubes run through the hadron calorimeter between the seventh scintillator layer and the seventh steel layer. The ${ }^{137} \mathrm{Cs}$ source, inside a thin stainless steel sheath, enters the EM (or hadron) calorimeter through one of the holes at the $45^{\circ}$ end plate, traverses inside one of the brass tubes, goes around the pulley at the other side of the calorimeter and comes back through the other tube. This source is used to calibrate the calorimeter by the following procedures. Each wedge was exposed to a $50 \mathrm{GeV}$ electron beam and the gain was adjusted to yield $100 \mathrm{pC}$ on each photo-multiplier tube for electrons hitting the tower center. At the same gains, the ${ }^{137} \mathrm{Cs}$ source was moved at constant speed through all towers and the maximum currents induced in each PM tube were recorded for reference in later routine calibrations using 
source alone. The reproducibility of the test beam calibration by tracking with a source calibration has been studied with three wedges. The tube by tube difference in the beam to ${ }^{137} \mathrm{Cs}$ ratio for successive calibration procedures, about 5 weeks apart, is plotted (Fig. 11). The width of the distribution (the reproducibility) was found to be about $\pm 0.4 \%$.

Several electron runs with beam energy ranging from $10 \mathrm{GeV}$ to $100 \mathrm{GeV}$ were conducted to study the energy response and resolution. The resolution for electrons hitting at tower centers is well described by $\sigma(E) / E=$ $13.5 \% / \operatorname{sqrt}(E \sin \theta)$ ) with $E$ in $G e V$.

\subsubsection{The Calibration of Central and Endwall Hadron Calorimeters [23]}

To calibrate phototube gains, a pulsed laser (nitrogen $320 \mathrm{~nm}$ ) is diffused and distributed by optical fibers to the wavelength shifter coupling blocks in front of all photocathodes. A point-like $\beta$ source serves as a backup system in situations where the laser system is not available (e.g. during the test beam calibration). The absolute calibration in the test beam of hadron calorimeters made use of a "skin" source to set the PM tube voltages (gains) without actually exposing the modules to the test beam. The source is called "skin" because it was moved inside tubes which run between the wedge $\phi$-side cover plate ("skin") and the stack along the tower centerline. Initially some wedges were exposed to a $50 \mathrm{GeV}$ pion beam and gains were adjusted to yield a mean of $100 \mathrm{pC}$ on each PM tube for pions hitting the tower center. At the same gain the mean current $I_{c}$ produced in each PM tube by the "skin" source was measured by averaging the induced peak currents over all the layers. The high voltages of the corresponding PM tubes in all the remaining wedges were then adjusted to get the same current $I_{f}$ in response to the skin source. The currents induced by a movable ${ }^{1 \xi_{7}}$ Cs point source (see last section) at fixed depth were also recorded for reference in later routine calibrations using this source alone. It was believed that wedges could be calibrated in this way without being exposed to the test beam. But nonetheless all the remaining wedges were sent to the test beam and the charges collected for $50 \mathrm{GeV}$ pions were recorded. A study using all the test beam calibrated wedges has shown that 
the modules could be calibrated at a level of 3-4\% with the skin source alone.

Based on this result, all the endwall modules except two have been calibrated only by the "skin" source. The two exceptional endwall modules were adjusted to give $100 \mathrm{pC} /$ tube for $50 \mathrm{GeV}$ pions hitting tower centers. At the same voltages the current induced by a ${ }^{137} \mathrm{Cs}$ line source, longitudinally irradiating all layers at same time, was measured for each phototube. The same values of currents were then set in all other modules by adjusting the PM tube high voltages. Immediately after the HV setting, the laser pulse heights and the currents induced by a movable ${ }^{137} \mathrm{Cs}$ point source at fixed tower depth were recorded to provide references for later routine calibrations.

Again the reproducibility of the test beam calibration by source calibration alone has been studied by repeatedly calibrating some modules in the beam over a span of several months, and was found to be about $1 \%$ for central wedges (CHA). The WHA calibrations are good at the 3-4\% level due to the fact that they were calibrated by sources only. The energy resolution was studied with pion beams ranging in energy from $10 \mathrm{GeV}$ to 150 $\mathrm{GeV}$, and the result is shown in Fig. 12 and Fig. 13. The hadron TDC (one for each PM tube to measure time of $\mathrm{flight}$ ) resolution was found to be less than 1.5 ns and the time dependence upon the pion position in a tower was less than 0.5 ns.

\subsubsection{The Plug and Forward Gas Calorimeters: PEM, PHA, FEM, FHA [25]}

All other calorimeters, which cover $|\eta|$ beyond 1.0 , are proportional chamber based systems. Scintillator is not an ideal choice in this region which requires finer size of towers, because any light guide would introduce a substantial dead/hot region. It is also difficult to make a well defined projective tower by scintillators. But since for a given transverse momentum Pt the energy of a particle is amplified by a factor of $1 / \sin \theta$ and the calorimeter energy resolution grows roughly with $\sqrt{E}$, the Et resolution in this region is essentially comparable to that for the central region using the scintillator calorimeter. 
In order to maintain the gap height of the chamber over a large surface area, all gas calorimeters except the Plug Hadron use proportional tube arrays, instead of parallel plate chambers. The tube array also reduces the solid angle for secondary $\delta$-rays which cause fluctuations in the energy deposition. The PHA has parallel plate chambers of $30^{\circ}$-sectors, supported by $I$ beams in the wire direction (azimuthal) at regular intervals. The EM calorimeters consist of layers of proportional tube arrays interleaved with lead plates. In hadronic calorimeters, lead is replaced by steel. The dimensions of absorbers and tubes are given in Table 2, which contains a good amount of information about the four gas calorimeters. Each plane of the tube array was assembled into a quadrant in azimuth in which all tubes are vertical or horizontal (in PEM they alternate layer by layer to reduce the dead zone near the ends of tubes) in a plane perpendicular to the beam axis. This is illustrated in Fig. 14, where only a PEM layer is shown.

Although the working principle is the same, there are two types of construction. The PEM makes use of resistive plastic (loaded with carbon) tubes glued with epoxy to two fiberglass circuit boards (cathode pads and ground, Fig. 14 and Fig. 15), while the others use a aluminum extrusion ( $T$ cross section) and a circuit board (pads) to form the basic cell structure (Fig. 16). The pads were etched out of the copper plating clad on one side of a fiberglass board. They are segmented with boundaries defined by $\eta$ and $\phi$, and the pad sizes are scaled every other layer so that the resultant towers project back to the nominal beam-beam interaction point. Strips in $\theta$ or $\$$ directions can also be made as in the case of PEM where strips are implemented to provide finer steps for layers around the shower maximum. Pads of each tower are ganged into one (PHA and FHA), two (FEM) or three (PEM) depths for independent readout, while each quadrant of anode wire plane is divided into one (PEM and PHA), five (FEM) or six (FHA) sections. Wire section readout can provide a very detailed longitudinal shower development.

All EM calorimeters were calibrated with electrons and hadron calorimeters with pions in the energy range 20-200 GeV. The calorimeters were mounted to allow alignment of the tower geometry with the test beam. The total induced charge is measured by summing the tower of the highest 
energy with surrounding towers that are above pedestal. The best fits to the energy resolutions are:

$$
\begin{array}{rlrl}
\sigma / E & =0.28 / \sqrt{E} & & \text { PEM } \\
& =0.254 / \sqrt{E}+0.47 \% & & \text { FEM } \\
& =2.83 / E+1.13 / \sqrt{E}+8.6 \% & \text { FHA. }
\end{array}
$$

All gas calorimeters use the same argon-ethane $50 \%-50 \%$ mixture with a small percentage of isopropyl alcohol as a quenching agent. But throughout the early test and calibration runs ethyl alcohol, which can deteriorate the epoxy used in chamber construction, was used. The gas gain, which is a sensitive function of temperature, pressure, high voltage, gas composition, wire diameter and wire position, is monitored by gas-monitor tubes which are irradiated by radioactive sources. The monitor tubes have roughly the same construction as a real calorimeter tube and are distributed regularly near the chambers. Both FEM and PEM use ${ }^{55} \mathrm{Fe}$ since the emitted $5.9 \mathrm{keV} X-$ ray converts in the tube wall into electrons which stop mostly within the gas volume. It has been found by test beam study that this monitoring procedure can track the calorimeter gain to within $1 \%$ for PEM and $2 \%$ for FEM.

\subsection{The Vertex Time Projection Chambers (VTPC) [26]}

Only one of the three CDF tracking systems (VTPC, CTC, FTC) is used in our analysis. The VTPC is used to determine the collision point of each event. Thiso is important because the calorimeter itself can not tell at what angle a jet makes an impact. The VTPC also serves as a way to verify the true nature of an event -- a true beam-beam collision, a collision between beam and a residual gas molecule inside the vacuum beam pipe, a large noise in the calorimeter, or just a false trigger. The VTPC is located immediately outside the beam pipe, and tracks charged particles at angles greater than $3.5^{\circ}$ from the beamline.

The system consists of eight double time projection chambers which are mounted end to end along the beam direction. Each octagonal chamber has a central high voltage grid that divides it into two drift regions $(15.25 \mathrm{~cm}$ long) which are filled with argon-ethane 50/50 at 1 atm and applied with an 
electric field $E=320 \mathrm{~V} / \mathrm{cm}$ (resulting $V_{d r i f t}=46 \mu \mathrm{m} / \mathrm{ns}$ ). The electrons drift away from the central grid until they pass through a cathode grid and enter a proportional chamber endcap. As viewed from the drift region (Fig. 17), the octagonal endcap consists of a cathode grid followed by a plane of field shaping wires $(\Delta d=7 \mathrm{~mm})$, a plane of sense wires $(\Delta d=3 \mathrm{~mm})$ and a resistive ink cathode plane $(\Delta d=10 \mathrm{~mm})$. The wire spacing for both field wires and sense wires is $6.34 \mathrm{~mm}$. The field wires are offset by $1 / 2$ wire spacing in $r$ with respect to the sense wires, and the sense wires in adjacent octants (readout separately) are offset radially also by $1 / 2$ wire spacing. Three rows of pads $(4.12 \mathrm{~cm}$ in $r$ by about $1.4 \mathrm{~cm}$ in $\phi$ ) are located behind the resistive cathode, separated by $150 \mu \mathrm{m}$ of epoxy-fiberglass ( $G$ 10). There are 24 sense wires and 24 cathode pads in each octant. The arrival times of the electrons at the sense wires give a picture of the event in the $r-z$ plane. The active area of the chamber extend from about $r$ $=7 \mathrm{~cm}$ to $r=21 \mathrm{~cm}$. A summary of the properties of the VTPC is given in Table 3 .

Adjacent chambers have a relative rotation angle of $\phi=\arctan (0.2)$ about the beam axis. For tracks passing through at least two chambers, this eliminates inefficiencies near octant boundaries and provides $\phi$ information from small angle stereo. 
Table 1 Properties of the Central Calorimeters.

\begin{tabular}{|c|c|c|c|}
\hline & CEM & $\mathrm{CHA}$ & WHA \\
\hline$|\eta|$ coverage & $0-1.1$ & $0-0.9$ & $0.7-1.3$ \\
\hline Tower size $(\Delta \eta \times \Delta \phi)$ & $0.11 \times 15^{0}$ & $0.11 \times 15^{\circ}$ & $0.11 \times 15^{0}$ \\
\hline $\begin{array}{l}\text { Longitudinal samples } \\
\text { in tower }\end{array}$ & 1 & 1 & 1 \\
\hline Active medium & $\begin{array}{l}\text { polystyrene } \\
\text { scintillator }\end{array}$ & $\begin{array}{l}\text { acrylic } \\
\text { scintillator }\end{array}$ & $\begin{array}{l}\text { acrylic } \\
\text { scintillator }\end{array}$ \\
\hline $\begin{array}{l}\text { Scintillator } \\
\text { thickness }[\mathrm{cm}]\end{array}$ & 0.5 & 1.0 & 1.0 \\
\hline Number of layers & 31 & 32 & 15 \\
\hline Absorber & $\mathrm{Pb}$ & $\mathrm{Fe}$ & $\mathrm{Fe}$ \\
\hline Absorber thickness $[\mathrm{cm}]$ & 0.32 & 2.5 & 5.1 \\
\hline $\begin{array}{l}\text { Total depth } \\
\text { Typical phototube } \\
\text { high voltage [V] }\end{array}$ & $\begin{array}{l}18 x_{0}, 1 \Lambda_{\text {abs }} \\
-1100\end{array}$ & $\begin{array}{l}4.7 \Lambda_{\mathrm{abs}} \\
-1500\end{array}$ & $\begin{array}{l}4.5 \Lambda \text { abs } \\
-1100\end{array}$ \\
\hline Typical phototube gain & $1.2 \times 10^{6}$ & $6 \times 10^{5}$ & $10^{6}$ \\
\hline $\begin{array}{l}\text { Typical tower signal } \\
\text { [pC/Gev] }\end{array}$ & -4 & -4 & -4 \\
\hline $\begin{array}{l}\text { Energy resolution } \\
\qquad(\sigma / \mathrm{E}) \text { at } 50 \mathrm{GeV}[\%]\end{array}$ & 2 & 11 & 14 \\
\hline $\begin{array}{l}\text { Typical position } \\
\text { resolution } \\
\text { at } 50 \mathrm{GeV}\left[\mathrm{cm}^{2}\right]\end{array}$ & $0.2 \times 0.2$ & $10 \times 5$ & $10 \times 5$ \\
\hline $\begin{array}{l}\text { Characteristic } \\
\text { width of azimuthal } \\
\text { boundary region }[\mathrm{cm}]\end{array}$ & 3.5 & 4.1 & $\begin{array}{l}3.8,8.9 \\
\text { alternating }\end{array}$ \\
\hline
\end{tabular}


Table 2 Properties of the Plug and Forward Calorimeters.

\begin{tabular}{|c|c|c|c|c|}
\hline & PEM & PHA & FEM & FHA \\
\hline$|\eta|$ coverage & $1.1-2.4$ & $1.3-2.4$ & $2.2-4.2$ & $2.3-4.2$ \\
\hline Tower size $(\Delta \eta \times \Delta \phi)$ & $0.09 \times 5^{0}$ & $0.09 \times 5^{0}$ & $0.1 \times 5^{0}$ & $0.1 \times 5^{0}$ \\
\hline $\begin{array}{l}\text { Longitudinal samples } \\
\text { in tower }\end{array}$ & 3 & 1 & 2 & 1 \\
\hline Active medium & \multicolumn{4}{|c|}{$\begin{array}{l}\text { Proportional tube chambers with } \\
\text { cathode pad readout }\end{array}$} \\
\hline $\begin{array}{l}\text { Proportional tube } \\
\text { size }\left(\mathrm{cm}^{2}\right)\end{array}$ & $0.7 \times 0.7$ & $1.4 \times 0.8$ & $1.0 \times 0.7$ & $1.5 \times 1.0$ \\
\hline Number of layers & 34 & 20 & 30 & 27 \\
\hline Absorber & $\mathrm{Pb}$ & $\mathrm{Fe}$ & $\begin{array}{l}94 \% \mathrm{~Pb} \\
6 \% \mathrm{Sb}\end{array}$ & $\mathrm{Fe}$ \\
\hline Absorber thickness $[\mathrm{cm}]$ & 0.27 & 5.1 & 0.48 & 5.1 \\
\hline $\begin{array}{l}\text { Total depth } \\
\text { Typical wire } \\
\text { high voltage }[\mathrm{V}]\end{array}$ & $\begin{array}{l}20 x_{0} \\
+1700\end{array}$ & $\begin{array}{l}6 \Lambda_{\text {abs }} \\
+2120\end{array}$ & $\begin{array}{l}25.5 x_{0} \\
+1900\end{array}$ & $\begin{array}{l}8.2 \Lambda_{\text {abs }} \\
+2200\end{array}$ \\
\hline Typical wire gain & $2 \times 10^{3}$ & $2 \times 10^{4}$ & $5 \times 10^{3}$ & $10^{4}$ \\
\hline $\begin{array}{l}\text { Typical tower signal } \\
\text { [pC/Gev] }\end{array}$ & +1.25 & +1.3 & +2 & +0.7 \\
\hline $\begin{array}{l}\text { Energy resolution } \\
\qquad(\sigma / E) \text { at } 50 \mathrm{GeV}[\%]\end{array}$ & 4 & 20 & 4 & 20 \\
\hline $\begin{array}{l}\text { Typical position } \\
\text { resolution } \\
\text { at } 50 \mathrm{GeV}\left[\mathrm{cm}^{2}\right]\end{array}$ & $0.2 \times 0.2$ & $2 \times 2$ & $0.2 \times 0.2$ & $3 \times 3$ \\
\hline Characteristic & 0.9 & 0.8 & 0.7 & 1.3 (vertical) \\
\hline $\begin{array}{l}\text { width of azimuthal } \\
\text { boundary region }[\mathrm{cm}]\end{array}$ & & & 3.2 & 3.2 (hor izontal) \\
\hline
\end{tabular}


Table 3 Properties of the Vertex Time Projection Chambers.

\begin{tabular}{ll}
\hline Wire Organization & 8 modules, 16 octants/module, \\
& 24 wires/octant, 24 pads/octant \\
Inner Layer Coverage & $3.5^{\circ}<\theta<176.5^{\circ},-3.5<\eta<3.5$ \\
Outer Layer Coverage & $8.7^{\circ}<\theta<171.3^{\circ},-2.6<\eta<2.6$ \\
Number of Wires/Pads & 3072 wires, 3072 pads \\
Spatial Precision & $200-500 \mu \mathrm{m}(0-15 \mathrm{~cm} \mathrm{drift)}$ \\
2-track Resolution & $6 \mathrm{~mm} / \theta(\mathrm{z}), 6 \mathrm{~mm}(\mathrm{r}), 3 \mathrm{~cm}(\phi)$ \\
\hline
\end{tabular}




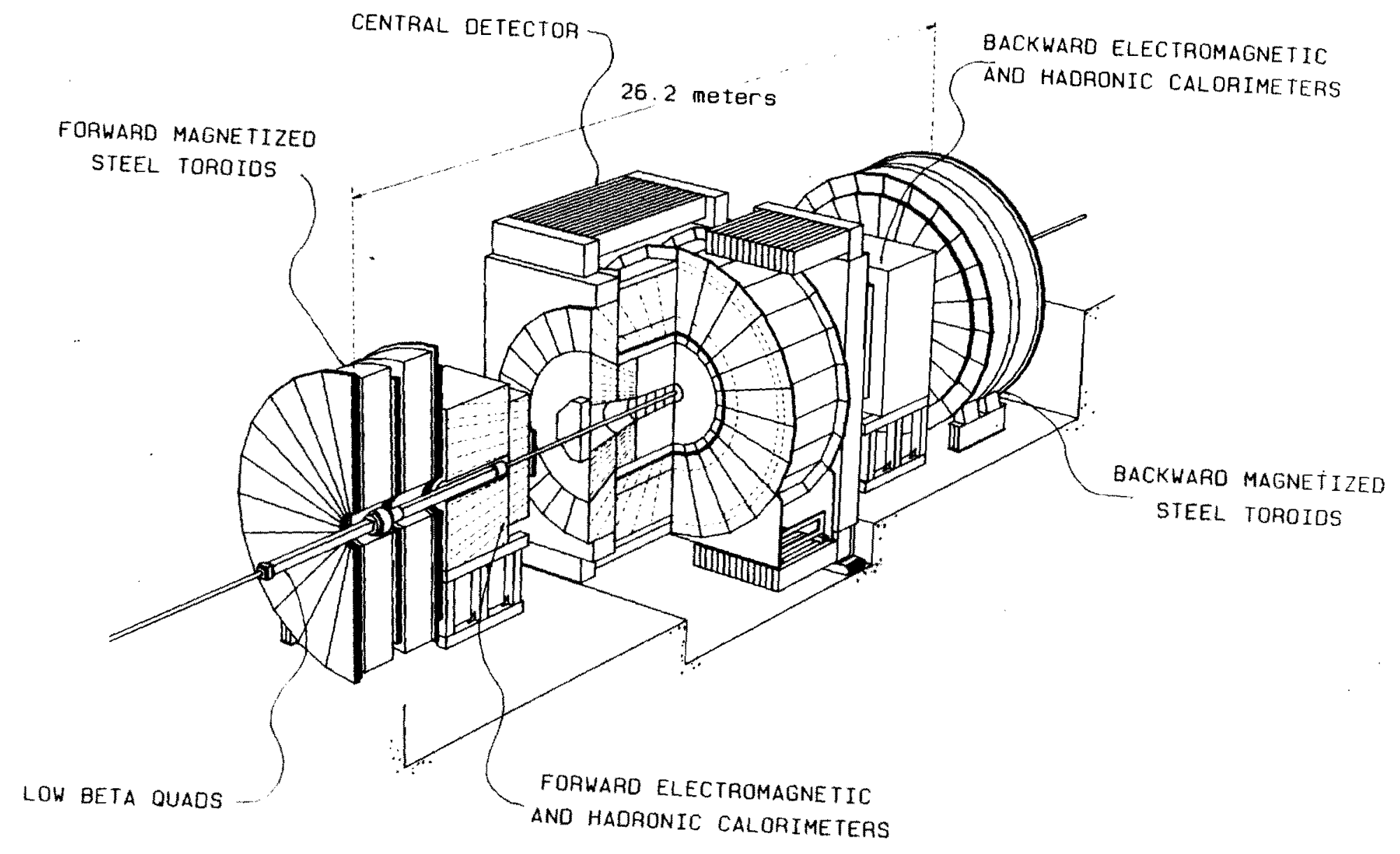

Fig. 3 A perspective view of the CDF detector. 


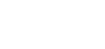

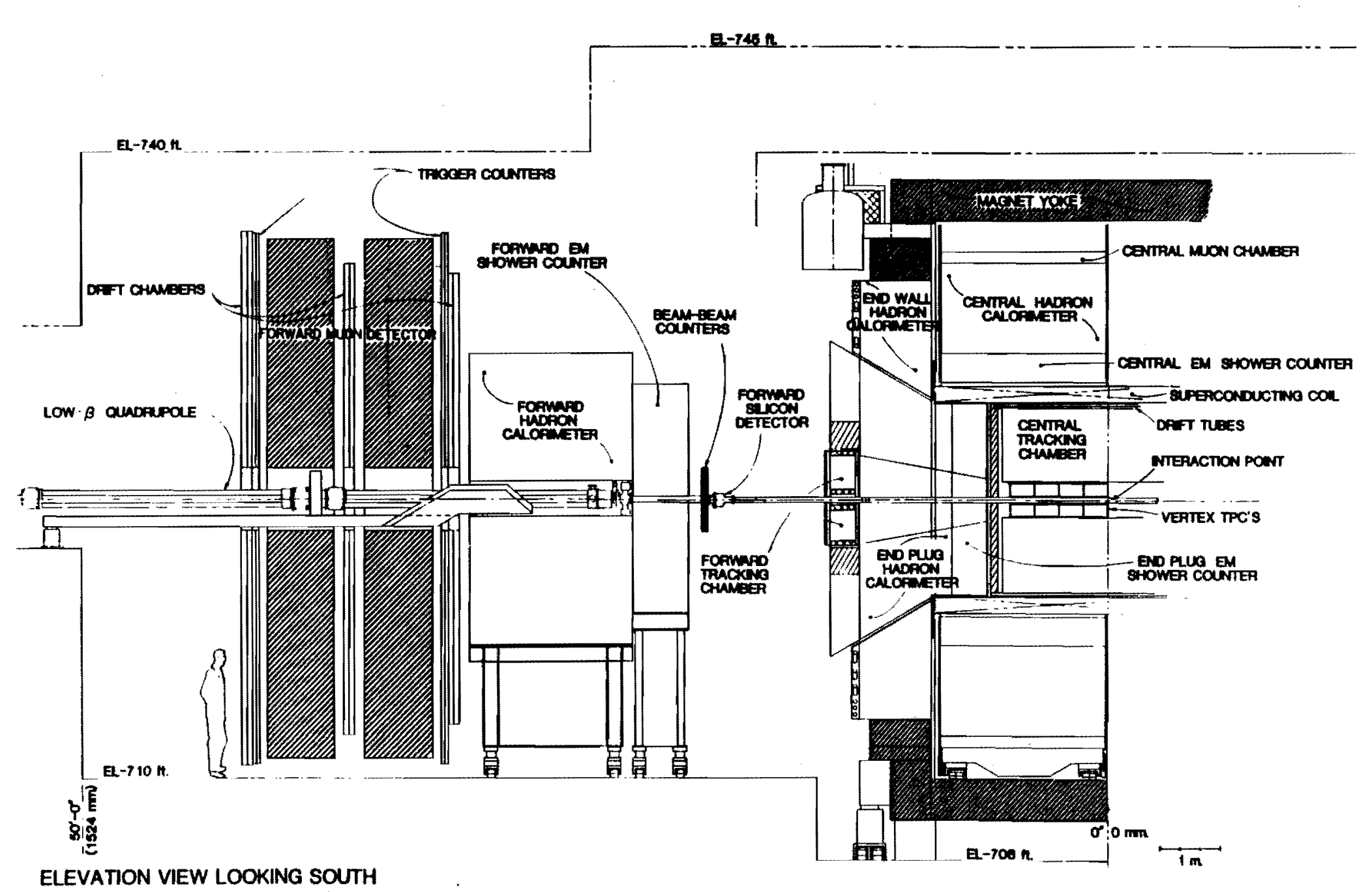

Fig. 4 A cut-away view of the CDF detector. 
HORIZONTAL VIEW OF FACILITY

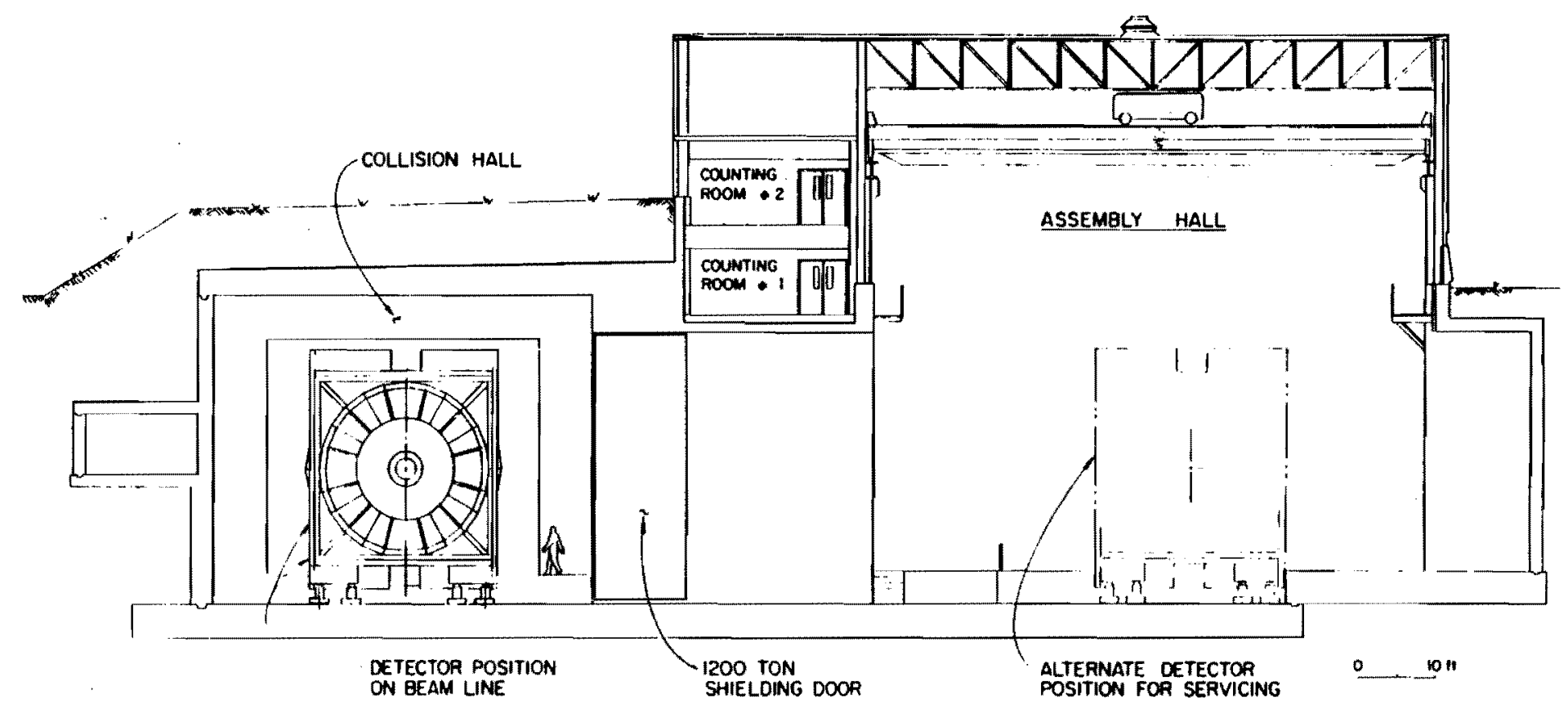

Fig. 5 The assembly building and collision hall for the detector 


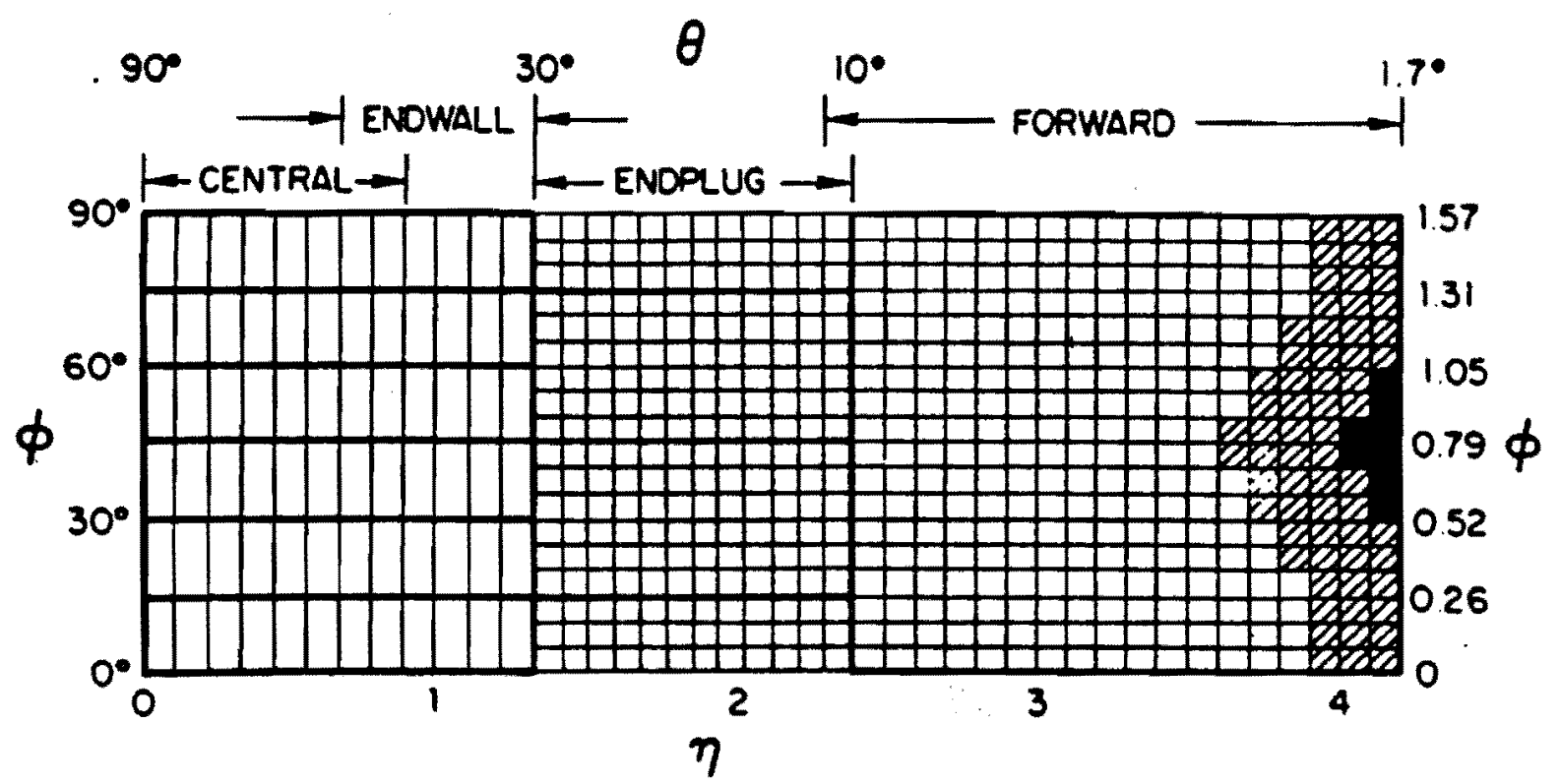

WIIC Portiol depth coveroge only due to cul for low beto quodrupoles No coveroge

Fig. 6 Boundaries of the calorimeter towers on the $\eta-\phi$ space. The coverage shown is for hadron towers; the EM calorimeters have a complete coverage out to $\eta=4.2$. 


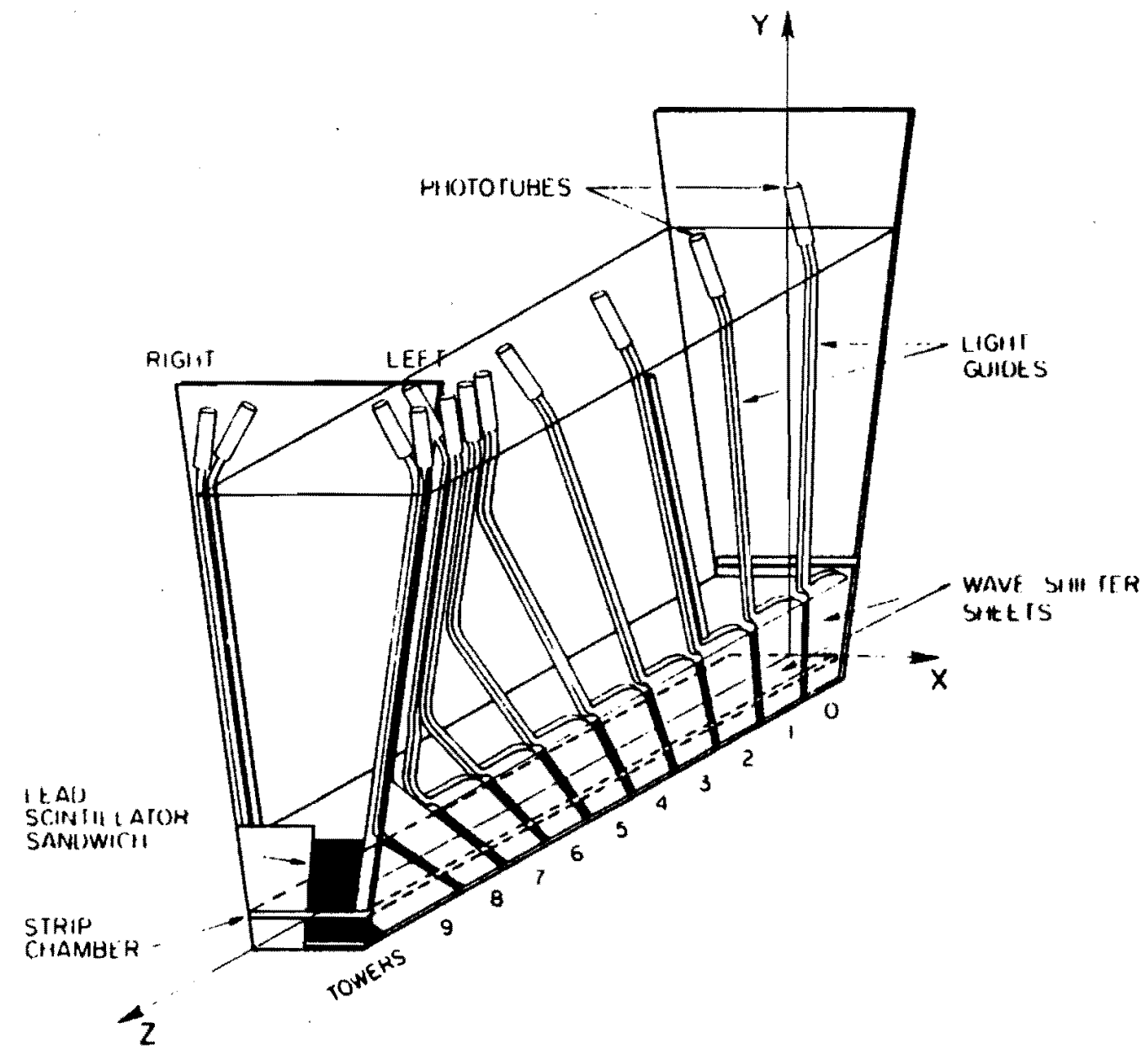

Fig. 7 A central calorimeter wedge showing the light collecting.system for the CEM. 


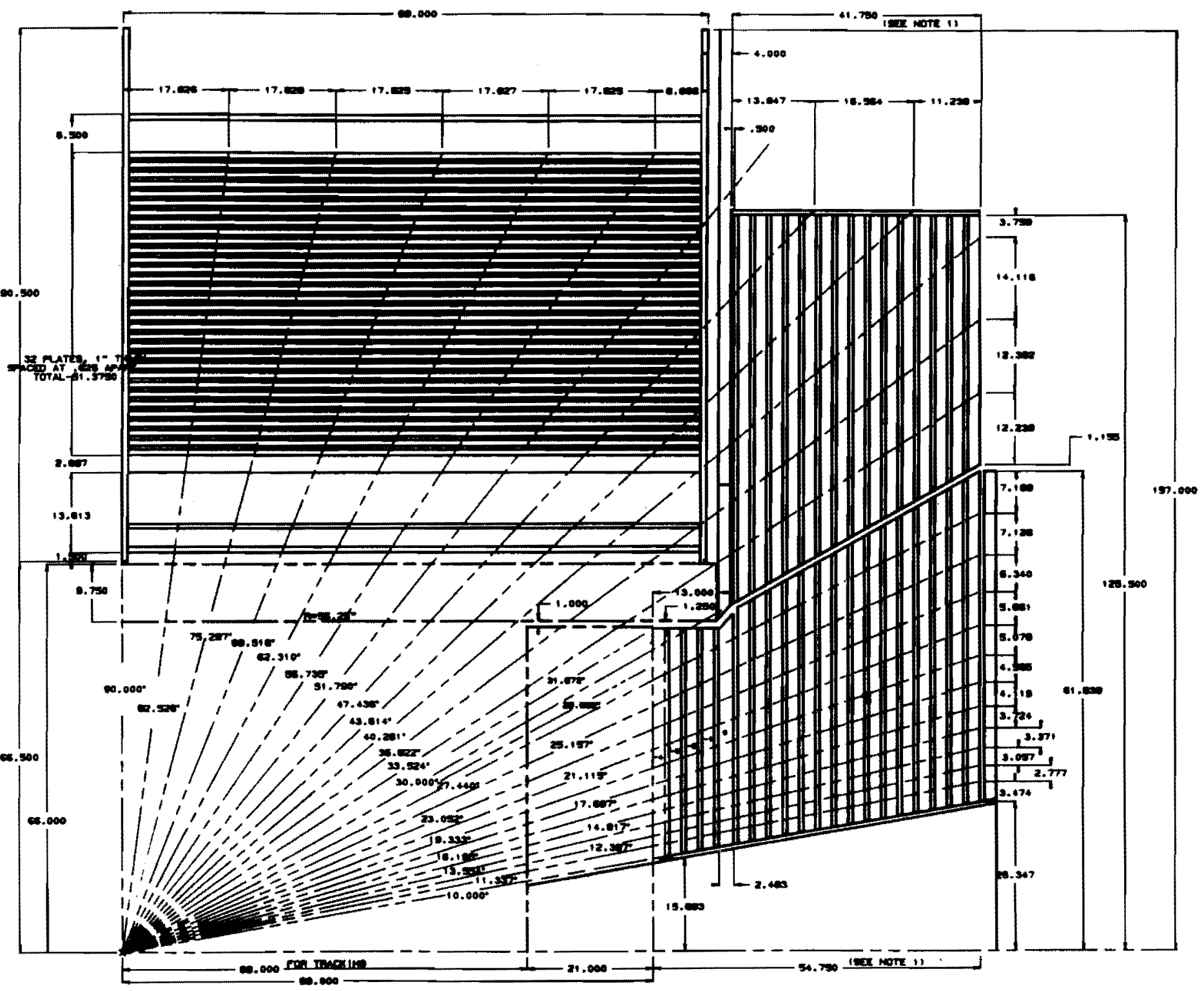

Fig. 8 A cut-away view of the central, endwall and plug calorimeters showing the tower boundaries in $\theta$. 


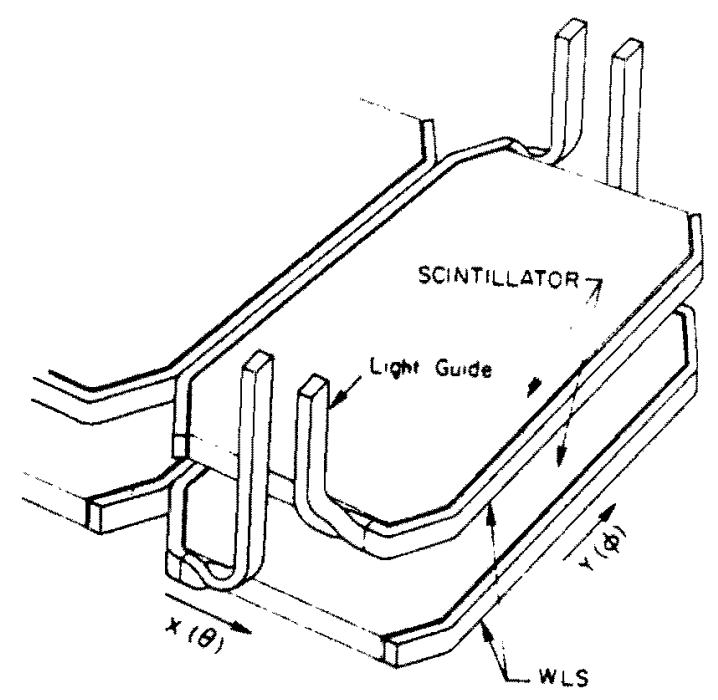

Fig. 9 Wavelength shifter strips collecting light from each $\mathrm{CHA}$ scintillator layer.

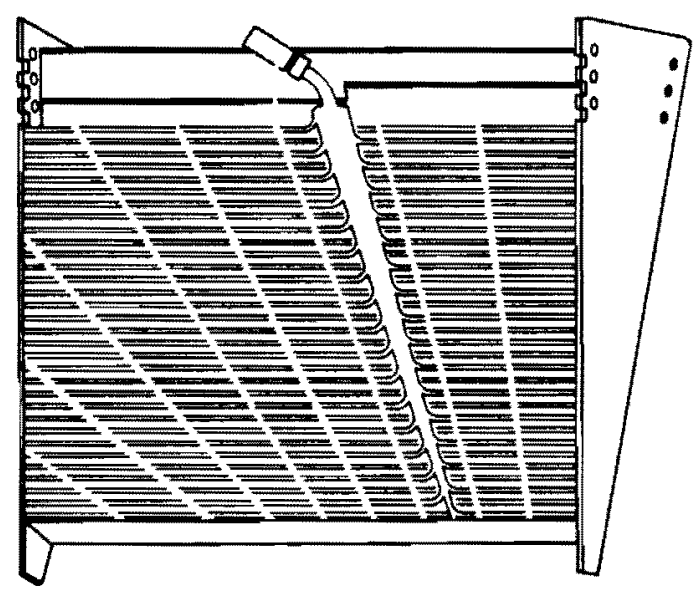

Fig. 10 A central calorimeter wedge showing the light guide for a $\mathrm{CHA}$ tower. 


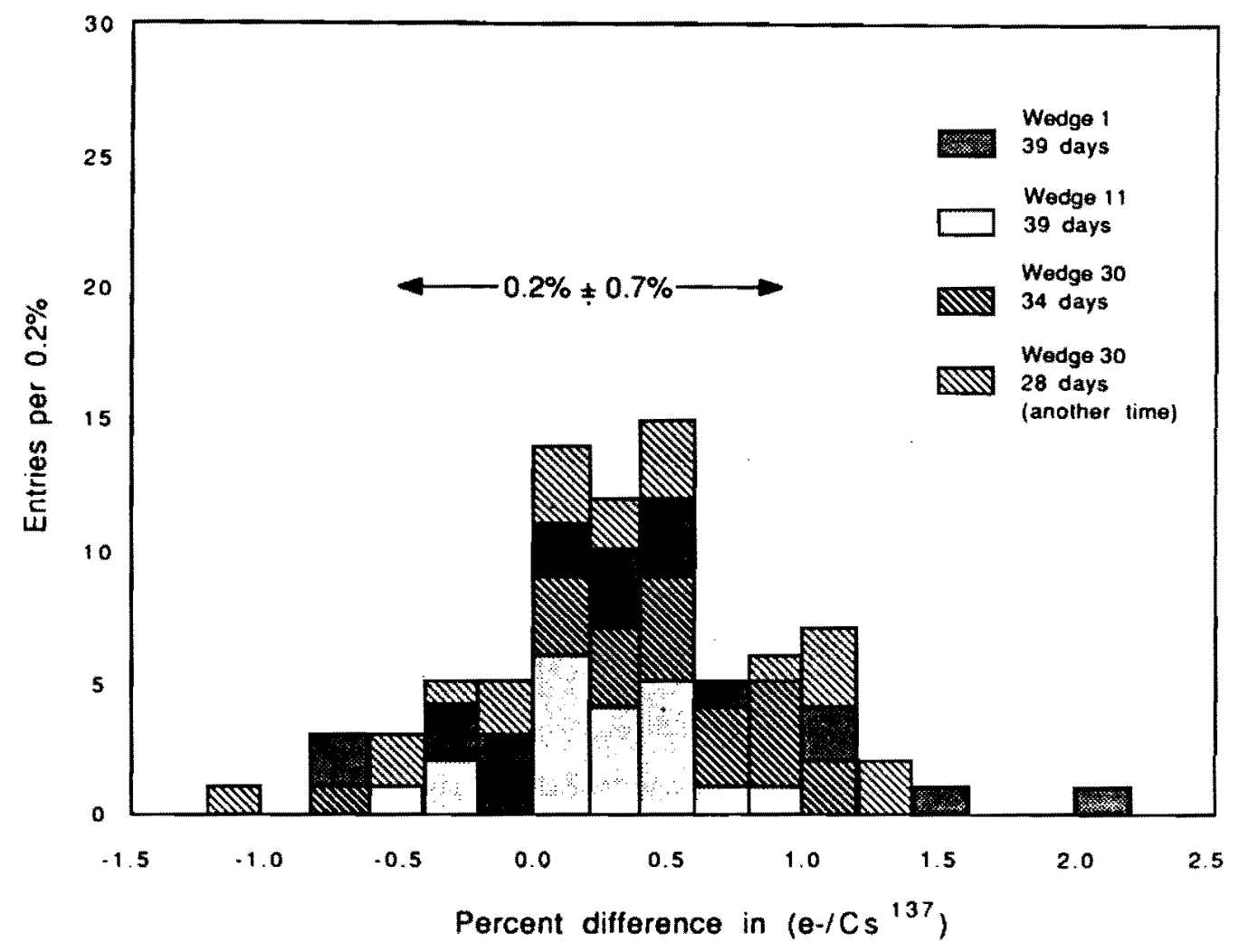

Fig. 11 Calibration reproducibility for the CEM. The difference in beam to ${ }^{137}$ Cs source ratio for each tube is plotted for successive procedures about 5 weeks apart for three modules. The deviation of the centroid from zero corresponds accurately to the source decay. 


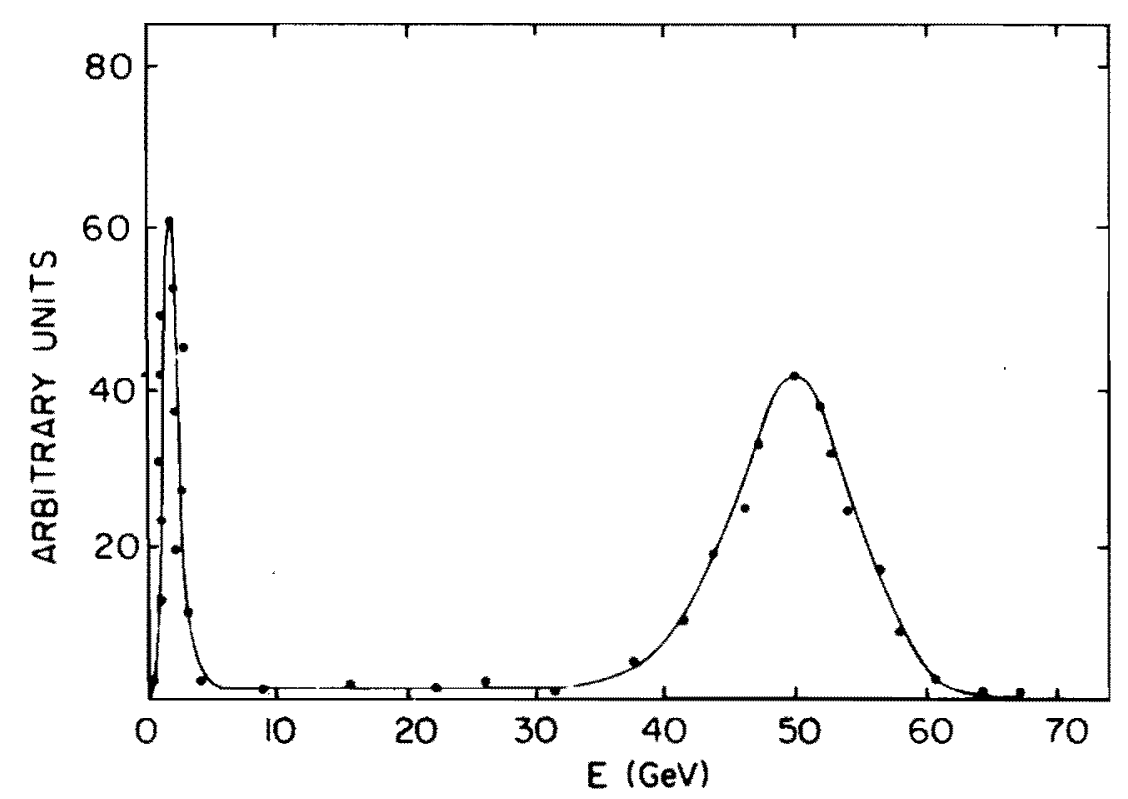

Fig. 12 Pulse height distribution for $50 \mathrm{GeV}$ pions in CHA tower 1. Only pions which are minimum ionizing in the EM calorimeter are plotted.

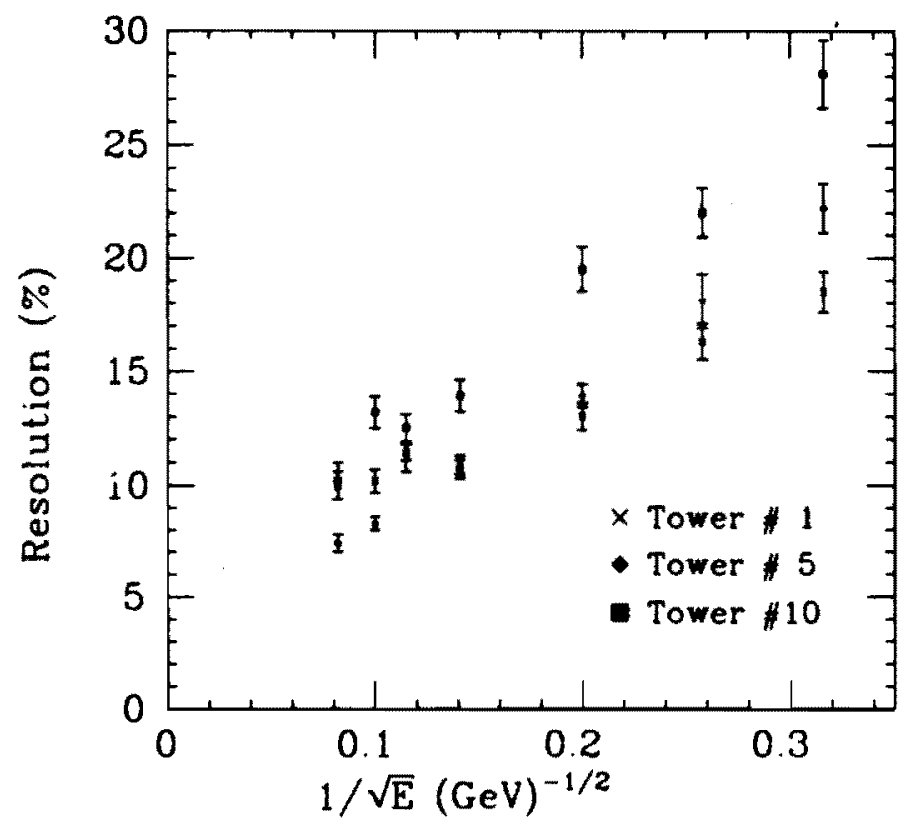

Fig. 13 Energy resolution as a function of pion energy for 3 CHA towers. 


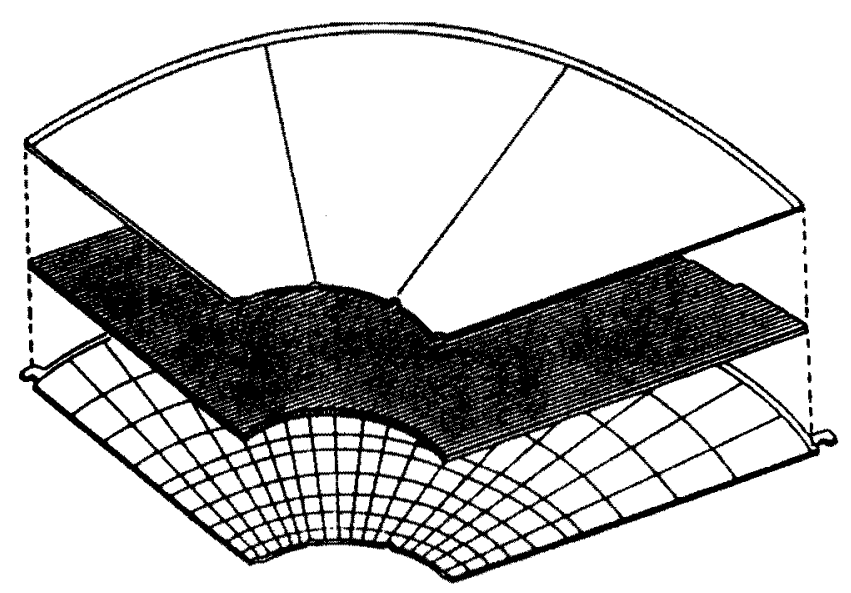

Fig. 14 Exploded view of a PEM layer, showing the proportional tube array, the PC board with pad patterns, and the PC board for the ground plane.
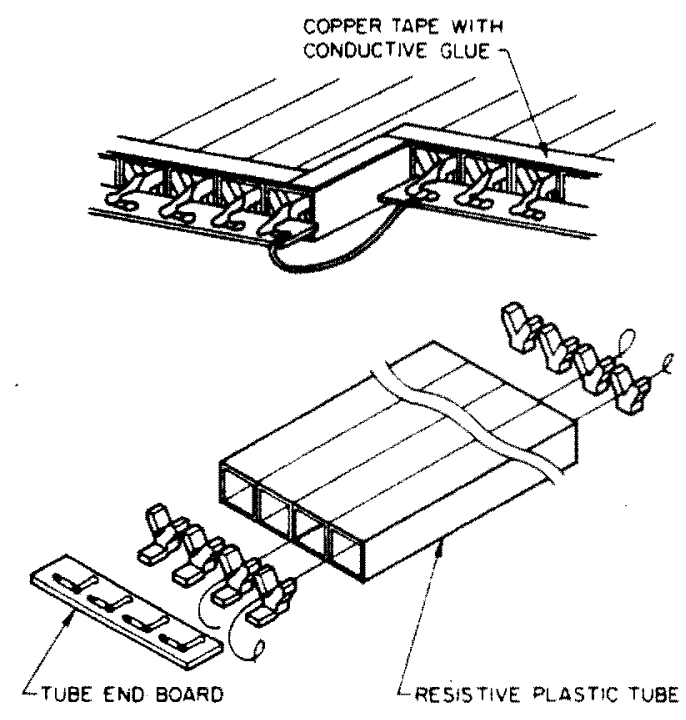

Fig. 15 More detailed view of the PEM tube array. 


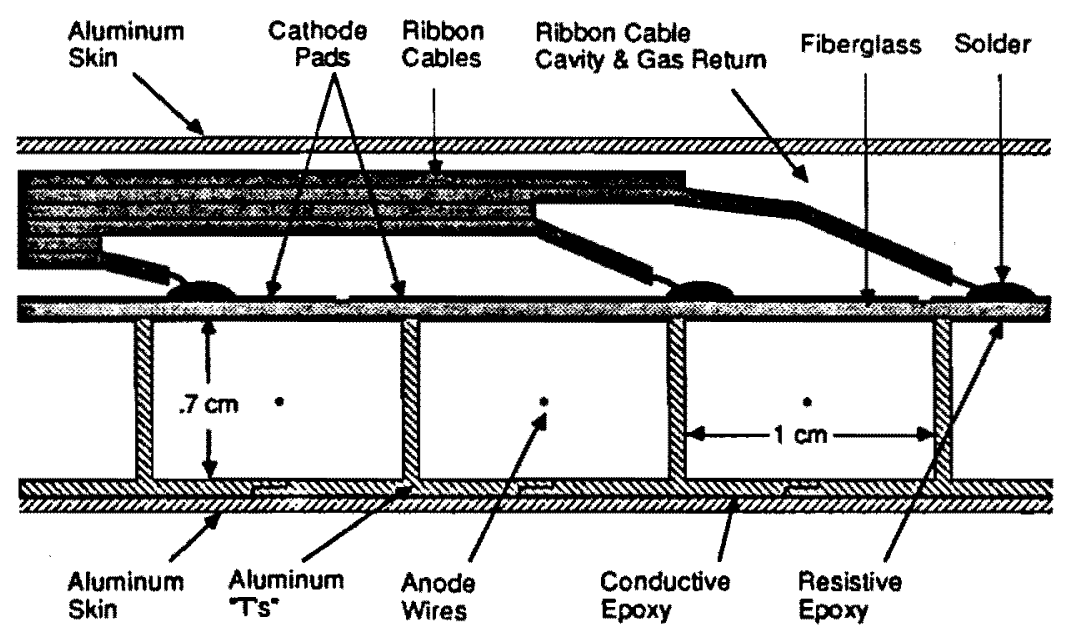

Fig. 16 Cross section of a typical FEM (gas) chamber.

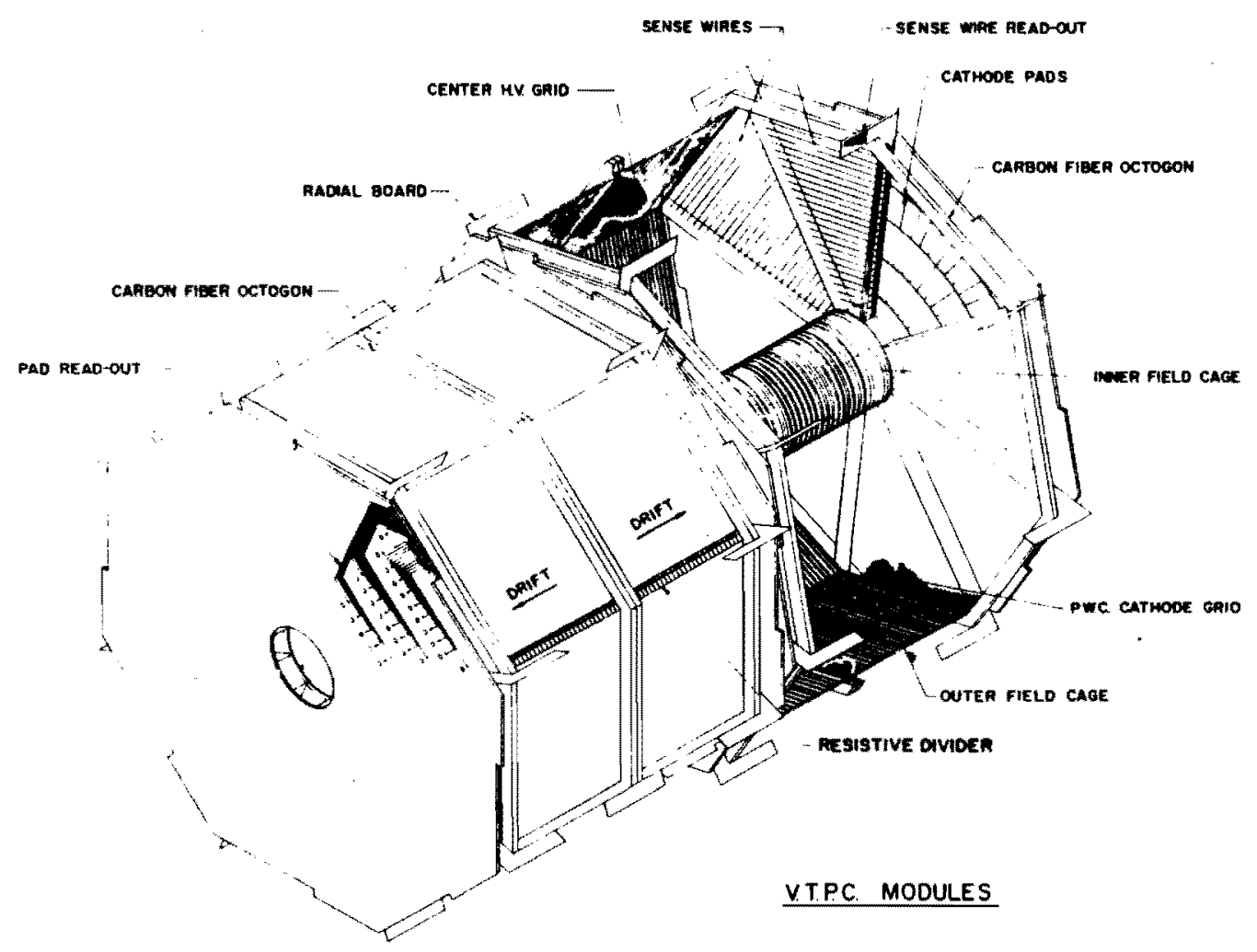

Fig. 17 An isometric view of two VTPC modules. They are rotated in $\phi$ by $11.3^{\circ}$ with respect to each other. 
CHAPTER 3

\section{THE TRIGGER AND DATA ACQUISITION SYSTEMS}

This chapter continues the description of the CDF detector in the last chapter. The electronics will be described. The trigger, built at the Univ. of Chicago, is the central brain of the experiment, deciding which events are worth recording for further analysis. At the design luminosity of the Tevatron, the rate of collision events is $50,000 / \mathrm{sec}$-- much faster than can be written to tape. The Chicago trigger has been very successful in making an intelligent selection online and earned respect.

The success of the data acquisition system (DAQ) is an amazing achievement in coordinating so many different components with so many channels (100K). In Sect. 3.1 we will describe the electronic channels associated with the calorimeters we described in the last chapter. We then have great deal of description of the Chicago trigger as it was during the 1987 run (Sect. 3.2). Sect. 3.3 and 3.4 are about the sequence of data readout and network organization.

\subsection{Front End Electronics (RABBIT) [27]}

There are two types of scanners in the CDF readout system. One is the SSP (SLAC Scanner Processor) which is Fastbus based and responsible for the readout of the trigger and all the tracking systems $(40,000$ channels). The other is called the "MX" which is interfaced to Fastbus through a MEP (Multiple Event Port) module and is responsible for all the remaining systems $(60,000$ channels).

A block diagram of the readout associated with the MX's for the central calorimeters is shown in Fig. 18. Besides the MX's, which are located in the first floor counting room, the RABBIT (Redundant Analog Bus-Based Information Transfer) system includes modules contained in 129 RABBIT crates which live side-by-side with their respective detectors. Since access is very limited, every crate is equipped with two sets of power 
supplies, bus lines and crate digitizers (EWE) to insure that if one of them fails the other can continue the operation. In the following we will give brief explanations of the meaning and functions of modules in the diagram.

a) PMA: the photomultiplier QVC (charge to voltage converter)

It is a 12 channel charge-integrating amplifier module used in the photomultiplier system of the central calorimeters. It has an accuracy of better than $1 \%$ over the energy range from $300 \mathrm{Mev}$ to $375 \mathrm{GeV}$, requiring a dynamic range of approximately 100,000 . Each channel has a charge sensitive amplifier and sample-and-hold circuitry (Fig. 19) implemented with $x_{1}$ and $\times 16$ amplification stages. A direct analog output ("Fastout") for each channel is provided to the CDF trigger system through dedicated cables.

b) PMT: the photomultiplier TVC (time to voltage converter)

This is the hadron time-of-flight system. Each module contains eight common-stop TVC's serving a single wedge. The individual starts are the amplified and discriminated sums of two phototube dynode outputs.

c) MAT: central muon QVC/TVC

The module contains eight identical sections, each serving the two ends of a CMU drift tube. Each section contains two charge-integrating amplifiers for the charge division measurement of the position along the wire and one TVC for drift time measurement of the position transverse to the wire.

d) BAT: Before After Timer

It generates signals controlling the gating operation of the sample-andhold switches of the charge-integrating amplifiers and the start-stop and reset functions of the TVC channels. It also has a fixedprecision reference voltage, a programmable reference voltage, and special timing signals for on-board charge injection calibration and TVC test. Temperature and voltages can also be readout from the BAT.

e) EWE: Event Write Encoder

It has two functions: a) It talks with the $M X$ and performs any required digital communication with the registers of front end modules. b) It performs analog-to-digital conversion of differential voltages that are multiplexed to it by front end modules across the back plane. It tests a 
signal before conversion to determine whether it is above a software settable threshold, and performs channel by channel pedestal subtraction before readout.

f) $M X$

The MX consists of six large multilayer printed circuit boards (mounted on a 27 in.x57in. aluminum frame) interconnected with wire-wrap and containing 900 IC's. It is a programmable device with an arithmetic processor containing a high speed $A L U$ and multiplier. The RAM resident program controls the acquisition of data from the RABBIT crates, applies channel dependent corrections, and stores the corrected data in event buffers.

g) MEP: Multiple Event Port

The MEP is a Fastbus module which serves as the interface between the MX and the outside world of Fastbus, through which the MX data is routed to the device responsible for assembling the events (Event Builder). It also monitors the status of each MX buffer and receives any errors reported by the $M X$.

\subsection{The Chicago Trigger [28]}

The trigger has been built on the idea of a multi-level trigger structure to reduce the data taking rate from about $50 \mathrm{k} \mathrm{Hz}$ (at the designed luminosity of $10^{30} \mathrm{~cm}^{-2} \mathrm{~s}^{-1}$ ) to the $1 \mathrm{~Hz}$ speed of tape writing. The lower level trigger (Level 1) performs quick calculations to reduce the rate to a point (about $100 \mathrm{~Hz}$ ) where the next level (Level 2) can do a more sophisticated analysis without incurring significant deadtime (less than 10\%). If the Level-1 decision can be made in the time between beam crossings (e.g. $7 \mu \mathrm{s}$ for 3 bunch operation), then no deadtime will be suffered at this level. The minimum requirement for a Level-1 trigger is the presence of the beam-beam counter coincidence, i.e. an inelastic collision. The additional condition can be any of: a) large transverse energy Et deposition in the calorimeter, b) large Et imbalance in the calorimeter, c) the simultaneous existence of stiff tracks in tracking chambers and signals in the muon chambers, or d) others. The level-2 has about 10-50 $\mathrm{\mu s}$ to make its decision based on any of: a) number of energy 
clusters and their properties, b) the presence of stiff tracks in the tracking chambers, c) the association of stiff tracks in the tracking chambers with track segments in the muon chambers, d) more stringent requirement on the total Et or Et imbalance, or e) others. We will now proceed with the details of the CDF trigger, but only emphasize the level-1 calorimetry trigger as it was the only trigger responsible for the data used in our jet analysis. The level-2 trigger was not functional during the 1987 run. A block diagram of the CDF Level-1 and Level-2 trigger system is shown in Fig. 20. All trigger boards are in Fastbus format and in the following the initials of their names will be capitalized.

\subsubsection{The Level-1 Calorimetry Trigger}

\section{a) The Calorimetry Trigger Towers}

The ground work for the calorimetry trigger is the formation of two arrays of EM and hadronic trigger towers each subtending 0.2 in $\eta$ and $15^{\circ}$ in $\phi$. Each EM or hadronic array has $42 \times 24$ elements in $\eta \times \phi$. Two central calorimeter towers (4 PM tubes) adjacent in $\eta$ are added to form a trigger tower by the analog summers Tubesum in the trigger room. The analog inputs ("trigger fastouts") to Tubesum are brought up directly from the front end PMA boards through $200 \mathrm{ft}$ dedicated cables. Since the gas calorimeters have finer segmentation $\left(5^{0}\right.$ in $\left.\phi\right), 6$ towers have to be summed and the summing is done directly on the front end amplifier boards (PMA equivalent for gas systems). Before being summed, each gas tower energy is weighted by the tower $\sin (\theta)$ (i.e. $E$ is transformed to $E t$ ) using appropriate resistor values. The $\sin (\theta)$ weighting for central towers is done by adjusting gains in the trigger (see next section on RAW).

\section{b) Receive and Weight (RAW) Boards}

The signal for each trigger tower, either from a Tubesum (central) or a front end amplifier board (gas), is received in a RAW channel. Each RAW board contains 24 channels for a single $\Delta \eta=0.2$ slice of either EM or hadronic towers. They are distributed among ten Fastbus crates which they share with the same number of Compare and Sum (CAS) boards and one Crate 
Sum (CS) board per crate. The receiving amplifier inverts with a gain of three and its output is input to a dual multiplying DAC with output

$$
v_{\text {out }}=0.5\left[\left(V_{\text {in }}{ }^{X W}\right) 256+(0.5 B) / 256\right] \text {, }
$$

where $W$ is a weighting factor and $B$ a bias factor between 0 and 255 . The weighting factor accommodates gain variations and generates the $\sin (\theta)$ for converting $E$ to Et for the central towers. The adjustable bias is used to compensate for calorimeter amplifier offsets. By setting $W$ to 0 , we can also vary $B$ to simulate inputs for trigger testing. The output of the DAC then goes through an amplifier with a gain of 4 and an emitter follower to be sent across a short jumper cable to the RAW's companion board CAS.

RAW contains protocol and registers for all Fastbus communications with RAW and CAS for control and setting parameters. It also has a 8 bit fast DAC which under the control of Fastbus or the Crate Sum generates a voltage on CAS as the reference voltage ("tower energy threshold") for the comparators. An on-board correction circuit maintains linearity and full scale range for the fast $D A C$.

\section{c) Compare and Sum (CAS) Boards}

Each CAS board has 24 channels in a one-to-one correspondence to the channels on the companion board RAW. CAS sums all towers that are above the programmable threshold during Level-1 or as indicated by the "Cluster Finder" (section 3.2.3a) during Level-2. Each analog signal from RAW is fed to an analog switch and a comparator which compares the signal with a programmed reference (threshold) voltage produced by the fast DAC on RAl. The result of the comparison is loaded into a 24-bit "sum register" which controls the analog switches directing the tower signals into summing amplifiers. Three types of sums are formed: a linear sum, a sum weighted by sin $\phi$, and a sum weighted by $\cos \phi$, using adjusted resistor values. The "sum registers" can also be written to by the Cluster Finder during level-2 so that only towers belonging to a cluster are summed. There are four sets of sum circuits on each channel of CAS, so that processing with four different tower thresholds can be done in parallel. At level-2 time this parallelism facilitates high speed operation. Each of the twelve $(=3$ sums $\times 4$ thresholds) sum circuits is monitored by a "zero detection" circuit which 
allows for correction of any input offset bias by ramping an offset DAC and monitoring the state of the zero crossing comparator.

A 24-bit register known as the "inclusion register" is used as a mask to either inhibit, allow, or force participation of specific towers in the summation by enabling, disabling the register, or loading the inclusion register into the sum register.

A simplified schematic showing the operation of the RAW and CAS is in Fig. 21.

\section{d) Crate Sum (CS) Modules}

In each analog crate there is a set of CS boards making sums at the crate level. The first of the three boards uses high speed operation amplifier circuitry to produce four sets of Etsin $\phi$ and Etcos $\phi$ sums from the analog signals coming from all CAS boards in the same crate. The second makes sums of $E t, E t\left(\eta-\eta_{0}\right)$, and $E t\left(\eta-\eta_{0}\right)^{2}$, where $\eta_{0}$ is the mean rapidity in that crate. The use of $\eta_{0}$ is to optimize the resolution of the fast FDAC's which digitize these sums upon command from the Timing Control board. The CAS signals are shipped to the CS through a specially designed fourteen layer auxiliary backplane. The digital output from the four sets of sum circuits passes through a multiplexor, with the selected set passing to the third Crate Sum board which performs the digital arithmetic to produce twos-complement values of Et, Etsin $\phi, E t \cos \phi, E t \eta$ and Et $\eta^{2}$. This digital board also counts the number of calorimeter towers contained in a level-2 cluster, passes all Timing Control signals to RAW, CAS and CS, and contains the Fastbus protocol and all registers of the Crate Sum.

\section{e) Interceptor Boards}

The Interceptor boards intercept the Crate Sum outputs on their way to the Level-1 Sum (during level-1) and the "Listmaker" (during level-2). By recording the CS outputs in RAM's, they hold information that would otherwise be lost before readout. This is important for testing and debugging the trigger system. In addition, an event can be played back at full speed to test all downstream components of the trigger. 
f) Level-1 Sum Boards

The sums from the five EM and five hadronic Crate Sums go through Interceptor boards to the Level 1 Sum boards. There is one Level 1 Sum board for each of the quantities summed during level 1: Et, Etsin $\phi$ and Etcos $\phi$. These boards were built by the author.

The higher 10 bits of the Crate Sum values are clocked into a set of multiplexor(mux)/latches by a strobe from the Timing Control. The second set of inputs to the mux/latches come from Fastbus for testing. The outputs of mux/latches feed a set of digital adders. Each board creates grand sums of EM, hadronic and total transverse energy over the entire calorimeter. These grand sums are compared to programmable thresholds, and a bit is set if the corresponding sum is above threshold. The level 1 summation and comparison are done four times for the four different sets of summers (for different tower thresholds) in (AS. The sums and results of comparison are stored in RAM's from where they can be read out via Fastbus before the next event comes to flush them out. The output of the Level 1 Sum board consists of four bits. Each bit is the logical $O R$ of the EM, hadronic and total energy comparison to threshold corresponding to one set of tower threshold and grand sum threshold. These four bits are sent to the final decision logic, FRED, to be used in the level 1 decision.

\subsubsection{Other Level 1 Triggers}

a) The Beam-Beam Counters (BBC)

The beam-beam counters consist of two planes, each of 16 time-of-flight scintillation counters (Fig. 22), on either side of the interaction region. The counters are one inch thick and are arranged in four quadrants of four counters each. Each counter covers approximately $\Delta \eta=0.7$ and the smallest, innermost counter sits directly on the Tevatron beam pipe. The BBC system as a whole covers the region $3.2<|\eta|<5.9$. Both $B B C$ planes are $591 \mathrm{~cm}$ away from the nominal collision point.

Each counter is viewed by two phototubes. The outputs from the two tubes are fed both to a TDC and the two inputs of a mean-timer channel. The TDC's are read out through Fastbus for use in the offline analysis. The 32 
signals emerging from the mean-timers perform the trigger function of the $B B C$. These signals are latched at two separate times, one is within the time window at which particles from the beam-beam interaction are expected to reach the counters, and the other corresponds to the (early) time when beam halo particles may make hits. All the 64 latch bits are read out with each trigger. Fast out signals from the latch are combined in a fast logic board to make the final BBC signal, e.g. (East in time) (West in time), which is used for the level 1 trigger decision by FRED. The BBC timing resolution is 200 ps.

\section{b) Others}

There are other level-1 signals from the small angle silicon hodoscopes (inside the beam pipe, for elastic and diffractive events), the fast CTC track finder and the forward and central muon systems. All signals are sent to FRED to be included in the final level 1 decision.

\subsubsection{Final Decision Logic (FRED), Cross Points and Trigger Supervisor}

The CDF trigger allows the detector to be partitioned into up to 16 independent experiments at a time. These are up to 12 "autonomous" partitions intended for calibration and debugging with the trigger signals provided by the subsystems, and up to 4 "CDF" partitions reserved for physics runs using the Level-1 and Level-2 triggers. Each partition is assigned, through a set of switch boards called Cross Points (built by the author), to a Trigger Supervisor (TS) which controls the readout of the partition. The interface between the TS and the trigger is handled by two kinds of FRED boards called "CDF FRED" or "Autonomous FRED", according to the type of partition they serve. FRED is also responsible for keeping track of deadtime generated by each partition.

The Level-1 sequence is initiated by the TS sending a Level-1 Query signal to FRED. At the end of the level-1 cycle, FRED receives up to twelve Level-1 input signals and generates a Level-1 accept/reject using a look-up table stored in a $4 \mathrm{~K}$ RAM. The twelve inputs include the four bits from the Level-1 Sum, and bits from the beam-beam counters and other systems. Each partition has four look-up RAM's which can be independently rate-limited to 
rates between $0.15 \mathrm{~Hz}$ and $9.1 \mathrm{kHz}$. The final level-1 decision is the logical OR of the outputs from the four RAM's. FRED sends a Level-1 accept signal to the corresponding TS and a trigger busy signal to all TS's, thereby preventing a reset of the front end electronics.

Upon the sending of Level-1 accept, the Level-2 process is initiated. In the 1987 run Level-2 accept was automatically generated for every Level-1. When a trigger was accepted at level-2, the TS generated a start scan broadcast to the front end electronics, beginning readout of the event.

\subsubsection{Trigger Timing Control (TC)}

The coordinator of all the level-1 and level-2 sequences is the Timing Control, which gets its reference from the Master Clock. The TC consists of two types of boards, a Master and 10 Slaves (one for each crate).

The TC Master is a $50 \mathrm{MHz}$ progranmable sequencer which generates the address of the current instruction which is used by both the Master and the Slaves. The Master has a $16 \mathrm{~K}$ by 64 bit memory, specifying the dwell time of the current instruction, the next address for jump instructions, the sequencer's instruction. It also can select a bit from one of the Slaves for use in branch conditions. The Slaves each have $16 \mathrm{~K}$ by 32 bit memory which contain the strobe information. Each Slave can also receive up to 8 status bits back from the module it controls. These bits can then be multiplexed to the Master where they are used for conditional jumps.

The Timing Control also stores tower threshold values which must be loaded serially, one threshold every 80-100 ns, through the Crate Sum to the fast DAC on RAW. During level-2, TC assembles a 64-bit word of cluster information which is used by the Level-2 Processors.

\subsection{The Dnline Data Acquisition System (DAQ) [29]}

The DAQ pipeline is shown in fig: 23. The master controlling the flow of data is the Buffer Manager (BFM). The communication sequence between the BFM and the other members in the pipeline is shown in Fig. 24.

To start operation of the DAQ system the user must define a partition by "booking" the detector components (actually the corresponding MX and SSP 
scanners). After partition-wide initialization and downloading of parameters, gains, and pedestals, a DAQ sequence is repeated at every Tevatron beam crossing unless it is prohibited by an on-going sequence. The sequence is started by the Master Clock which is synchronized once per revolution to a marker obtained from the Tevatron Beam Synch Clock. The timing information is generated continuously in the form of a Clear and Strobe (C\&S) pulse with the leading edge (Clear) and the trailing edge (Strobe) timed to the bunch crossing. As shown in Fig. 25, the distribution of C\&S pulses to the front end electronics follows two paths. A tightly timed version of C\&S is cabled directly from the Clock to the Gate Selector crates located on the detector components. Another path is through a series of Crosspoints (XPT) and Trigger Supervisors (TS) such that a TS has control over the generation of C\&S to the detectors within a partition. Upon receiving C\&S, the TS sends a Level-1 Query to start the Level-1 and Level-2 trigger system if the trigger is not busy.

If an event is accepted by Level-2, the TS will send the START SCAN broadcast message to the front end scanners. When the scanning is completed and one of the scanner buffers is ready to be read out, a BUFFER FULL signal is sent by the TS to the Buffer Manager. The BFM then instructs the Event Builder (EVB) to pull out the event from the scanners and acknowledges the TS when the pullout is done, freeing the TS for next beam crossing. The EVB reformats scanner data into banks which are organized by detector component and have the format expected by the VAX tape logger and consumer processes.

\subsection{Network Organization [29]}

Fastbus was chosen for the CDF data acquisition system for its supposed flexibility in supporting both high speed devices (synchronous transfers) and low speed devices (asynchronous full handshake transfers), and the protocol with multiple bus masters which allows parallel processing and partitioning of the system.

The Fastbus network is shown in Fig. 26. The UNIBUS Processor Interface (UPI) is the Fastbus interface to the host VAX computers. The QPI is a $Q-$ 
Bus version of this interface for the Micro-VAX's. There are two categories of segments, the cable segments and the crate segments. The communication between two independent Fastbus segments is provided by bidirectional ports, the Segment Interconnects (SI). The SI modules on the same cable segment are daisy-chained together. For the 1987 run, CDF used 53 crate segments which contain most DAQ modules, 16 cable segments and 66 SI modules. The majority of segments and modules are used by the front end electronics and the Level-1/Level-2 trigger system. 


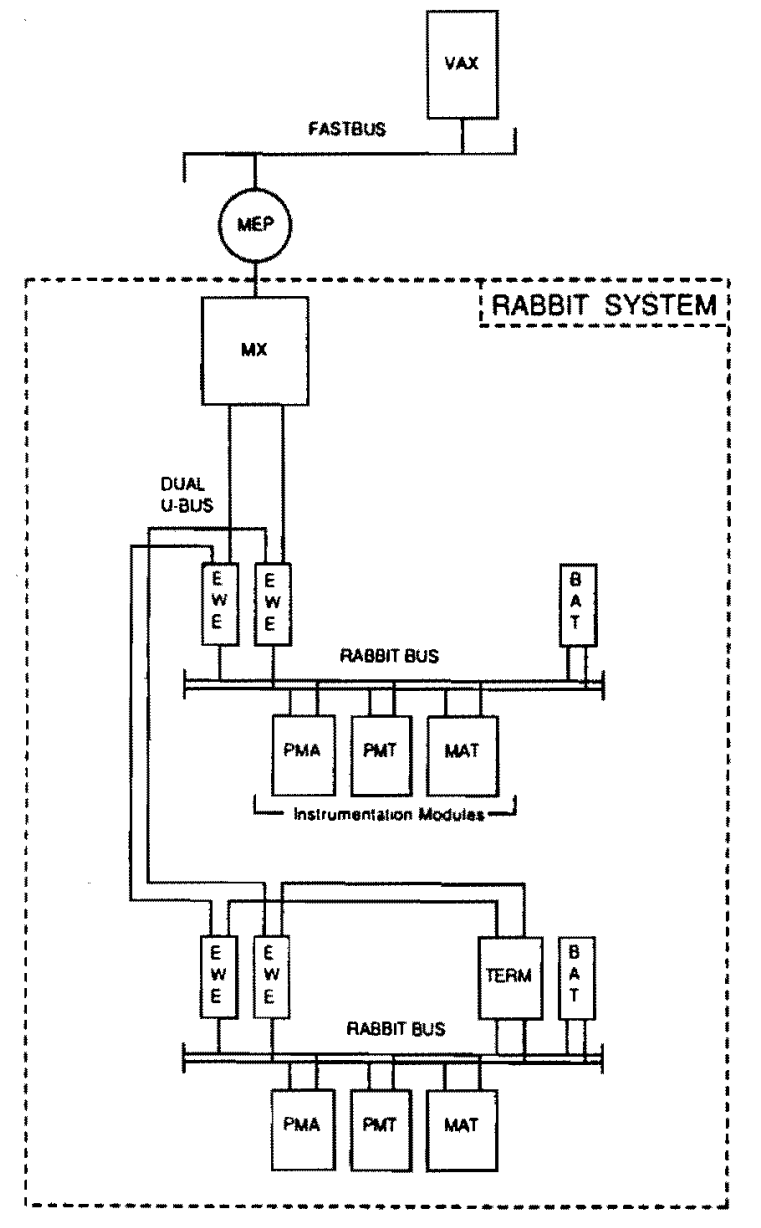

Fig. 18 A Block diagram of the CDF readout system -- RABBIT.

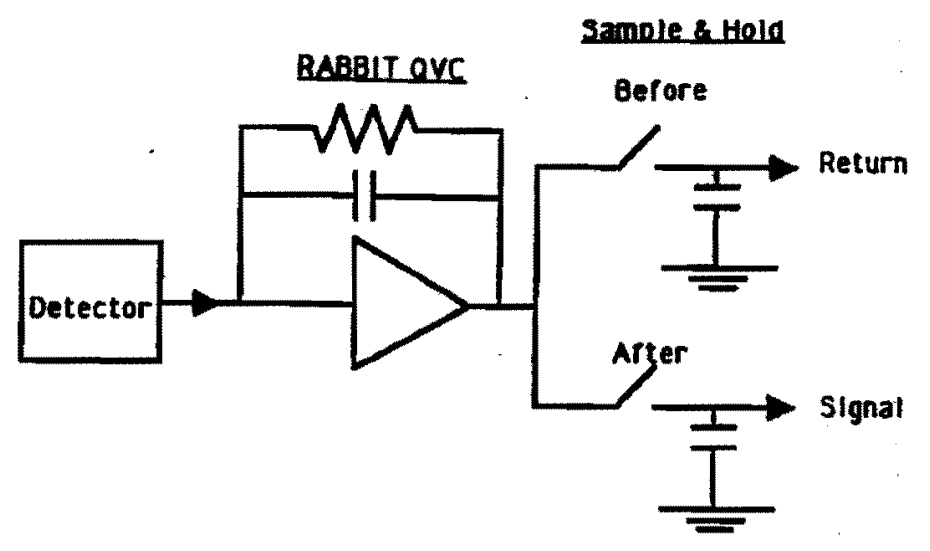

Fig. 19 A charge-to-voltage convertor and a sample $\&$ hold circuit.

I I


CDF Trigger System

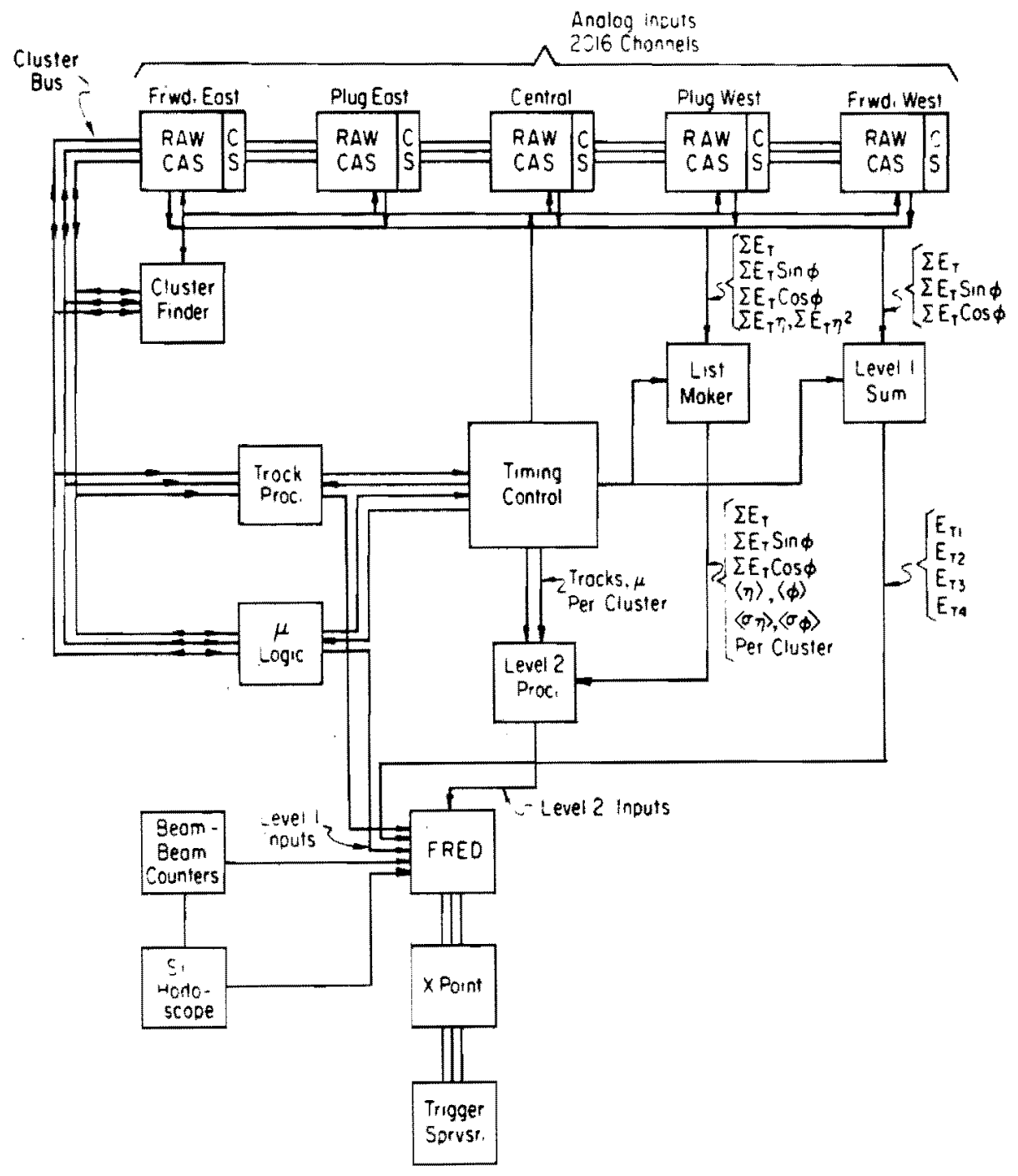

Fig. 20 A block diagram of the CDF Level-1 and Level-2 trigger system. 


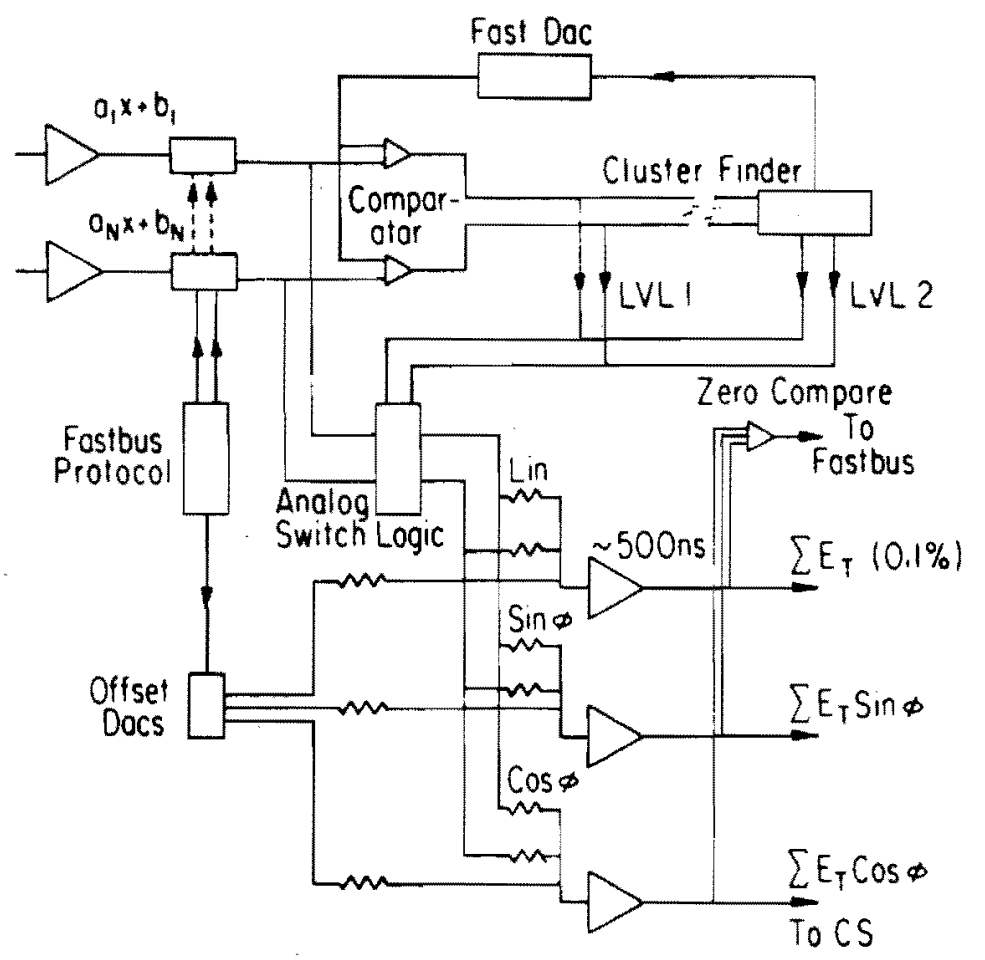

Fig. 21 A schematic showing the operation of the Receive and.Weight and Compare and Sum boards. 


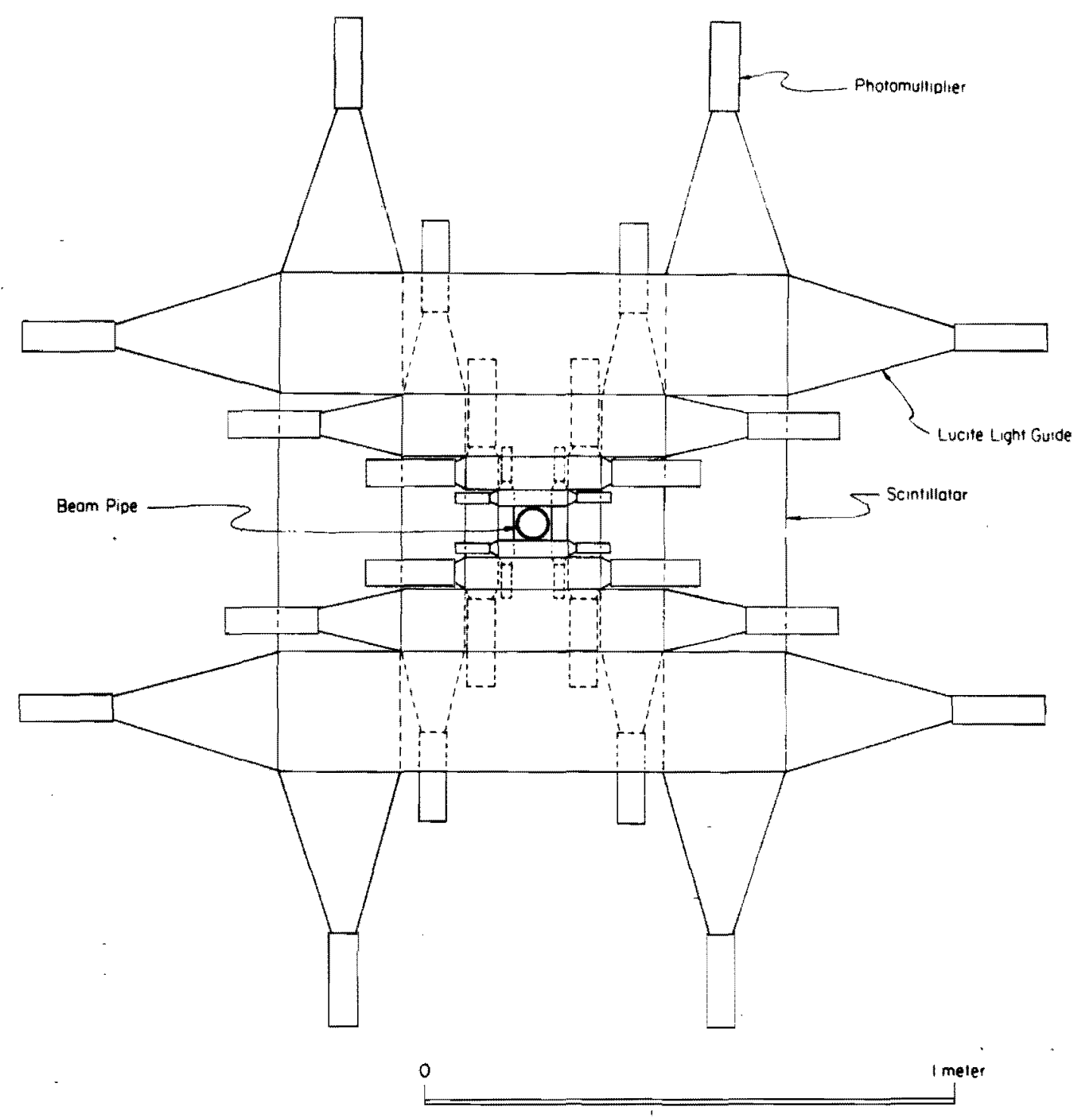

Fig. 22 One of the Beam-Beam Counter arrays. 


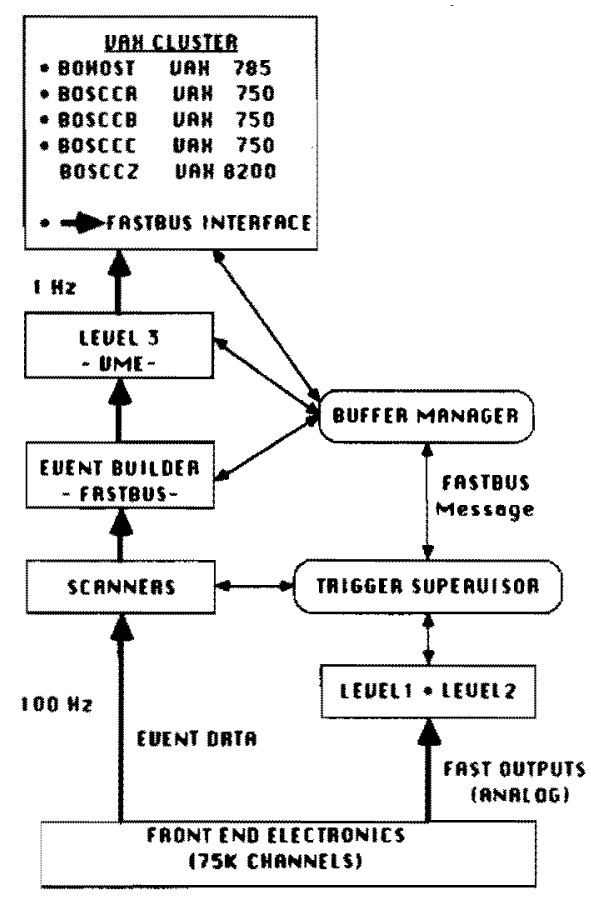

Fig. 23 The Data Acquisition (DAQ) pipeline.

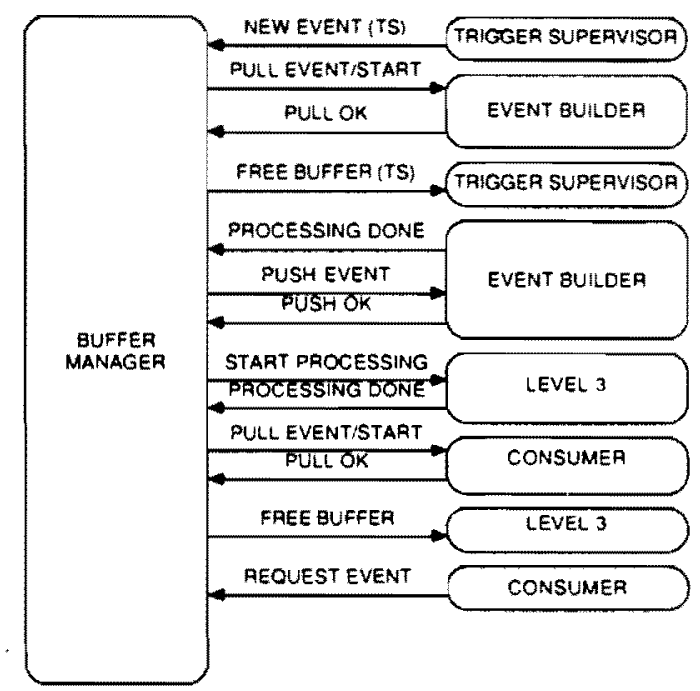

Fig. 24 The messages from/to the Buffer Manager controlling the DAQ pipeline. 


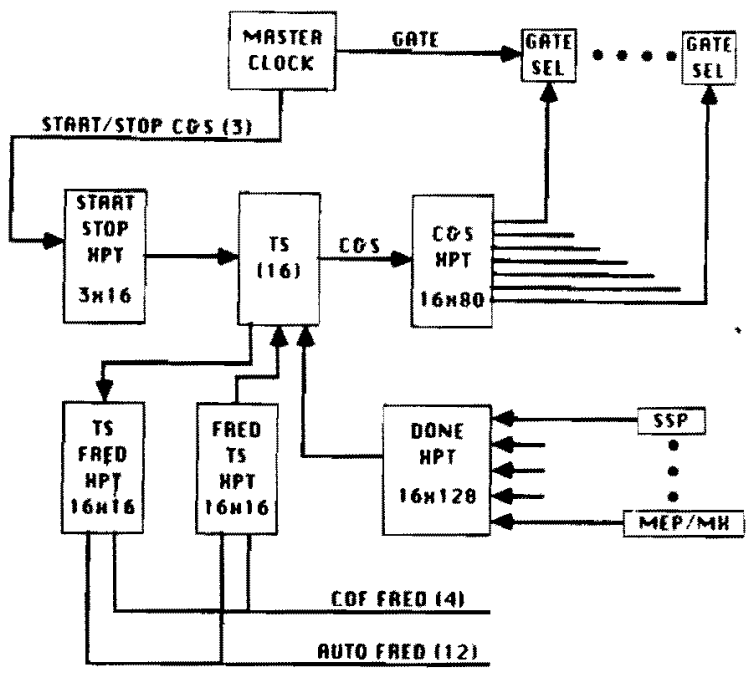

Fig. 25 Crosspoint modules -- electronic switch boards.

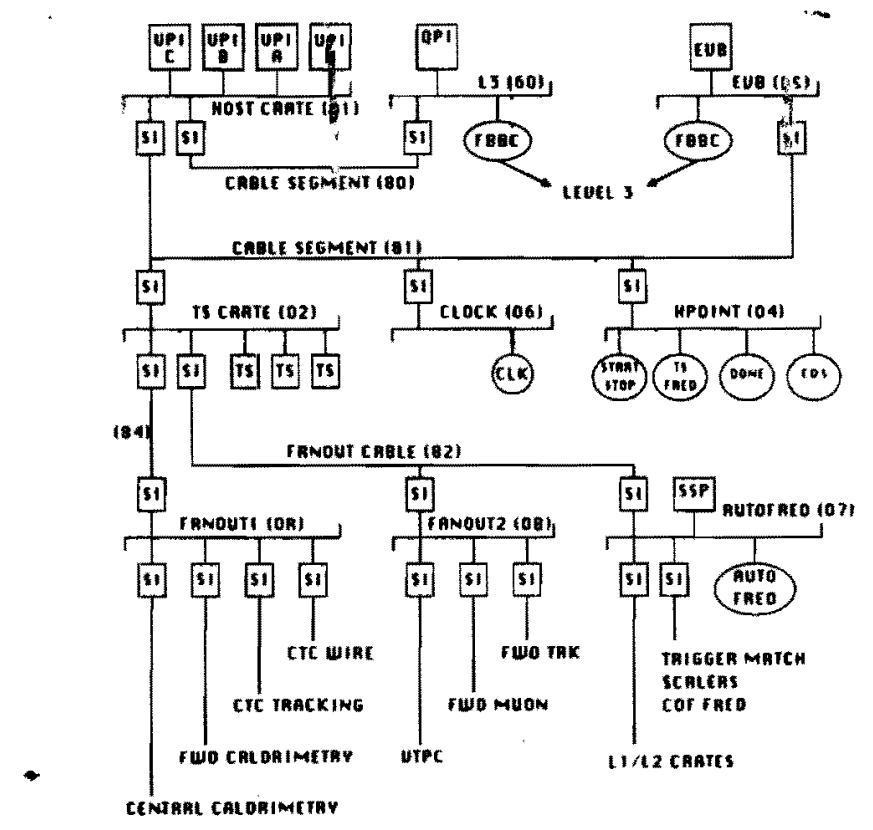

Fig. 26 FASTBUS network. 


\section{CHAPTER 4}

THE 1987 RUN

\subsection{The Performance of $\bar{p}$ Source and the Tevatron Collider}

The first CDF data run took place from January to May, 1987. This was a successful engineering run both for the Tev $I$ ( $\bar{p}$ source and collider) and the CDF detector [30]. The peak luminosity delivered by the Tevatron reached $1.6 \times 10^{28} \mathrm{~cm}^{-2} \mathrm{sec}^{-1}$ near the end of the run (Fig. 27). The integrated luminosity delivered by the Tevatron was $72 \mathrm{nb}^{-1}$, and $33 \mathrm{nb}^{-1}$ (with 15\% uncertainty) was recorded on tape (Fig. 28). Here we have used the luminosity measured by the CDF Beam-Beam Counter system (BBC) assuming the total inelastic non-diffractive cross section seen by the $B B C$ is equal to $44 \mathrm{mb}$.

There were more than 900 hours of $p \bar{p}$ collisions and only 8 accesses for repairs. By the end of the run (the last 40 days) the typical 'day' consisted of a 4 to 8 hour setup time for antiproton transfer, followed by an 12-20 hour collider store. The mean luminosity lifetime was 8 to 12 hours during the first 8 hours of the store, and 10 to 16 hours during the second 8 hours. The luminosity loss was mainly due to beam emittance growth and in small part due to loss of beam current. When the luminosity had decayed sufficiently, the stores were often terminated by CDF choice rather than by failures as was the case during the early period of the run.

There is an intricate "shot setup" procedure to extract $\bar{p}$ bunches from the accumulator $r i n g$ : reverse inject into the Main Ring (typical efficiency $=87 \%)$, accelerate to $150 \mathrm{GeV}(\epsilon=88 \%)$, coalesce Main Ring RF buckets into a single bucket $(\epsilon=70 \%)$, inject from MR into Tevatron $(\epsilon=72 \%)$, accelerate to $900 \mathrm{GeV}(\epsilon=99 \%)$, and low- $\beta$ "squeeze" $(\epsilon=90 \%)$. The typical overall efficiency was $34 \%$. The Tevatron was in 3-on-3 bunch operation. 


\subsection{The CDF Performance}

\subsubsection{Detector Components}

The detector was essentially complete for the 1987 run except for: a) half of the sampling planes in the forward hadron calorimeter; b) the Level-2 trigger system which does cluster finding and track matching etc. for more sophisticated triggering; 3) a level-3 trigger system of microprocessors and the associated full-rate DAQ system. There were about 70,000 channels in the detector and more than $99 \%$ of them were read out by the DAQ system via Fastbus. Fewer than $1 \%$ of channels were dead.

\subsubsection{Monitoring and Calibration}

Routine procedures were also established for monitoring, taking pedestals and calibration. These included $\mathrm{Cs}^{137}$ sources, a laser flasher, a Xenon flasher, and a LED flasher for the scintillator calorimeters. For the gas calorimeters there was a gas gain monitoring system of proportional tubes with $\mathrm{Fe}^{55}$ sources. The gas gain system ran continuously and the gain constants were downloaded every two hours (if necessary) into the trigger and readout electronics.

\subsubsection{Trigger}

The trigger was a mix with a rate of about $1 \mathrm{~Hz}$ at $90 \%$ livetime. The Level-1 trigger consisted of the following (but only (b) is relevant to this analysis):

a) A "minimum bias" trigger: made from the BBC east-west coincidence. This trigger was rate-limited to about $0.05 \mathrm{~Hz}$. The $\mathrm{BBC}$ coincidence is also required for all the other triggers.

b) A jet trigger: made from the total Et deposited in all the electromagnetic and the central hadron calorimeters. The hadron gas calorimeters were excluded due to various noise problems. The total Et threshold was varied from $20 \mathrm{GeV}$ to $45 \mathrm{GeV}$ depending on the luminosity. See section 8.2 for more details about the thresholds and the corresponding data, as this trigger was responsible for the data we used for the central jet analysis. 
c) An electron trigger: made from the total Et deposited in the EM calorimeters. The total EM Et threshold was varied from 10 to $20 \mathrm{GeV}$. Also at least 1 tower with Et $\geq 7$ to $12 \mathrm{GeV}$ was required.

d) A central muon trigger: made from the coincidence of a central muon chamber track with a high $\mathrm{Pt}$ track (Pt $>5$ to $10 \mathrm{GeV}$ ) in the central tracking chamber found by a fast track processor.

e) A high Pt muon trigger from the forward muon system. This trigger was rate-limited to about $0.05 \mathrm{~Hz}$.

\subsubsection{Data Taking Efficiency}

About 500 raw data tapes were produced with some 1200 events per tape. The average CDF data taking efficiency was $46 \%$, but rose to $66 \%$ by the end of the run. There was detector readout deadtime of a few percent and time for tape changes, run initialization and gas gain downloading. An additional $15 \%$ of store time were lost in "Main Ring Blanking". This was to gate off the detector during certain parts of the 2-second Main Ring $\bar{p}$ production cycle. 


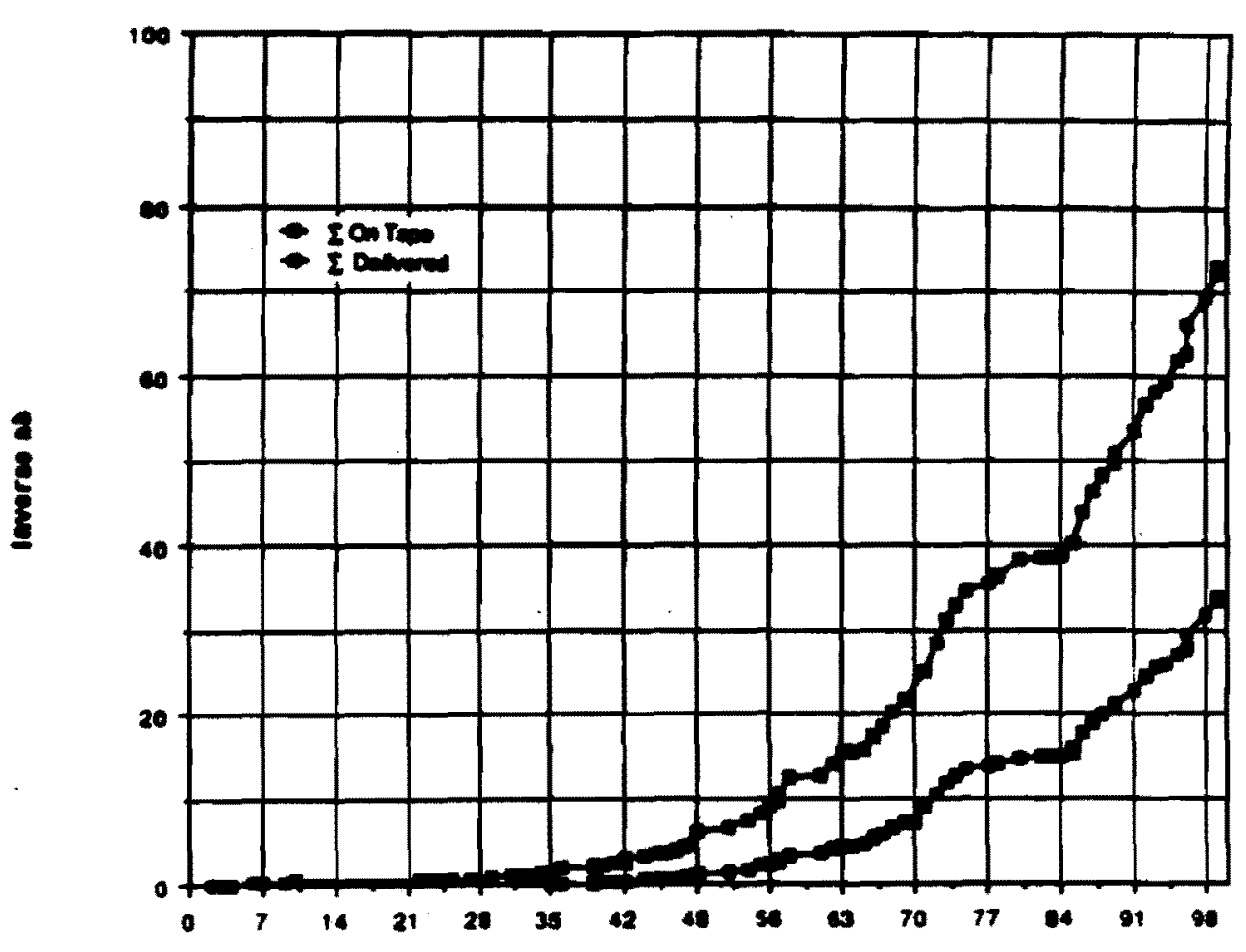

Doy ( vecinning Fob. 1, 1we )

Fig. 27 Tevatron peak luminosity $\left(10^{28} \mathrm{~cm}^{-2} \mathrm{sec}^{-1}\right)$ for Feb. 1 - May 11, 1987. Each point represents a separate $\bar{p}$ p store. 


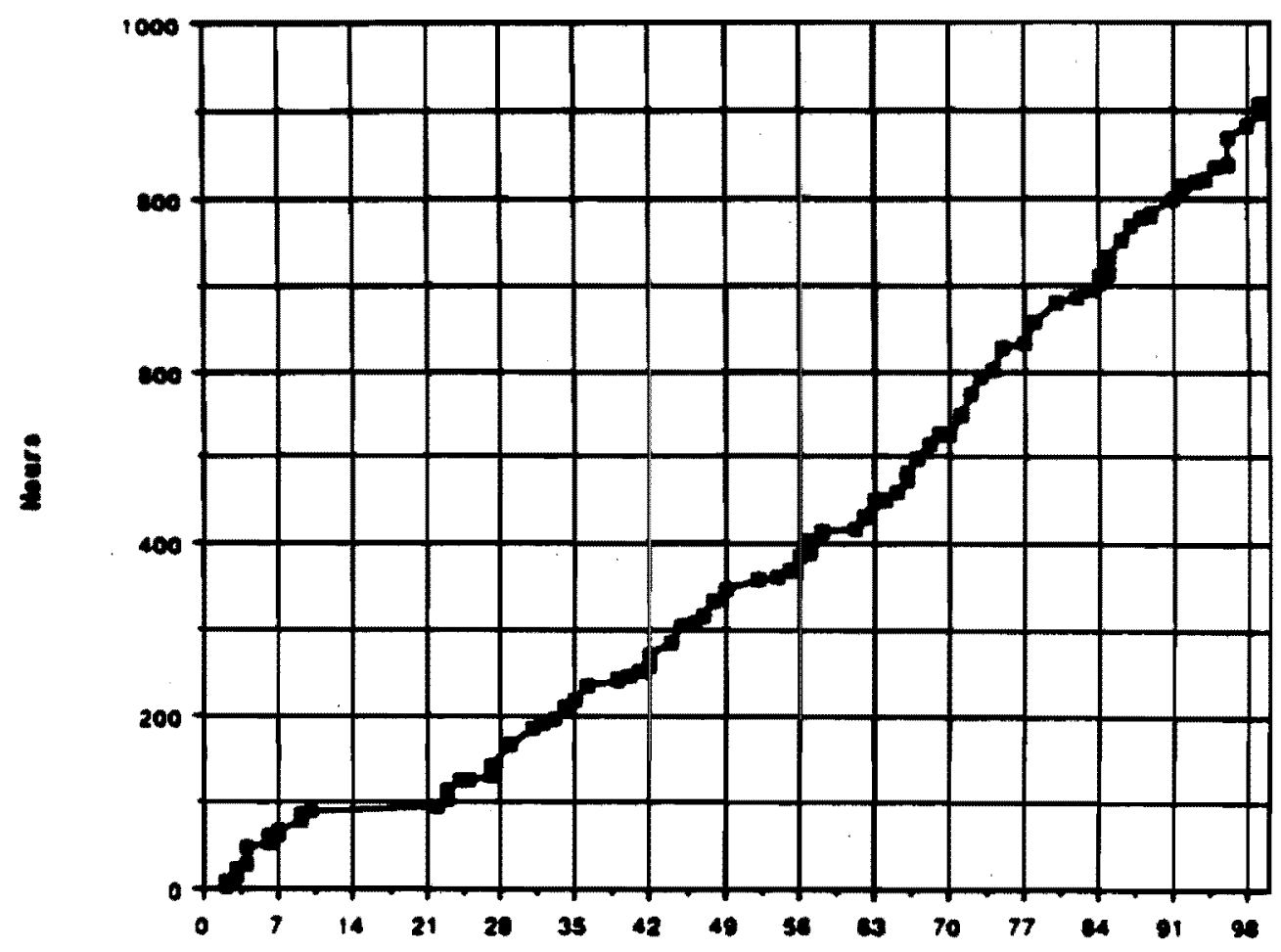

Dey ( bainning Fes. 1. 1807 )

Fig. 28 The integrated luminosity $\left(n b^{-1}\right)$ versus time for the 1987 run. The top curve is the delivered Iuminosity, and the lower curve is the Iuminosity written to tape. 


\section{CHAPTER 5}

\section{DATA CLEANUP}

With a system as complicated as CDF, where wholesale is the only way to do business, there are bound to be multiple problems both during the time of data taking and in the data taken. We dedicate this chapter to the calorimetry problems only and explain how they are removed one by one. The cleanups are described in various CDF notes and the following sections contain no more than those notes.

\subsection{The Spikes in CEM, CHA and WHA [31]}

In these central calorimeters showers are sampled by scintillators, light guides and photomultiplier tubes as described in Sect. 2.2. Dccasionally particles produced by $\bar{p} \bar{p}$ collisions, particle showering or other sources, hit the light guides and generate Cerenkov light to fake a large energy deposition in one of the two tubes looking at each tower. There were also discharges between the photo cathodes and the magnetic shields or the nearby transition pieces (or the paper wrapping of transition pieces). But since each tower was looked at by two tubes, single tube noise is easy to identify by the imbalanced tube energies.

Define the energy in the two tubes to be $E_{0}$ and $E_{1}$. The energy in each tower is given by

$$
E_{\text {tower }}=\sqrt{E_{0} E_{1}}
$$

If $W$ is define to be the width of the module and $\lambda$ to be the attenuation length of the scintillator in $\mathrm{cm}$, then the shower center (relative to the center of the wedge and in units of the wedge width) is given by

$$
x=\frac{1}{2}-\frac{\lambda}{2 W} \log \left(E_{0} / E_{1}\right) \text {. }
$$

Since the energy collected in a given tube is proportional to the number of photo-electrons, the fluctuations are Poisson and should vary approximately 
as $\sqrt{E_{0}+E_{1}}$. Such fluctuations can result in the shower position as calculated above displaced from its true position. The algorithm to suppress spikes looks for a deposition which has the calculated position outside the physical boundaries of the wedge by more than some number of $\sigma$. In the case where one tube has zero energy, the total energy in the tower is calculated by $E=\max \left(E_{0}, E_{1}\right) / 2$ when $\max \left(E_{0}, E_{1}\right) / 2<1 \mathrm{GeV}$, and $E=0$ otherwise. For the case when both tubes have non-zero energy, a spike is defined by

$$
\left|\log \left(E_{0} / E_{1}\right)\right|>R+N_{\sigma} K / \sqrt{\left(E_{0}+E_{1}\right) / 2}
$$

where $R=W / \lambda$ for the CHA and WHA or 0.7 for the CEM, $N_{\sigma}$ is the number of $\sigma$ 's to cut on and $K$ is a constant measured for each calorimeter.

\subsection{Cosmic Rays and Stray Particles from the Main Ring [32]}

The old Fermilab $400 \mathrm{GeV}$ accelerator Main Ring, now serving for the production of $\bar{p}$ and as the booster for the Tevatron, ran unshielded 5 feet above the CDF detector. During some parts of the 2 sec Main Ring $\bar{p}$ production cycles, e.g. proton injection from the booster at $8 \mathrm{GeV}$ and $\overline{\mathrm{p}}$ production targeting at $120 \mathrm{GeV}$, lots of particles were lost from the beam and bombarded the top portion of the CDF detector. These stray particles deposit up to 1 Tev of energy within the $1 \mu \mathrm{sec}$ window when the calorimeters were active. Some would mimic hot tubes as described in the previous section (Sect. 5.1) by hitting the light guides. These events can hardly escape visual inspection as they have the distinct feature of having a long trail of towers lit up on the top of the detector.

Another very common background comes from cosmic rays, predominantly due to bremsstrahlung of muons. This type of background has only very few towers involved and usually the deposited energy is either purely electromagnetic or purely hadronic.

It is fortunate we have TDC's implemented in the central hadron calorimeter because the times of hits tell the nature of the event. The time distribution for CHA towers with Et $>1 \mathrm{GeV}$ from good collision events is plotted in Fig. 29 (CDF note $\$ 581$ ), where times are relative to the beam 
crossing. An in-time window $[-10,25]$ ns was chosen and any energy deposited out of this window must have come from Main Ring splashes or cosmic rays. A special run with Tevatron off and Main Ring on was taken to study the cuts for splashes. Cuts at $N=1$ and $E=10 \mathrm{GeV}$ remove more than $98 \%$ of splash events.

But with the in-time window defined above, about $5 \%$ of cosmic rays would come as in time. In this case the EM energy fraction can be cut on because almost all cosmic rays deposit either purely electromagnetic energy or purely hadronic energy. This will be discussed in Chapter 8 (Sect. 8.3).

\subsection{Cleanup for the Gas Systems}

In the dijet mass analysis we require the two highest Pt jets in an event to be in the central rapidity region. Thus the energy clusters in the plug and forward regions also affect the result, though rather weakly. There are more things to be cleaned up in the gas calorimeters than in the central calorimeters, e.g. electronic noise [33], high voltage discharge [34], neutron induced spikes [35] and pedestal shift [36]. We refer the reader to the indicated references (CDF notes) for detail. 


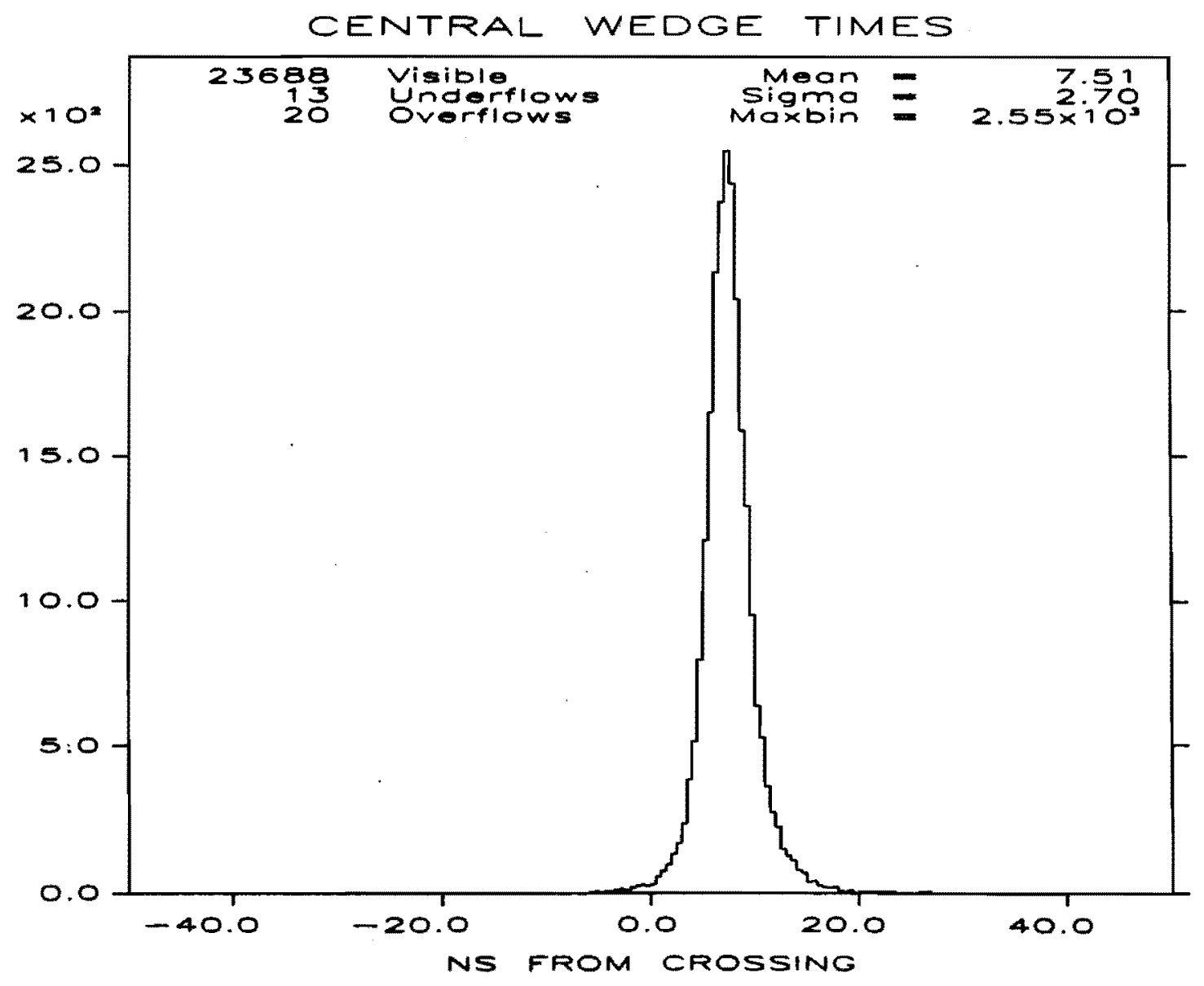

Fig. 29 Times of hits on the CHA towers. The tower Et threshold is $1.0 \mathrm{GeV}$ (from CDF581). 


\section{CHAPTER 6}

\section{CLUSTERING ALGORITHMS, JET CHARACTERISTICS \\ AND THE COMPARISON OF DATA AND MONTE CARLO}

After the channel $A D C$ counts are converted into energy and the data are fully cleaned up, we are left with the job of finding energy clusters -jets (see Ch. 1). In hadron collisions the first sign of jets was observed at ISR energy [37]. A higher density of particles in the phase space around a trigger particle, and in a direction azimuthally opposite to the trigger particle, was interpreted as a pair of scattered partons making their ways into the detector. This jet physics has been firmly established at SPPS energy [38], and has become one of the major and natural tools for tests of QCD. In this chapter, we will first describe how the jets are found in our experiment and then discuss some features of jets as observed in the CDF detector at a $\bar{p} \bar{p}$ center of mass energy $1800 \mathrm{GeV}$.

\subsection{Clustering Algorithms [39]}

Prior to any discussion about jets, the jet finding algorithm and its performance should be stated with care and respect. Not commonly shown in conferences, there are many jet events, especially the low pt ones, consisting of messy, scattered, and hard to be defined energy lumps. Thus to have a reliable algorithm is not as trivial as it may have appeared to many people.

\section{$6.1 .1 \mathrm{JCLST2}$ and JCLST3}

There were originally 2 jet-finding algorithms in CDF. Both first find smaller, separated energy lumps in $\eta-\phi$ space and then merge nearby lumps into jets. To be included into a lump, the candidate towers must have Et > $0.2 \mathrm{GeV}$. A new lump is always started from the available highest Et tower (called a seed) with Et > $1.0 \mathrm{GeV}$. One algorithm (JCLST3) collects towers around the seed by a cone of variable size depending on the lump energy 
(the higher the energy, the smaller the cone), while the other (JCLST2) grows the lump from the seed by requiring the new towers must touch (side by side or corner to corner) at least one of the already included towers. They then use the same algorithm for merging lumps. The merging algorithm starts with the available highest Et lump, using it as the center of a cone of fixed size (default radius 0.7 ) and merging all lumps within. Then the procedure is reiterated on the leftover lumps, using the highest one as the cone center, until all lumps are assigned to a jet.

There had been concerns about JCLST3 using variable cone sizes in forming lumps, and in most applications JCLST2 was a more popular one. But as time went on, more problems were unearthed in JCLST2. Dne is the presence of a pathological island jet within another jet. In order to prevent growing lumps up the Et hills, the Et ratio of the new and the old towers during the adjacency clustering was required to be less than 2.0 for the sake of jet resolution. But with this gimmick of benign intent come the island lumps that are created within other lumps. In most cases these island lumps would be merged back (lump merging) and not constitute a problem, but not always and island jets may result. The second problem is that occasionally the adjacency chains are extended far $(>\pi)$ from the jet centers beyond any physical reason. A search for a better algorithm was thus started in the CDF jet group. Two more emerged and are meant to be free of these problems. Again one (JCLST4) continues the tradition of defining lumps from connected towers, which is the same algorithm used in level 2 trigger hardware, the other (JETCLU) pushes the idea of a cone in $\eta-\phi$ space to the extreme, using a cone of fixed size. The latter has also been used in SPS experiments.

\section{1 .2 JCLST4}

The JCLST4 algorithm (modified from JCLST2 by the author) is somewhat complicated and goes like this. A tower list is made for those towers with Et greater than a certain threshold (default $0.2 \mathrm{GeV}$ ). A subset of towers passing a higher Et threshold (default $1.0 \mathrm{GeV}$ ) are called seeds. The first phase of clustering involves only seeds. A new lump always starts from the next available (not yet included in any lump) highest Et seed. The 
algorithm checks the eight neighbors (daughters) of each included seed (parent) and include them if they are seeds and have Et less than the parent seed. It stops when it finds no more and the procedure is repeated for a new lump. The lumps created in this way are monotonically decreasing in Et from the lump center outward.

Then all lumps are grown outward at same time by including more non-seed towers layer by layer. When two lumps meet each other they are merged or remain separated depending on the depth of valley. If the lower lump total Et is 5 times greater, and its peak is 2 times greater, than the Et of the meeting tower, then the two lumps remain separated.

It then merges the fully grown lumps by a scheme examining the lump Et's, $\eta$ 's and $\phi$ 's. First lumps are ordered in descending Et's, and the distances between pairs of lumps are also ordered with the shortest on top. A new jet always starts from the next available (not yet merged in any jet) highest Et lump. The algorithm then goes down the distance list searching for a pair that has one lump already merged in the jet and one not. A cone is drawn around the unmerged lump and one sees if it's surrounded by jet Et (summed from the already included lump Et's within the cone) greater than its own. If yes the lump is merged and the searching procedure is repeated starting from the top of distance list until no more lump can be included. Then the algorithm goes to create the next jet.

But sometimes two jets may have shared lumps. In this case all lumps except the two seed lumps (i.e. the highest Et lumps) are deassigned and reassigned according to closeness of lumps. The algorithm goes down the distance list several times in search of a merged-unmerged pair and assigns the unmerged one to the same jet as the merged one.

\section{1 .3 JETCLU}

JETCLU is a algorithm similar to those used by UA1 and UA2 and proves to be simple and effective. The first stage is similar to that in JCLST4 -finding lumps. All towers above a Et threshold (default $1.0 \mathrm{GeV}$ ) are ordered in descending Et's. They are tested one by one down the Et list to see if they are adjacent to any tower(s) which is already included in a lump. If yes they are included into the same lump. If not, a new lump is 
created. The tower $l$ ist is scanned several times and many lumps are formed and consist of towers monotonically decreasing in Et from lump center outward.

Then the strategy for JETCLU is quite different from all the others. It draws a big cone in $\eta-\phi$ space (default radius 0.7 ) around a lump to engulf all towers inside that cone with Et greater than a certain threshold (default $0.2 \mathrm{GeV}$ ) to form a pre-jet. The centroid of the pre-jet is recalculated from these towers (weighted by tower Et's), and a new pre-jet is made by drawing another cone around the new centroid to replace the old pre-jet. This procedure is repeated until the pre-jet centroid is stabilized.

After all pre-jets are formed, some of them might have overlapped towers. Then the fraction of the overlapped Et is used to determine if the two pre-jets are going to be merged or remain separate. The default overlap fraction for merging is $>70 \%$. If they are going to remain separate, the overlapped towers are divided according to the distances to the energy weighted cluster centers. In this merging stage, pre-jets are ordered in descending Et's and the algorithm works down the Et list with the higher Et pre-jet having the higher priority of taking over other pre-jets and becoming a even bigger pre-jet.

\subsubsection{The Comparison and Performance Tests of Clustering Algorithms}

All these algorithms have been tested by several measures [39] (see Table 4). But the most decisive test is a technique that mixes two jet events and ask if the algorithms can find the same jets as before mixing. One makes the distribution of the fraction of the time a cluster is not found as a separate cluster after event mixing as a function of $\Delta R$, where $\Delta R$ is the distance on $\eta-\phi$ space between the two clusters before event mixing. The merging distance and sharpness can then be determined from the derivatives of this distribution as the mean and sigma of the derivative distribution (resembling the error function erf). The event-mixing test favors JCLST3 most and in general cone algorithms over adjacency algorithms. But the resolution and Et stability of JETCLU are considerably 
better than JCLST3. The jet group finally decided that the JETCLU is the best one and will be the standard algorithm for jet analysis.

In Fig. 30 we compare the $\eta$ 's, $\phi$ 's, Et's and Ntower's of jets as found by JETCLU and JCLST4 in the central calorimeter with Et > $30 \mathrm{GeV}$. From these comparisons we can have some idea how CDF jets change as the algorithm changes.

\subsection{Trigger Clustering Algorithm Versus JETCLU}

The clustering algorithm used in the Level-2 trigger hardware is very similar to that of JCLST2 (and JCLST4) in that they are based on the adjacency of towers. The main difference is that there is no merging in Level-2, which thus causes trigger clusters to suffer a considerably larger energy loss. The fact that the trigger hardware requires a higher single tower threshold $(1 \mathrm{GeV})$ and treats $E M$ and HADron towers separately makes the situation even worse. In this section we compare the hardware algorithm with the offline JETCLU, as they both are essential for the jet physics in our experiment and thus it's important for us to be aware of any big differences.

We use the data sample which was taken with a total Et trigger threshold of $30 \mathrm{GeV}$ and require the jet Et (sum over tower Et's) to be greater than $30 \mathrm{GeV}$. The trigger cluster used for comparison is selected as the highest Et cluster within a cone of radius 0.7 from the (energy weighted) center of a jet. Due to the differences in the algorithms, we would expect that the trigger clusters have fewer towers (because the higher tower threshold breaks the adjacency chain more easily) and that there will be fewer clusters per event (due to a higher seed threshold). The plots in Fig. 31, comparing the numbers of clusters in the central calorimeter and the numbers of towers ( $E t>1 \mathrm{GeV}$ ) for clusters, fit these expectations well. In Fig. 32 we show the differences in $\eta$ and $\phi$, the number of trigger clusters within a 0.7 cone from a jet center, and the Et fraction of the highest cluster. From Fig. 32(d) (in which the overflow bin contains fractions equal to 1 -- no splitting), about $1 / 10$ of jets are split into two or more trigger clusters, and for the split jets the highest clusters 
have on the average $3 / 4$ of the total trigger Et (= Et sum of the trigger clusters within 0.7 from the jet centers).

The jet Et spectrum is shown in Fig. $33(a)$ as solid lines, while the trigger cluster Et spectrum is in dotted lines. The "resolution" effect of the trigger is clearly seen on this plot below $30 \mathrm{GeV}$. The Et differences are given in Fig. $33(\mathrm{~b})$ with a mean of $-5.29 \mathrm{GeV}$ and a sigma of $5.34 \mathrm{GeV}$, representing a $20 \%$ jet Et loss on the part of the trigger. To gain some feeling about the effects of trigger threshold on the jet data collection, we plot in Fig. 34 the trigger efficiencies versus thresholds for two samples of jets with Et>30 GeV and Et > $45 \mathrm{GeV}$. The efficiencies are the fractions of the whole samples that pass the trigger thresholds. The efficiency for a trigger threshold $30 \mathrm{GeV}$ on a $30 \mathrm{GeV}$ sample is $68 \%$, and a $45 \mathrm{GeV}$ threshold for $45 \mathrm{GeV}$ sample is $66 \%$. The 90\%(95\%) efficiencies are reached at $24(20) \mathrm{GeV}$ and $38(33) \mathrm{GeV}$ respectively.

\subsection{Characteristics of Jets and the Comparison of Data and Monte Carlo}

With the JETCLU (cone) algorithm for finding a jet, we will look at some features of jets as they appear in the CDF detector. We also take this opportunity to examine the goodness of our Monte Carlo, as so many physics issues depend on it. To get sensible Monte Carlo data we need a good event generator and a good simulation package specifically written for the CDF detector. It thus requires some miracles to have the Monte Carlo and the real data show the same characteristics. In next chapter, we will use ISAJET events [13] to study jet energy corrections for various losses due to cracks, leakage and nonlinear responses of the calorimeters. So it is of immediate importance to know what this ISAJET package, combined with the CDF simulation, is doing. In the following we will compare the ISAJET (version 5.20 , but with the fragmentation parameters originally proposed by Feynman and Field, see Sect. 7.2) jets and the real jets in the same kinematical region as used in our central jet analysis (Ch. 8), i.e. the event vertex less than $50 \mathrm{~cm}$ away (along the beam direction) from the detector center and $|\eta|$ of jets less than 0.7 . We only use the $30 \mathrm{GeV}$ (total trigger Et threshold) data sample and demand that the Pt of each jet 
be greater than $30 \mathrm{GeV}$ to avoid trigger bias. The ISAJET events were generated by requiring that the two highest $P t$ jets in the events are in the $|\eta|<0.7$ region and that the dijet invariant mass is greater than 70 $\mathrm{GeV}$. The comparisons are straight forward and they are in Figures $35-44$.

We first observed that the ISAJET jets are narrower than the actual jets from the comparison of the Et weighted $\eta$ and $\phi$ widths (Fig. 35) and also from the comparison of the number of towers (Fig. 36, tower Et>1.0 GeV). The jet width certainly can be widened by increasing the transverse momenta of particles relative to the jet axis, but before we have a complete study this is not guaranteed to be the correct thing to do. The apparent width difference may affect our analysis through the jet energy correction and the relevant quantity is the jet energy out of the clustering cone. But because the CDF clustering algorithm has a relatively big cone (size 0.7 ), the width difference does not immediately disqualify ISAJET as the generator for the jet energy correction study. We will postpone the comparison of the jet energy (Et) flow to next chapter (Ch.7), where its importance is most appropriately emphasized, and we will see that the energy out of the cone is not too different for the Monte Carlo and real data .

We next observed the most alarming difference between the MC and data, i.e. the EM energy fraction (EMF = EM/TOTAL) of jets (Fig. 37). The mean EMF from data is 0.59 , while that from $M C$ is 0.51 . The EM fraction is an intertwined result of the composition of particles, the hadron showering model in the CEM (interaction length + shower development), and the CEM and CHA responses. Let us assume a very simple-minded model where a fraction $A$ of jet energy is carried by particles (mostly the descendents of $\pi^{0}$ 's) which are completely absorbed in the CEM and the remaining fraction 1-A (mostly charged pions) develops both EM cascades and hadronic cascades which are then shared by the CEM and CHA. Define the following quantities:

$A \equiv$ the fraction of the jet energy carried by particles which shower only electromagnetically, e.g. e and $\gamma$ which will be completely absorbed in the CEM

$=1 / 3$

$h \equiv$ the ratio of the CEM responses to hadronic and electromagnetic 
cascades.

$f \equiv$ the fraction of the charged pion energy going into the electromagnetic cascade in CEM.

$e$ the fraction of the charged pion energy absorbed in the CEM (depending on where the shower starts and how it develops).

$r \equiv$ the ratio of the CEM responses to charged pions and electrons $=f+(1-f) h$

The pulse height PH and the EM energy fraction EMF for unit jet energy, neglecting the nonlinearity effect, are given by

$\mathrm{PH}=A+(1-A)\left[\theta^{\cdot} \cdot r+(1-\theta)\right]$,

$E M F=[A+(1-A) e \cdot r] / P H$.

This is so because CEM was calibrated with $(50 \mathrm{GeV})$ electrons and CHA with $(50 \mathrm{GeV}$ ) charged pions. If $f$ is taken to be 0.4 and $h$ to be 0.4 as in the current CDFSIM, then the response ratio $r$ is equal to $0.64(=0.4$ + $0.6 \times 0.4$ ). To get EMF $=0.51$ (the mean EMF of the Monte Carlo data), we need $e=0.323$, which means $32 \%$ of the charged pion energy is absorbed by the CEM. In summary, the mean values for current CDFSIM are $r=0.64$ (because $f$ $=0.4$ and $h=0.4$ ) and $e=0.32$, and the resulting EMF and pulse height $\mathrm{PH}$ are 0.51 and 0.92 .

The values of $\theta$ and $r$ can be varied to get EMF $=0.59$ as observed in real data. We recall that the current value $h=0.4$ in CDFSIM was determined by S. Bertolucci et al. [40] to fit test beam data, but is surprisingly low compared to the value 0.7 one would expect from Wigmans' data $[41,42]$. With $h=0.7$ and the CDFSIM value $f=0.4$, we would have $r=$ 0.82 . The following table gives the results of varying $e$ and $r$ :

$\begin{array}{llllllll}\text { set } A & e & r & \text { EMF } & \text { PH } & \Delta P H(x) & \text { comments } \\ 1 & 1 / 3 & 0.323 & 0.64 & 0.510 & 0.923 & \equiv 0 & \text { CDFSIM } r \\ 2 & 1 / 3 & 0.323 & 0.82 & 0.530 & 0.961 & +4.1 & \text { Wigmans } r \\ 3 & 1 / 3 & 0.450 & 0.64 & 0.589 & 0.890 & -3.6 & \text { correct EMF, CDFSIM } r \\ 4 & 1 / 3 & 0.410 & 0.82 & 0.586 & 0.950 & +3.0 & \text { correct EMF, Wigmans } r \\ 5 & 0.43 & 0.323 & 0.64 & 0.587 & 0.934 & +1.2 & \text { correct EMF, CDFSIM } r\end{array}$

the $\triangle P H$ for high EMF (given by high $e$ ):
$\begin{array}{lll}6 & 1 / 3 & 0.800\end{array}$
0.64
0.835
$0.808 \equiv 0$
high EMF, CDFSIM $r$
$7 \quad 1 / 3$
0.900
0.64
0.915
$0.784+3.0$
8\% higher EMF, CDFSIM $r$ 
This table roughly tells us that the calorimeter energy given by the simulation can be incorrect up to $4 \%$ due to incorrect values of $e$ and $r$. In set 5 we varied $A$, the particle composition of jet, to give the correct $E M F$, and found the effect on $\mathrm{PH}$ is smaller (1\% level). The particle composition of ISAJET seems to be right when one compares the ratio of the charged track energy to calorimeter energy [43]. The single particle response (CEM+CHA) in Monte Carlo has been tuned to the minimum bias and the test beam data [44] (see Fig. 97 in Appendix E). If ISAJET (charged/total $=0.65$ ) is right, the correct values of $e$ and $r$ may be a compromise between set 3 and set 4. Again the difference in EMF shouldn't affect our energy correction.

Other comparisons show reasonable agreement between data and Monte Carlo. The mass of the jet (calculated from tower energy and momenta assuming massless particles, Fig. 38) is slightly low in the Monte Carlo, a fact related to our previous observation that ISAJET gives narrower jets. The $\Delta \phi$ between the leading two jets (Fig. 39) and their Pt ratio (Fig. 40) for dijet events are in excellent agreement. The Pt (transverse to the $p$ and $\vec{p}$ beams, Fig. $41(a)$ ) and $P_{l}$ (parallel to the beams, Fig. 41(b)) are slightly high in the MC. The agreement in the ratio of the third to the second highest jet Pt's (Pt3/Pt2, Fig. 43) for dijet events is better than we have any right to expect because the simulation for the gas calorimeters is not tuned and the detector noise in these systems is not simulated. Finally the magnitude of missing Pt (vector Pt sum of all jets, Fig. 44) is slightly lower in the MC. 
Table 4 Comparison of clustering algorithms (from CDF note 605 )

\begin{tabular}{|c|c|c|c|c|}
\hline & CLUST2 & CLUST3 & CLUST4 & JETCLU \\
\hline CPU sec/event (VAX8650) & 0.075 & 0.093 & 0.141 & 0.063 \\
\hline Embedded clusters & $3 \%$ & $0 \%$ & $O \%$ & $0 \%$ \\
\hline Large clusters & $1 \%$ & $0 \%$ & $0 \%$ & $0 \%$ \\
\hline Mean full $\eta$ width & 0.73 & 0.82 & 0.78 & 0.95 \\
\hline Mean full $\phi$ width & 1.21 & 1.17 & 1.25 & 1.26 \\
\hline Pt resolution (GeV) & 13.4 & 13.0 & 11.3 & 11.4 \\
\hline \multicolumn{5}{|l|}{ Event Mixing: } \\
\hline Et (before)-Et (after) (GeV) & 0.96 & 0.72 & 0.90 & 0.43 \\
\hline merging distance & 0.62 & 0.64 & 0.71 & 0.79 \\
\hline merging sharpness & 0.19 & 0.14 & 0.22 & 0.15 \\
\hline merging dist. (Et>25GeV) & 0.77 & 0.67 & 0.65 & 0.81 \\
\hline $\begin{array}{c}\text { merging sharpness } \\
\text { (Et)25GeV) }\end{array}$ & 0.17 & 0.07 & 0.33 & 0.12 \\
\hline
\end{tabular}


Fig. 30 Comparison of jets as found by the JETCLU and JCLST4 algorithms:

a) the difference in jet $\eta$ 's;

b) the difference in jet $\phi$ 's;

c) the difference in jet Et's;

d) the difference in the numbers of towers in jets.

All jets have Et $>30 \mathrm{GeV}$ and $|\eta|<0.7$ as found by JETCLU. 

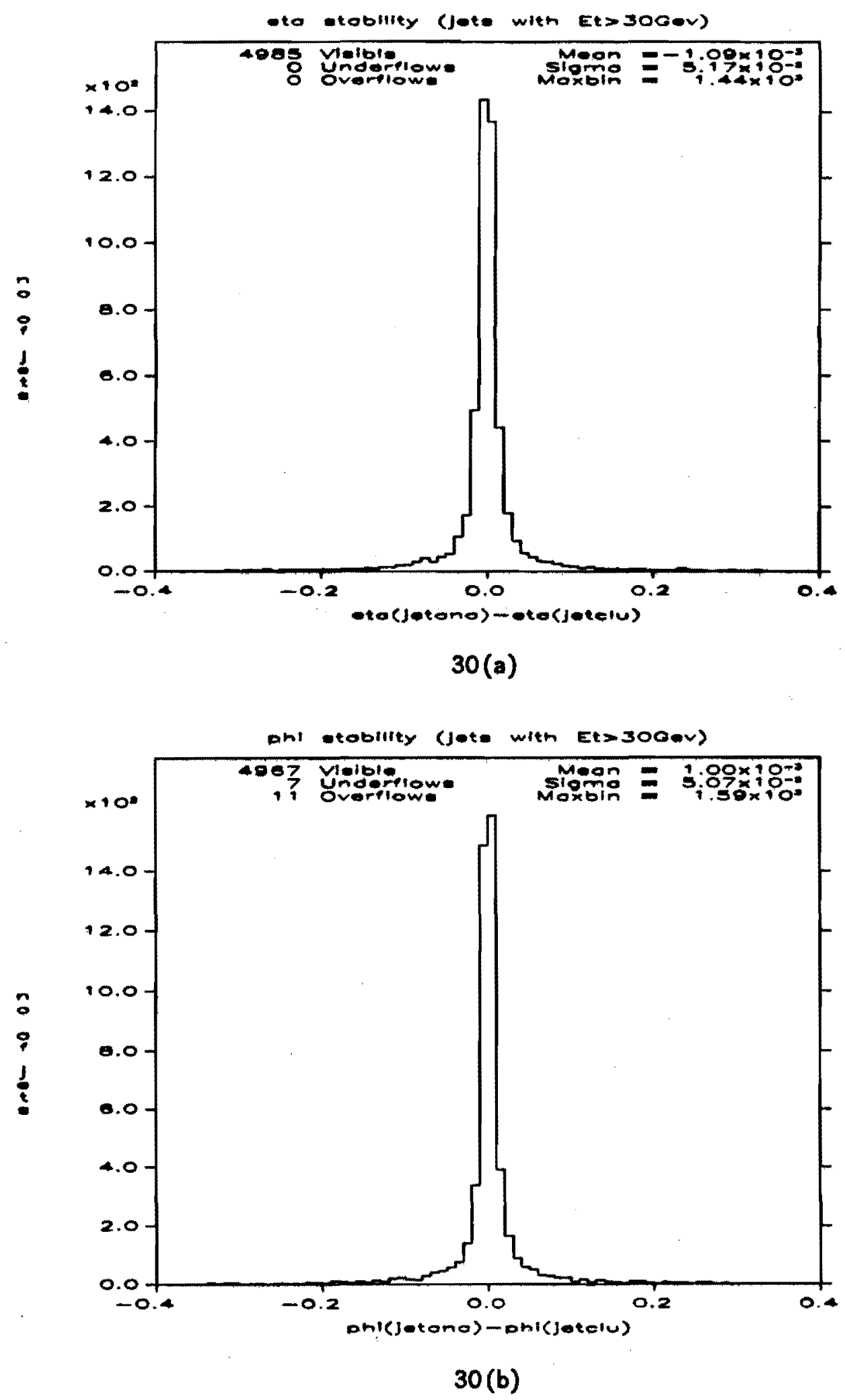

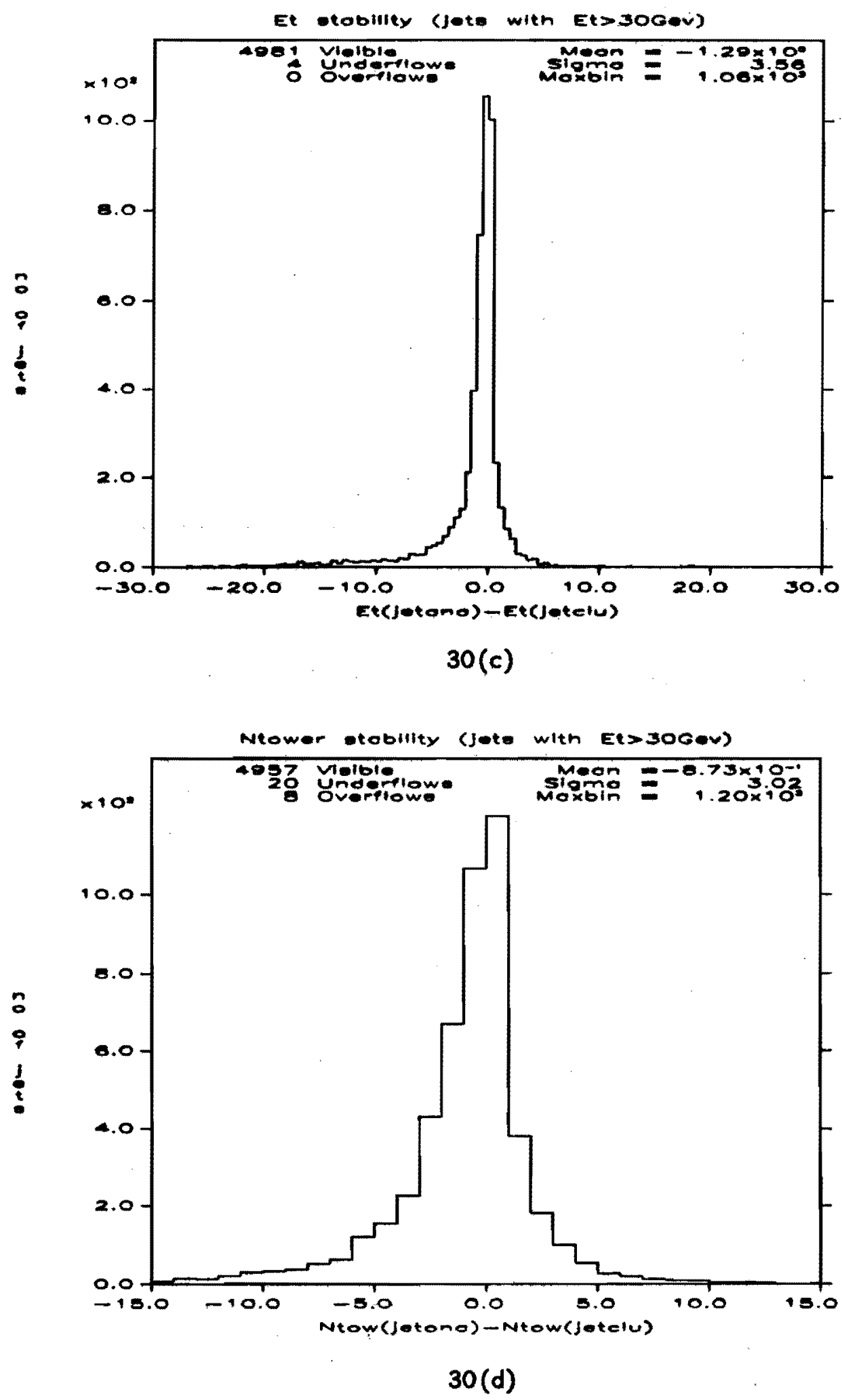


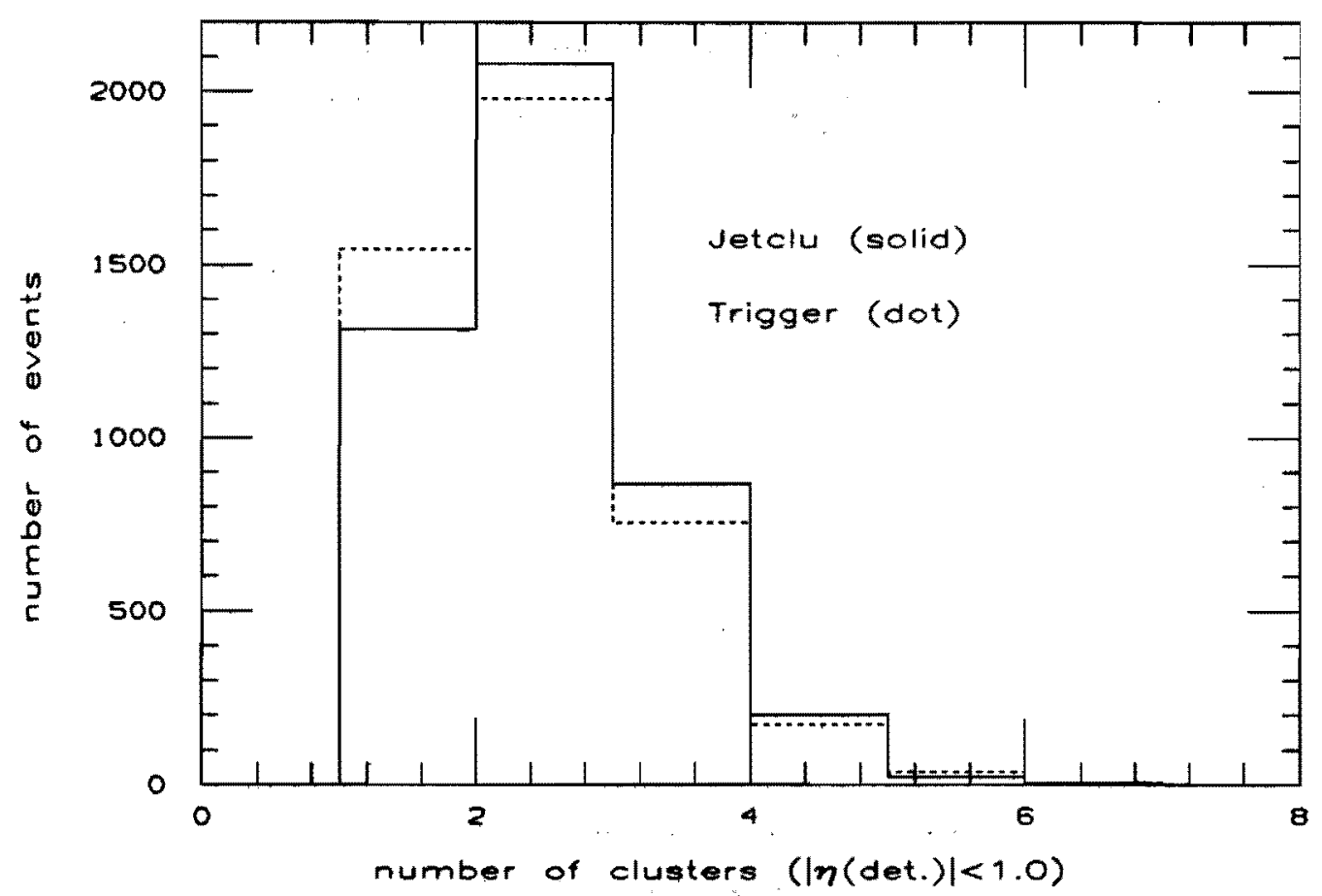

Fig. $31(a)$ The number of clusters found by the Trigger and Jetclu in the central calorimeters.

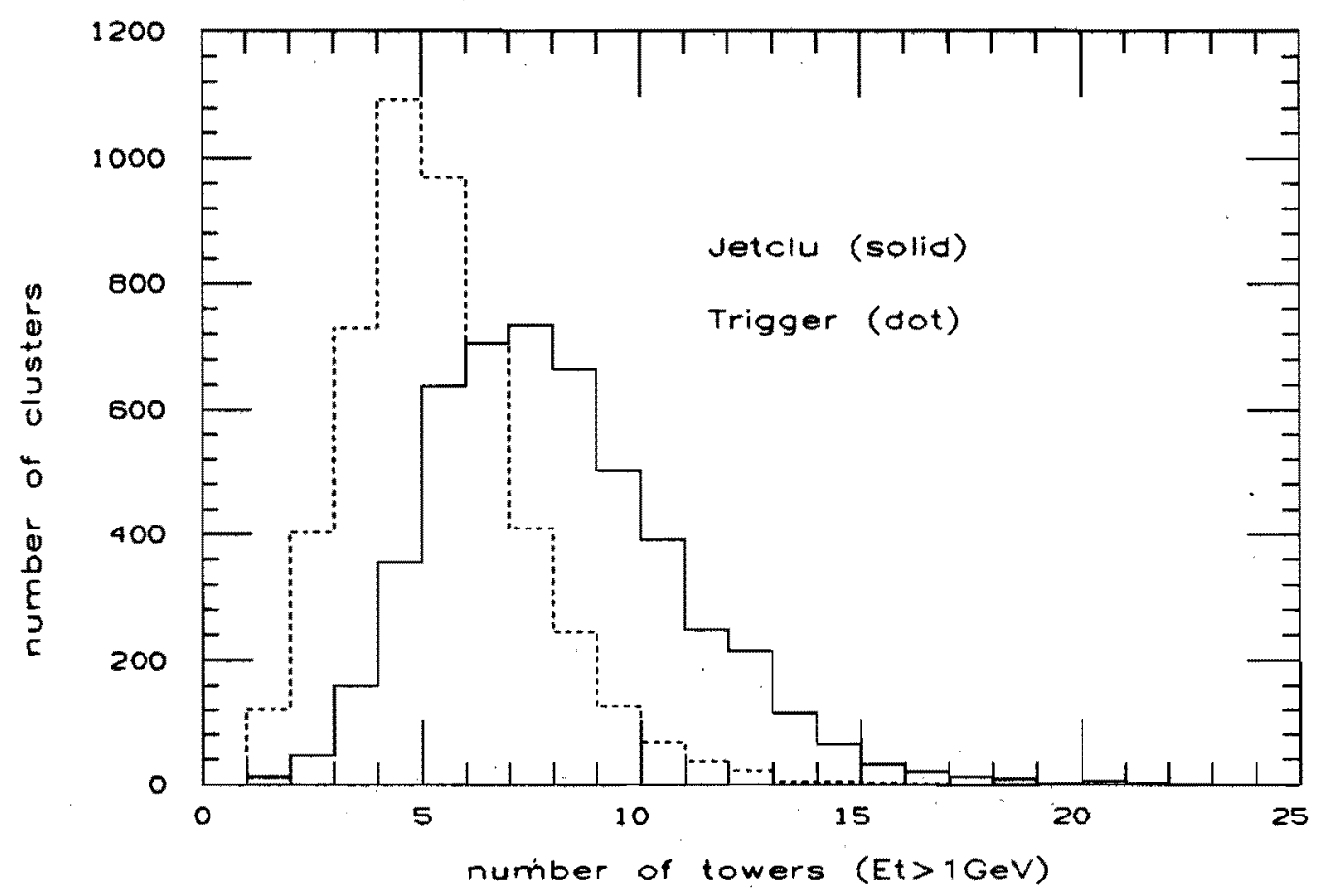

Fig. $31(b)$ Number of towers in jets $(|\eta|<0.7$, Et $>30 \mathrm{GeV})$ and the matched Trigger clusters. 
Fig. 32 Comparison of clusters found by the JETCLU and the Trigger Level-2 cluster finding algorithms:

a) the difference in cluster $\eta$ 's;

b) the difference in cluster $\phi$ 's;

c) the number of Trigger clusters within a radius of 0.7 on $\eta-\phi$ space from a JETCLU cluster;

d) the fraction of the Et sum of the Trigger clusters within 0.7 from a JETCLU cluster found to be contained in the highest Et Trigger cluster. 


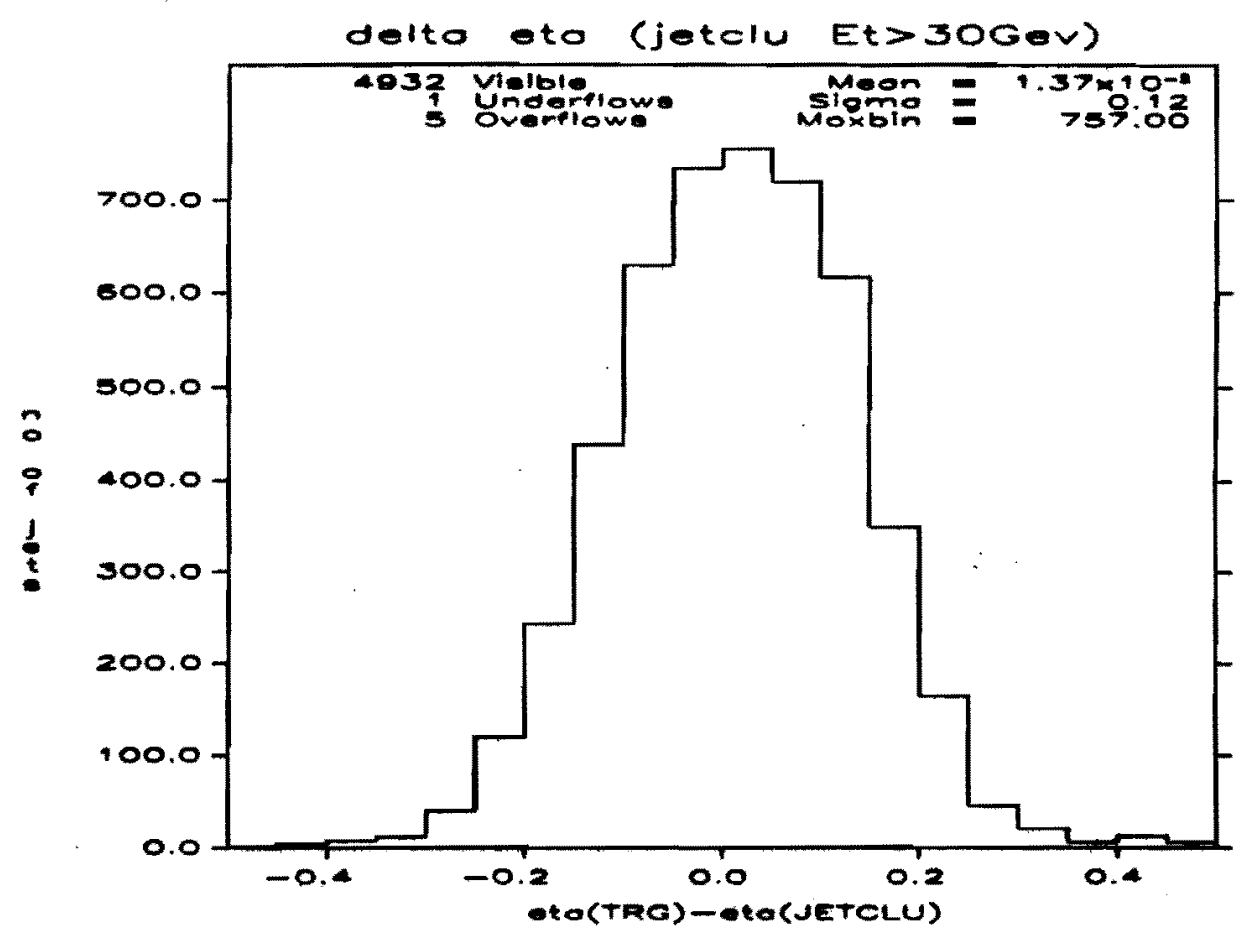

32(a)

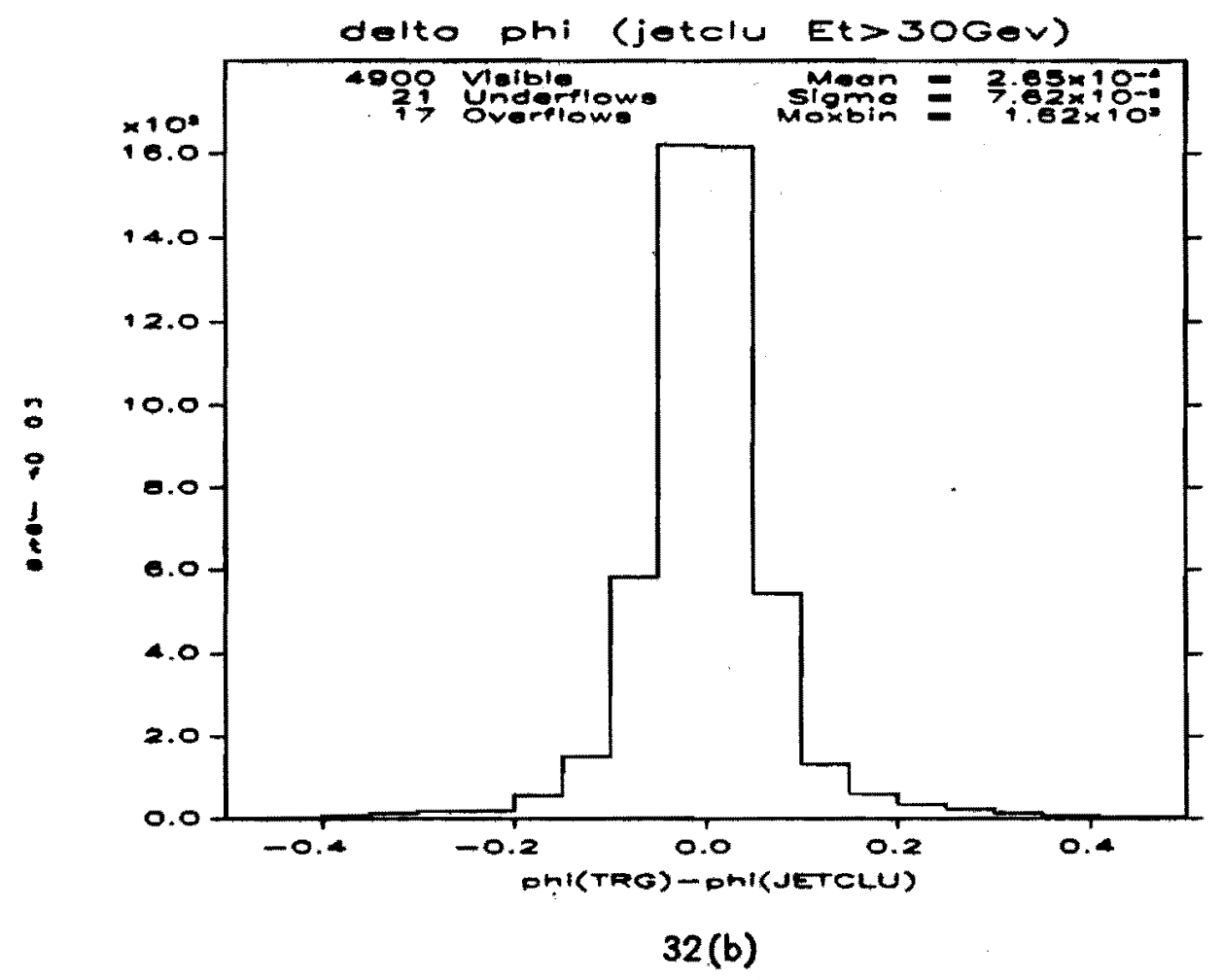



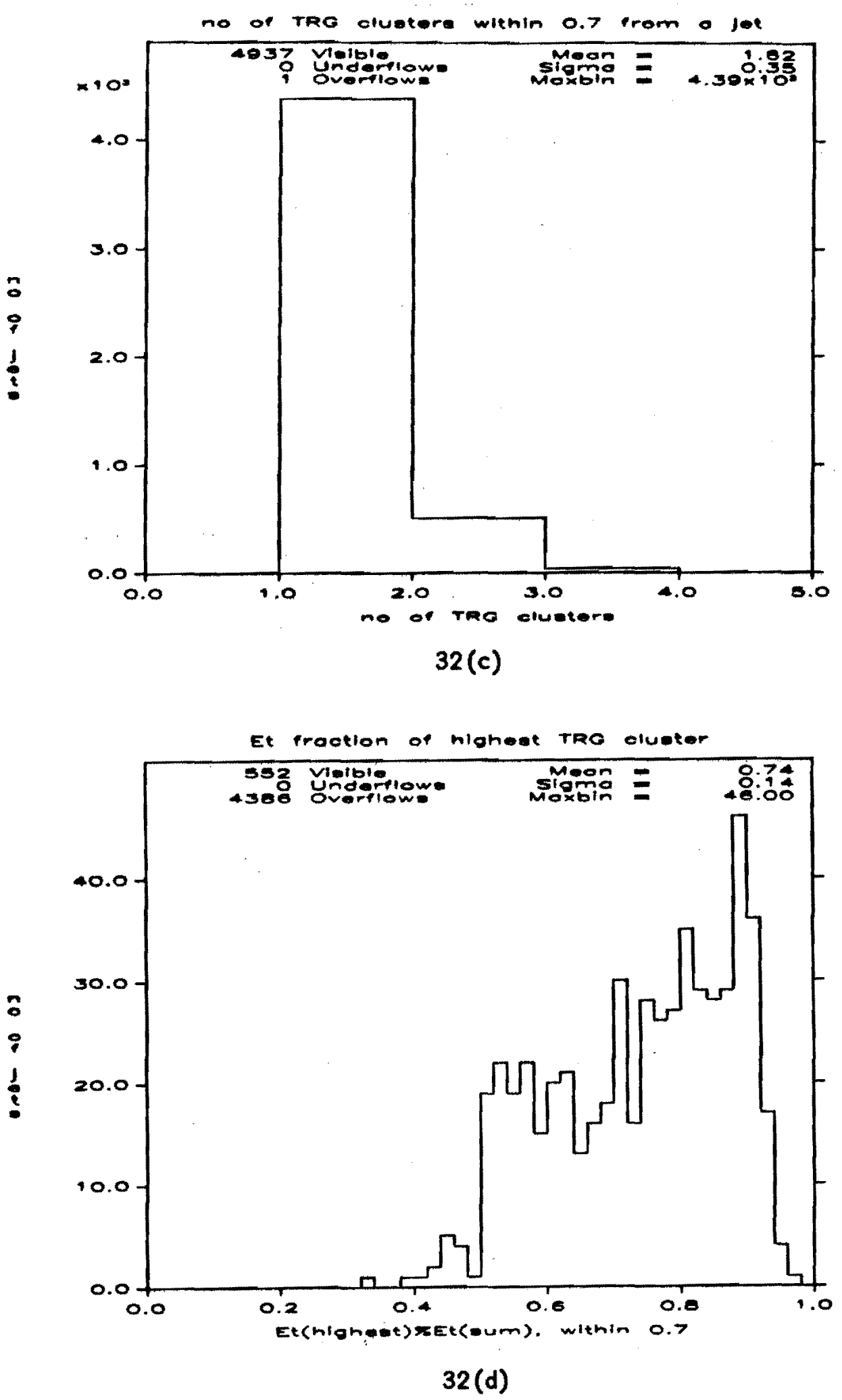


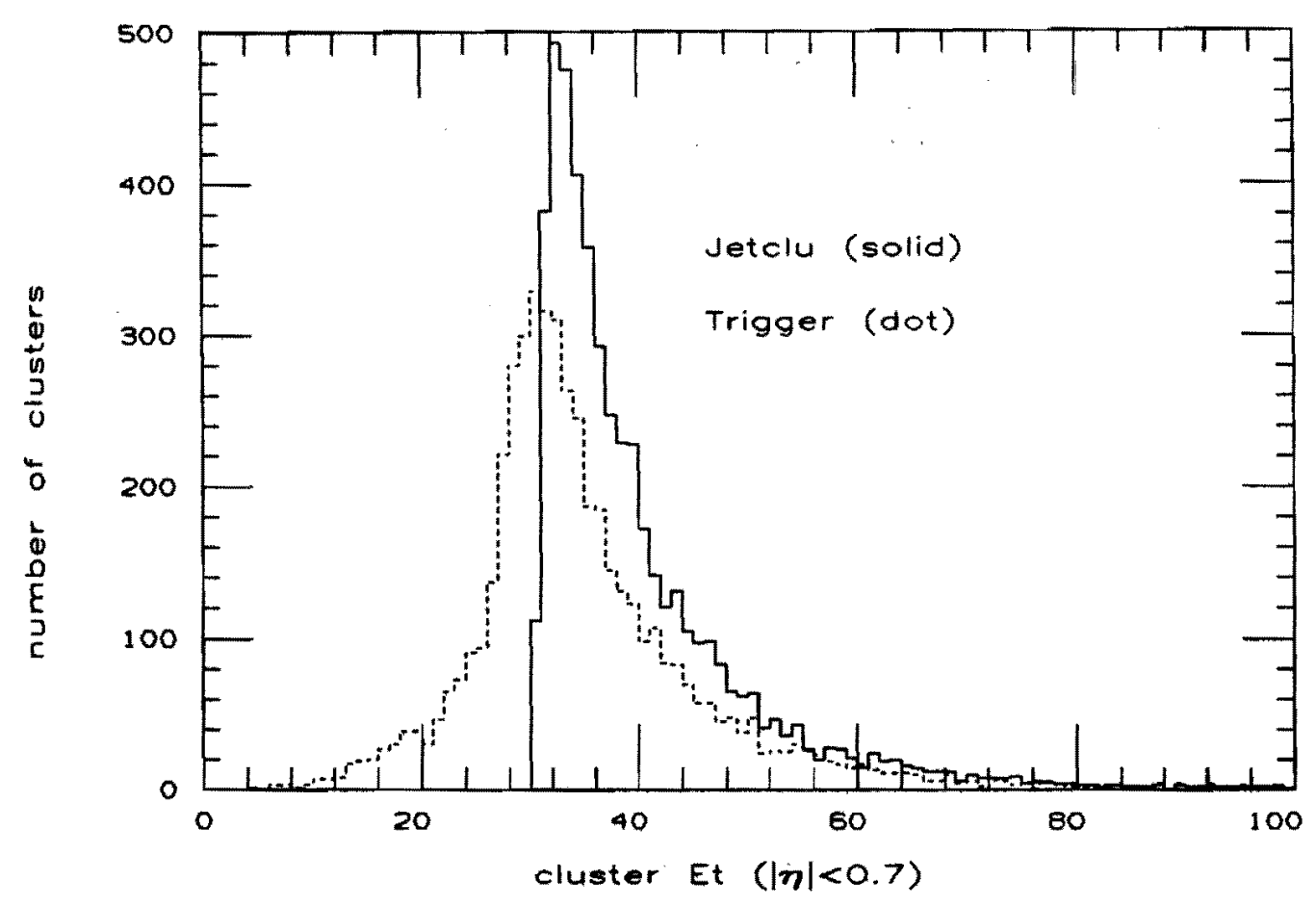

Fig. $33(a)$ Jetclu and Trigger cluster Et distributions

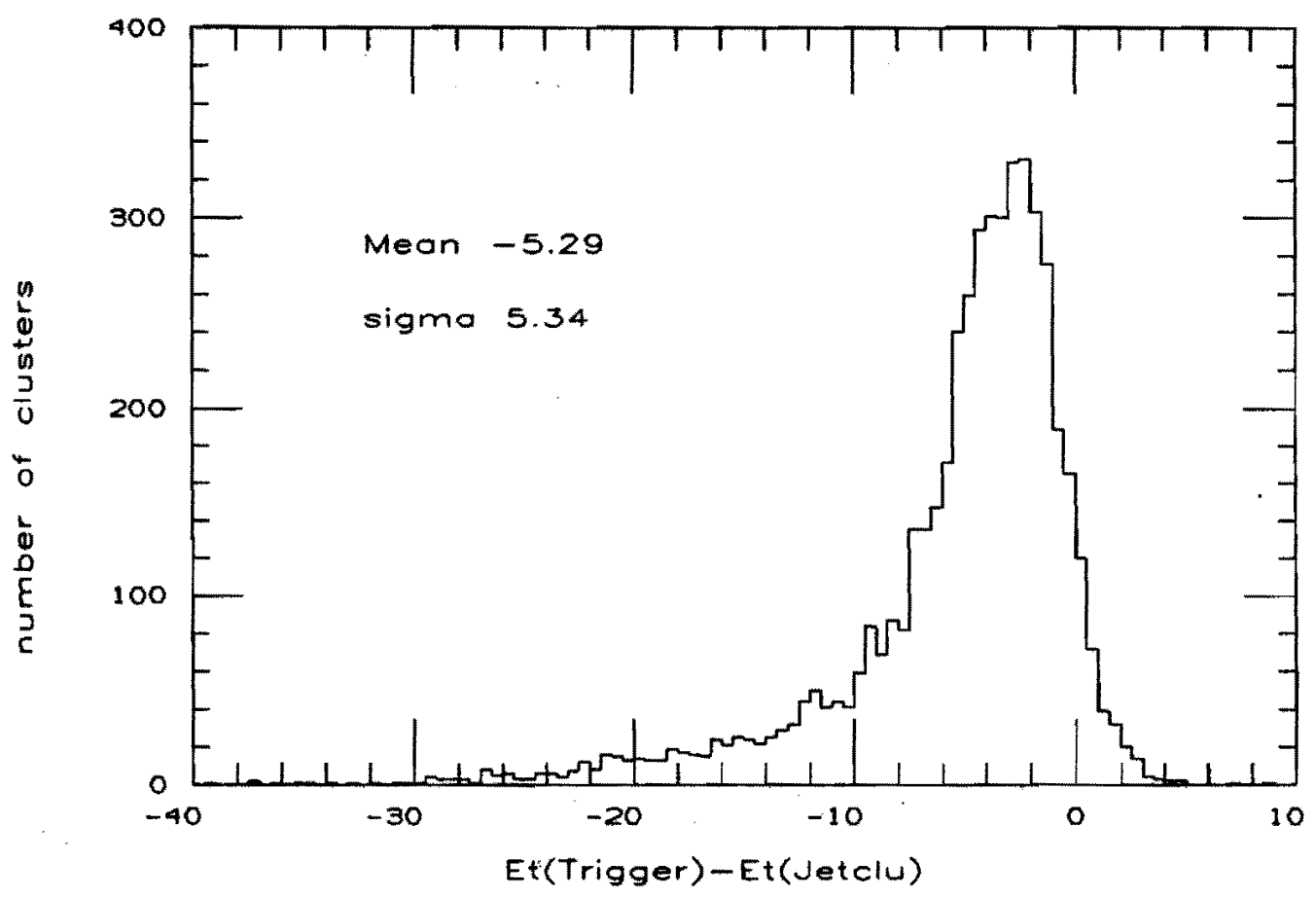

Fig. $33(b) \quad \Delta E t$ Between Jetclu and Trigger clusters (Jetclu Et $>30 \mathrm{GeV}$ ) 
Fig. 34 The efficiency of the Trigger Level-2 algorithm.

a) the Trigger cluster Et distribution for jets with Et>30 GeV as found by JETCLU;

b) the fraction of the events in the $\mathrm{Et}>30 \mathrm{GeV}$ sample that pass certain Trigger cluster Et threshold;

c) the Trigger cluster Et distribution for jets with Et>45 GeV as found by JETCLU;

d) the fraction of the events in the Et $>45 \mathrm{GeV}$ sample that pass certain Trigger cluster Et threshold. 


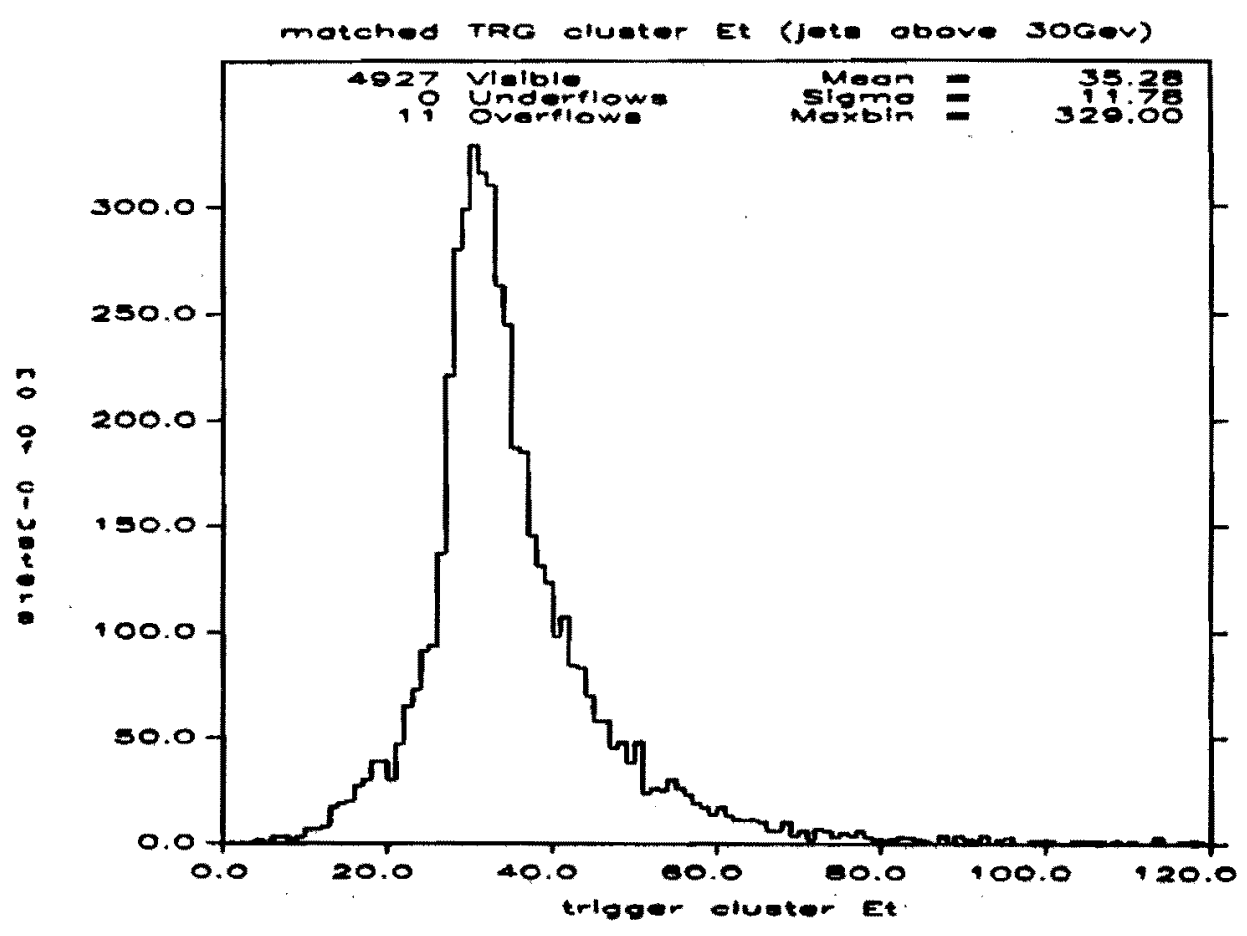

34 (a)

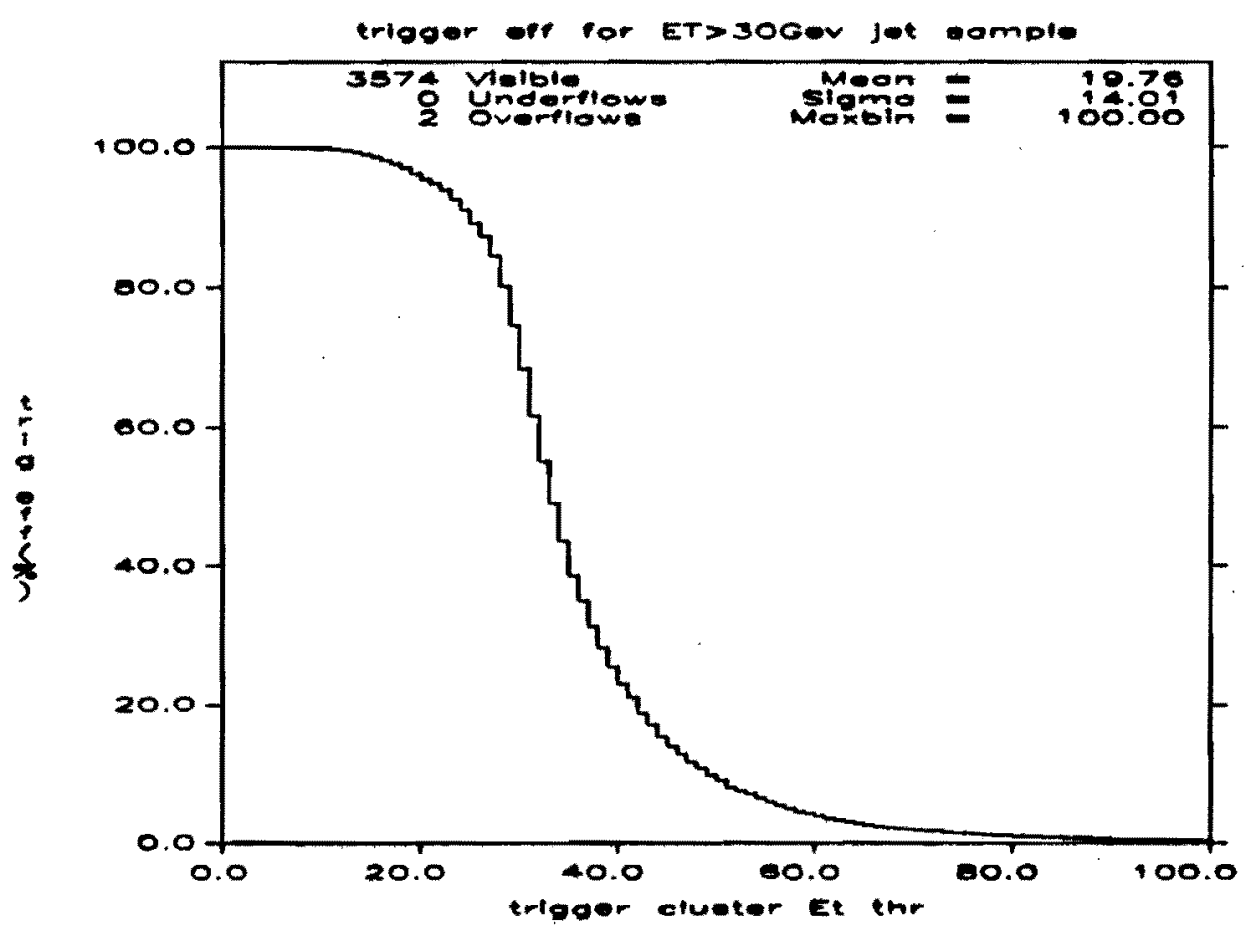

34 (b) 


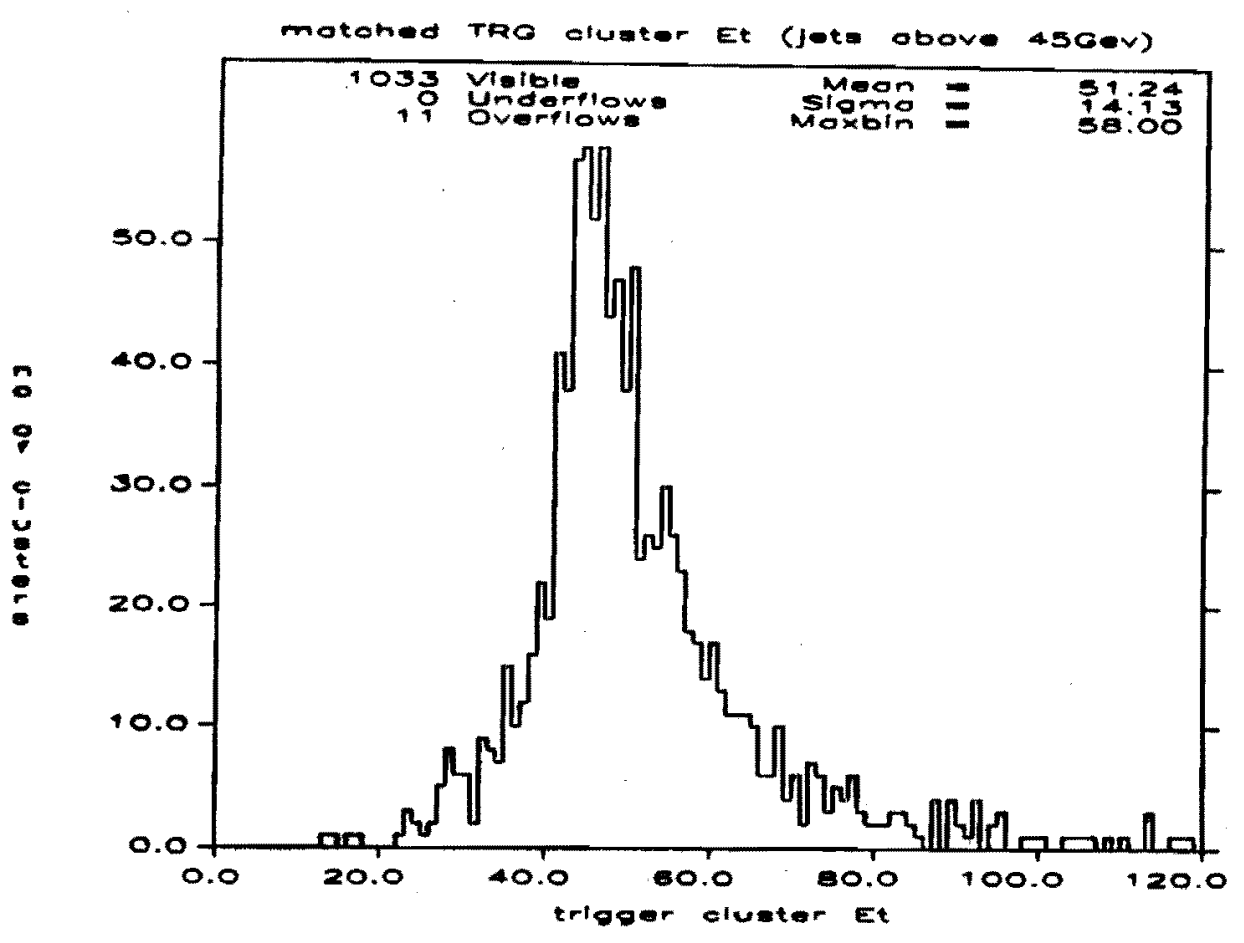

34 (c)

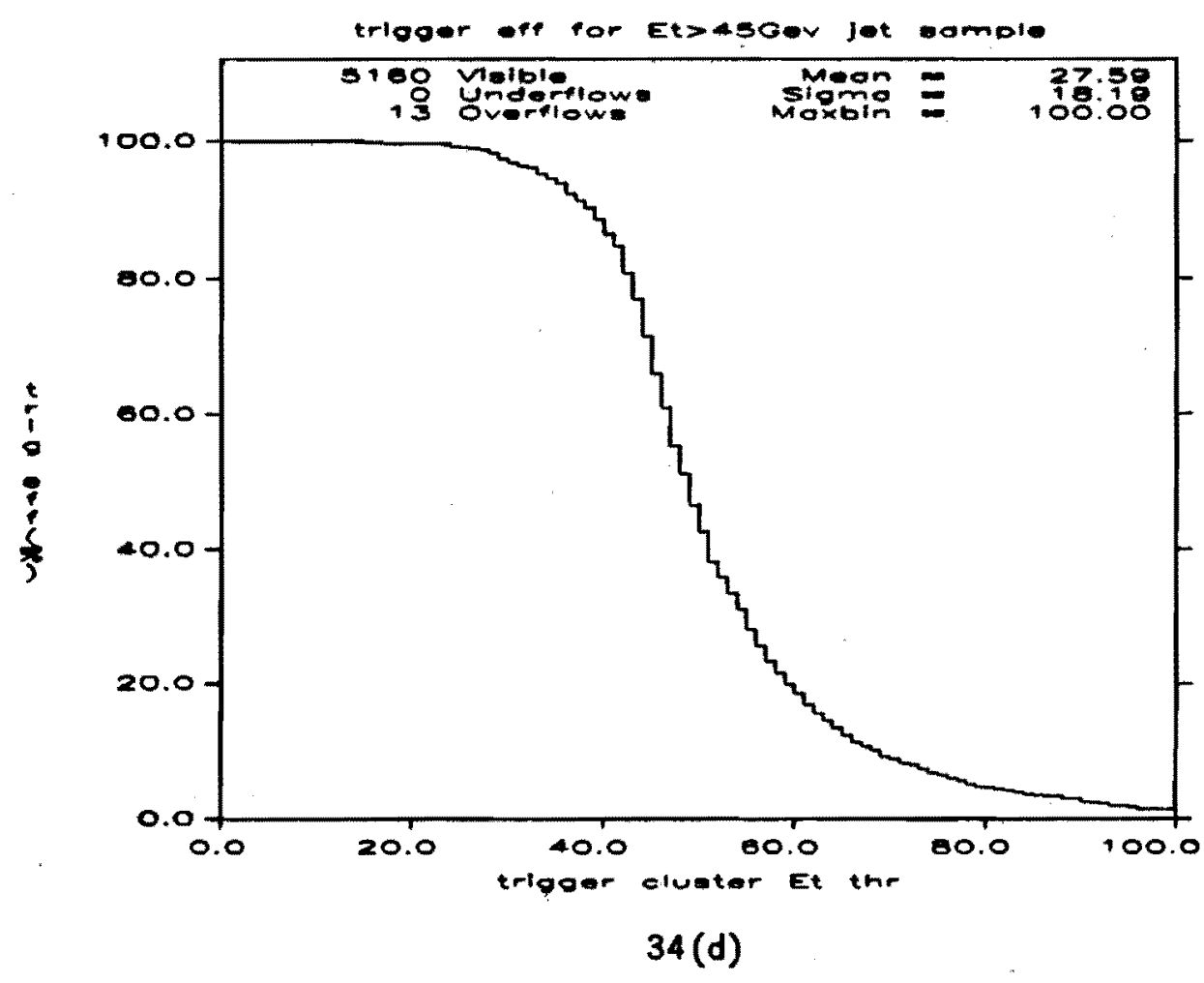




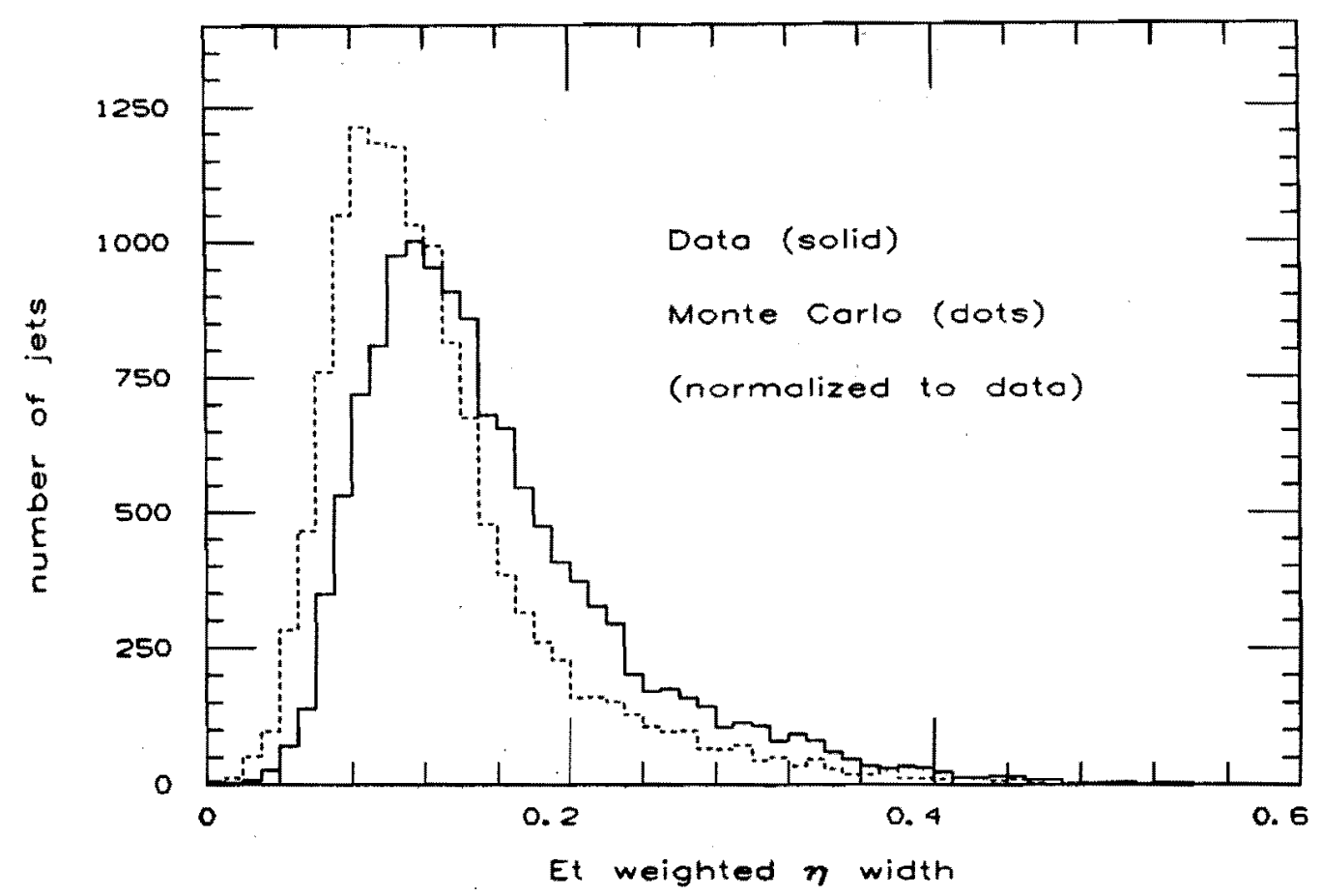

Fig. $35(a)$ Et weighted $\boldsymbol{\eta}$ widths for jets with Et>30GeV Data: $m$ 0.16. $\sigma 0.00744$; MC: $m$ 0.13, o 0.00638

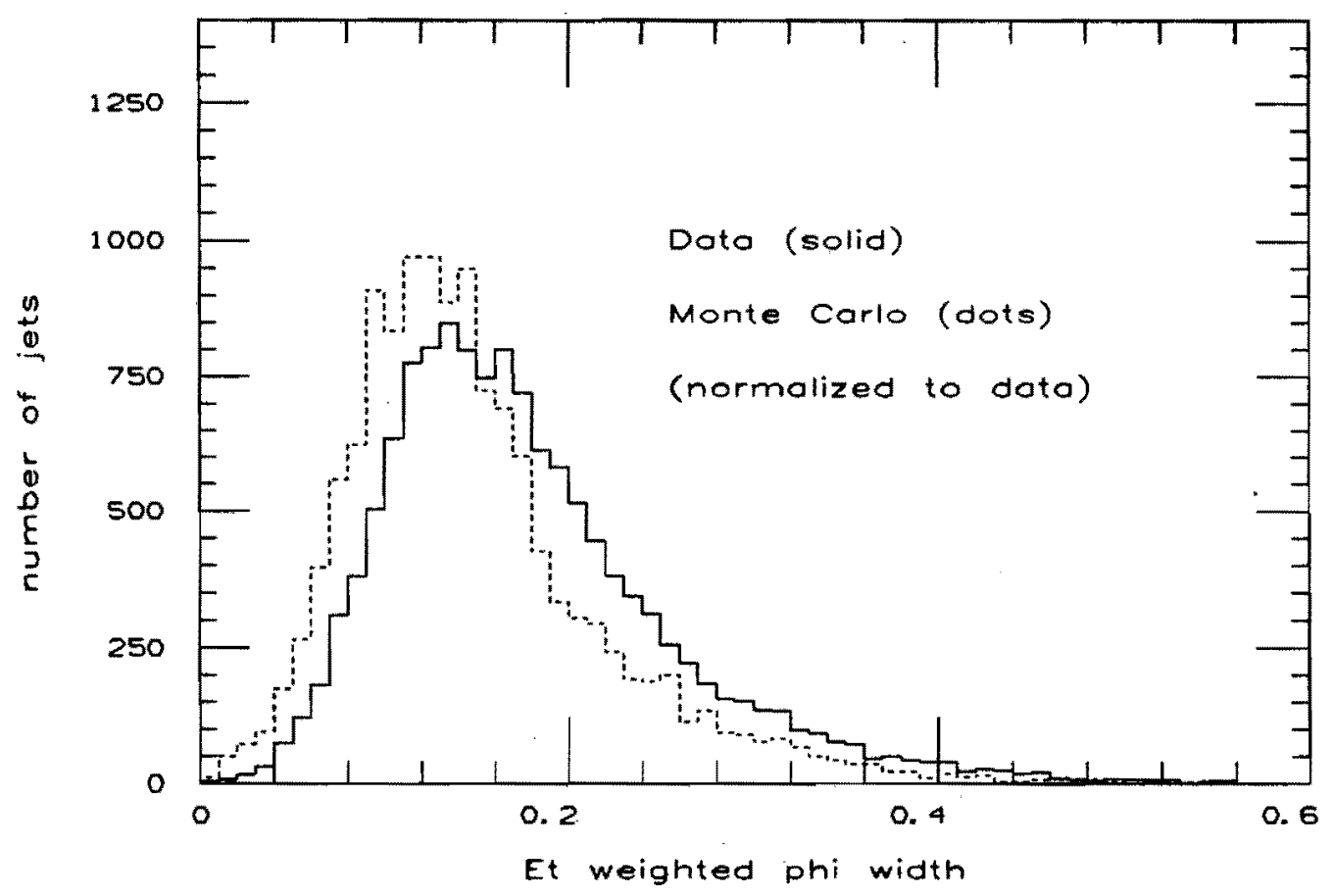

Fig. 35(b) Et weighted $\varphi$ widths for jets with Et>30GeV Data: $m$ 0.15. o 0.00702: MC: $m$ 0.18, $\sigma 0.00785$ 


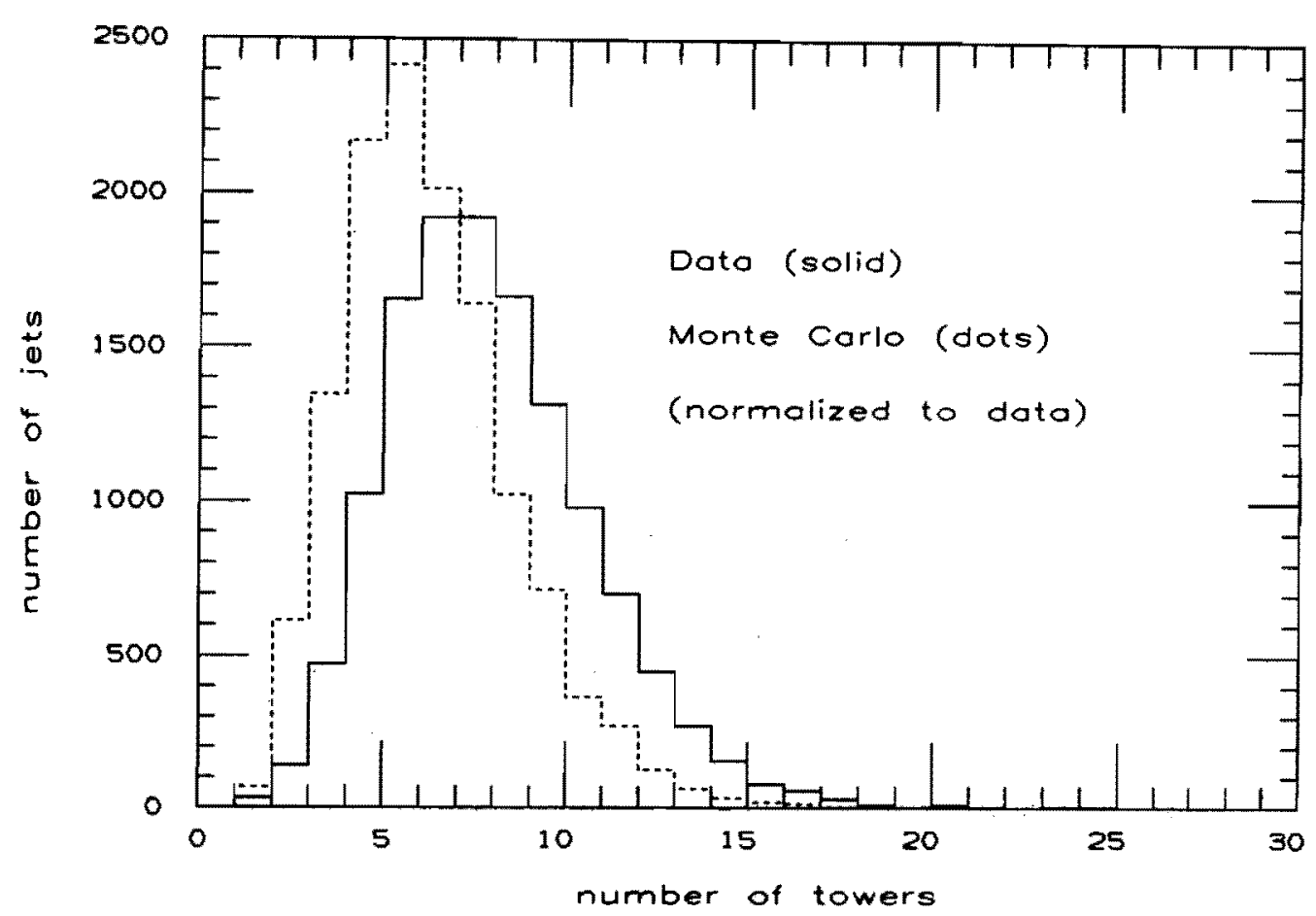

Fig. $36(0)$ Number of towers for jets with Et $>30 \mathrm{GeV}$ Data: $m=7.96, \sigma=2.82$; MC: $m=6.26, \sigma=2.36$

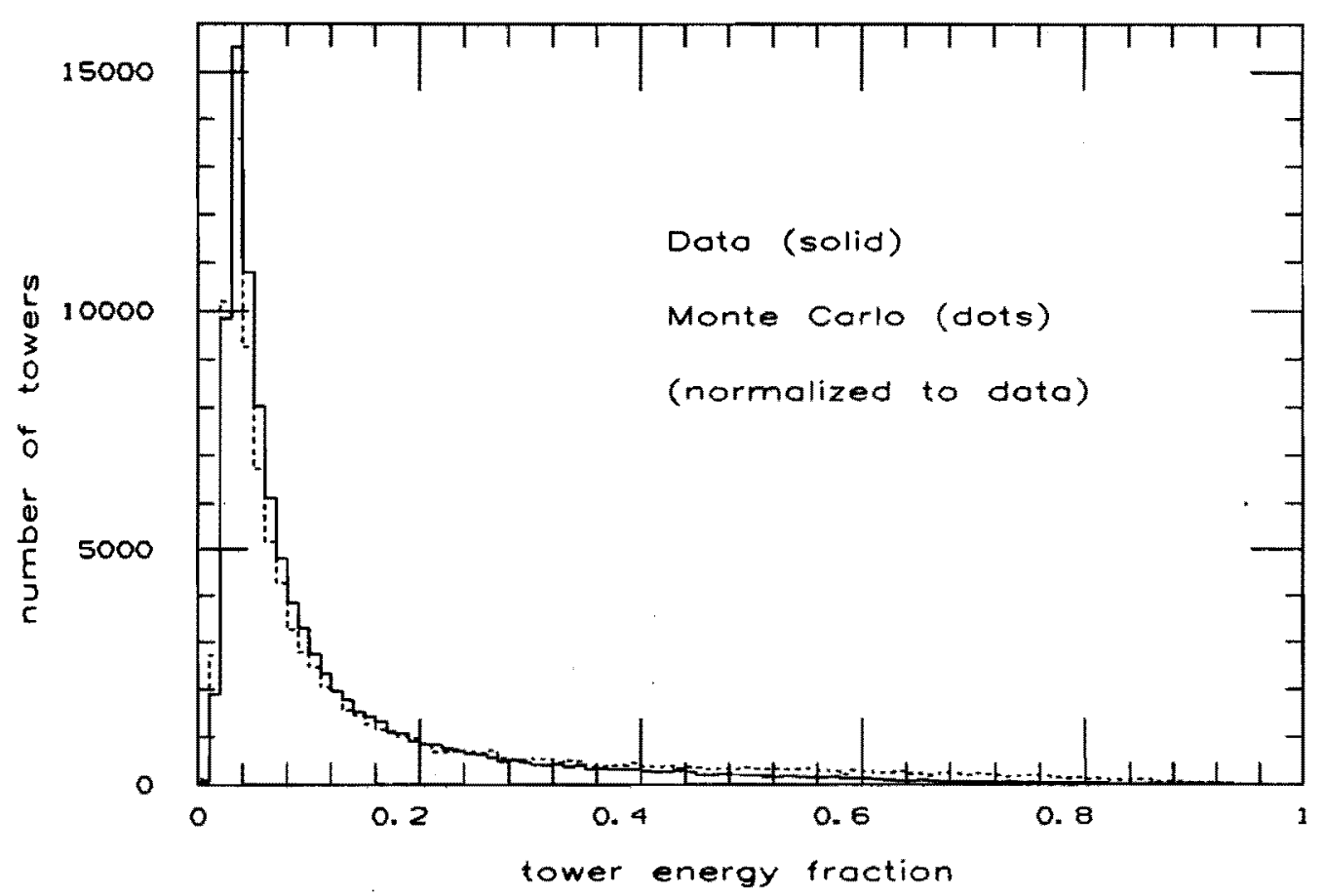

Fig. 36(b) Tower energy fraction $E$ (tower)/E(jet) 


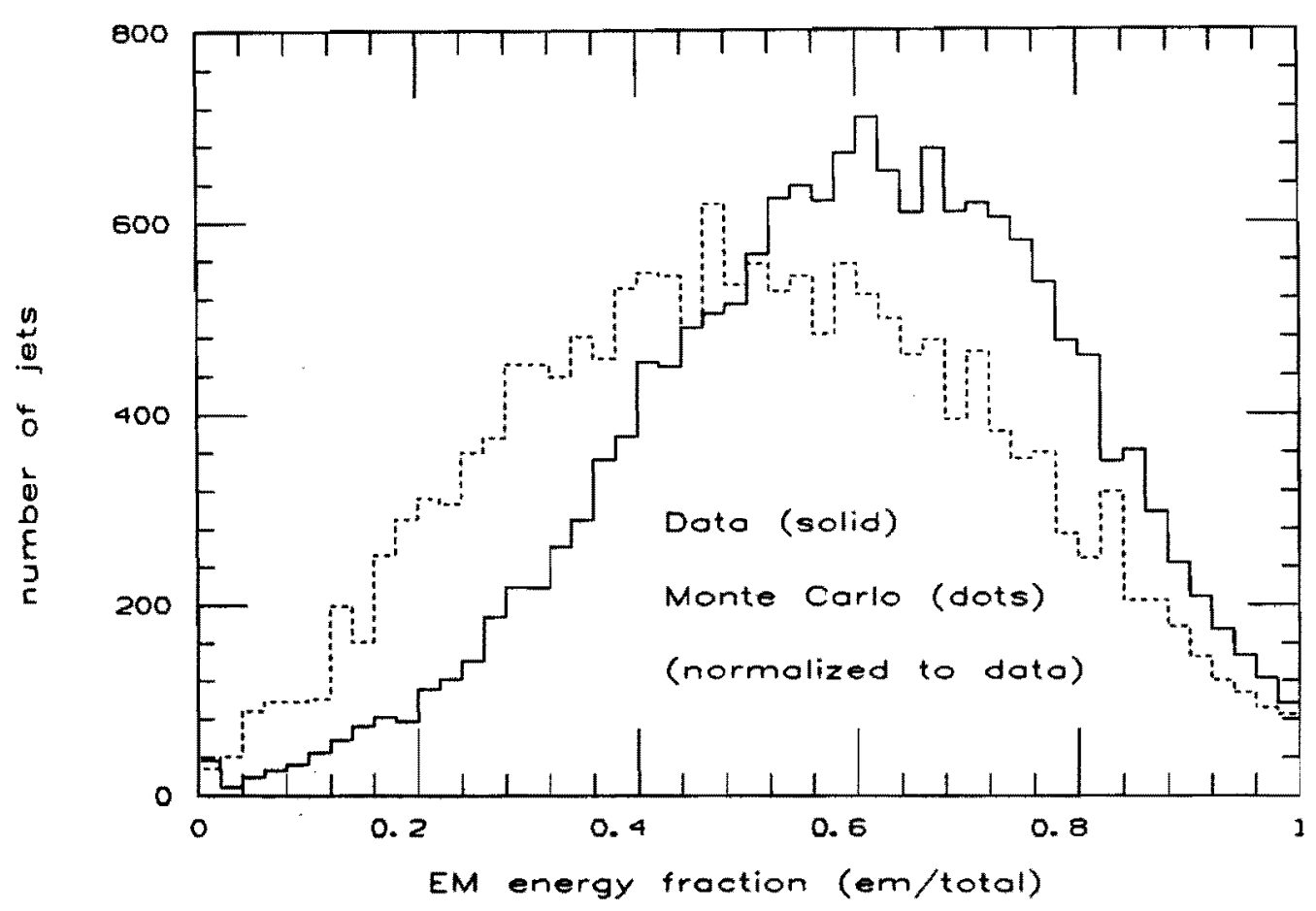

Fig. 37 EM energy fraction for jets with Et>30GeV. Data: $m=0.59, \sigma=0.19$; MC: $m=0.51, \sigma=0.22$

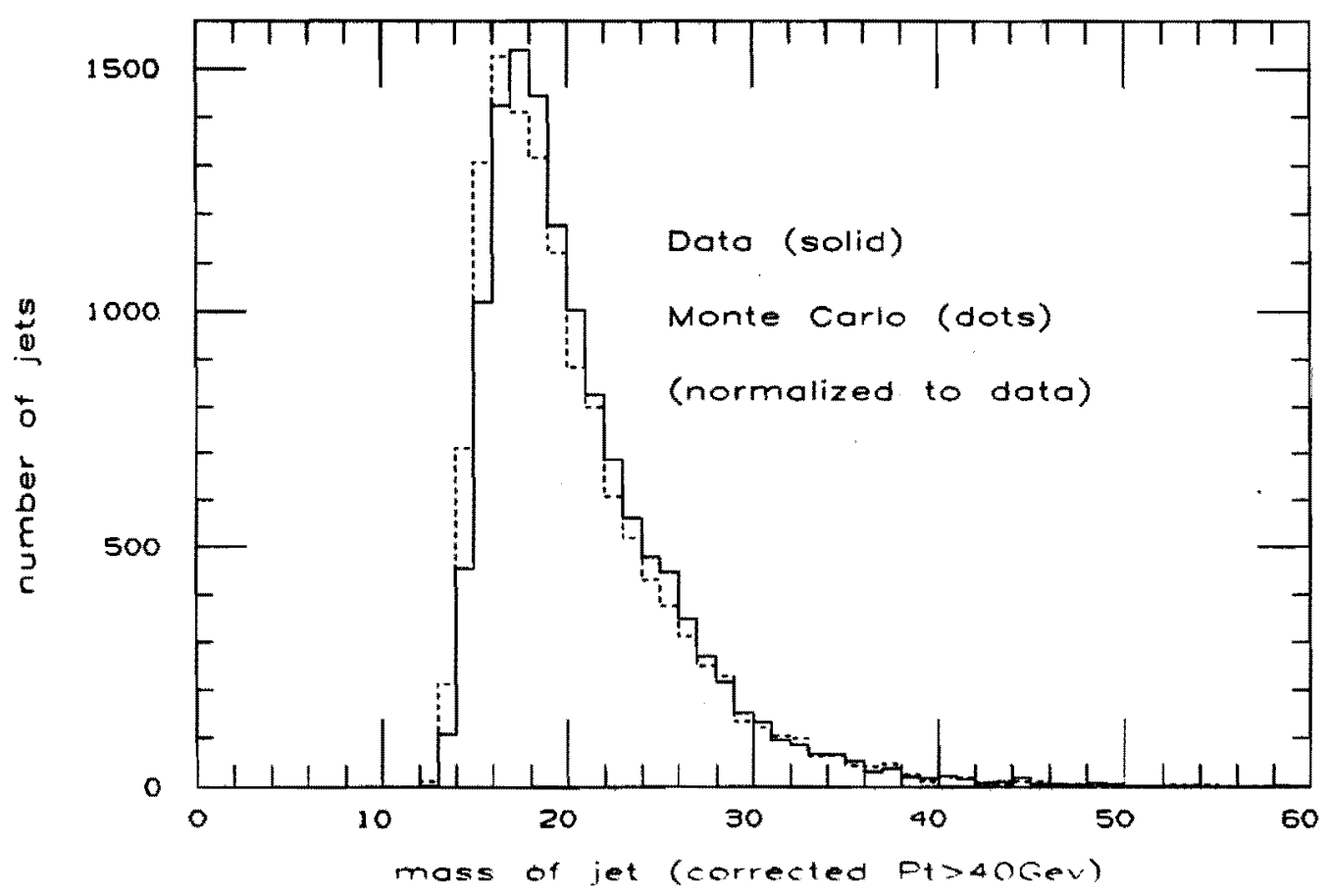

Fig. 38 Masses of jets with corrected Pt>4OGeV. 


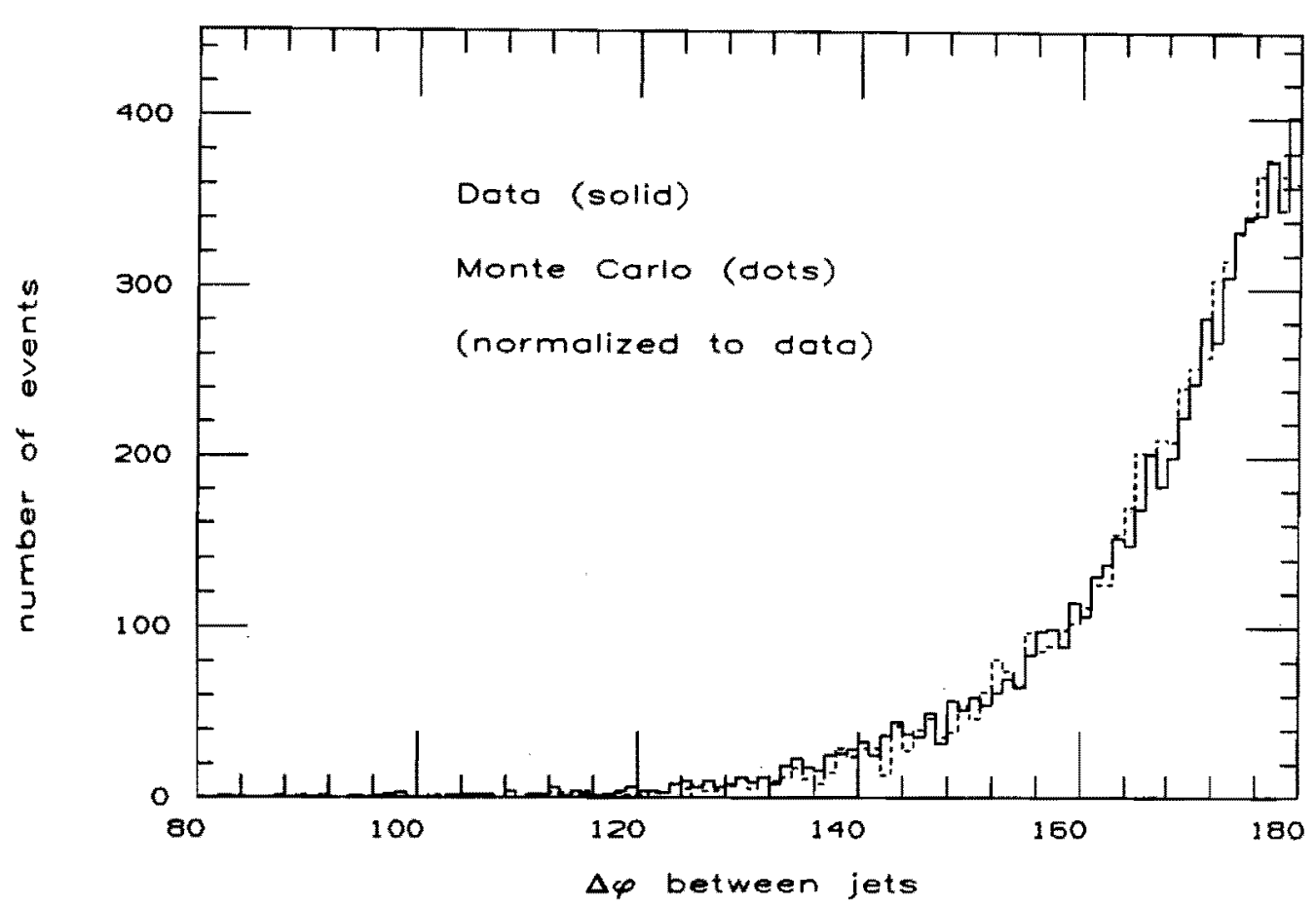

Fig. $39 \Delta \varphi$ between the two leading central jets

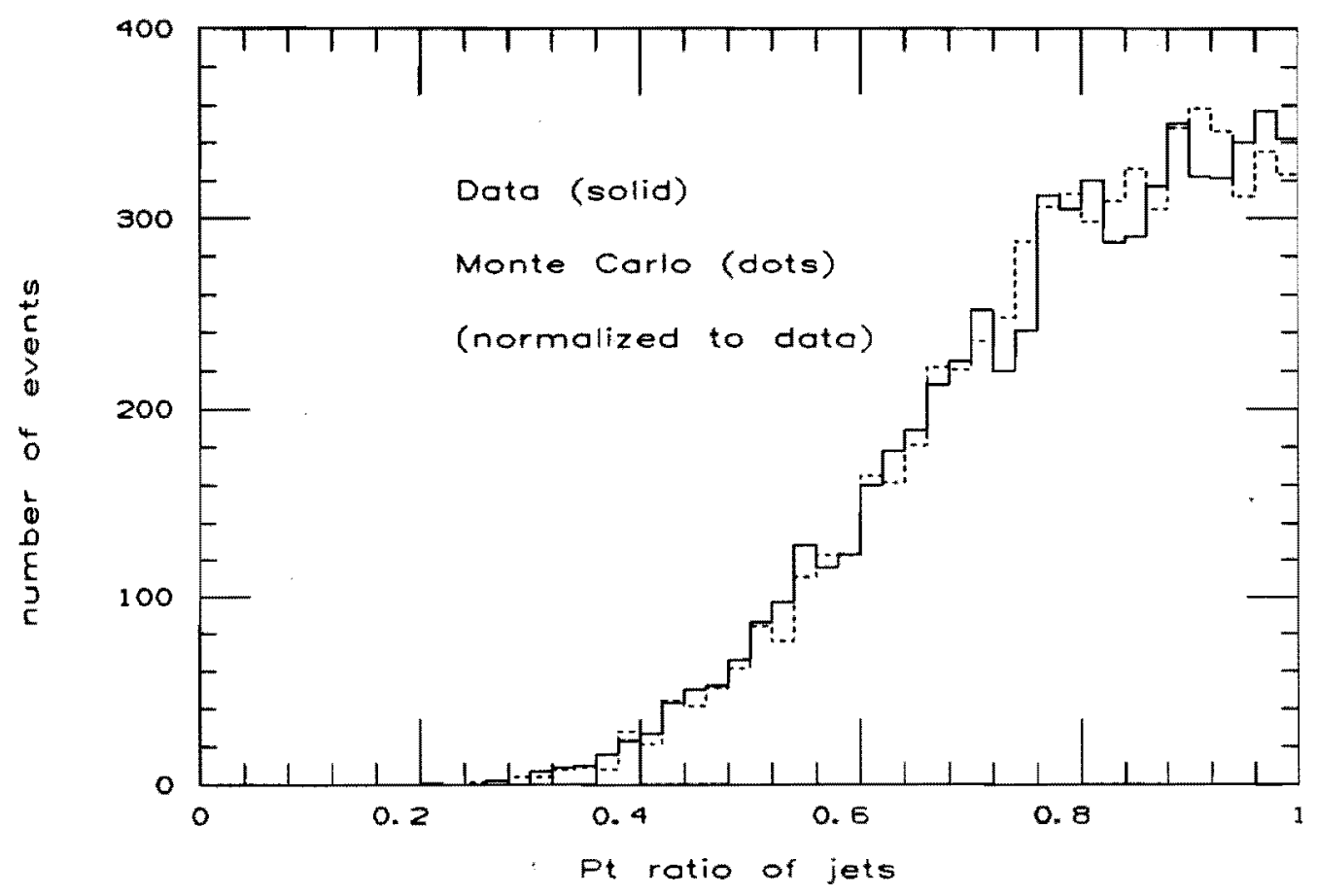

Fig. 40 Pt ratios of the two leading central jets 


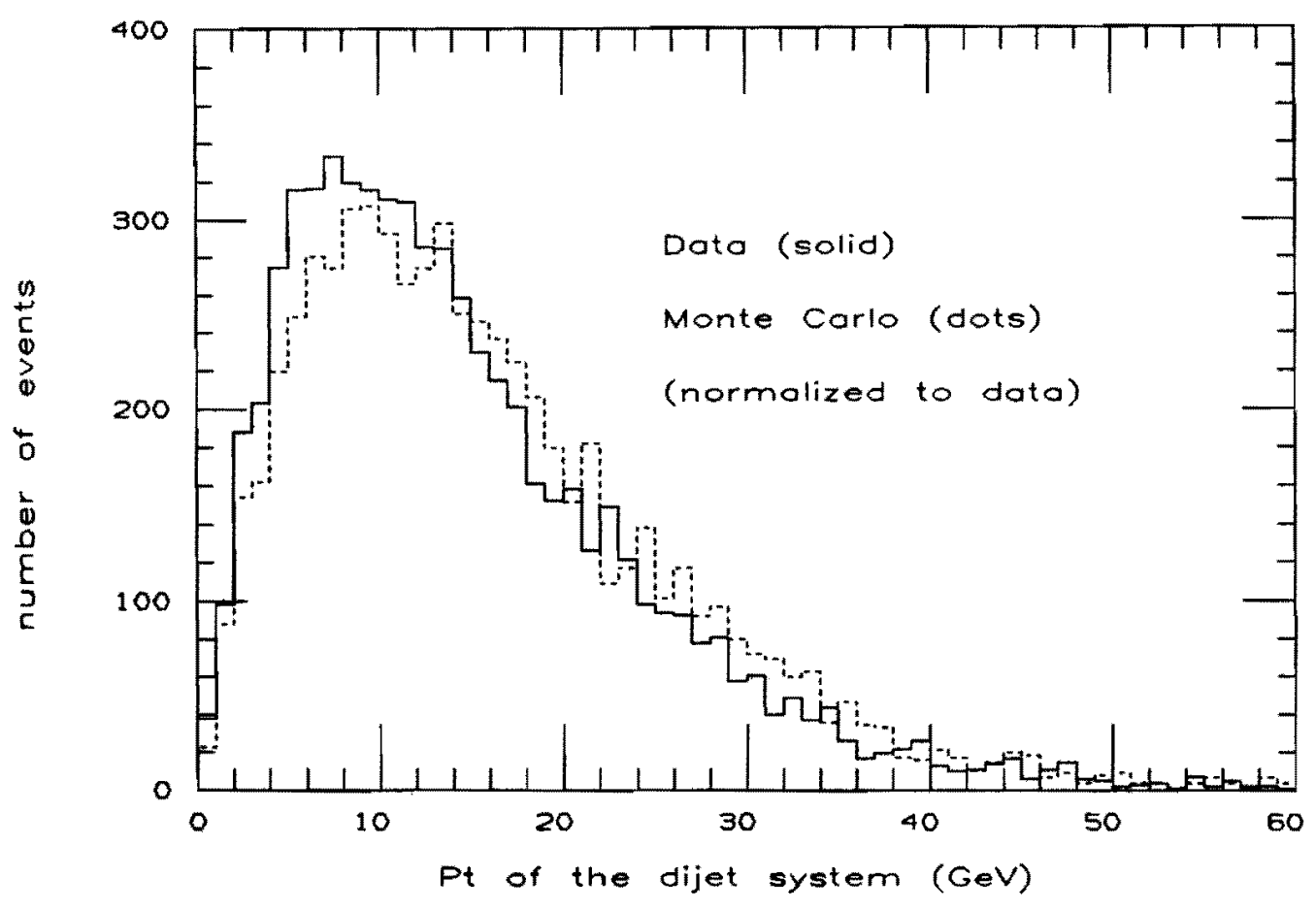

Fig. $41(a) \quad P t$ of the centrol dijet system. Doto: $m=14.61, \quad \sigma=9.63 ;$ MC: $m=15.96, \quad \sigma=10.07$.

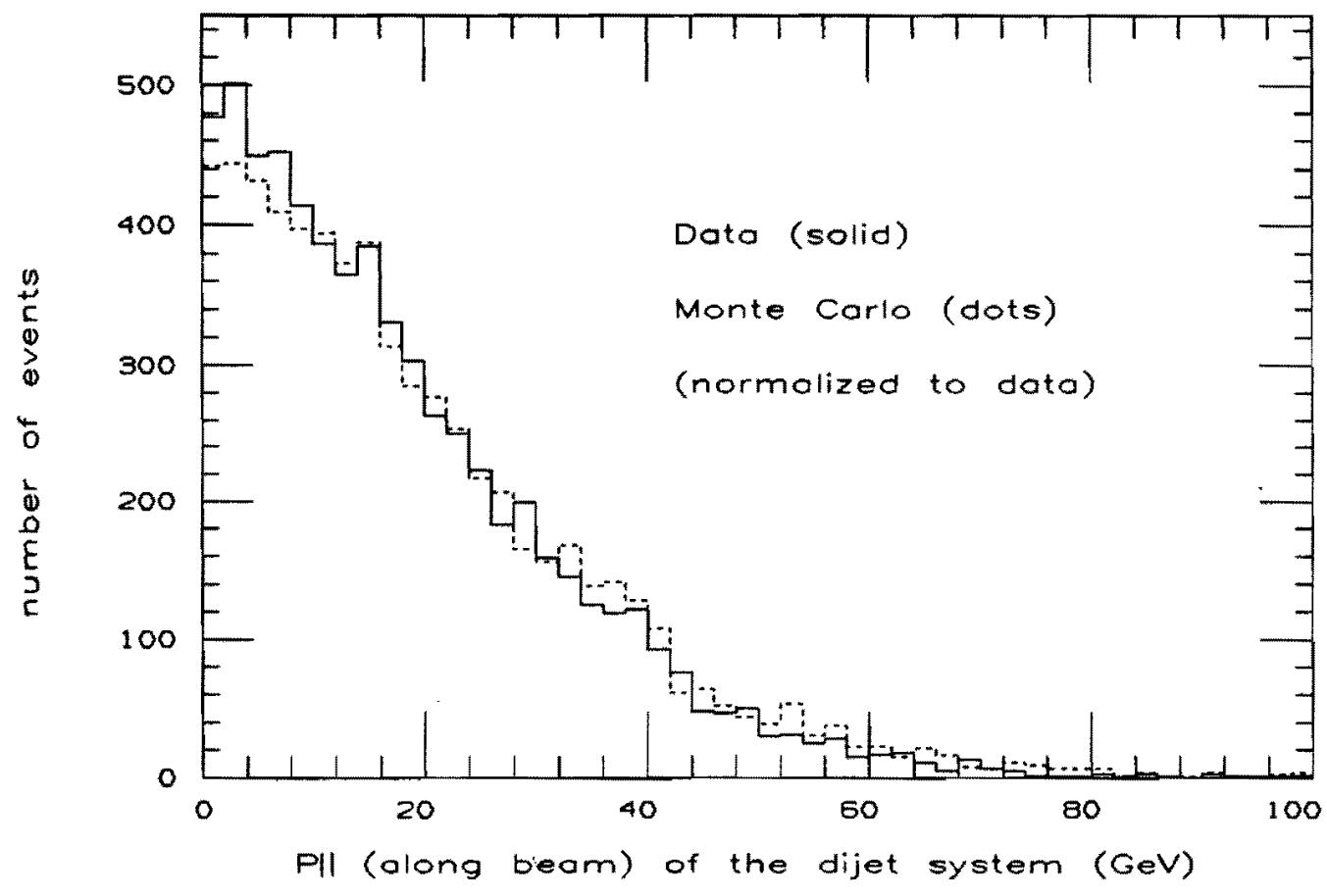

Fig. $41(b)$ Pll of the central dijet system Dota: $m=18.11, \quad \sigma=14.57 ;$ MC: $m=19.45, \quad \sigma=15.78$. 


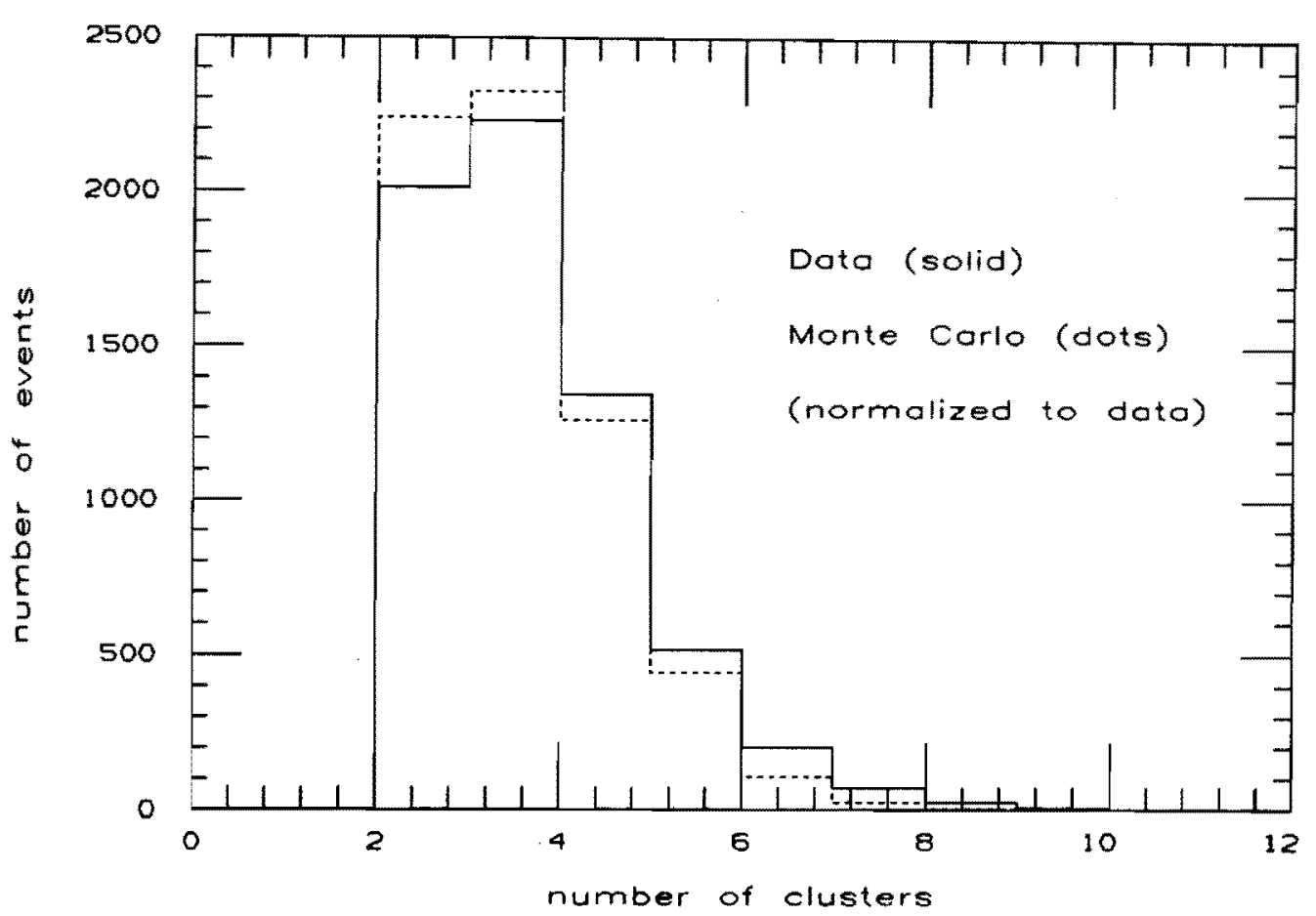

Fig. 42 Number of clusters in central dijet event

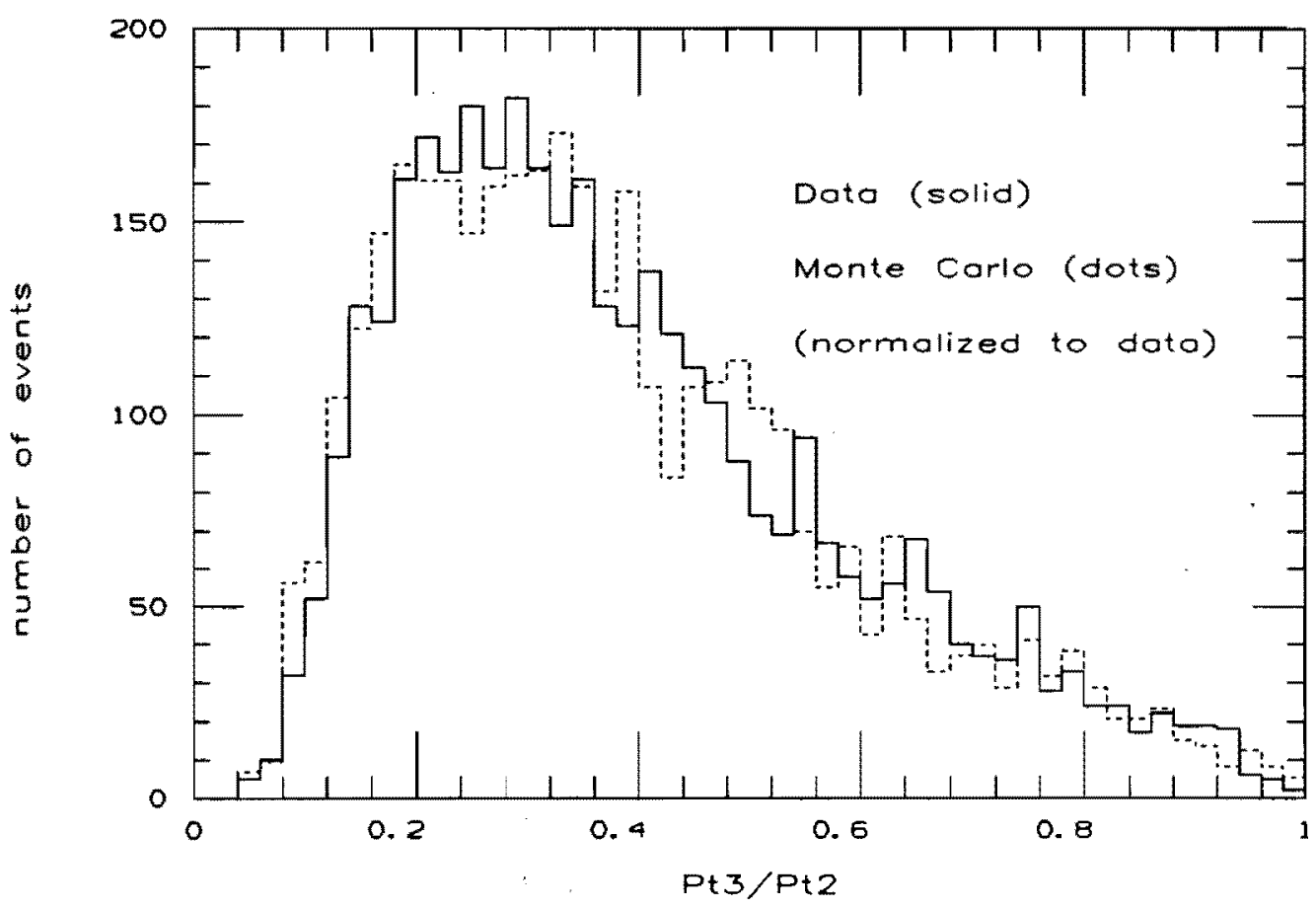

Fig. 43 Pt ratios of the 3 rd and 2 nd highest Pt jets in central dijet event. 


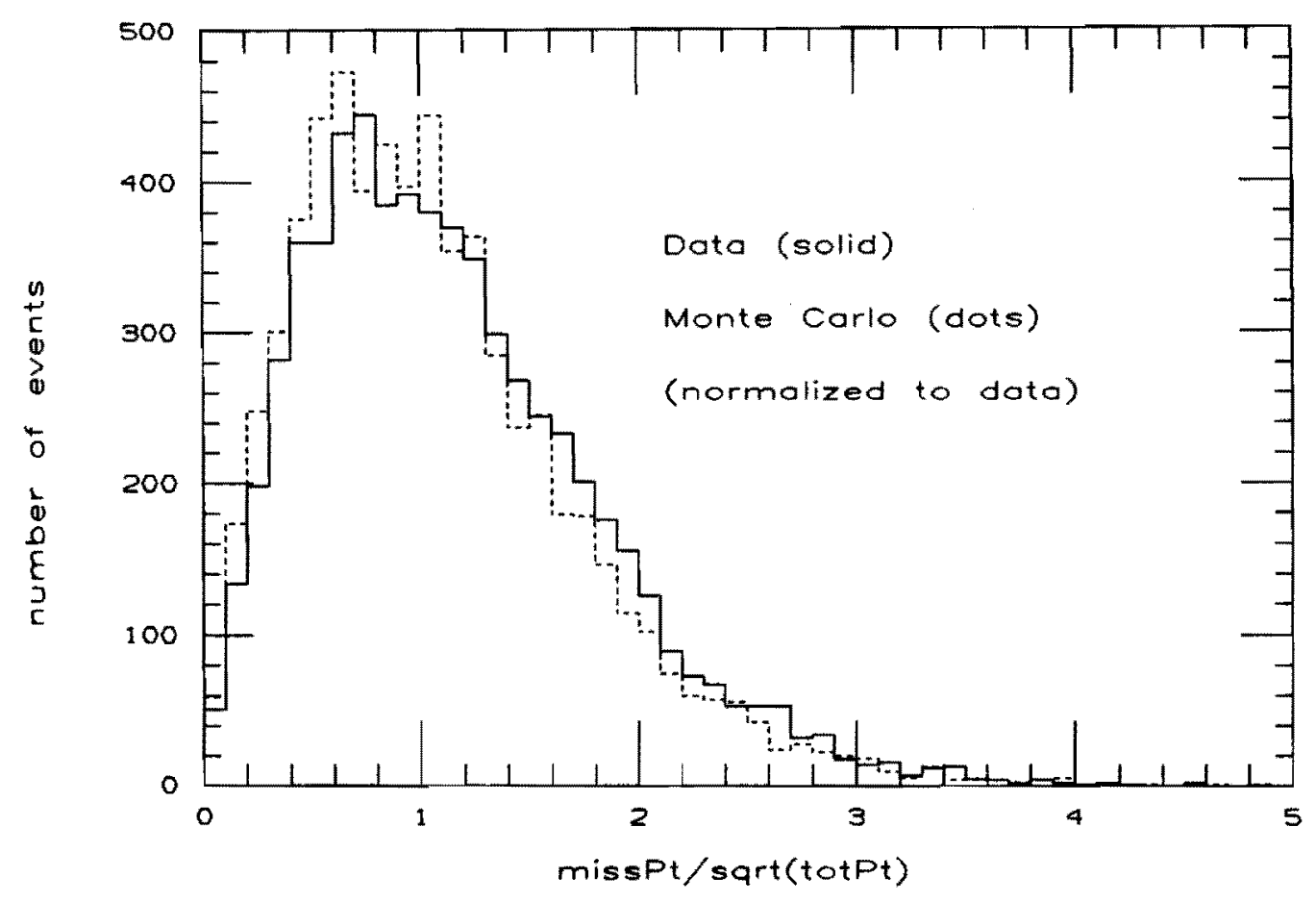

Fig. 44(a) Missing Pt in central dijet event.

Data: $m=1.15, \quad \sigma=0.67 ;$ MC: $m=1.08, \sigma=0.65$.

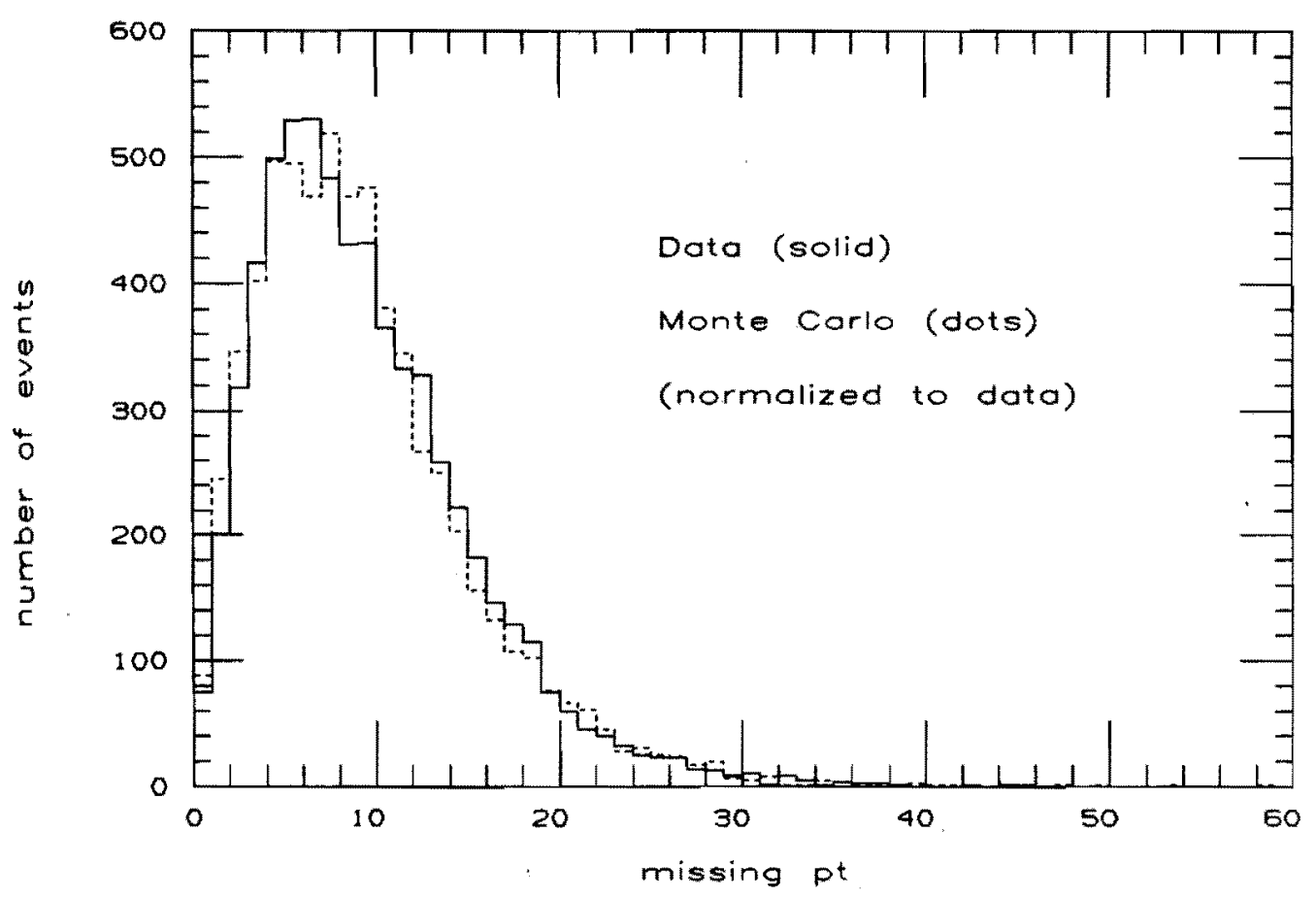

Fig. $44(b)$ Missing $P t$ in central dijet event.

Dato: $m=9.44, \sigma=5.84$; $M C: m=9.37, \sigma=6.08$. 


\section{CHAPTER 7}

\section{MDNTE CARLO STUDY OF JET ENERGY CORRECTION AND RESOLUTION}

The jet energy measured by the calorimeters only tells the first part of the story. This energy must be corrected for several detector and clustering effects: cracks in the detector, leakage due to the finite number of radiation lengths in the calorimeters, nonlinear response of the calorimeters, and the limited cone size and tower threshold in the clustering algorithm. These effects must be determined by Monte Carlo studies.

\subsection{The Sources of Jet Energy Loss}

The effects of cracks in the detector and leakage out of the back of the calorimeters are important. Cracks are between tower modules, including the steel skins of the wedges $(0.47 \mathrm{~cm})$, spaces inside the wedge for light guides and wave length shifters $(1.11 \mathrm{~cm}$ wide for CEM, $2.10 \mathrm{~cm}$ for CHA), and the big gap between the two central arches (the $90^{\circ}$ crack). These cracks constitute $13 \%$ of the total solid angle in the central detector. As a jet contains more than 10 particles on average (from an average of 10 particles for $20 \mathrm{GeV}$ jets to 30 particles for $200 \mathrm{GeV}$ jets according to ISAJET), some of them would enter cracks and lose the entirety or a large part of their energy. On the other hand, leakage occurs if showers are never developed ("punch through") or developed so late that they are not well contained inside the sampling volume.

The nonlinearity of the calorimeter response needs some explanation. Even for a perfect detector (no cracks and with infinite radiation lengths), it is inherent in any calorimeter based on particle showering and equipped with a absorber having different responses to hadronic and electromagnetic cascades. A hadron induced shower has both an EM cascade happening at the atomic level and an hadronic cascade at the nuclear level. The hadronic component has a lower particle yield (due to nuclear binding 
energy, neutrinos, particle masses and smaller cross sections etc.), giving rise to lower pulse heights (proportional to the number of minimum ionizing particles passing through the sampling regions). To illustrate further [41], if the energy fraction of the showering hadron that goes into the EM cascade increases logarithmically as $a \log (E)$ and the yield ratio of the hadronic cascade and EM cascade is $\beta$, then the pulse height (PH) would be given by $\mathrm{PH} / \mathrm{E}=\beta+(1-\beta) \alpha \log (\mathrm{E})$ with value 1.0 at $50 \mathrm{GeV}$ for the CDF calorimeters because they were calibrated with $50 \mathrm{GeV}$ electrons (CEM) and $50 \mathrm{GeV}$ charged pions (CHA). For $E<50 \mathrm{GeV}$, this function has a value less than 1. As jets consist of tens of particles with a wide range of energy (almost all below $50 \mathrm{GeV}$ ), a considerable amount of energy is 'lost' due to this effect. And the compensation for this can only be determined by studying Monte Carlo data with a detailed detector simulation.

Thus the accuracy of the energy dependent response in the detector simulation is crucial for finding the energy correction. By analyzing the 1987 minimum bias data with precise momentum measurements of charged tracks by the central tracking system, the CDF jet group has determined the Edependence of the response [45] and tuned the Monte Carlo accordingly [44] (Fig. 97). In the last chapter (Ch. 6), it was the tuned Monte Carlo which we compared with data.

Finally let us also say some words about the clustering effects. There is no algorithm that collects all the jet-related energy and at same time has good jet separation. Increasing the cluster cone size certainly improves the energy resolution but also results in the inclusion of more unrelated energy and degrades jet separation. The cluster cone size must be reasonably chosen by compromise. In our analysis we are mostly concerned with two-jet topology and the jet separation is not the prime factor. But to avoid the Plug calorimeter for the 1987 data (see $\mathrm{Ch}$. 4 and $\mathrm{Ch}$. 8), we have chosen a smaller cone size $R=0.7$. We will estimate how much energy is lost due to this limited cone size in a later section (Sect. 7.5). We will also estimate the effects of the tower energy threshold (to keep out spurious noise) and the extra energy from the underlying event (Appendix C) . 


\subsection{Choosing ISAJET as the Event Generator}

There are several event generators available on the HEP market (ISAJET, PYTHIA, EUROJET, COJETS/WIZJET, HERWIG etc.) and they differ in their models of fragmentation and underlying events. But we have chosen ISAJET not for any preference on jet fragmentation but for the single fact that ISAJIET has an unambiguous association of particles and partons. This makes the task easier to define a jet from hundreds of particles that almost fill up the space. Still there is ambiguity on how to define a jet from a multitude of partons (generated by final state parton showering, see Sect. B.3 in Appendix B). We can hope any differences in energy correction due to different fragmentation models are within the systematic errors we will quote later.

As we have explained in Sect. B.3, ISAJET has a mixed scheme to hadronize partons: a parton showering stage followed by independent fragmentation. The default ISAJET fragmentation parameters $a=0.96$ and $b=$ 3.0 (in the fragmentation function $f(z)=1-a+a(b+1)(1-z) b$, see Sect. B.1) were tuned to the UA1 result. But a direct comparison between CDF simulated events and real events showed disagreements in a number of aspects, e.g. the number of charged particles and particle Pt distributions etc [43]. For almost all the quantities compared in Ref. 43 except the invariant mass for charged particles, the parameters $a=0.88$ and $b=2.0$ originally proposed by Feynman \& Field [46] which were used in pre-UA1 ISAJET gave better agreement with our data. So we have used this set of parameters for our study.

\subsection{Energy Flow around Jet Axis}

So far we have discussed getting the calorimeter response from minimum bias data and comparing the jet fragmentation with jet (charged track) data. We also have compared several jet features in the last chapter (Ch. 6). But we have not said anything about the shapes or sizes of jets, which clearly determine the energy out of the clustering cone. Unless the shapes (or more precisely the energy contents within a clustering cone) of the 
Monte Carlo jets and real jets are similar, any Monte Carlo corrections would be meaningless. An important distribution to compare is the energy flow around the jet axis. We simulated a jet sample with both jets in the central rapidity region and required the dijet invariant mass to be greater than $70 \mathrm{GeV}$. We then plot the jet energy density distributions along the $\phi$ and $\eta$ strips and in the radial direction for jet Pt above $25 \mathrm{GeV}$ (Fig. 45 47). The strips span 0.8 in width (where most of the jet energies are concentrated) and stretch 3.0 in length around the jet center (all units are in the natural units of rapidity and radian for $\eta$ and $\phi$ ). We have taken energy with and without a tower threshold of $0.2 \mathrm{GeV}$ and found similar results. The agreement on the underlying energy is very striking. Although the ISAJET jets seem to be too narrow, the points of takeoff from background are very similar. Both issues concerning underlying energy and jet shape have been addressed by Frank Paige [13], who had the same conclusion as we have now but with UA1 data. We thus expect the energy out of the clustering cone and the extra energy from the underlying event to be similar in the Monte Carlo and real data. Integrating Fig. 46 from $R=0.7$ to $R=1.0$ gives a difference of $250 \mathrm{MeV}$, out of the minimum jet $\mathrm{Pt} 25 \mathrm{GeV}$. In fact, as we will see in Sect. 7.5, the energy out of the cone ranges from $8 \%$ for $30 \mathrm{GeV}$ jets to $3 \%$ for $200 \mathrm{GeV}$ jets according to the Monte Carlo, and any disagreement between the Monte Carlo and data would only give a small error in the jet energy. We also note that the clustering algorithm is not a true "fixed" cone algorithm as nearby cones are merged.

But it's not clear why ISAJET should be doing the right thing as the independent fragmentation is applied to the very soft initial state partons $(\langle\mathrm{Pt}\rangle=4 \mathrm{GeV}$ ) and cut Pomerons (Appendix $\mathrm{C}$ ).

\subsection{Monte Carlo Procedures}

\subsubsection{Event Generation}

ISAJET was modified so that the generated jet $\mathrm{Pt}$ distribution is flat. This ensures: a) a balance in jet statistics for all energy, and b) the peak and width of the measured jet energy distribution will not be distorted by a steeply falling spectrum. We also limited both scattered 
partons to be in the central rapidity range $|y|<1.0$, because our goal is to correct for the central jets only. We have two groups of samples with different Pt limits, one $20<\mathrm{Pt}<80 \mathrm{GeV}$ and the other $80<\mathrm{Pt}<200 \mathrm{GeV}$. The low Pt samples have approximately the same number of events as their high Pt counterparts. Each sample will be divided into 12 bins and each bin will have a distribution of measured energy. The somewhat arbitrary division of the samples at $80 \mathrm{GeV}$ was intended to equalize the statistics since for the higher Pt samples we will have larger bin sizes.

Further event selection involved a clustering of the Monte Carlo partons. In ISAJET the 4 partons in the initial and final states are evolved into a group of partons by parton showering (see Sect. B.3 and Appendix C). Our parton clustering only involves partons generated from the final state. The initial state radiation, which is soft and isotropic in $\phi$ and responsible for scale violating structure functions, joins the spectator quarks (beam jets) to form the underlying background. The QCD hard scattering process with scale violating structure function is considered to be decoupled from these backgrounds.

We merged the Monte Carlo partons into jets by a cone algorithm in the following way. The highest Pt partion is selected as the seed and its $\eta$ and $\phi$ are calculated from the particles which originate from it. We have to use the sum of the final particle energies as the true jet energy because the ISAJET independent fragmentation doesn't strictly conserve energy and momentum. Then a cone is drawn around the seed parton on the $\eta-\phi$ space and all partons inside this cone are included in the jet. To decide a proper cone size we have scanned a few events and concluded that a cone size 1.0 is sufficient in most cases to include all partons that topologically belong to a jet while retaining good jet separation. After the inclusion of more partons, the jet axis is recalculated and a new cone is drawn to search for more. This is repeated until no new member can be found and a jet is settled. And then the whole procedure is reiterated on the remaining free (not assigned to any jet) partons, starting from the highest Pt one, until every final state parton has been assigned to a jet. When parton clustering was finished, we required the two highest $\mathrm{Pt}$ jets to be in the 
pseudo-rapidity range $|\eta|<0.7$, which is the kinematic region used in our data analysis.

To study the corrections for the $90^{\circ}$ crack region, a special procedure was adopted to generate jets pointing right at the crack and having the same vertex distribution as observed in real data (Fig. 63 in Ch. 8). In this special procedure the event vertices were determined during generation according to the observed gaussian distribution. The pseudorapidity $\eta_{0}$ that would make a jet point to the crack center was then calculated from the vertex position and the radius of the calorimeter by $\eta_{0}=-\log \left(\tan \left(\theta_{0} / 2.\right)\right)$, where $\theta_{0}=\cos ^{-1}\left(-z / \sqrt{z^{2}+215^{2}}\right)(r=215 \mathrm{~cm}$ was chosen as it is on the face of the central hadron calorimeter). Jets were then generated around this $\eta_{0}$ by a modified ISAJET. We then defined jets by running the parton clustering described above and required that the two highest $P t$ jets had to be near the crack center within 0.1 unit of $\eta$.

Each sample contains about 5000 events. We must emphasize that the above event selection was not motivated by any physics reason but only for economy's sake as each event takes about 30 VAX8600 CPU seconds to simulate the calorimeters alone.

\subsubsection{Detector Simulation}

The generated events were then passed to a detailed detector simulation. The event vertices for the non-crack samples were randomly selected from a gaussian distribution $(\sigma=35 \mathrm{~cm}$, mean $=3.7 \mathrm{~cm})$ which is similar to that observed in data. The vertices in the crack samples have been determined in the generation phase (rather than during detector simulation) according to the same Gaussian distribution. Every particle was stepped through the detector region and various effects (dE/dx, decay, conversion, scattering and showering etc.) were applied according to known probabilities. The CDF shower simulation is discussed in Appendix $E$. The final results are tower $A D C$ counts exactly as for real data.

\subsubsection{Corrections and Resolutions}

The Monte Carlo data are put through the standard CDF jet analysis (JETCLU algorithm, Sect. 6.1.3). The jet energy corrections and resolutions 
were obtained by comparing the true jet energy with the calorimeter cluster energy, where the 'true' jet energy is defined by the parton clustering described above (Sect. 7.4.1). We used only the two highest Pt jets because the others are mostly due to wide angle initial or final state bremsstrahlung. The matching of jets and clusters is done by requiring the $\Delta R$ (in $\eta-\phi$ space) between them to be less than 0.4 . All usable jets must not have any particles lost due to simulation error (because the simulation is so complicated that error does occur occasionally, and when this happens the rest of the event is skipped by the program) and the correspondence between jets and clusters must be one-to-one. The jets also can not have any other nearby jets within $R=1.5$, so that a cluster can get energy from only one jet. We then make distributions (Fig. 49) of the differences between the measured $E(P)$ and the true $E(P)$ for jets in various $E(P)$ ranges, which are $5 \mathrm{GeV}$ wide for $20<E(P)<80 \mathrm{GeV}$ and $10 \mathrm{GeV}$ for $80<$ $E(P)<200 \mathrm{GeV}$. The mean (or peak) of each distribution tells the mean measured $E(P)$, and the width of the distribution gives the jet $E(P)$ resolution. At low jet energy the distributions look fairly gaussian because the cluster energy is limited from below (no negative energy). But as the jet energy increases, leaving more room for jets to undergo more severe energy loss, long tails of badly measured jets appear at the low end. These tails have two origins: a) some high energy particles have lost a large fraction of energy to cracks or have not showered, and b) the parton clustering disagrees with calorimeter clustering. But any misassignment of partons should not be included for the energy correction because a calorimeter cluster comes from a group of partons and we ask how to correct the energy for these partons. With this consideration, and judging that we should be correcting for the majority of jets, which are jets clustered inside the (approximate) gaussian form, we feel it is legitimate to cut off these long tails. Fortunately we can further comfort ourselves by observing that these tails are at low energy end and because the jet Pt spectrum is falling like a rock, they can be regarded as 'lost' (detection inefficiency) and yet add insignificantly to the lower Pt region. 


\section{a) $E(P)$ Corrections}

The resulting mean cluster energies versus various jet energies are plotted in Fig. 50. The abscissa of these plots is the mean of the true jet energies in a range of 5 or $10 \mathrm{GeV}$, and the ordinate is the mean of the measured energies. The nonlinearity of the response is visible on these plots. To find smooth functions that can be used for data analysis we have tried various fits: vertical parabola $\left(y=a x^{2}+b x+c\right)$, horizontal parabola $\left(x=a y^{2}+b y+c\right)$ and 2 -straight-line. A vertical parabola fit may be better than the horizontal one because the bending in the plot is more prominent in the low energy region. The response looks fairly linear for jet energies from $100 \mathrm{GeV}$ up to $200 \mathrm{GeV}$, making all parabolic fits suspicious for energies over $200 \mathrm{GeV}$. The 2-straight-line fit is the simplest and has the smallest $\chi^{2}$ among the fits. Another possibility is to have a straight line fit for high Pt and a spline fit at low Pt, matching their values and slopes so that there is smooth transition from one region to the other. But the 2-straight-line fits seem adequate. We $I$ ist the $E$ and $P$ corrections for the crack and non-crack regions in Table 8 .

\section{b) $E(P)$ Resolutions}

The resolutions for various jet energies and momenta are plotted in Figs. $51^{\circ}$ - 52. Recall that they were derived from the sigmas of truncated distributions (with long tails cut off). To get corrected resolutions, the sigmas ( $\sigma$ 's) have been multiplied by $\partial E_{r e a l} / \partial E_{\text {measured }}$ (For example if the energy is corrected linearly by $a \mathrm{E}_{\text {meaured }}+\beta$, then the corrected resolution is $a \cdot \sigma_{\text {measured }}$ ) Although we have generated many events for high statistics, with more than 400 jets in each distribution, the bin-to-bin fluctuations are larger than the statistical error bars would indicate. Again we have tried various fits: a) $a \sqrt{E}+\beta, b) a \mathrm{E}^{\beta}$, where $0.5<\rho<1$, and c) $a \sqrt{E}+\beta E$. Fit a) has the smallest $\chi^{2}$, but its non-positive values at low energy pose potential problems in the procedure of resolutionunsmearing the measured spectra (see Sect. 8.4.1). So we have chosen to use fit b).

c) Dijet Mass Resolution 
The dijet mass $\left(M_{J}\right)$ resolutions are determined by a similar technique. The $E$ and $P$ corrections found previously are used back on the same sample and the dijet masses are calculated from the corrected energy and momenta $\left(=\operatorname{sqrt}\left[\left(E_{1}+E_{2}\right)^{2}-\left(P_{1}+P_{1}\right)^{2}\right]\right)$. As in the case of $E$ and $P$, we use only the two highest $\mathrm{Pt}$ jets and both jets must have $|\eta|<0.7$. The measured masses are compared with the true masses, and a separate distribution of the mass differences is made for each $10 \mathrm{GeV}$ mass range in the interval $40<\mathrm{MJJ}_{\mathrm{J}}<$ $160 \mathrm{GeV}$ and each $20 \mathrm{GeV}$ range in the interval $160<\mathrm{MJJ}_{\mathrm{JJ}}<400 \mathrm{GeV}$. The resulting mass resolutions are plotted in Fig. 54. Among the 3 types of fits mentioned in last section, the best (the smallest $\chi^{2}$ ) was found to be $a E^{\beta}$. The $E, P$, and $M_{J J}$ resolutions are listed in Table 9 .

\section{d) Angular Resolution}

We can also study the jet angular resolutions by making the distributions of $\Delta r$ (in $\eta-\phi$ space) between the true jet and measured cluster axes. The results are plotted in Fig. 55, where we see the angular resolution gets better for higher Pt jets.

\subsection{How Energy Is Lost}

To get some idea about how the jet energy is lost, we generated samples with both the minimum bias background (from spectator quarks) and initial state radiation turned off. An event was selected if it contained exactly two jets (defined by the parton clustering described in Sect. 7.4.1) and both jets are in the rapidity region $|\eta|<0.7$. The pure calorimeter response, including the effects of cracks, longitudinal leakage and nonlinearity, is then determined by simply assigning the towers to the closest jet. This results in approximately half of the calorimeter being assigned to a jet. Three other effects can also be studied with this sample: the energy out of the cone, loss due to tower threshold, and the extra energy from the underlying event. We will label the pure calorimeter response as the response $\# 1$, and two more responses made by running the cluster finding (rather than just apportioning the energy by distance) with tower energy thresholds 0.0 and $0.2 \mathrm{GeV}$ as responses $\# 2$ and \#3. We also 
have the total response from the last section where the MC sample contains underlying energy; this is called response \#4. To summarize, the responses are (see Fig. 56):

\#1: tower threshold $=0.0 \mathrm{GeV}$, approximately half of the calorimeter assigned to the jet, and no simulation of the underlying event;

\#2: tower threshold $=0.0 \mathrm{GeV}$, clustering cone size $=0.7$, and no simulation of the underlying event;

\#3: tower threshold $=0.2 \mathrm{GeV}$, clustering cone size $=0.7$, and no simulation of the underlying event;

\#4: tower threshold $=0.2 \mathrm{GeV}$, clustering cone size $=0.7$, and with simulation of the underlying event.

The calorimeter energy that lies too far away from the jet center is just the difference between the calorimeter energy and the energy reported by the clustering algorithm with tower threshold $0.0 \mathrm{GeV}, i, e . \# 1-\$ 2$. The energy loss due to the tower threshold is given by the difference between the two clusterings which differ only in their tower thresholds, i.e. 2 \#3. And the difference between the measured jet energies from a sample with underlying energy and a sample without underlying energy, i.e. $\$ 4-\$ 3$, gives the extra energy from the underlying event.

So we have made 4 response curves by fitting vertical parabolas to the 4 response data (Fig. 56). From these 4 curves the various jet energy losses (and fractional losses) are derived and plotted in Fig. 57. Not surprisingly the calorimeter response is by far the dominant factor because it is the combined effects of major losses in our experiment: nonlinearity, cracks and leakage. The observation that energy out of the clustering cone rises with energy, but fractional loss decreases, is also not surprising. The loss due to tower threshold is a minor effect, about half a GeV for all energies. What is somehow surprising is the rise of extra energy from the underlying event. Studies on jet events [47] seem to indicate that the background energy remains constant irrespective of jet energy. We do not know exactly the origin of the rise in ISAJET, but will note one thing: we have treated initial state radiations, which are isotropic in $\phi$, as part of the underlying background, and there might be some correlation between these soft radiations and their emitters -- the initial state partons that 
make hard collisions to produce high $P t$ jets. But the rise caused by this correlation should be compensated to some extent by the fact that after a high Pt scattering the remaining system (spectator quarks) has lower energy. In ISAJET the fragmentation parameters for cut Pomerons are made energy dependent by hand, $b=b_{0}+b_{1} \ln (s)$, in order to account for the observed increase in $\mathrm{dn} / \mathrm{dy}$ in minimum bias events. Although a lower number of particles sharing the Pomeron energy might result in higher calorimeter response due to nonlinearity, it might not have so much to do with the rise of the underlying energy. We need to study higher Pt events in order to see if the rise is wrong.

\subsection{Uncertainty in the Jet Energy Correction}

Because the spectra we are measuring are very steep indeed, any shift in the horizontal direction (energy scale) will have a big effect on the vertical scale (cross sections). Yet the energy scale is a very difficult issue. The difficulty is clear as we are dealing with multiparticle systems (jets) which lose up to nearly a quarter of their energy due to the effects of cracks, leakage and nonlinearity. The study of nonlinearity is a big subject by itself, the tuning of the detector simulation is another. Still these are not the only sources of unçertainty. The jet fragmentation affects the energy scale because the more particles which share the jet energy the lower the calorimeter response would be due to nonlinearity. The neutral to charged particle ratio (N/C) also has its effect through the difference in the calorimeter response to $e, \gamma$ and charged $\pi$ 's. Thus it is important to know how far the real energy scale can go from the "optimum" values we determined previously. We have special samples to study these:

a) We generated events with the default ISAJET fragmentation parameters $a=0.96, b=3.0$ and found energy corrections according to this fragmentation. The systematic error due to fragmentation is then taken as the difference between this set of correction and that according to the tuned ISAJET (with $a=0.88$ and $b=2.0$ ).

b) A tuned ISAJET sample (the one without underlying energy) was divided into two parts, one with jets having $N / C$ ratios in the upper $50 \%$ and the 
other in the lower $50 \%$. We then estimated the error in the jet energy due to $N / C$ ratio from the departure in detector response for either part of the sample from the optimum values.

c) We simulated the tuned ISAJET sample with the calorimeter responses at their lower limits as set by the study of minimum bias events, and determined the uncertainty due to calorimeter response.

Five response curves are plotted in Fig. 58, from which various uncertainties contributing to the total systematic error in the energy scale are derived and plotted in Fig. 59. Fragmentation can change the energy scale by $7 \%$ at $30 \mathrm{GeV}$, down to $3 \%$ at $200 \mathrm{GeV}$. The calorimeter response gives a $5 \%$ error at $30 \mathrm{GeV}$, down to $1 \%$ at $200 \mathrm{GeV}$. The uncertainty due to the charged to neutral ratio turns out to be a minor one. The uncertainty in the CDF simulation (face response including the crack and non-crack regions) is $3.6 \%$ according to Kuhlmann [43].

\subsection{Uncertainty in the Jet Energy Resolution}

We have seen that even by sending many jets of known energy into the calorimeter (more than 400 entries in each of the distributions in Fig. 49), we still have large bin-to-bin fluctuations in the determination of the detector resolution (Fig. 51 - 55). In fact, a change in the fragmentation parameters from the default ISAJET to the tuned ISAJET increases the resolution by about $5 \%$, which is somehow surprising. This fact diminishes the hope that one could place a target before a pion beam to produce a group of particles which mimic jets and determine the energy scale and resolution by measurement. The composition of jets also plays a role. The increase in resolution due to fragmentation is not hard to understand (once we have discovered $i t$ ) because as the number of particles becomes lower the loss of one particle results in the loss of a bigger fraction of energy.

\subsubsection{The UA2 Technique of Determining Pt Resolution from Data}

Then how to compare our Monte Carlo resolution with data? There is a technique invented by UA2 [48] to determine the energy resolution from data 
by exploiting the fact that $\mathrm{Pt}$ is approximately balanced in two jet events. The balance is only approximate because the two partons that make the hard collision can get a transverse kick due to initial state radiation. So the apparent imbalance in the Pt of the measured jets has two origins, one due to physics processes and one from finite detector resolution. To isolate the contribution from pure resolution, UA2 suggested that one decompose the measured $\mathrm{Kt}$ of the two jet system into 2 orthogonal components, one (called $K_{\eta}$ in the $\eta$-direction) parallel to the bisector of the transverse directions of the two jets and one perpendicular (i.e. along the 'thrust' axis of the two jets, and called $K_{\xi}$ along the $\xi$-direction). They are given by (Fig. 60)

$$
\begin{aligned}
& K_{\eta}=\left(P t_{1}+P t_{2}\right) \cos (\Delta \phi / 2) \\
& K_{\xi}=\left(P t_{1}-P t_{2}\right) \sin (\Delta \phi / 2),
\end{aligned}
$$

where $\Delta \phi$ is the azimuthal angle between the leading 2 jets and is close to $\pi$. The $K_{\xi}$ component is sensitive to both detector resolution and the physical transverse kick of the two parton system, while $K_{\eta}$ is primarily due to the physical kick because the resolution effect is suppressed by a factor $\sin ((x-\phi) / 2)$. The angular error $\delta$ in the jet direction is typically $-3^{\circ}$ and its contribution to $K_{\eta}\left(-\sqrt{2} \cdot P_{t} \cdot \delta\right)$ is expected to be small compared to $K_{\eta}-2 \cdot P t \cdot \cos (\Delta \phi / 2)$. So the distribution of $K_{\eta}$ can be used to measure the magnitude of the physical kick, which should have equal $\eta$ - and $\xi$ - components on average if the kicks are isotropic in $\phi$. Thus although the $\xi$-component of kick can not be separated from $K_{\xi}$, it can be statistically subtracted and the $\mathrm{Pt}$ resolution can be determined from the expression

$$
\sigma_{P t}=\sqrt{\left(\left\langle K_{\xi}^{2}\right\rangle-\left\langle K_{\eta}^{2}\right\rangle\right) / 2}
$$

(Note: the subtraction of $\sigma^{\prime} s$ in quadrature doesn't require the distributions to be gaussian. In our case the distributions are only required to be peaked at 0 .)

This technique has been pursued in CDF, first vigorously by Rick St. Denis [49] and then others. But it is important to show that this method really works. The questions are: how to select two jet events and whether it $f$ inds the right resolution. We set out to understand these better by studying Monte Carlo data. Even if our detector simulation does not 
reproduce the real resolution, the method itself should give a resolution which is characteristic of the Monte Carlo sample and can be determined by our previous method, i.e. from the distribution of the measured jet energy.

The dijet selection is not a trivial issue because different selections affect $K_{\xi}$ and $K_{\eta}$ significantly and result in somehow different answers. Two cuts can be applied to select dijet events, one is on the azimuthal angle $\Delta \phi$ between leading 2 jets and the other on the Pt of the third jet (Pt3). A cut on $\mathrm{Pt} 3$ restricts the strength of the radiation and thus restricts the transverse kicks. Because we do not require the distribution of kicks to be gaussian, any reasonable cut on Pt3 can be used in principle. On the other hand, we need be more careful about cutting on $\Delta \phi$ because if the $\Delta \phi$ cut is too close to $\pi$ we will be cutting off high $\eta$ kicks (kicks in the $\eta$ direction) but not high $\xi$ kicks. In other words, the contribution to $K_{\eta}$ and $K_{\xi}$ would not be the same if we have the same large $\Delta \phi$ (close to $\pi$ ) cut for both $K_{\eta}$ and $K_{\xi}$. If $\Delta \phi$ is too small we may include multijets or dijets with hard final state bremsstrahlung; furthermore $K_{\xi}$ is no longer a good measurement of $P t$ resolution because $K_{\xi}=(P t 1-P t 2) \sin (\Delta \phi / 2)$ would differ from (Pt1 - Pt2) significantly.

The choice is not easy and we have tried two kinds of $\phi$ cuts: a) $140^{\circ}$ or $150^{\circ}$ for both $K_{\eta}$ and $K_{\xi}$, and b) $120^{\circ}$ for $K_{\eta}$ and $160^{\circ}$ for $K_{\xi}$. We also have two different Pt3 cuts: fixed $10 \mathrm{GeV}$ or $5 \mathrm{GeV}$ for all Pt. The potential problem with the fixed $P_{3}$ cut is that the angular error may dominate the physical kick for higher Pt events. But a variable Pt cut is not without problems either because the physical kicks may not grow with Pt and we are more likely to pick up events with multijets or hard final state bremsstrahlung. The differences in resolution due to different choices are compared in Figs. 61 - 62 and they are found to be considerably larger in real data than in Monte Carlo. This might be due to the various noise problems in the gas systems which are not simulated. We can not conclude from the Monte Carlo study which choice is better because the differences are relatively small. But we have found that the Monte Carlo resolution is most consistent with the resolution determined from real data by the UA2 technique using a Pt3 cut of $10 \mathrm{GeV}$ and same $\Delta \phi$ cut of $150^{\circ}$ for both $K_{\eta}$ and $K_{\xi}$ (denoted as $[10,150,150]$ in Fig. 62(a)). In fact for two back-to-back 
jets with identical $\mathrm{Pt}=20 \mathrm{GeV}$ a $10 \mathrm{GeV}$ kick would produce $\Delta \phi>152^{\circ}$. The cuts $[5,150,150]$ appear to be even more plausible since a tighter cut on Pt3 should give cleaner dijet events, but the resulting resolution is slightly lower than the Monte Carlo resolution.

In summary, we judge from the above study that the UA2 technique seems to work well on Monte Carlo data where all calorimeters function perfectly; but for our 1987 data the technique has a large uncertainty due to dijet selection. It gives a resolution $0-20 \%$ (depending on dijet selection) lower than the Monte Carlo resolution.

\subsubsection{What Error Will Be Quoted for Resolution?}

Then what would we say about the uncertainty in energy resolution? The default ISAJET gives resolution about $5 \%$ lower than the tuned ISAJET. The untruncated Monte Carlo distributions (Fig. 49) would give a resolution higher for high energy jets. The UA2 technique can give a resolution for real data up to $20 \%$ lower than what we determined from Monte Carlo. For higher Pt jets there is yet no comparison and the resolutions can be quite different. In view of these uncertainties, we conclude that the uncertainty in the resolution should be taken as high as $20 \%$ (of the resolution). 
Table. 5 Real and measured jet energy in Monte Carlo. $\operatorname{Err}=\sigma / \sqrt{\mathrm{N}}$, where $\sigma$ is the width of the distribution and $N$ the number of entries.

\begin{tabular}{|c|c|c|c|c|c|c|c|c|}
\hline \multicolumn{3}{|c|}{$\left|\eta_{d}\right|<0.05$} & \multicolumn{3}{|c|}{$0.05<\left|\eta_{d}\right|<0.10$} & \multicolumn{2}{|c|}{$0.1<\left|\eta_{d}\right|<1.0$} & \multirow[b]{2}{*}{ err } \\
\hline & measured & err & real & measured & err & real & measured & \\
\hline 22.722 & 14.309 & 0.259 & 22.674 & 14.408 & 0.303 & 22.729 & 15.322 & 0.187 \\
\hline 27.610 & 17.462 & 0.252 & 27.528 & 18.320 & 0.267 & 27.560 & 19.433 & 0.206 \\
\hline 32.484 & 20.414 & 0.315 & 32.541 & 21.668 & 0.334 & 32.523 & 23.070 & 0.224 \\
\hline 37.408 & 23.903 & 0.386 & 37.650 & 26.224 & 0.330 & 37.492 & 26.908 & 0.256 \\
\hline 42.545 & 28.370 & 0.404 & 42.503 & 29.622 & 0.400 & 42.438 & 31.197 & 0.285 \\
\hline 47.358 & 31.586 & 0.397 & 47.298 & 33.535 & 0.440 & 47.334 & 35.213 & 0.303 \\
\hline 52.606 & 35.212 & 0.458 & 52.551 & 37.475 & 0.469 & 52.399 & 38.458 & 0.333 \\
\hline 57.684 & 38.684 & 0.512 & 57.502 & 41.521 & 0.483 & 57.588 & 42.799 & 0.379 \\
\hline 62.573 & 42.951 & 0.551 & 62.599 & 46.151 & 0.540 & 62.538 & 47.563 & 0.387 \\
\hline 67.429 & 45.179 & 0.582 & 67.558 & 49.209 & 0.516 & 67.466 & 51.540 & 0.423 \\
\hline 72.433 & 47.764 & 0.714 & 72.480 & 54.032 & 0.593 & 72.527 & 55.551 & 0.438 \\
\hline 77.393 & 53.195 & 0.821 & 77.436 & 57.809 & 0.761 & 77.368 & 58.615 & 0.514 \\
\hline 84.365 & 59.576 & 0.645 & 84.731 & 64.307 & 0.600 & 84.588 & 64.793 & 0.414 \\
\hline 94.781 & 67.060 & 0.918 & 94.961 & 70.057 & 0.790 & 94.678 & 74.390 & 0.554 \\
\hline 105.293 & 74.591 & 0.939 & 105.069 & 81.001 & 0.714 & 105.013 & 83.199 & 0.630 \\
\hline 115.161 & 83.633 & 1.061 & 115.353 & 90.733 & 0.963 & 114.926 & 91.684 & 0.622 \\
\hline 125.143 & 88.635 & 1.197 & 125.225 & 96.359 & 1.034 & 124.972 & 101.346 & 0.689 \\
\hline 134.858 & 98.153 & 1.315 & 134.912 & 106.766 & 1.038 & 134.946 & 110.157 & 0.731 \\
\hline 145.052 & 105.681 & 1.527 & 144.948 & 114.033 & 1.171 & 144.985 & 119.664 & 0.782 \\
\hline 154.904 & 112.975 & 1.574 & 155.186 & 121.658 & 1.120 & 155.036 & 129.704 & 0.927 \\
\hline 164.735 & 121.259 & 1.738 & 165.146 & 130.628 & 1.299 & 165.122 & 137.899 & 0.832 \\
\hline 175.176 & 128.213 & 1.881 & 175.001 & 140.492 & 1.334 & 174.769 & 146.855 & 1.000 \\
\hline 184.781 & 133.037 & 1.857 & 184.409 & 147.298 & 1.611 & 184.771 & 155.216 & 0.867 \\
\hline 194.850 & 140.210 & 2.282 & 194.511 & 157.256 & 1.939 & 195.040 & 166.469 & 1.003 \\
\hline
\end{tabular}


Table 6 Real and measured jet momenta in Monte Carlo. Err $=\sigma / \sqrt{N}$, where $\sigma$ is the width of the distribution and $N$ the number of entries.

\begin{tabular}{|c|c|c|c|c|c|c|c|c|}
\hline \multicolumn{3}{|c|}{$\left|\eta_{d}\right|<0.05$} & \multicolumn{3}{|c|}{$0.05<\left|\eta_{d}\right|<0.10$} & \multicolumn{2}{|c|}{$0.1<\left|\eta_{d}\right|<1.0$} & \multirow[b]{2}{*}{ err } \\
\hline real & measured & err & real & measured & err & real & measured & \\
\hline 22.570 & 15.019 & 0.233 & 22.610 & 15.695 & 0.258 & 22.577 & 16.317 & 0.163 \\
\hline 27.497 & 18.381 & 0.252 & 27.492 & 19.402 & 0.270 & 27.576 & 20.526 & 0.190 \\
\hline 32.349 & 21.037 & 0.310 & $32 \cdot 568$ & 23.238 & 0.314 & 32.522 & 24.391 & 0.207 \\
\hline 37.446 & 26.006 & 0.369 & 37.527 & 27.181 & 0.315 & 37.379 & 28.361 & 0.230 \\
\hline 42.544 & 29.654 & 0.360 & 42.598 & 30.948 & 0.398 & 42.586 & 32.810 & 0.265 \\
\hline 47.462 & 33.436 & 0.409 & 47.682 & 35.883 & 0.381 & 47.523 & 36.801 & 0.276 \\
\hline 52.487 & 36.813 & 0.466 & 52.480 & 39.395 & 0.402 & 52.420 & 40.095 & 0.324 \\
\hline 57.550 & 39.968 & 0.506 & 57.590 & 43.757 & 0.493 & 57.499 & 45.201 & 0.325 \\
\hline 62.548 & 43.966 & 0.533 & 62.632 & 46.874 & 0.458 & 62.613 & 50.032 & 0.359 \\
\hline 67.495 & 47.052 & 0.621 & 67.489 & 52.743 & 0.497 & 67.427 & 52.898 & 0.376 \\
\hline 72.465 & 51.258 & 0.759 & 72.342 & 55.381 & 0.683 & 72.561 & 56.992 & 0.436 \\
\hline 77.348 & 56.439 & 0.787 & 77.436 & 59.741 & 0.724 & 77.320 & 61.875 & 0.455 \\
\hline 84.582 & 61.257 & 0.813 & 84.783 & 66.442 & 0.597 & 84.746 & 68.169 & 0.422 \\
\hline 94.619 & 70.760 & 0.794 & 94.736 & 73.914 & 0.731 & 94.838 & 76.195 & 0.523 \\
\hline 105.026 & 76.488 & 0.947 & 104.899 & 83.143 & 0.749 & 105.171 & 86.371 & 0.515 \\
\hline 114.921 & 85.150 & 1.033 & 114.896 & 92.227 & 0.870 & 114.931 & 95.511 & 0.615 \\
\hline 124.779 & 91.030 & 1.176 & 124.831 & 99.147 & 0.926 & 125.076 & 104.621 & 0.599 \\
\hline 134.806 & 102.717 & 1.189 & 135.080 & 110.271 & 0.986 & 135.236 & 113.755 & 0.685 \\
\hline 145.123 & 109.400 & 1.488 & 144.747 & 115.031 & 1.159 & 144.961 & 123.396 & 0.676 \\
\hline 154.971 & 116.438 & 1.459 & 154.906 & 125.634 & 1.036 & 155.078 & 133.608 & 0.781 \\
\hline 165.170 & 124.041 & 1.794 & 164.490 & 135.419 & 1.116 & 164.593 & 140.375 & 0.820 \\
\hline 175.091 & 130.053 & 1.764 & 174.928 & 145.090 & 1.288 & 174.959 & 149.785 & 0.853 \\
\hline 184.946 & 135.034 & 2.318 & 185.149 & 151.316 & 1.791 & 184.851 & 159.714 & 0.901 \\
\hline 194.429 & 147.080 & 2.508 & 194.026 & 162.634 & 1.941 & 194.981 & 171.432 & 0.988 \\
\hline
\end{tabular}


Table 7 Energy, momentum and dijet mass resolutions in Monte Carlo. $\sigma_{E}=c \sigma$ and err $=c \sigma / \sqrt{2 N}$, where $c=d E($ corrected) $/ \mathrm{dE}$ (measured), $\sigma$ is the width of the distribution and $N$ is the number of entries. Similar definitions for $P$ and $M$ resolutions.

\begin{tabular}{|c|c|c|c|c|c|c|c|c|}
\hline \multicolumn{2}{|c|}{$0.1<\left|\eta_{d}\right|<1.0$} & \multirow[b]{2}{*}{ err } & \multicolumn{2}{|c|}{$0.1<\left|\eta_{d}\right|<1.0$} & \multirow[b]{2}{*}{ err } & \multicolumn{2}{|c|}{$\left|\eta_{1}\right|,\left|\eta_{2}\right|<0.7$} & \multirow[b]{2}{*}{ err } \\
\hline$E$ & $\sigma_{E}$ & & $P$ & $\sigma_{\mathrm{p}}$ & & $M_{J J}$ & $\sigma_{M}$ & \\
\hline 22.729 & 4.807 & 0.165 & 22.577 & 4.407 & 0.140 & 45.272 & 6.994 & 0.269 \\
\hline 27.560 & 5.791 & 0.183 & 27.576 & 5.314 & 0.164 & 54.942 & 8.227 & 0.302 \\
\hline 32.523 & 6.444 & 0.198 & 32.522 & 5.994 & 0.177 & 65.208 & 10.050 & 0.350 \\
\hline 37.492 & 7.414 & 0.226 & 37.379 & 6.575 & 0.198 & 74.950 & 10.442 & 0.366 \\
\hline 42.438 & 8.184 & 0.252 & 42.586 & 7.816 & 0.227 & 84.931 & 11.931 & 0.421 \\
\hline 47.334 & 8.920 & 0.268 & 47.523 & 7.747 & 0.237 & 95.101 & 12.690 & 0.451 \\
\hline 52.399 & 9.363 & 0.294 & 52.420 & 8.803 & 0.278 & 104.840 & 13.829 & 0.525 \\
\hline 57.588 & 10.634 & 0.335 & 57.499 & 9.311 & 0.279 & 115.129 & 15.099 & 0.570 \\
\hline 62.538 & 10.776 & 0.341 & 62.613 & 9.786 & 0.309 & 124.897 & 14.818 & 0.536 \\
\hline 67.466 & 10.625 & 0.329 & 67.427 & 9.402 & 0.288 & 134.566 & 18.020 & 0.688 \\
\hline 72.527 & 10.464 & 0.341 & 72.561 & 10.063 & 0.334 & 144.739 & 19.481 & 0.754 \\
\hline 77.368 & 11.711 & 0.399 & 77.320 & 10.325 & 0.349 & 154.959 & 19.530 & 0.791 \\
\hline 84.588 & 12.702 & 0.322 & 84.746 & 11.675 & 0.324 & 169.405 & 19.688 & 0.643 \\
\hline 94.678 & 14.194 & 0.431 & 94.838 & 13.216 & 0.400 & 189.717 & 23.082 & 0.813 \\
\hline 105.013 & 15.782 & 0.489 & 105.171 & 13.391 & 0.394 & 210.207 & 25.591 & 0.912 \\
\hline 114.926 & 16.348 & 0.484 & 114.931 & 14.722 & 0,471 & 230.085 & 27.102 & 0.990 \\
\hline 124.972 & 16.104 & 0.535 & 125.076 & 13.960 & 0.458 & 249.752 & 25.394 & 0.893 \\
\hline 134.946 & 17.244 & 0.568 & 135.236 & 16.424 & 0.524 & 269.343 & 27.494 & 1.082 \\
\hline 144.985 & 19.249 & 0.608 & 144.961 & 16.247 & 0.518 & 289.693 & 30.071 & 1.160 \\
\hline 155.036 & 21.797 & 0.720 & 155.078 & 17.723 & 0.598 & 310.141 & 33.268 & 1.340 \\
\hline 165.122 & 19.532 & 0.647 & 164.593 & 18.547 & 0.628 & 329.979 & 34.680 & 1.382 \\
\hline 174.769 & 21.467 & 0.777 & 174.959 & 18.457 & 0.653 & 349.798 & 32.670 & 1.361 \\
\hline 184.771 & 19.537 & 0.674 & 184.851 & 19.238 & 0.690 & 369.889 & 33.637 & 1.489 \\
\hline 195.040 & 20.390 & 0.779 & 194.981 & 18.044 & 0.757 & 389.434 & 37.043 & 1.774 \\
\hline
\end{tabular}


Table 8 Formulae for the $E$ and $P$ corrections. $E_{m}$ and $P_{m}$ are the measured, and $E$ and $P$ the corrected energy and momentum.

\begin{tabular}{|c|c|c|}
\hline detector $\eta$ & fit function & $\chi^{2} /$ Ndeg \\
\hline$\left|\eta_{d}\right|<0.05$ & $\begin{array}{l}E_{m}=2.86 \cdot 10^{-4} E^{2}+0.693 E-1.95 \\
P_{m}=2.11 \cdot 10^{-4} P^{2}+0.725 P-1.78\end{array}$ & $\begin{array}{l}31.94 / 24 \\
38.55 / 24\end{array}$ \\
\hline $0.05<\left|\eta_{d}\right|<0.1$ & $\begin{array}{l}E=1.271 E_{m}+4.524 \text { if } E_{m}<57.11 \\
E=1.188 E_{m}+9.264 \text { if } E_{m}>57.11 \\
E_{m}=3.30 \cdot 10^{-4} E^{2}+0.759 E-3.03 \\
P=1.241 P_{m}+3.499 \text { if } P_{m}<60.91 \\
P=1.159 P_{m}+8.494 \text { if } P_{m}>60.91 \\
P_{m}=3.62 \cdot 10^{-4} P^{2}+0.773 P-2.15\end{array}$ & $\begin{array}{l}5.93 / 12 \\
15.81 / 12 \\
20.70 / 24 \\
13.52 / 12 \\
13.87 / 12 \\
23.16 / 24\end{array}$ \\
\hline $0.1<\left|\eta_{d}\right|<1.0$ & $\begin{array}{l}E=1.250 E_{m}+3.562 \text { if } E_{m}<65.45 \\
E=1.099 E_{m}+13.451 \text { if } E_{m}>65.45 \\
E_{m}=6.06 \cdot 10^{-4} E^{2}+0.745 E-1.78 \\
P=1.215 P_{m}+2.783 \text { if } P_{m}<66.234 \\
P=1.083 P_{m}+11.506 \text { if } P_{m}>66.234 \\
P_{m}=5.53 \cdot 10^{-4} P^{2}+0.775 P-1.39\end{array}$ & $\begin{array}{l}10.88 / 12 \\
5.25 / 12 \\
21.66 / 24 \\
14.48 / 12 \\
13.77 / 12 \\
29.50 / 24\end{array}$ \\
\hline
\end{tabular}

Table 9 Energy, momentum and dijet mass resolutions.

\begin{tabular}{ll}
\hline fit function & $\chi^{2}$ for 24 d.o.f. \\
\hline$\sigma_{E}=1.838 E^{1 / 2}-3.93$ & 58.63 \\
$\sigma_{E}=0.622 E^{0.68}$ & 69.76 \\
$\sigma_{E}=0.85 E^{1 / 2}+0.056 E$ & 83.60 \\
$\sigma_{P}=1.632 P^{1 / 2}-3.31$ & 38.17 \\
$\sigma_{P}=0.587 \mathrm{P}^{0.67}$ & 46.74 \\
$\sigma_{P}=0.78 \mathrm{P}^{1 / 2}+0.05 P$ & 58.44 \\
$\sigma_{M}=2.229 \mathrm{M}^{1 / 2}-8.40$ & 42.72 \\
$\sigma_{M}=0.386 \mathrm{M}^{0.77}$ & 40.54 \\
$\sigma_{M}=0.68 \mathrm{M}^{1 / 2}+0.065 \mathrm{M}$ & 49.90
\end{tabular}




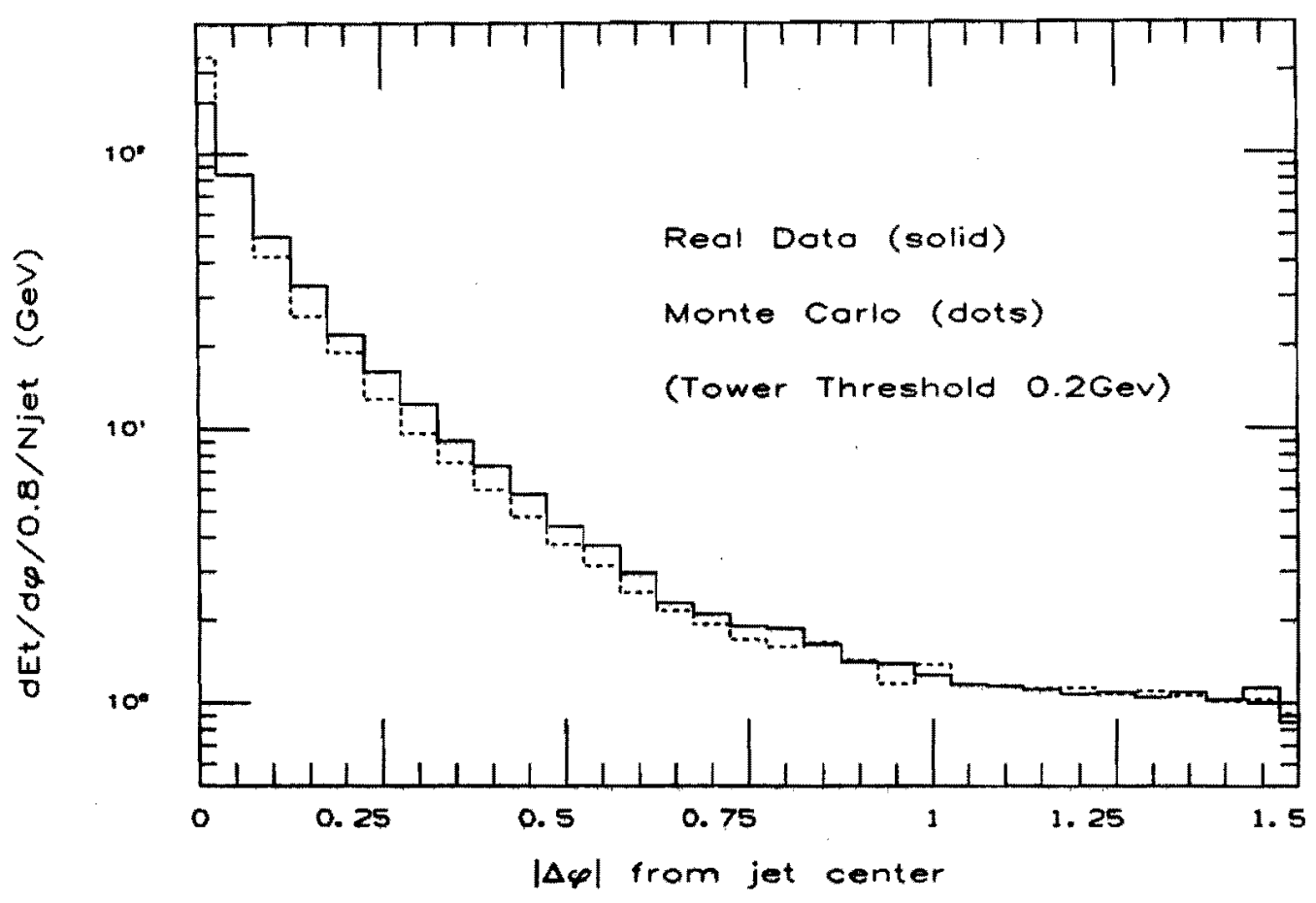

Fig. $45(a)$ Jet Et density along $\varphi$ strip of 0.8 wide in $\eta$. All jets hove Pt $\geq 25$ GeV.

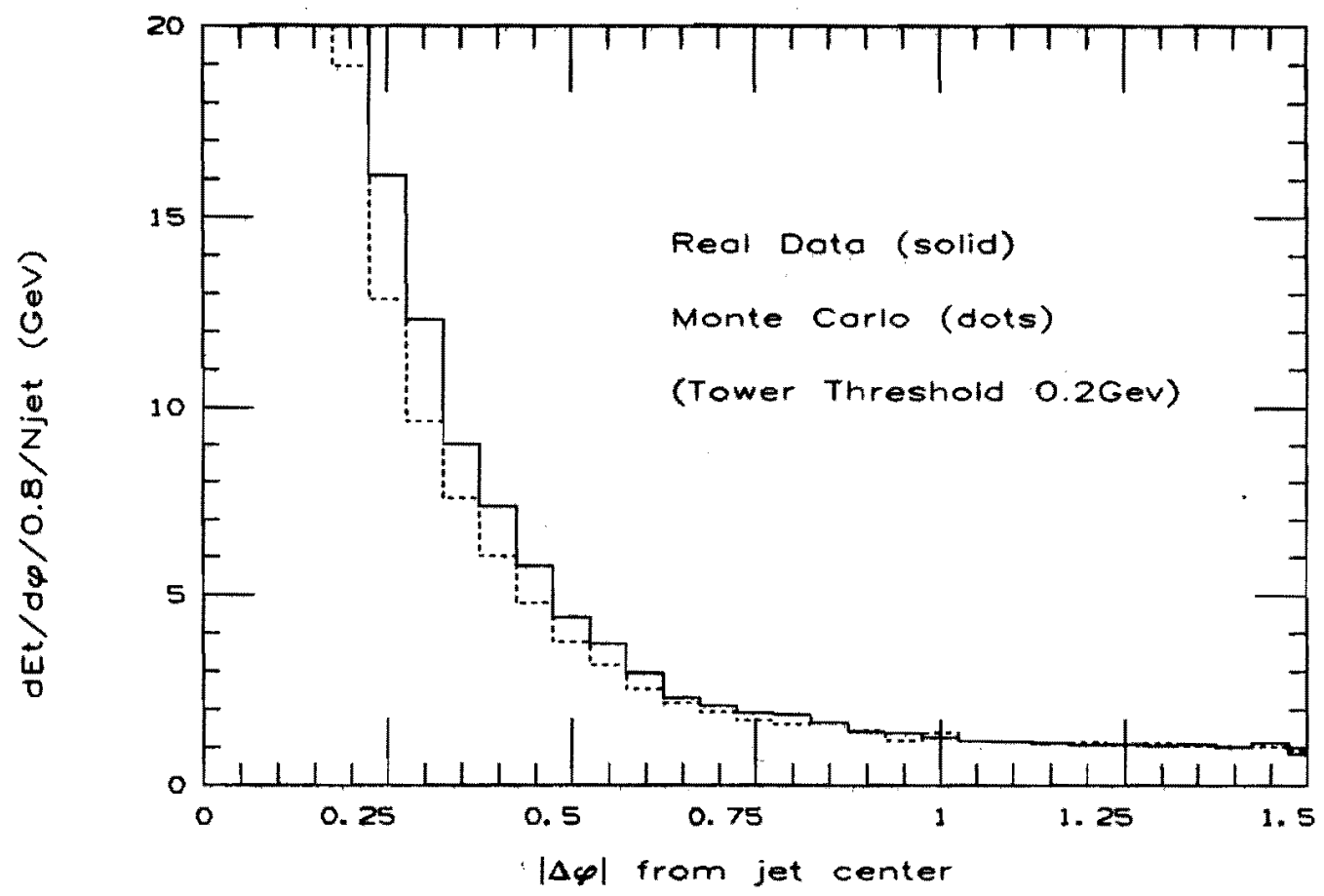

Fig. 45(b) Same as (a) but in linear scale. 


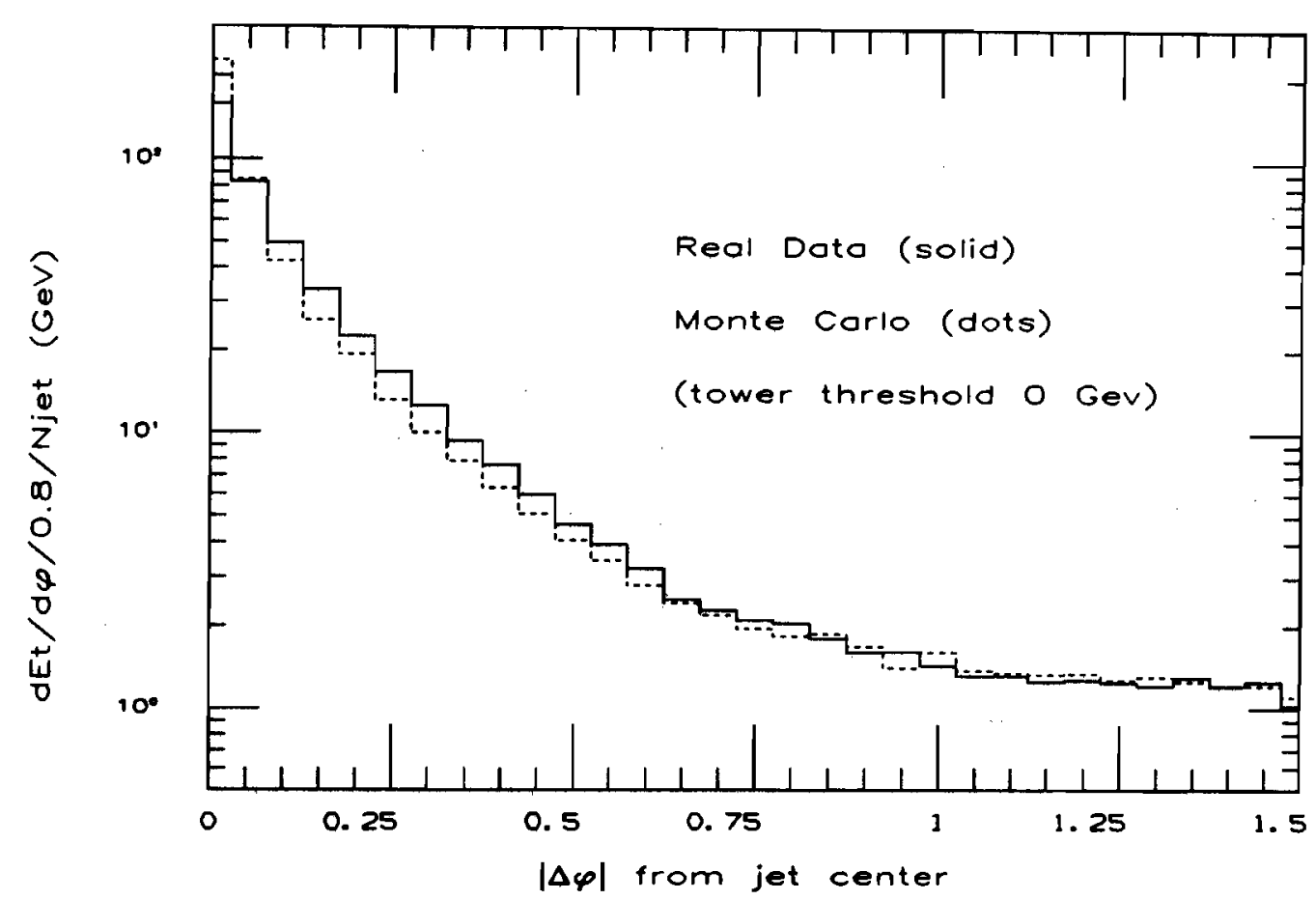

Fig. 45(c) Same as (a) but without tower threshold.

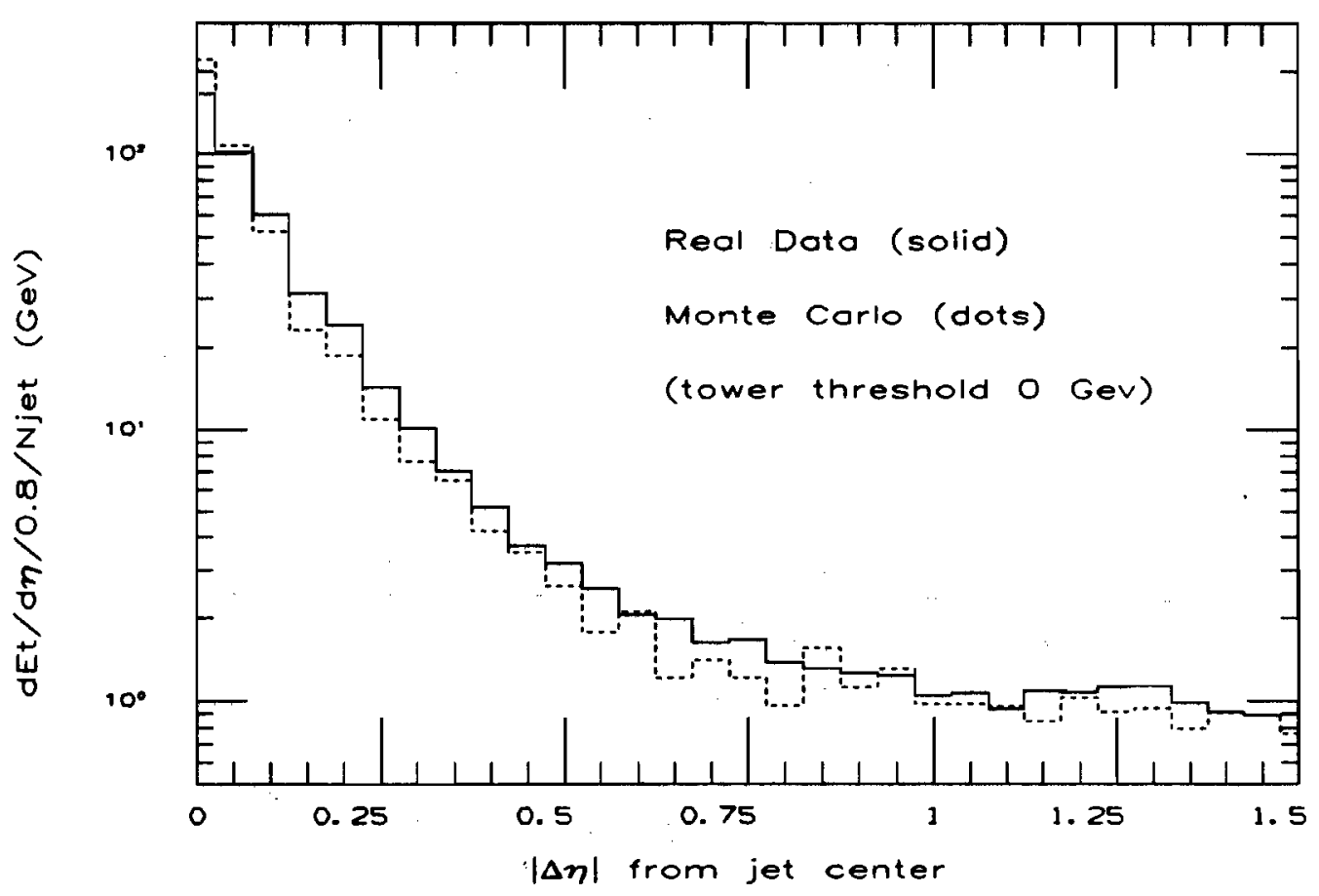

Fig. $45(d)$ Jet Et density olong $\eta$ strip of 0.8 wide in $\varphi$. All jets have Pt $\geq 25 \mathrm{GeV}$. 


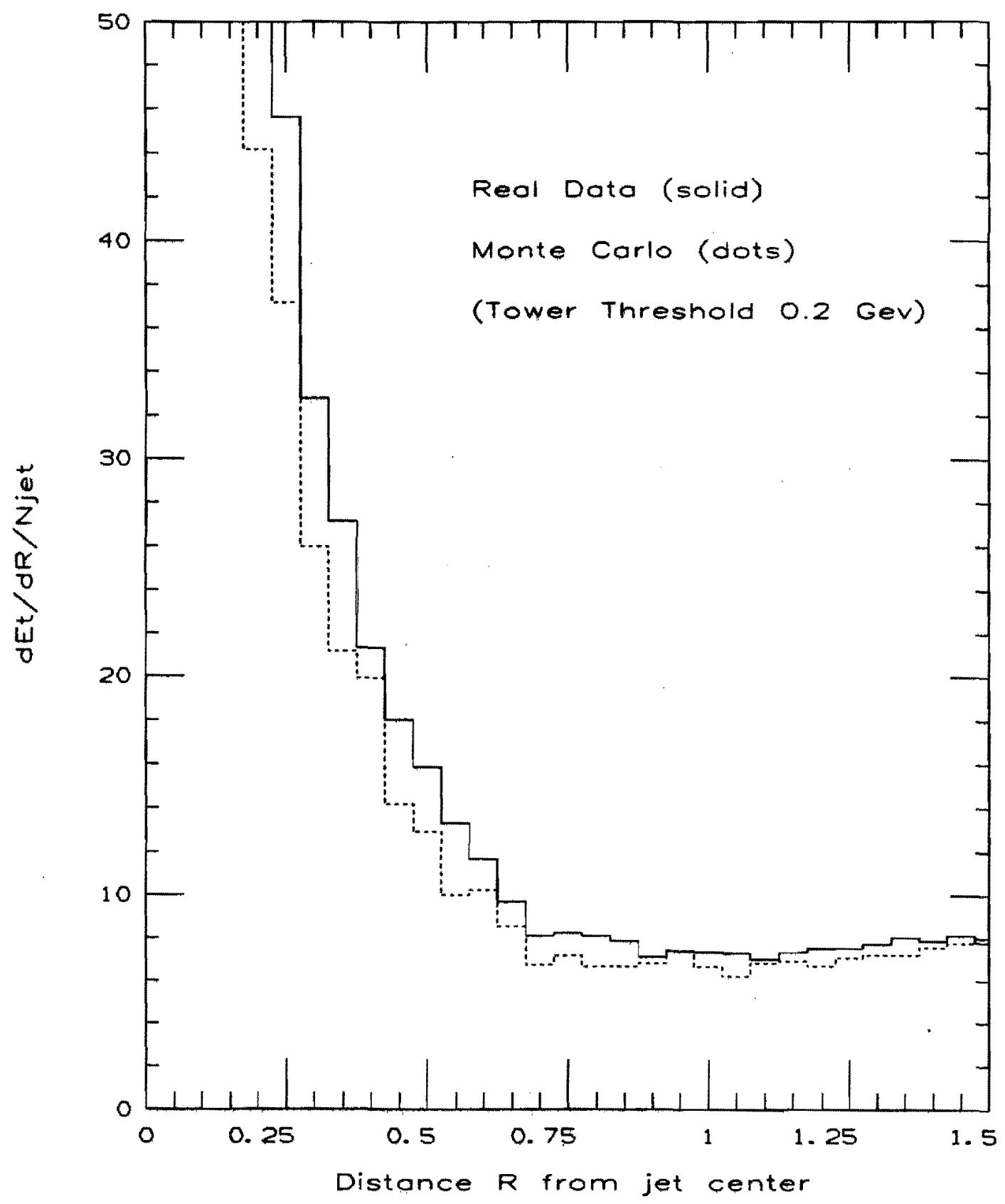

Fig. 46 Et content in rings around jet center. 


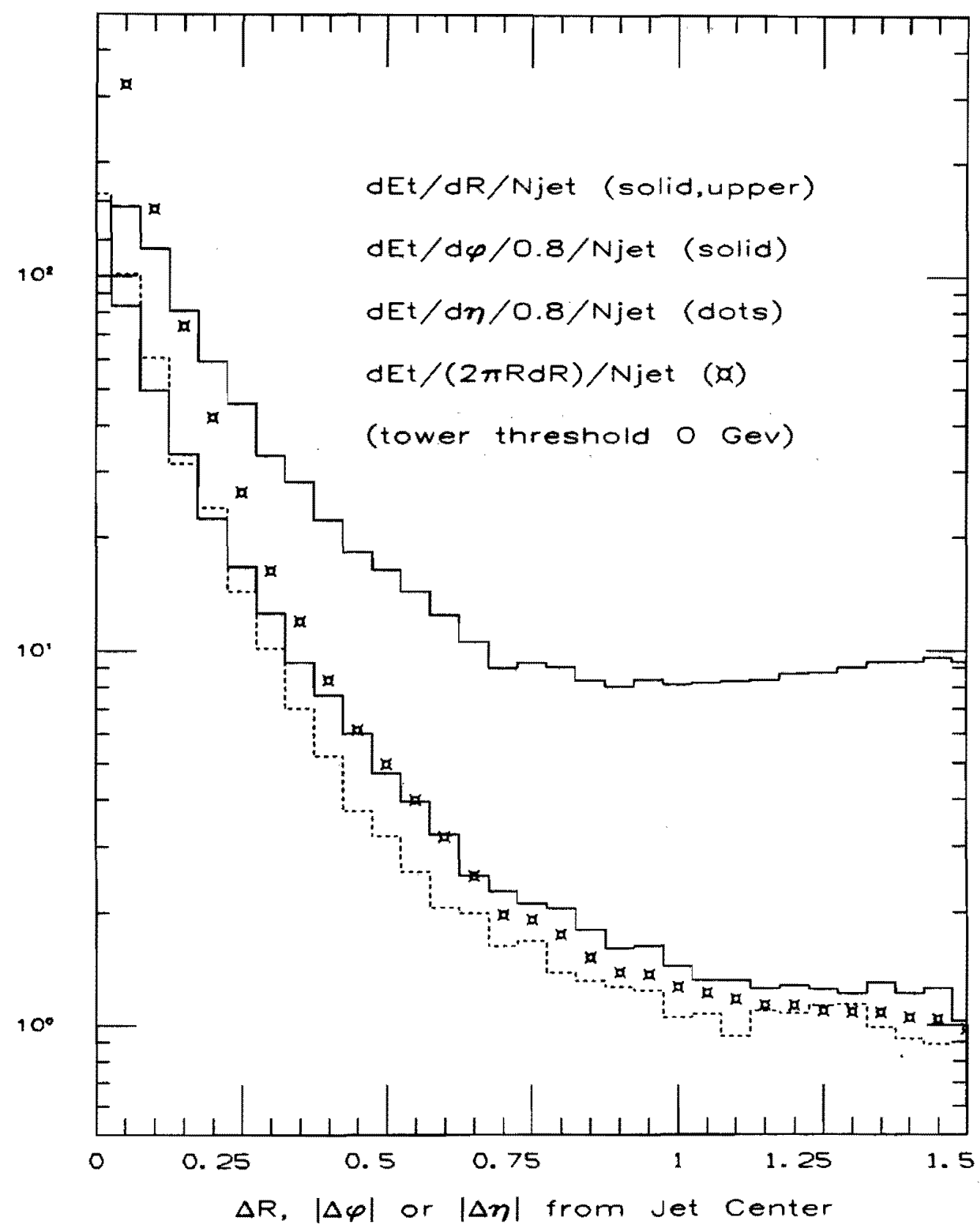

Fig. 47 Distributions of Et around jet center (data). 
118

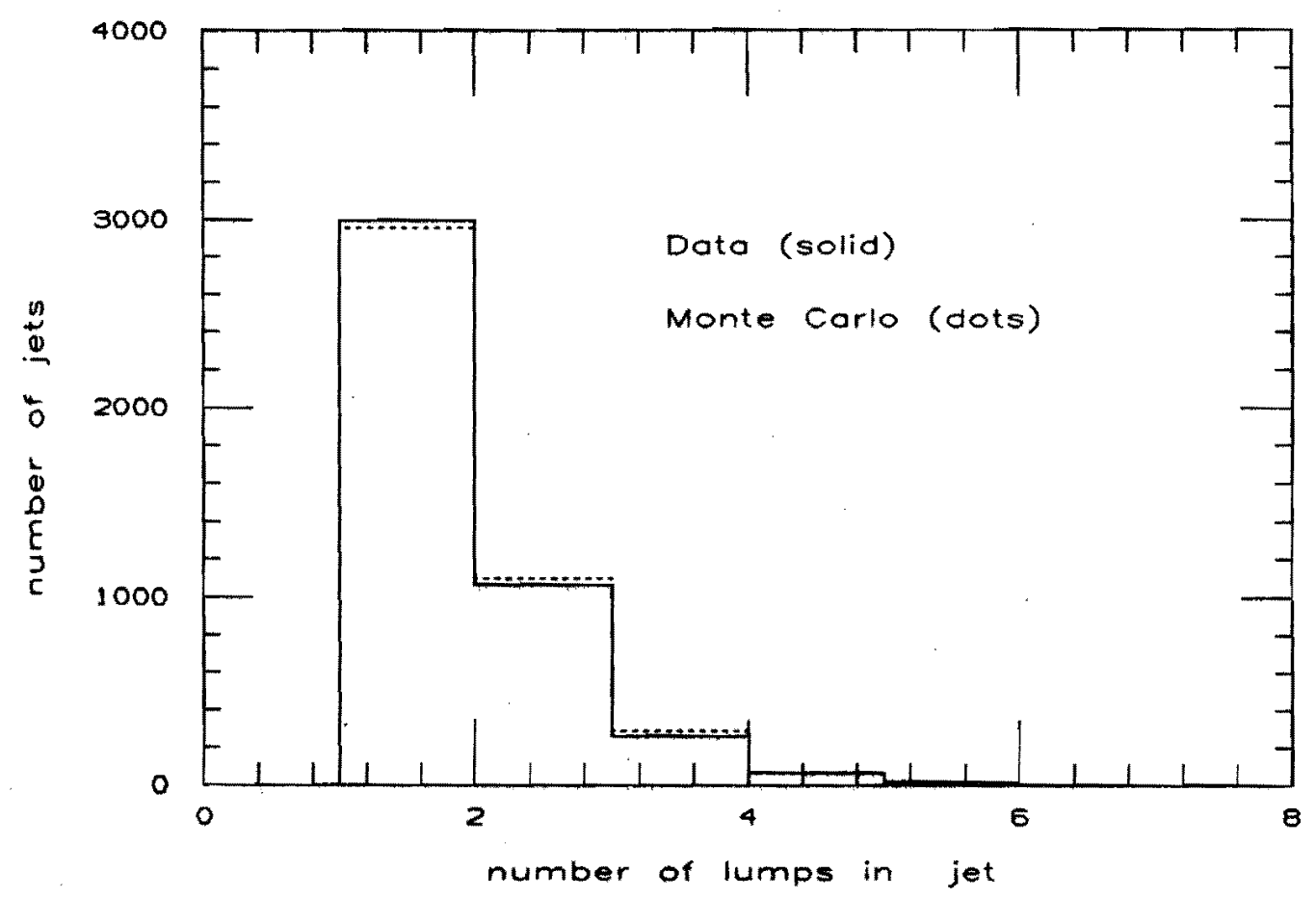

Fig. $48(a)$ The number of energy lumps in a jet ( $P t \geqq 30 G e V$ ). 


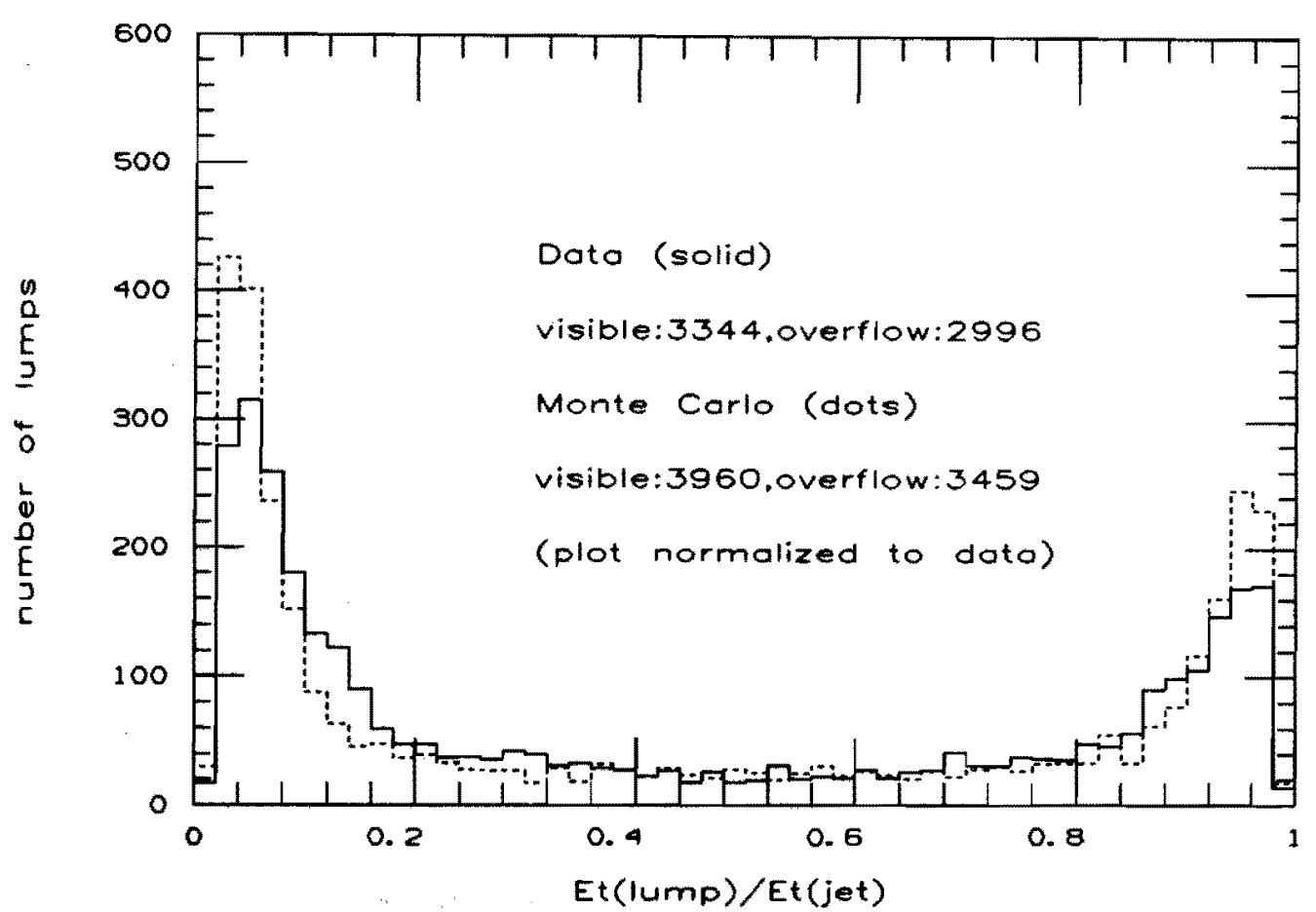

Fig. $48(b)$ The Et fractions of energy lumps in a jet ( $P t \geqq 30 G e V$ )

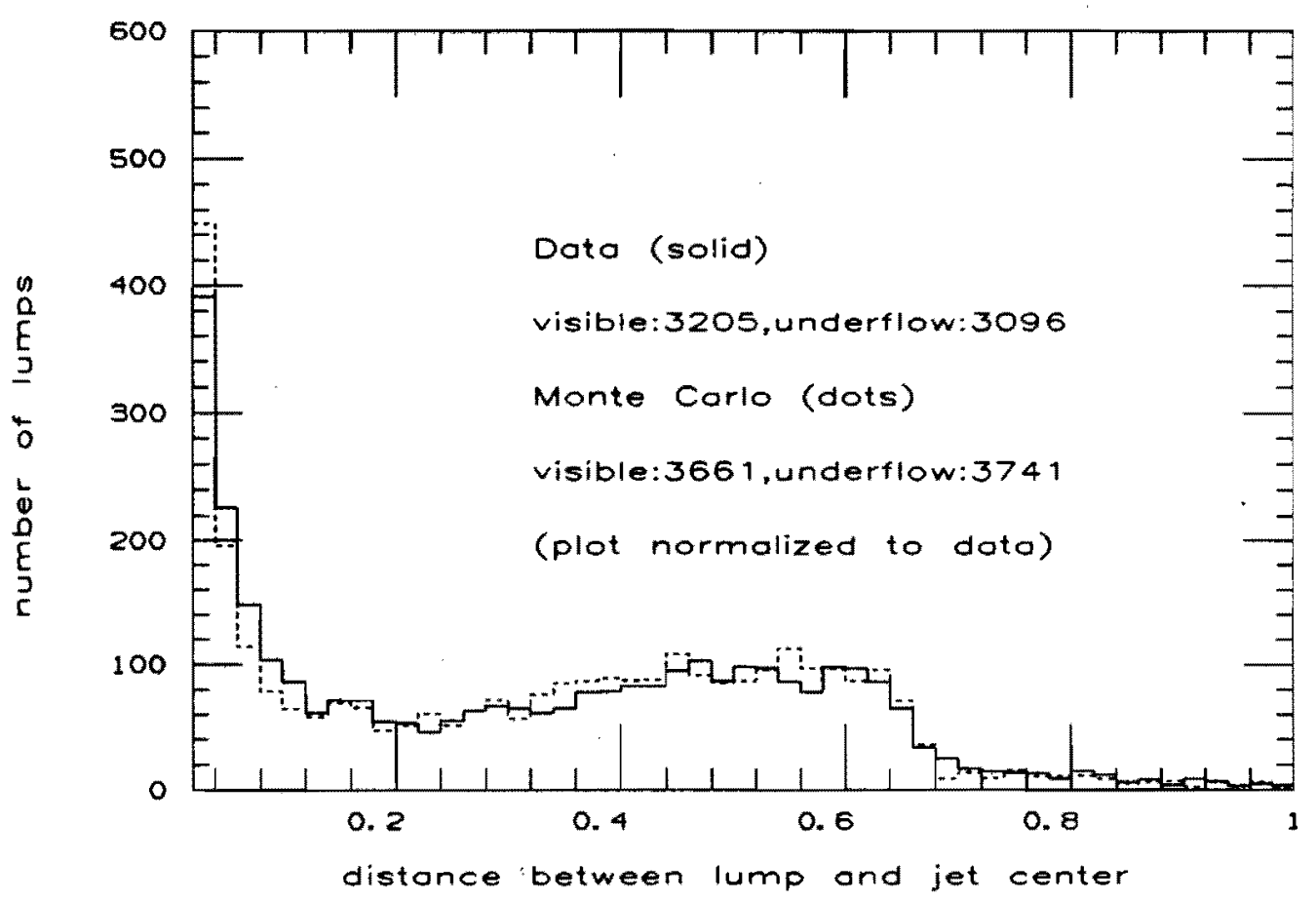

Fig. 48(C) Distances between lumps and jet (Pt $\geq 30 \mathrm{GeV}$ ) center 
Fig. 49 The distributions of the differences between the measured and real jet energies for jets in various energy ranges. For example the distribution \#70 has real jet energies starting from $70 \mathrm{GeV}$, and up to $75 \mathrm{GeV}$ which is the starting real jet energy for distribution \#75. 

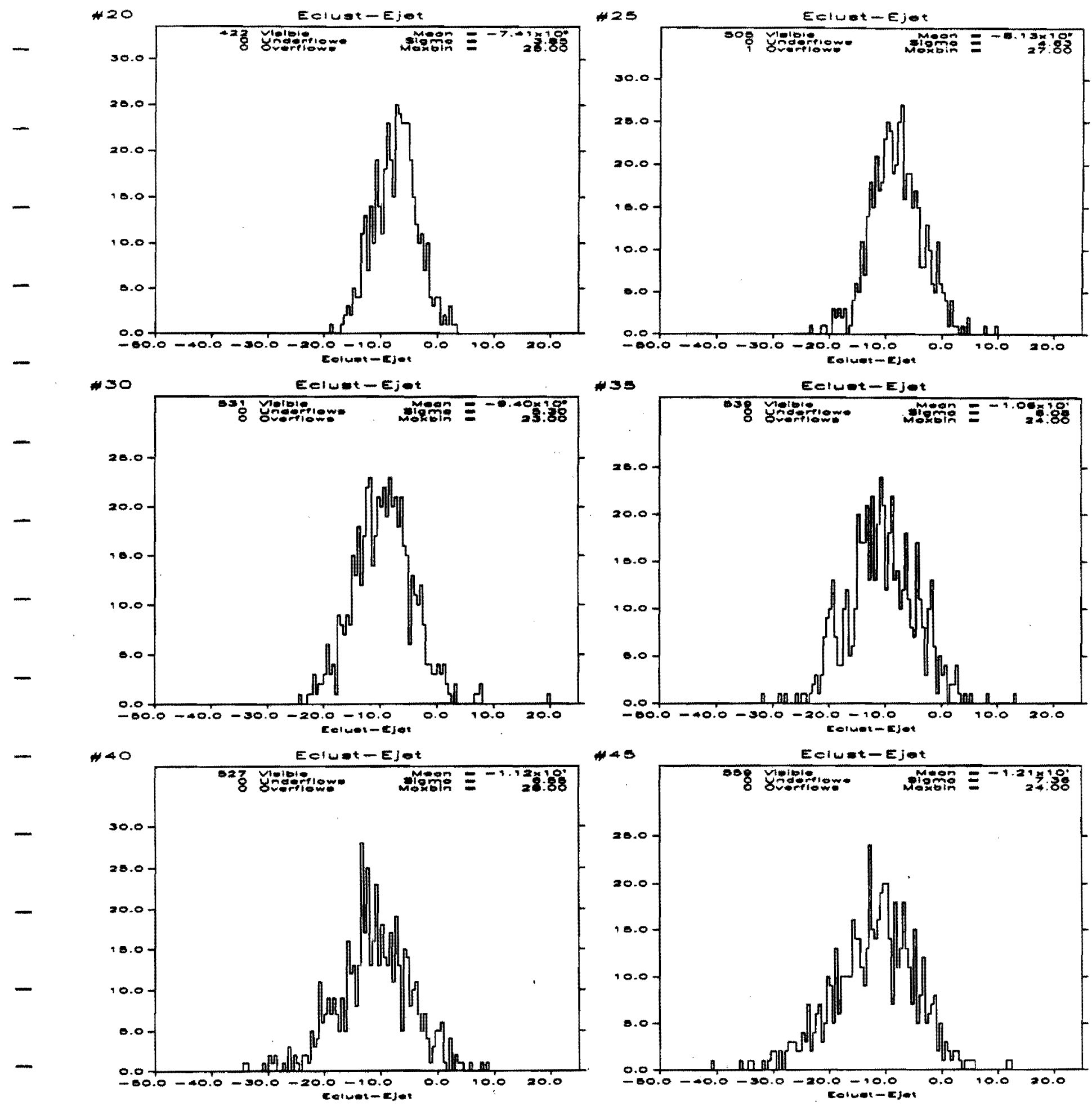

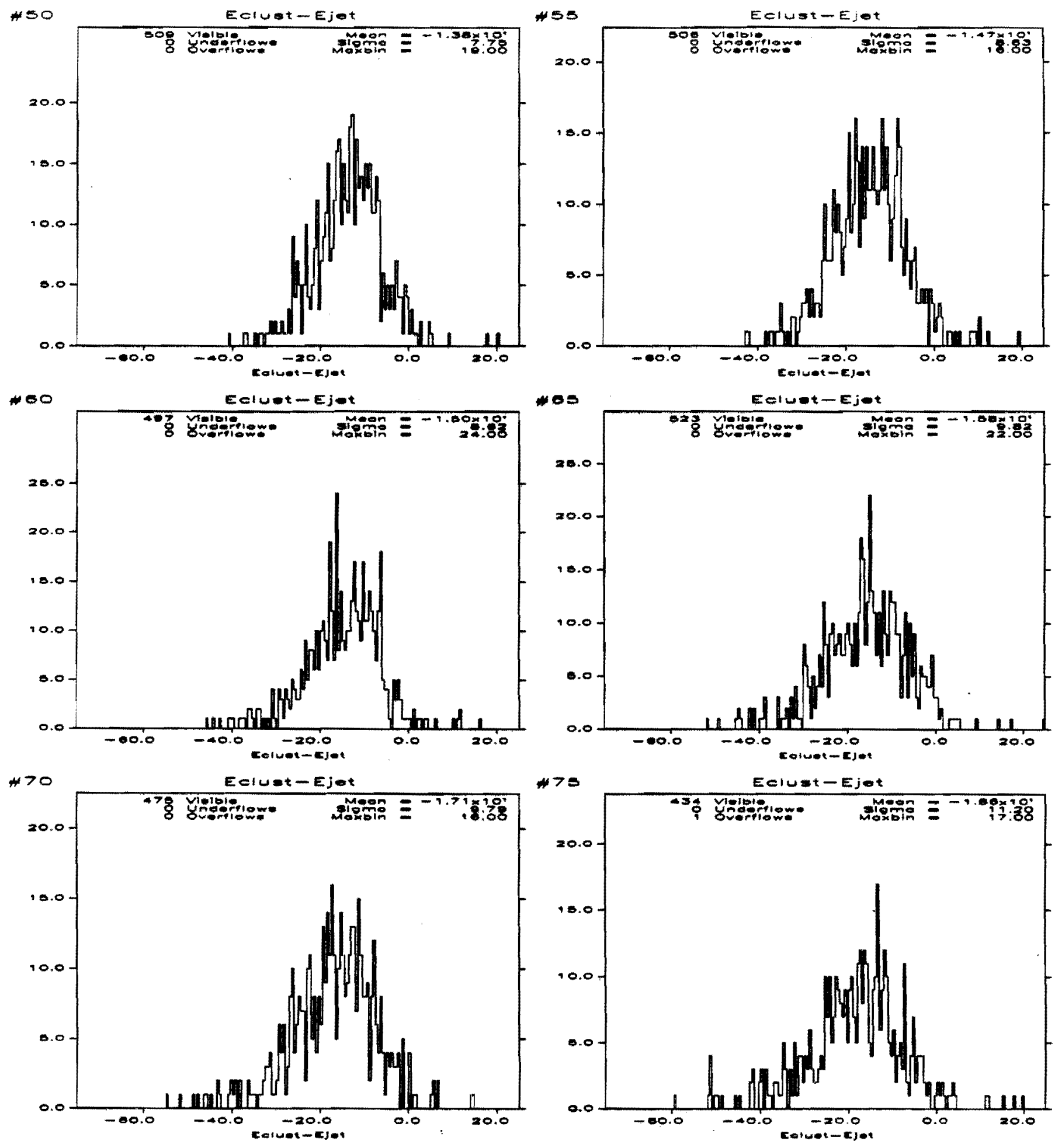

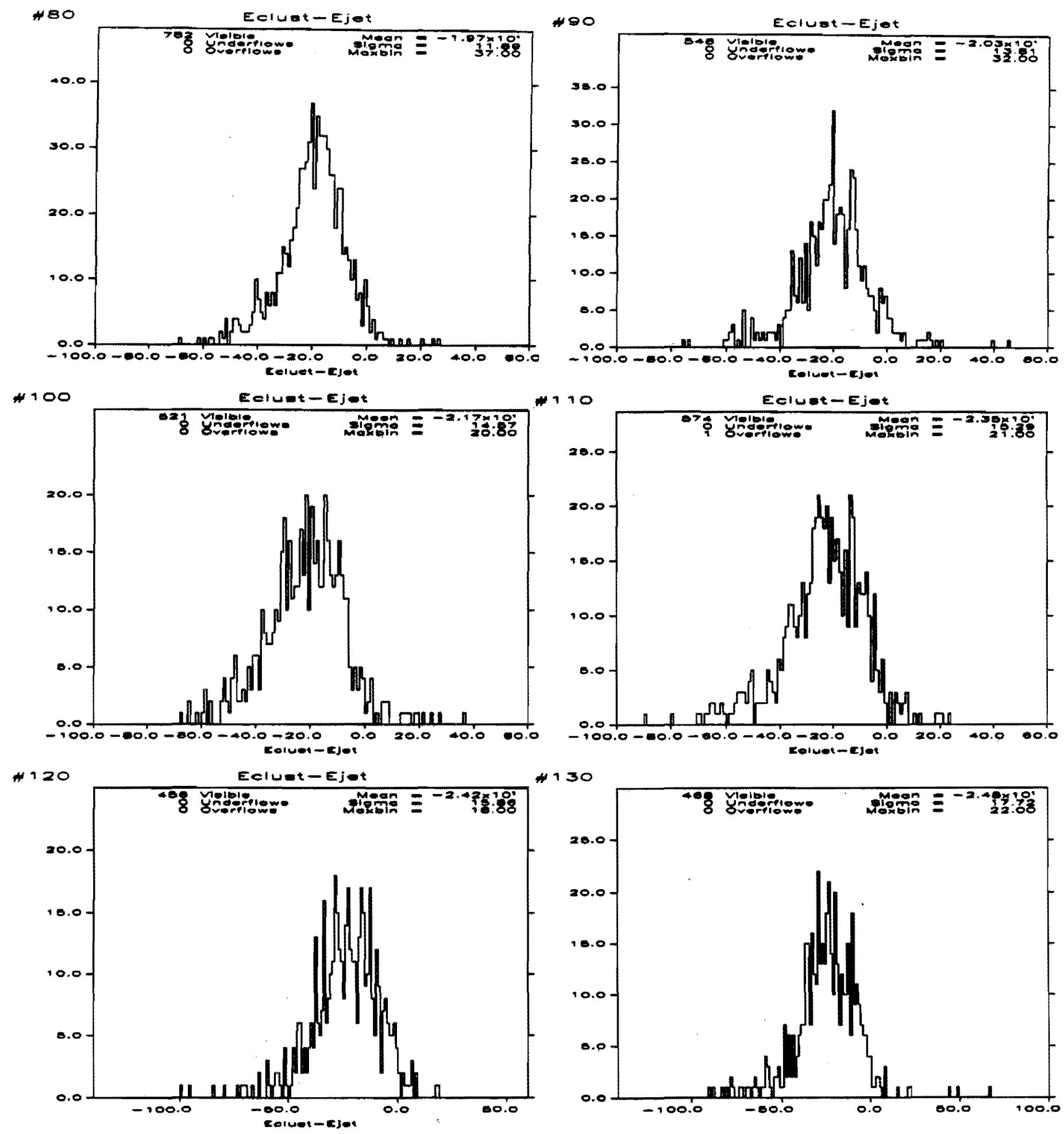

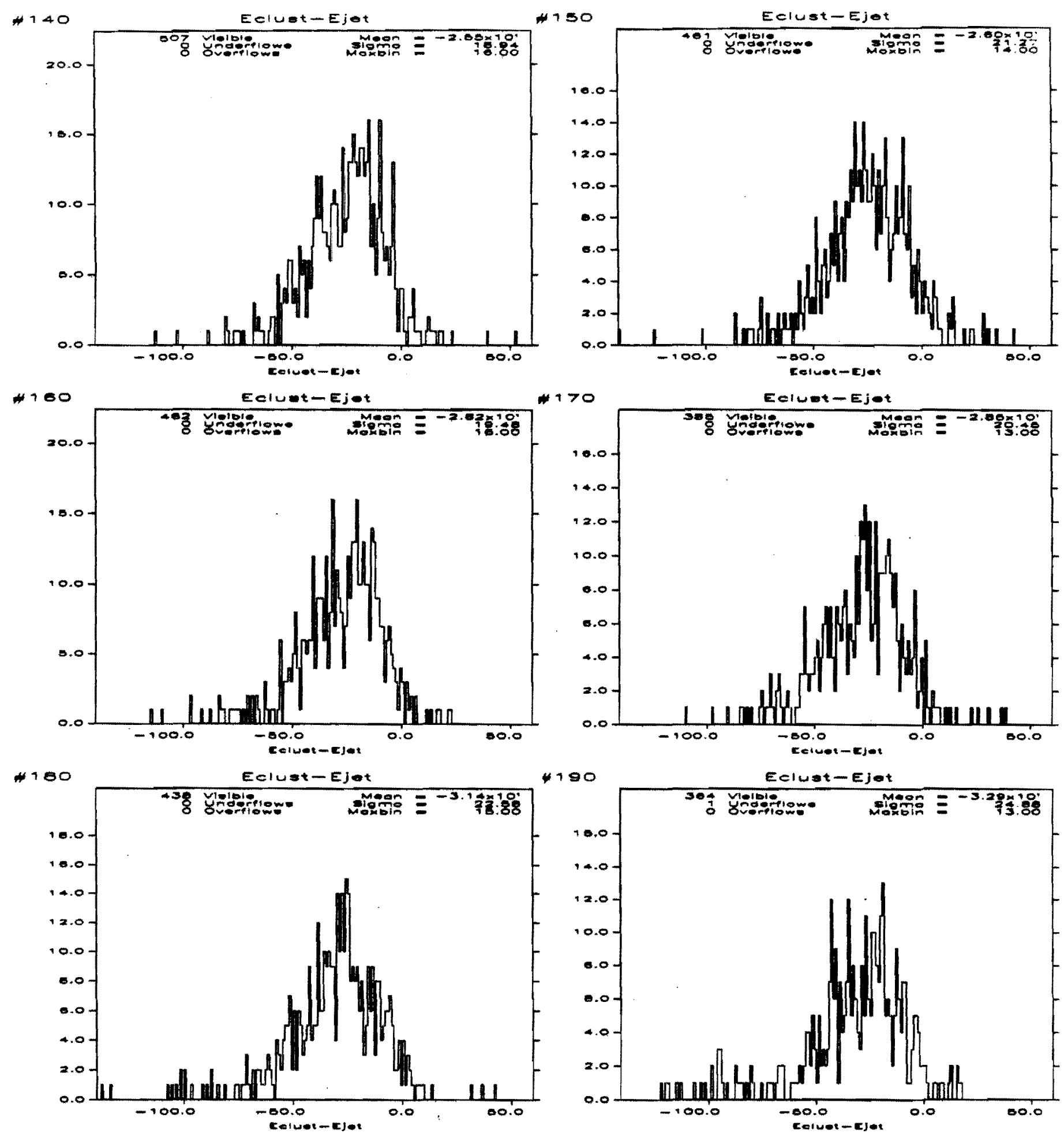


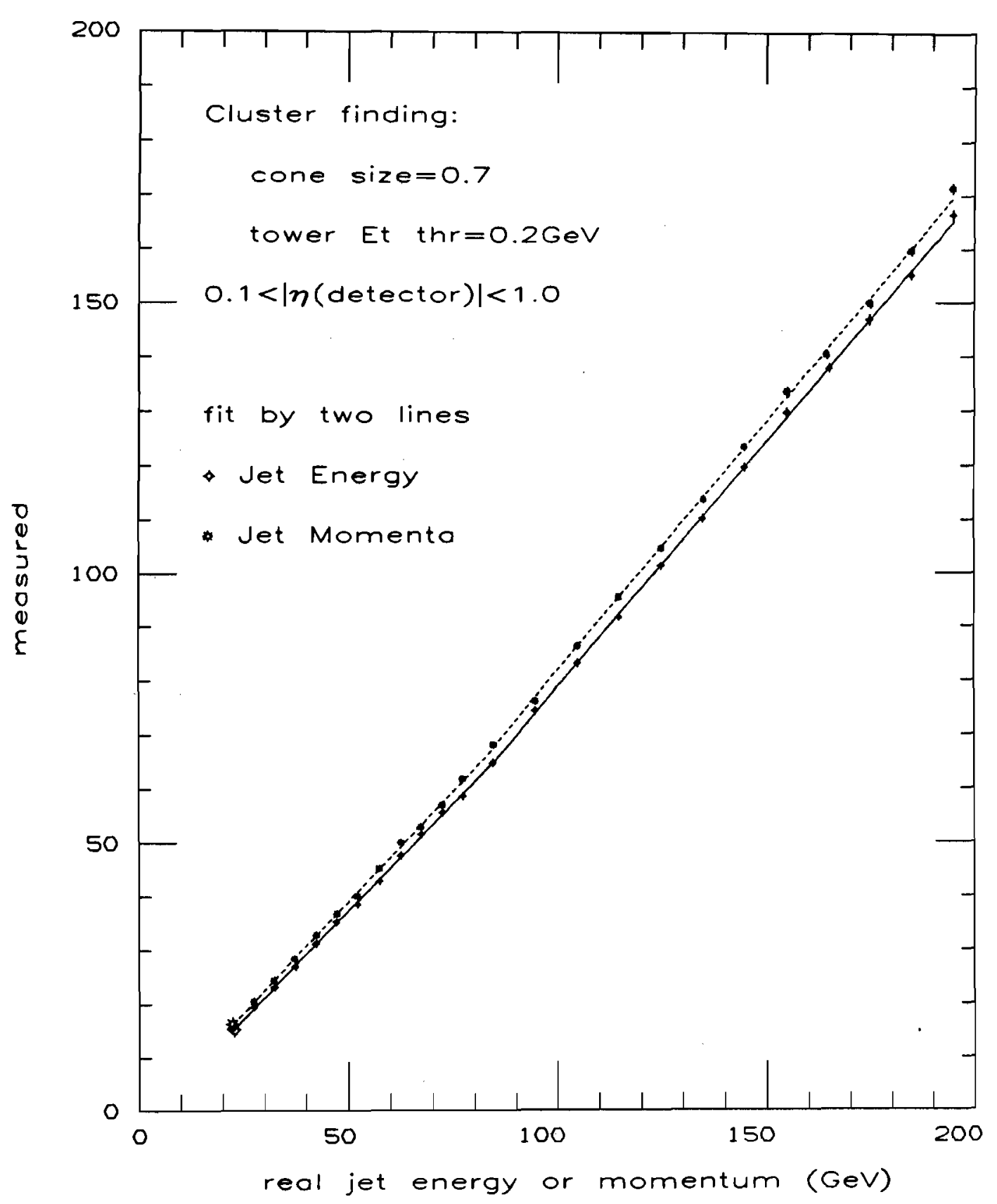

Fig. 50 Measured versus true jet energies \& momenta 


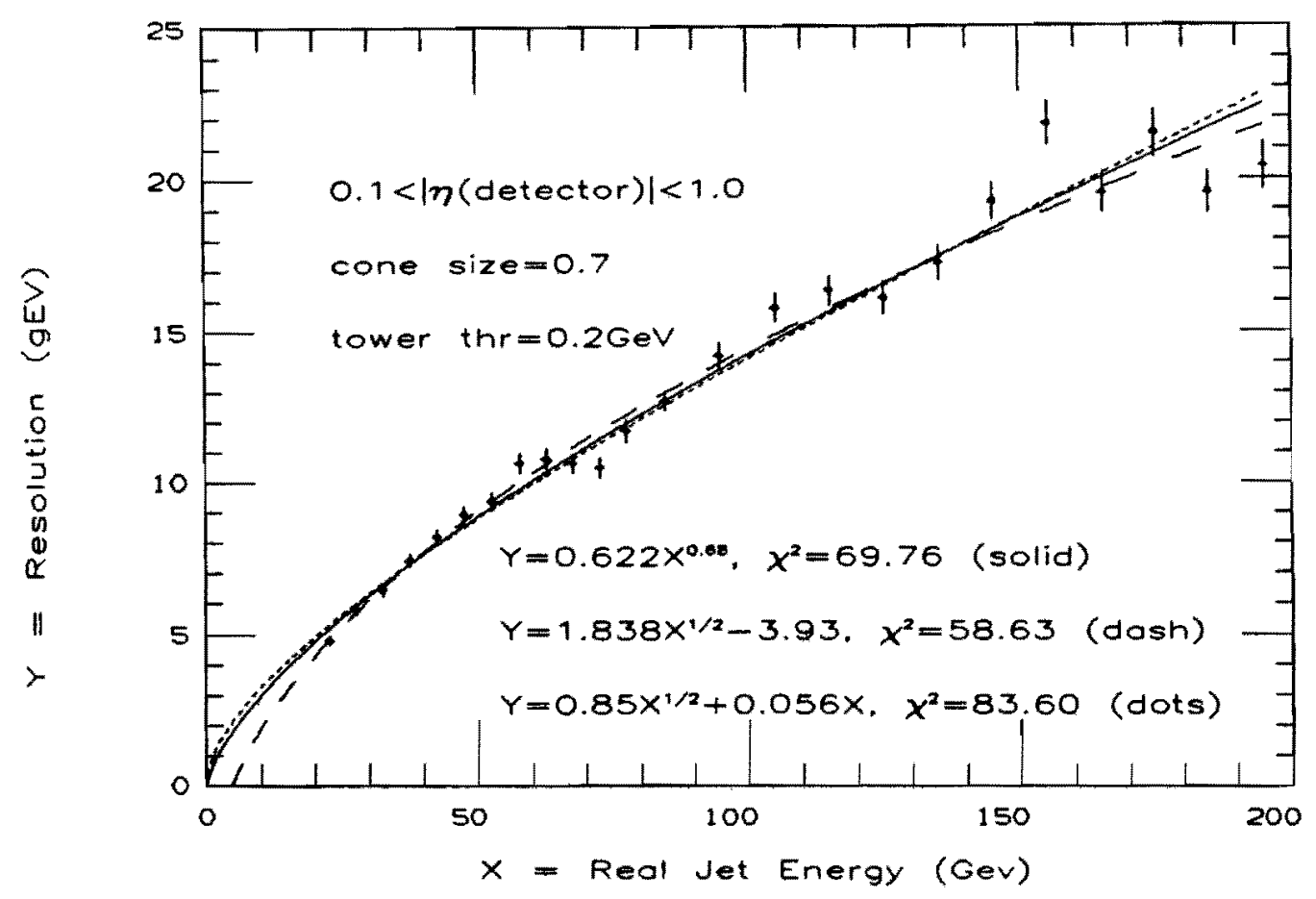

Fig. 51 Jet energy resolution (Monte Corlo)

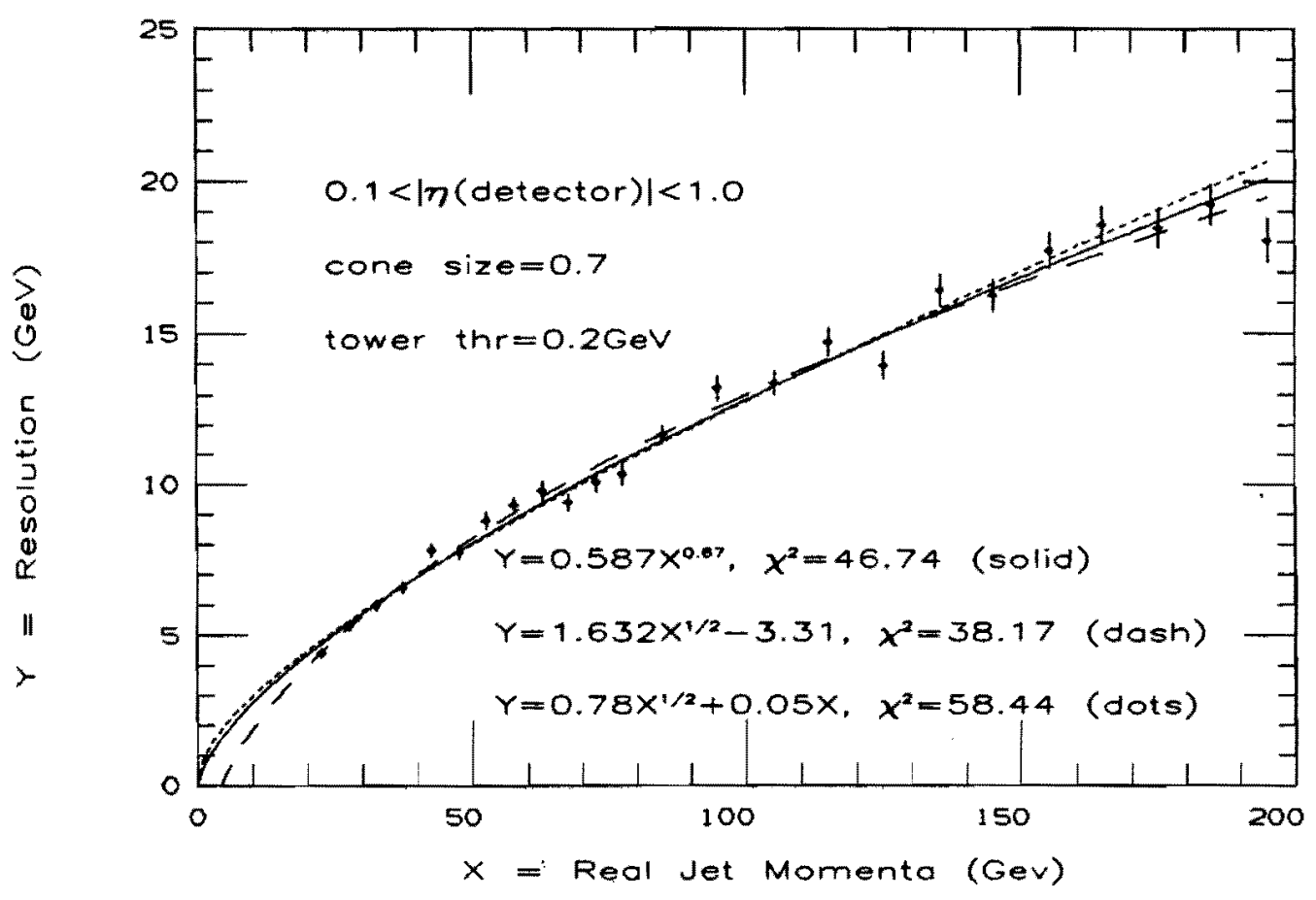

Fig. 52 jet momentum resolution (Monte Carlo) 


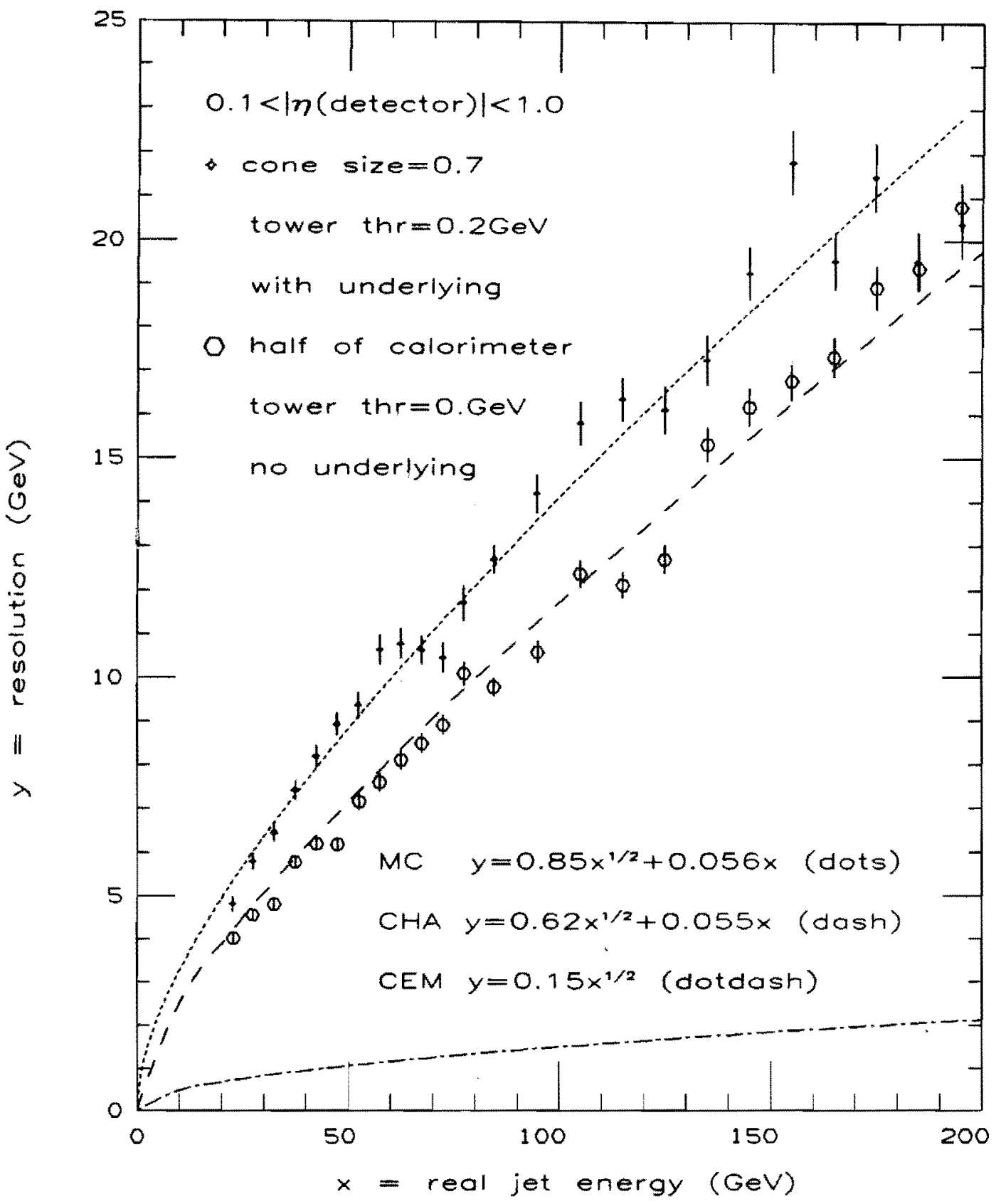

Fig. 53 Monte Carlo jet energy resolutions with or without the effects of clustering and underlying energy. They are compored with the single particle resolutions of CEM and CHA as quoted in the CDF design report. 


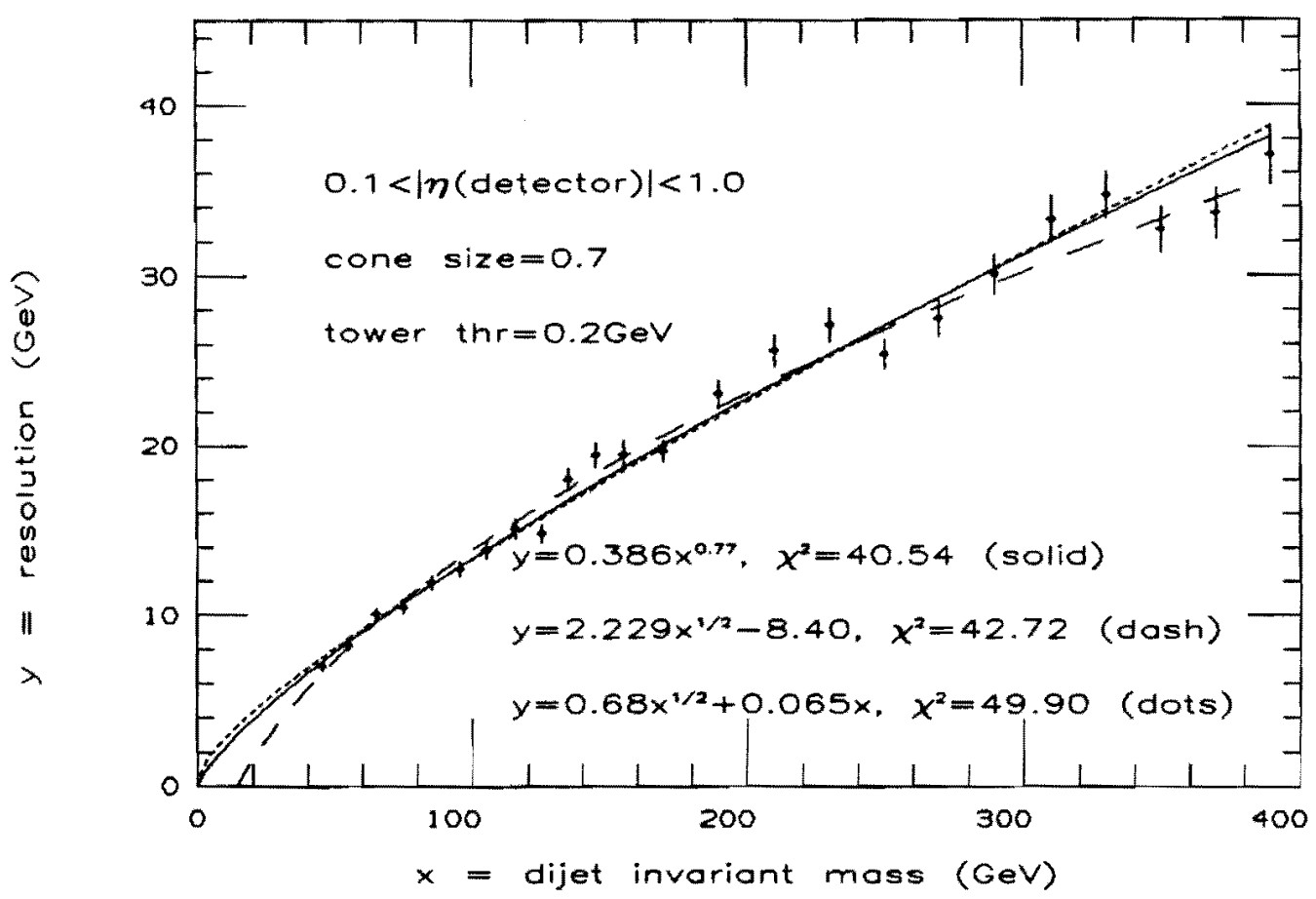

Fig. 54 Dijet mass resolution (Monte Carlo)

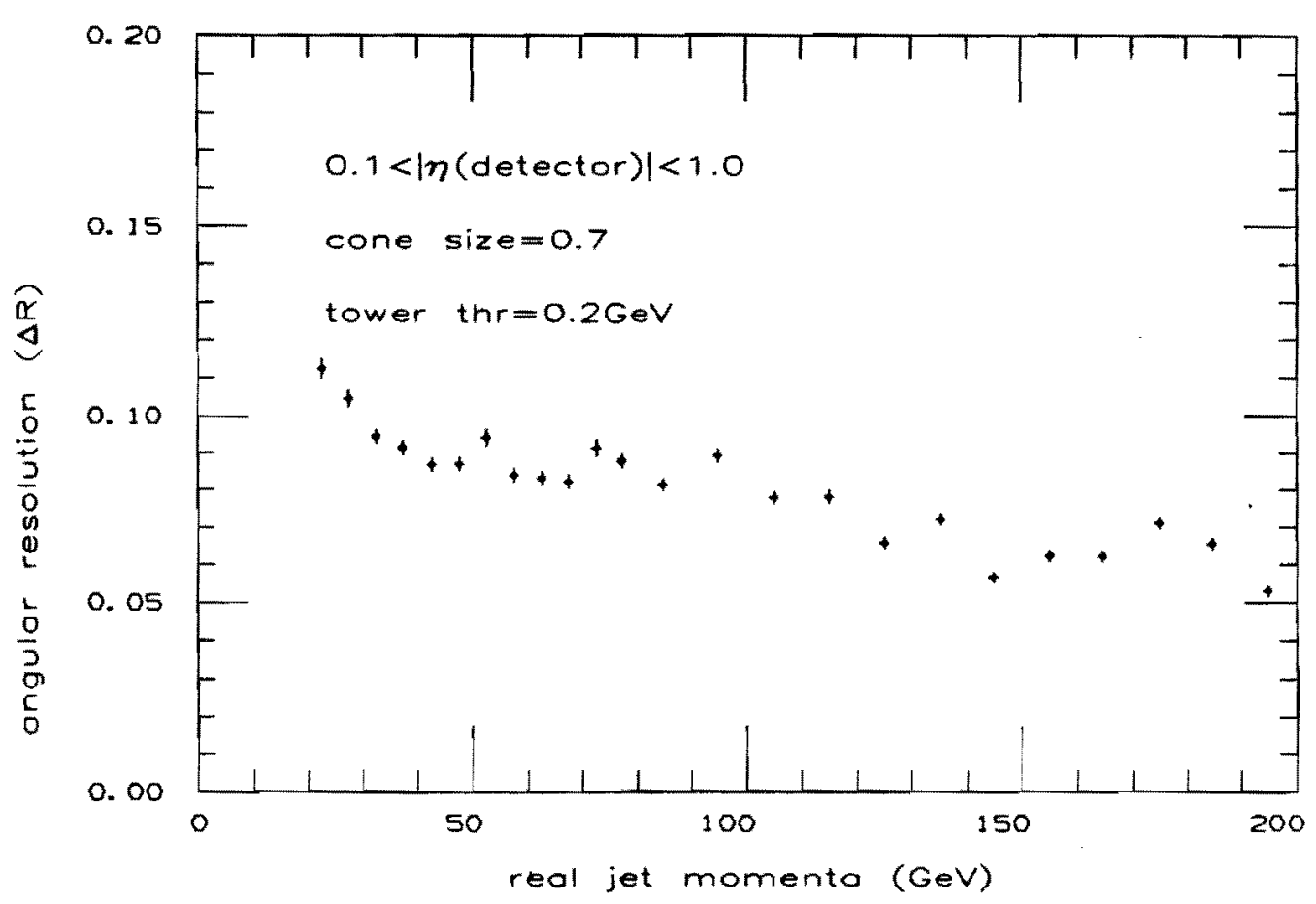

Fig. 55 Jet angular resolution (Monte Carlo) 


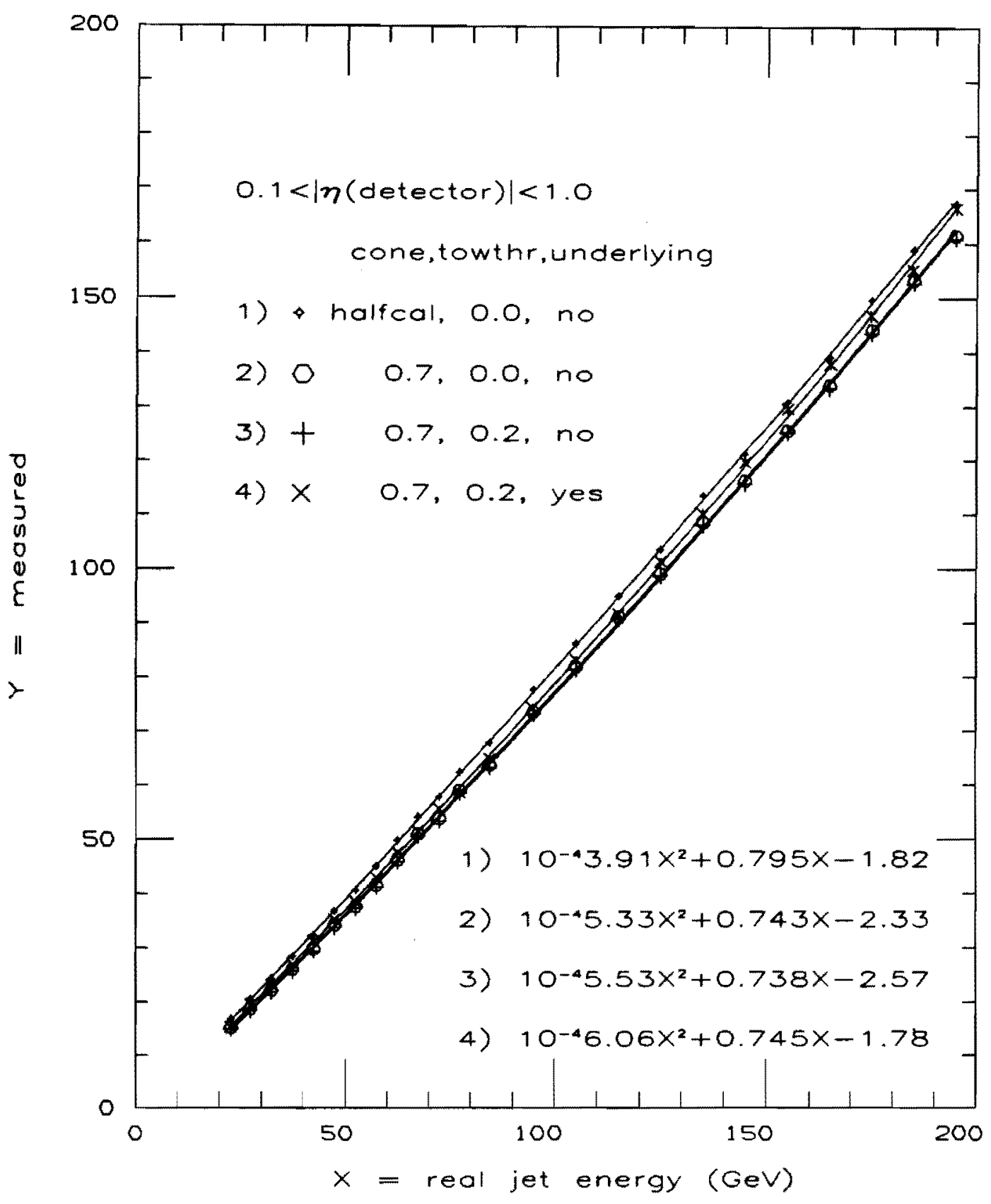

Fig. 56 various ways of collecting colorimetry jet energy. These are used to estimate how energy is lost at each stage. See the text in sect. 7.5 . 


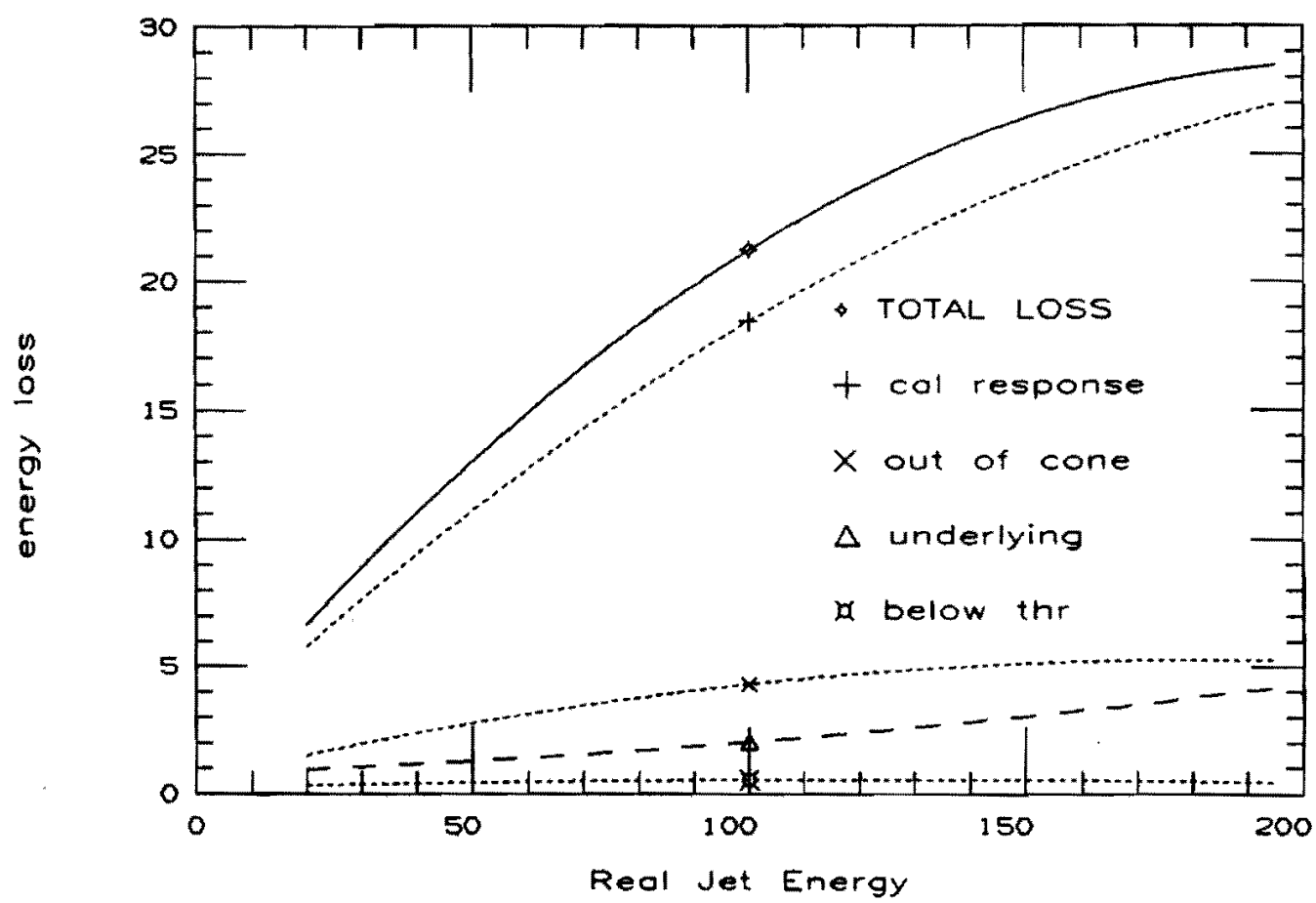

Fig. $57(a)$ Jet energy losses according to Monte Carlo

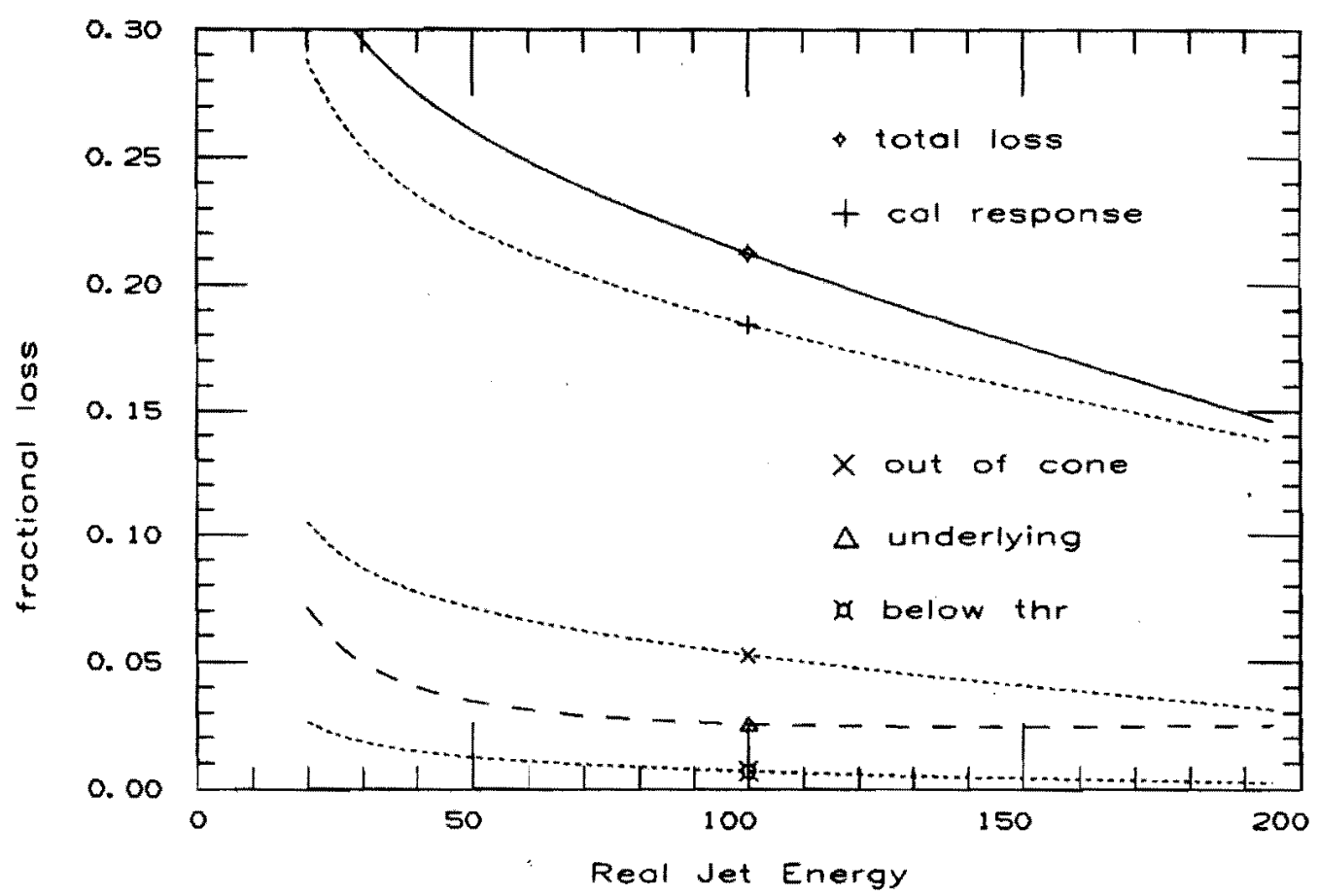

Fig. $57(b)$ Fractional losses of jet energy (MC) 


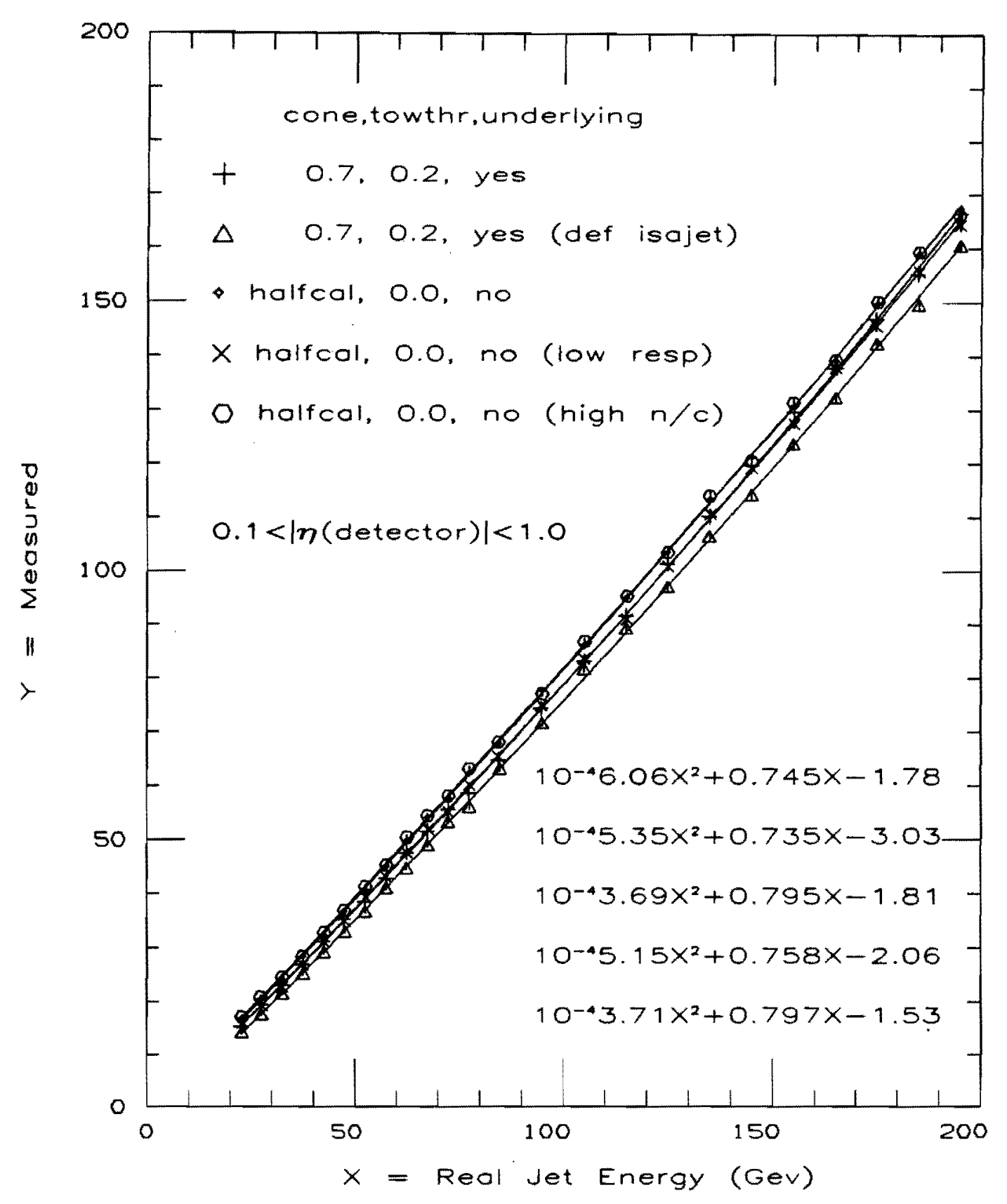

Fig. 58 Measured jet energy when the parton frogmentation and calorimeter response etc. are varied. These are used to estimate the uncertainty in the jet energy correction. 


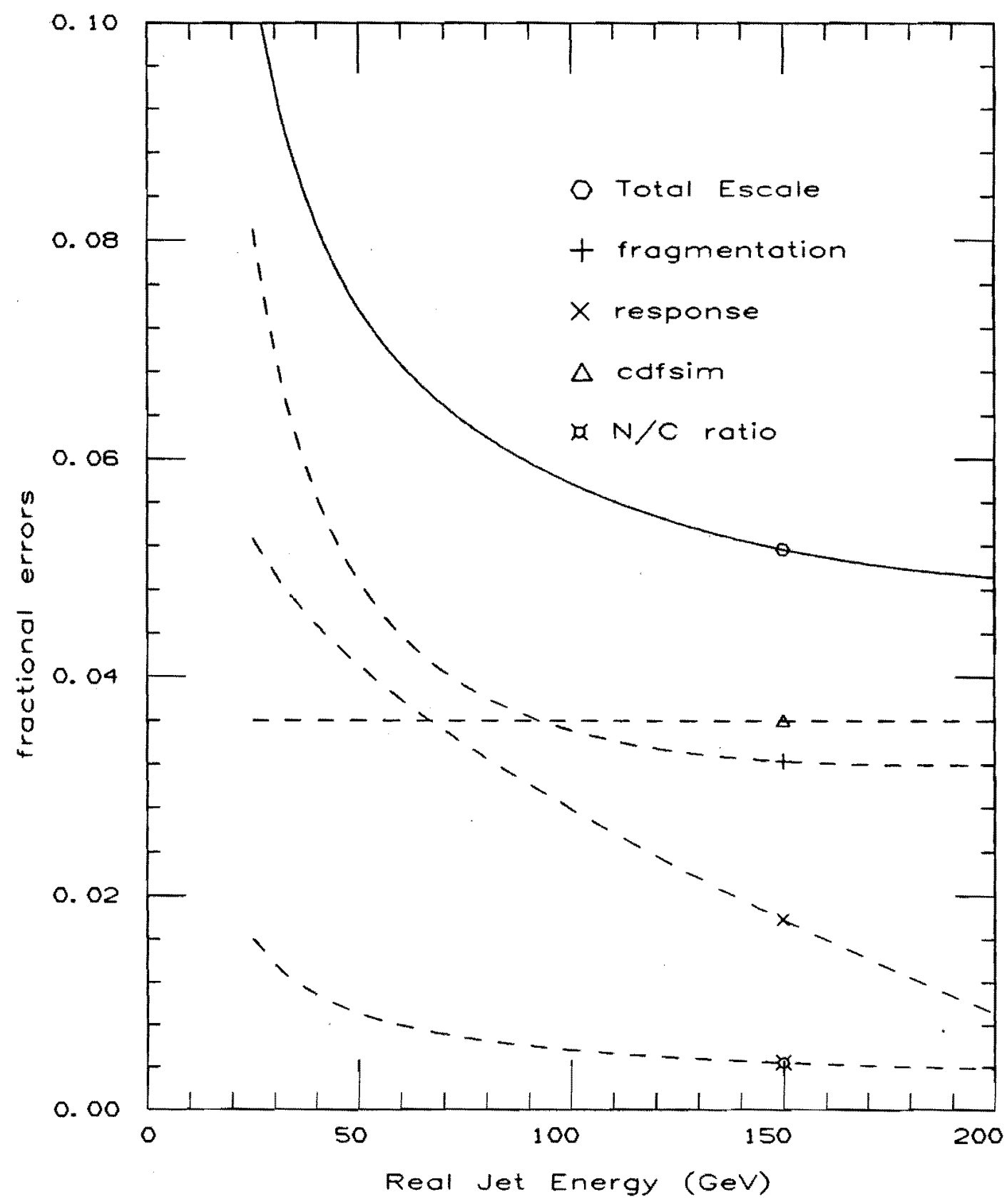

Fig. 59 Uncertainty in the jet energy correction 


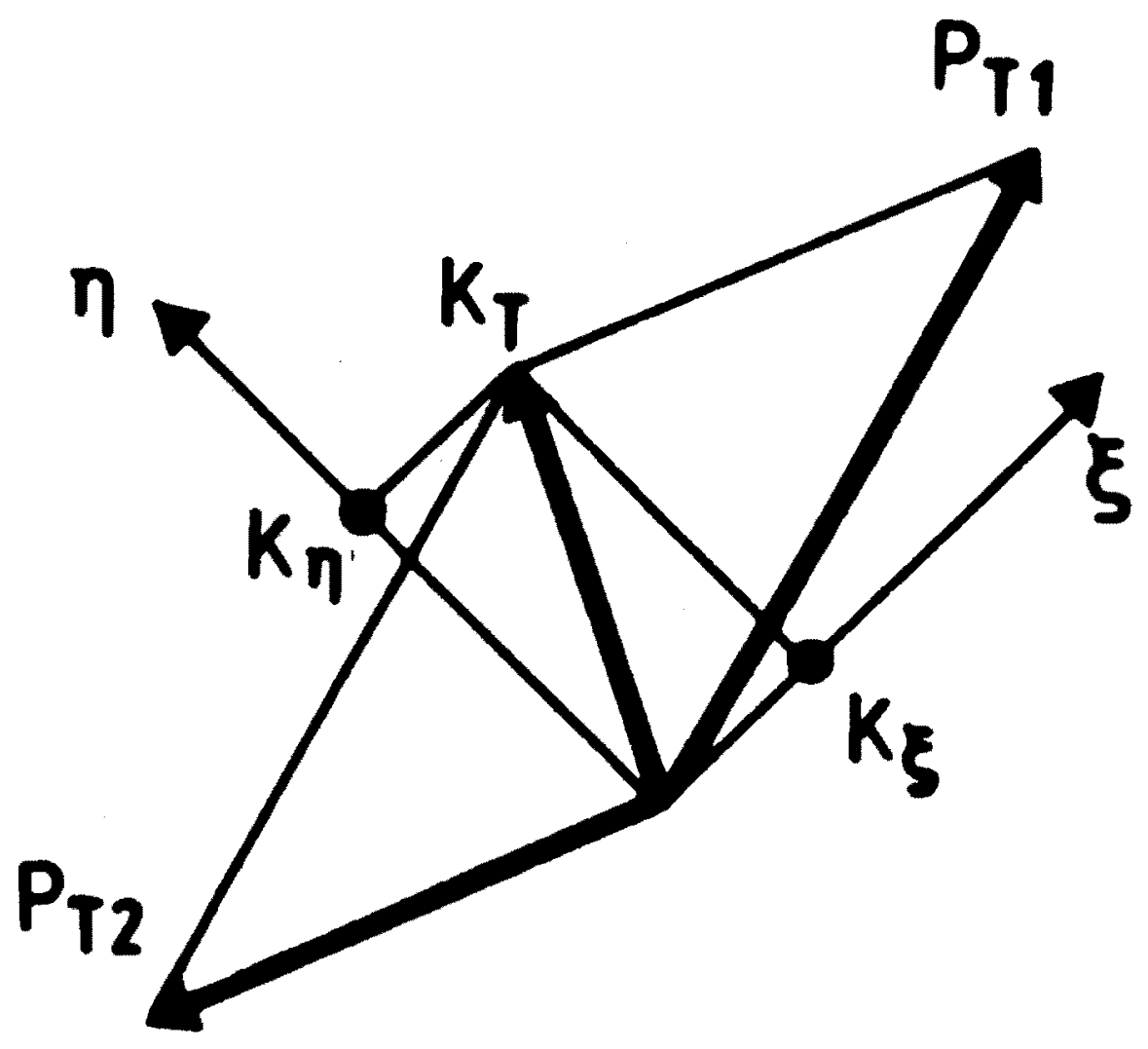

Fig. 60 The definitions of $K_{\$}$ and $K_{\xi}$ in the UA2 Pt balancing technique for determining jet Pt resolution. 


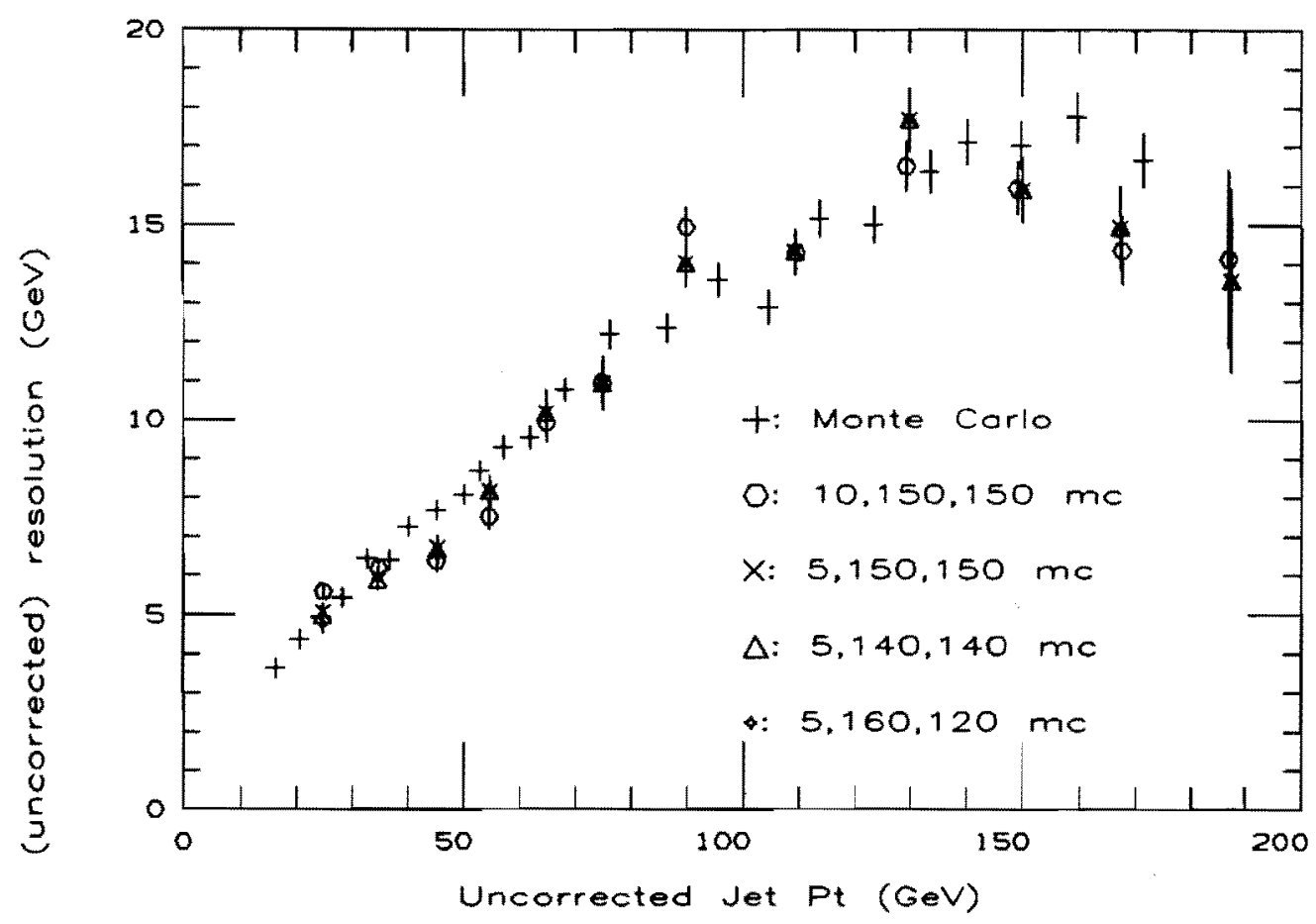

Fig. $61(a)$ Resolution from Monte Carlo data determined by the Pt balance technique with different cuts on the Pt. ( 3rd jet), $\Delta \varphi_{12}$ for $k_{7}$ and $\Delta \varphi_{12}$ for $k_{i}$.

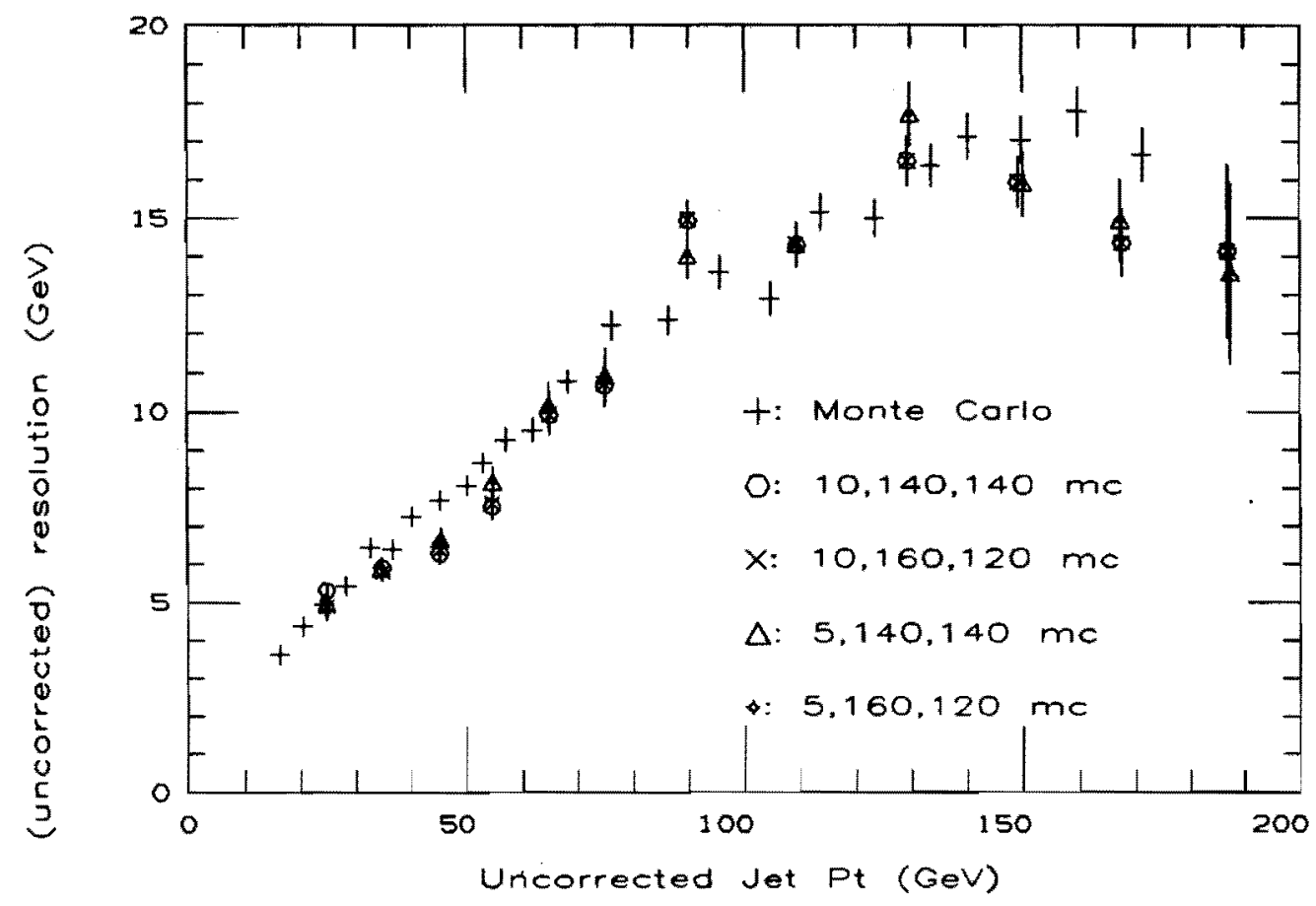

Fig. 61(b) Some os (a) but with different cuts. 


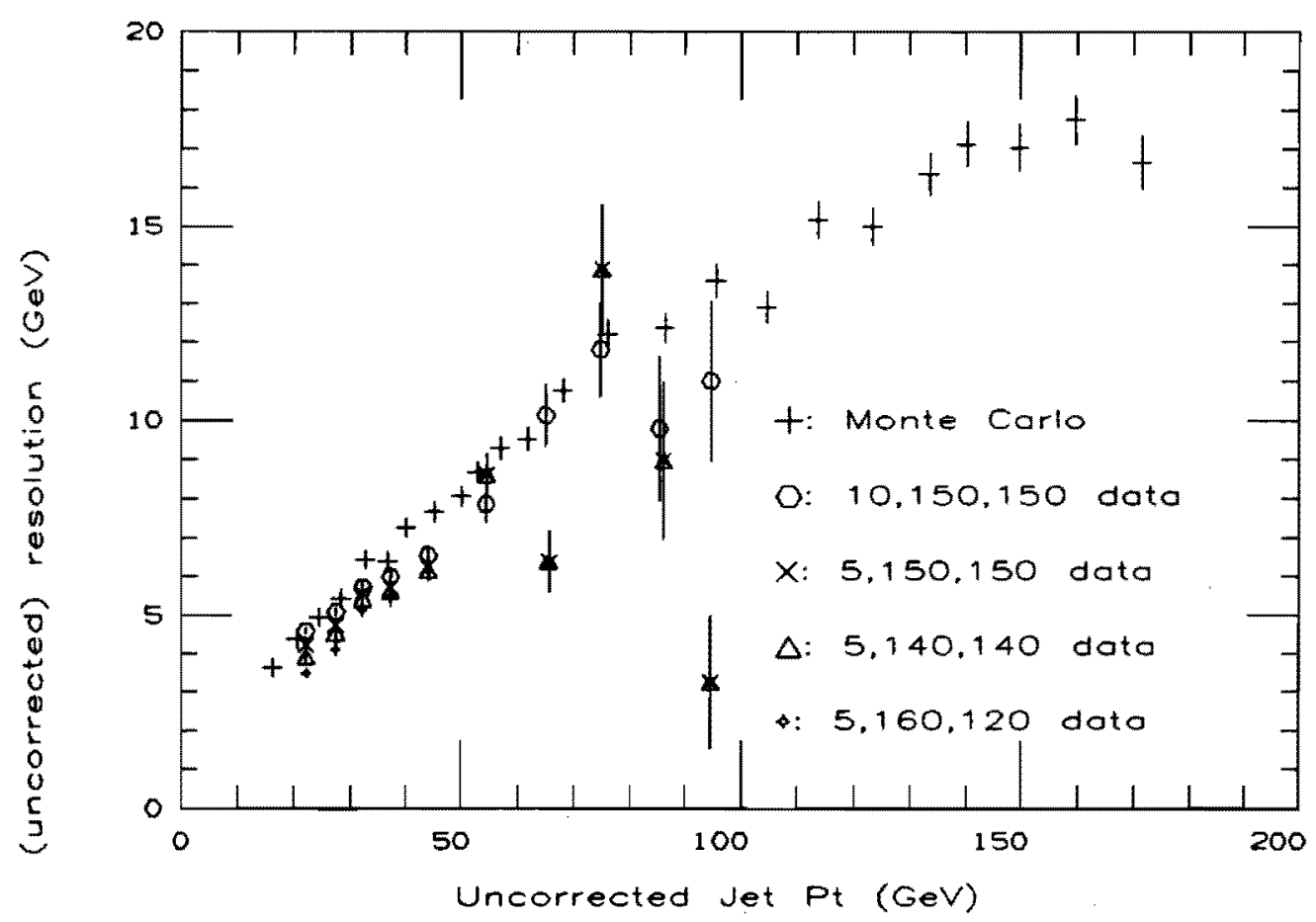

Fig. 62(a) Resolution from real data determined by $\mathrm{Pt}$ balance technique with different cuts on the $\mathrm{Pt}_{3}$ (3rd jet), $\Delta \varphi_{12}$ for $K_{n}$ and $\Delta \varphi_{12}$ for $K_{\text {: }}$

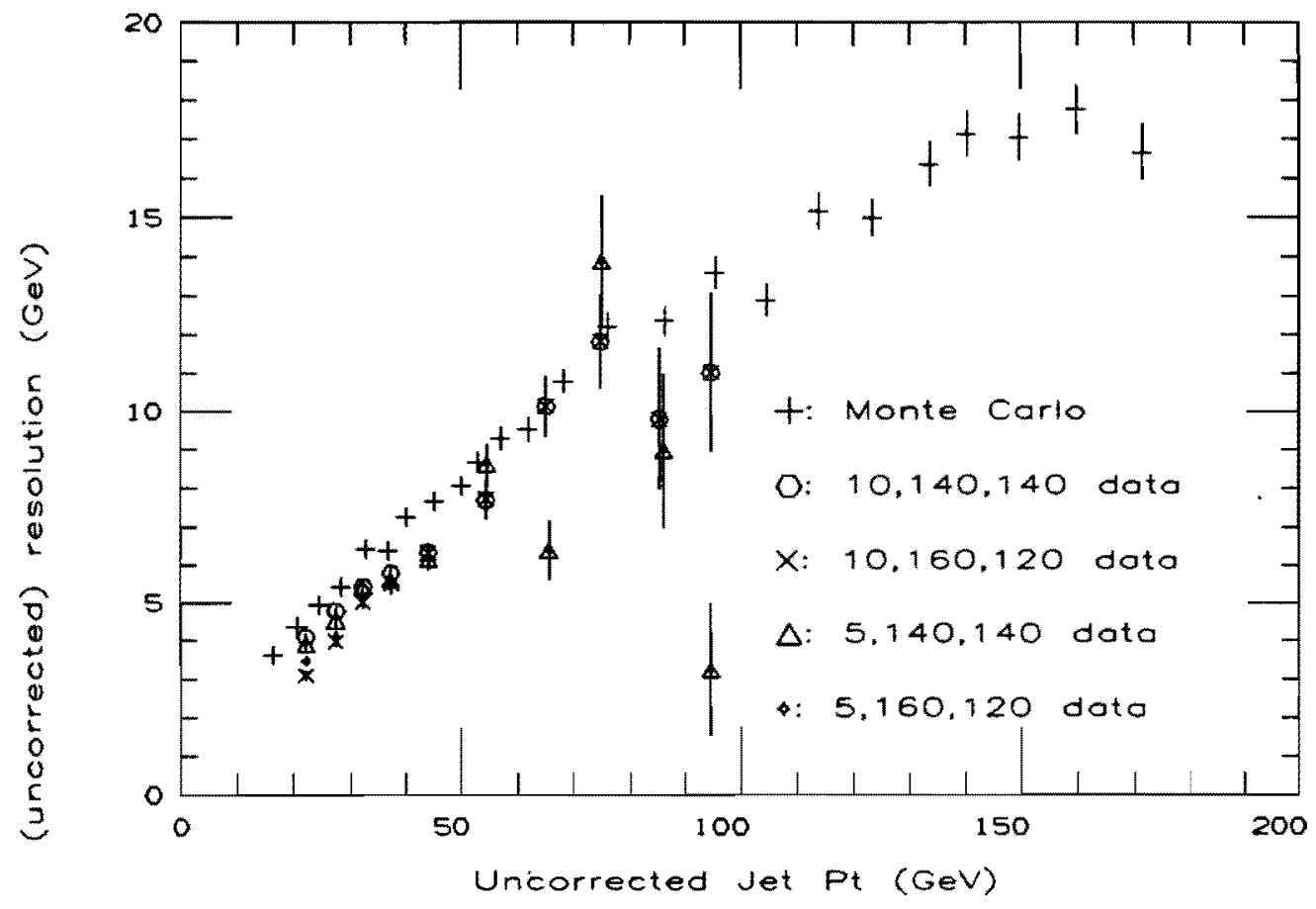

Fig. 62(b) Same as (a) but with different cuts. 


\section{CHAPTER 8}

\section{INCLUSIVE JET ET AND DIJET MASS SPECTRA}

\subsection{Definition Of Central Jets}

The CDF calorimeters cover a pseudo-rapidity ( $\eta$ ) range from -4.2 to 4.2 and has been frequently claimed as a $4 \pi$ detector. But we will restrict ourselves to the central rapidity region for the following reasons: a) The physics in this rapidity range is itself interesting enough; such spectra as $\mathrm{d} \sigma / \mathrm{dPt} / \mathrm{d} \eta$ or $\mathrm{d} \sigma / \mathrm{dM}$ near $\eta=0$ are good tests of $\mathrm{QCD}$; b) We need to know the energy scale with a very high certainty in order to measure spectra which are steeply falling. The central detector has the quality to assure this because it had been carefully calibrated and had stable performance. Furthermore, with the momentum measurement of individual charged particles by the central tracking system, the nonlinearity of the calorimeter response can be checked [45] and used in the Monte Carlo simulation [44] (see Ch. 7); c) Due to several diseases in the gas systems during the 1987 run (the first data run ever), these systems were not armed with triggers and the resulting jet rapidity distribution falls off rapidly near the edge of the central detector. To make it even worse, the energy scale in the gas systems was less than well known because the calibration procedure there was not as precise as that of the central calorimeters.

\subsubsection{Event Vertex Cut}

We then need to define a kinematic range for the "central jets". But let us first decide a cut on the interaction vertices. The vertices that lie far away from the center of the detector $(Z \equiv 0 \mathrm{~cm}$ in the proton beam direction) not only send particles into the calorimeter at funny angles, but also move the jets toward the plug region, where the trigger efficiency is low and the energy scale less certain. In Fig. 63 we plot the vertex

distributions for all events (except Main Ring splash events) from different triggers, and observe a mean of $+3.6 \mathrm{~cm}$ and sigma of $36.7 \mathrm{~cm}$ for 
all triggers combined. If a cut is made at $50 \mathrm{~cm}$, we will lose only about $10 \%$ of events and the data will be healthier. And a $20 \mathrm{~cm}$ outward shift in the vertex will move jets toward the plug region by nearly 0.1 unit of $\eta$ (if $\eta$ is calculated with the radius at the face of hadron calorimeter). So we will cut the vertices at $|Z|=50 \mathrm{~cm}$.

\subsubsection{Pseudo-Rapidity Region}

We now ask the question of a cut on the pseudo-rapidity $\eta$. We want an $\eta$ range as big as possible (for better statistics) but not so large as to suffer from any trigger inefficiency or the inclusion of too much plug energy. The high $Z$ (vertex) events are the most susceptible to end up with a high fraction of plug energy, so we plot the number of jets with a fraction of jet energy higher than $10 \%$ coming from the $p l u g$ calorimeter as a function of jet $\eta$ for $30<Z<50 \mathrm{~cm}$. In Fig. 64 we see the number of such jets rises considerably starting from $\eta-0.7$. The falloff of the jet $\eta$ distribution (Fig. 65) can tell us the $\eta$ beyond which the trigger inefficiency becomes significant. We feel 0.7 is a reasonable place to cut, and this will be our kinematic definition of central jets: $|Z v|<50 \mathrm{~cm}$, and $|\eta|<0.7$.

\subsection{Run Selection and Integrated Luminosity}

The Iuminosity is determined from the Beam-Beam Counters (BBC) east-west coincidence rate assuming a total inelastic scattering cross section of 44 $\mathrm{mb}$ into the $\mathrm{BBC}$ acceptance in $1.8 \mathrm{TeV} \mathrm{p} \overline{\mathrm{p}}$ collisions as extrapolated from SPPS energy [50]. The uncertainty in luminosity is therefore entirely due to the uncertainty (15\%) in this extrapolated cross section. The BBC tracks the good minimum-bias events (as established by event quality criteria using the innermost tracking chamber, the VTPC) to better than $10 \%$.

Not including many special runs intended for various detector studies, the 1987 run collected more than $30 \mathrm{nb}^{-1}$ of data for physics analysis, a remarkable success for a first run. The integrated luminosities and trigger types for these runs are: 


$$
\begin{array}{cl}
\left(\mathrm{nb}^{-1}\right) & \text { (the dominant trigger type) } \\
0.0012 & \text { minimum bias } \\
0.418 & 20 \mathrm{GeV} \text { total central Et threshold } \\
0.143 & 25 \mathrm{GeV} \text { total central Et threshold } \\
17.014 & 30 \mathrm{GeV} \text { total central Et threshold } \\
6.302 & 40 \mathrm{GeV} \text { total central Et threshold } \\
7.492 & 45 \mathrm{GeV} \text { total central Et threshold }
\end{array}
$$

But not all runs are good for use. We will ignore the minimum bias runs because they contribute negligible integrated luminosity and this analysis does not search for minijets. We have checked the quality of each run by its number of Main Ring splash events (Sect. 5.2), mean and width of vertex distribution, jet production cross section and hardware condition. Because each run is a mixture of several parallel triggers, we first select events that satisfied the total Et trigger (according to a trigger bit). These selected events were then processed with full cleanup (see Ch. 5) and standard CDF jet analysis (with the JETCLU algorithm, Sect. 6.1.3). Some quantities showing the features of each physics run are listed in Appendix $F$, where we recommend the exclusion of several runs marked by *'s before the run numbers. Runs before 7214 are not included for use because no gain constants were loaded into the hardware during the early period of run (3.41 $\left.\mathrm{nb}^{-1}\right)$. Other runs are rejected mostly due to a large fraction of Main Ring splash events. Numbers that call our attention for further investigation (because of a deviation from the average behavior of other runs) are also marked by '*', but the runs are not necessarily rejected because with such a low number of events the deviation (e.g. mean or width of the vertex $Z$ distribution, or the jet cross section) may be not significant. But two runs were actually rejected because of an extremely low number of central jet events and one was rejected with additional trigger problems (many missing events). Several runs were rejected for having a very low number of events, indicating some troubles encountered during the run; furthermore with such a low number of events the luminosity calculation is not very reliable. We finally end up with the following samples: 


$\begin{array}{rl}\left(n b^{-1}\right) & \text { (the dominant trigger type) } \\ 0.418 & 20 \mathrm{GeV} \text { total Et threshold } \\ 13.224 & 30 \mathrm{GeV} \text { total Et threshold } \\ 6.139 & 40 \mathrm{GeV} \text { total Et threshold } \\ 6.466 & 45 \mathrm{GeV} \text { total Et threshold }\end{array}$

We have rejected $4.265 \mathrm{nb}^{-1}$ of data and another $0.856 \mathrm{nb}^{-1}$ was lost due to magnetic tape errors, and there remains $26.25 \mathrm{nb}^{-1}$.

These integrated luminosities must be corrected for the vertex cut we decided in Sect. 8.1.1. With a cut at $|Z|=50 \mathrm{~cm}$, the correction factors for these four trigger samples are $0.800,0.824,0.855$ and 0.869 respectively. The total effective integrated luminosity is 22.1 . The width of the vertex distribution (Fig. 63) is found to decrease with Et threshold. This might be due to the fact that the trigger hardware assumes $Z=0 \mathrm{~cm}$ and tends to under-calculate Et's for events with vertices far from $Z=0 \mathrm{~cm}$.

\subsection{Criteria for Good Clusters}

Although the hadron TDC filter rejects more than $99 \%$ of the Main Ring splashes, still about $5 \%$ of cosmic rays will creep into the $35 \mathrm{nsec}$ in-time window (out of $700 \mathrm{nsec}$ ). The spectrum for these cosmic rays as seen by the CDF central calorimeters is plotted in Fig. 66. A powerful measure to identify these extra terrestrials is to cut on the electromagnetic to total energy ratio (EM/TOT) because almost all cosmic rays deposit purely hadronic or sometimes purely electromagnetic energy with only very small number of towers involved. The distributions of the EM/TOT for jets and for cosmic rays with uncorrected Et above $30 \mathrm{GeV}$ are plotted in Fig. 67. From these plots we have opted to cut out the clusters with ratios in the lower and upper $5 \%$ regions (containing $2 \%$ of jets). A complete scanning of all high Pt clusters ( $>100 \mathrm{GeV}$ ) showed this cut essentially removes all cosmic rays. This cut also removes isolated high $\mathrm{Pt}$ electrons, photons or spurious hot tube energy that has escaped the single-tube suppressor (Sect. 5.1).

Another possibility is to cut on the missing Et of the event (Fig. 68), Large missing Et can result from real physics such as $Z+$ jet followed by $Z$ 
$\rightarrow \nu \nu$, new phenomena such as supersymmetry, or simply from cosmic rays or mismeasurement of jet energy. We have found by scanning that some real high Pt events failed the missing Et cut because one of jets was badly measured. Since we already have a very efficient EM/TOT cut, we feel there is no need for the missing Et cut.

\subsection{The Inclusive Jet Et and Dijet Mass Spectra}

We will make two plots for central jet production: an inclusive jet Et distribution and a dijet invariant mass distribution. The definition of central jets has been given in Sect. 8.1. The dijet mass is defined as the following. We require the two highest $\mathrm{Pt}$ jets in the whole event (including jets in the forward gas systems) to be in the region $-0.7<\eta<0.7$, and the dijet invariant mass is calculated from the corrected energy and momenta by the formula

$$
M_{J J}=\sqrt{\left(E_{1}+E_{2}\right)^{2}-\left(P_{1}+P_{2}\right)^{2}}
$$

All jet data were taken with certain total Et thresholds and thus suffer from trigger inefficiency for low $\mathrm{Pt}$ or $M_{J J}$ regions. Mixing up these samples without careful jet selection will put us in a difficult, though not impossible, situation on how to correct for the inefficiencies. But we can use a lower threshold sample to determine the inefficiency region of a higher threshold sample by comparing their spectra and finding the point where the two spectra meet, assuming the lower threshold sample is fully efficient at the meeting point. From Figs. 69 - 70 we have chosen to use only those jets with Et greater than $[30,45,60,65] \mathrm{GeV}$ or dijet mass greater than $[60,60,90,90] \mathrm{GeV}$ in the samples taken with trigger thresholds $[20,30,40,45] \mathrm{GeV}$ respectively. To obtain the full spectra, we collect all jets (or dijet events) above these thresholds after applying all cuts mentioned in the previous sections (see Tables $10-11$ ) and divide the number of jets (or events) by the appropriate integrated luminosities (the sums of vertex-corrected integrated luminosities of all contributing samples). We have chosen a fixed bin size of 5 (10) GeV for the low Et (low dijet mass) part of the spectrum and used variable bin sizes, increasing 
roughly with the detector resolution, for the high Et (mass) region where the number of events is low. In fact, the binnings have been carefully planned such that the bin-to-bin variations, which are not due to real physics, are minimized. The justification for this is that any real physical bumps must be wider than our detector resolution.

\subsubsection{Issue of Resolution Smearing}

Given a real spectrum $f(E)$, the measured spectrum f'(E) by a detector with Gaussian resolution $\sigma(E)$ is given by

$$
f^{\prime}(E)=\int_{0}^{\infty} \frac{d \epsilon}{\sqrt{2 \pi}} \frac{1}{\sigma(\epsilon)} \exp [-(E-\epsilon) / 2 \sigma(\epsilon)\rfloor f(\epsilon)
$$

If $f(E)$ is steeply falling, and $\sigma(E)$ is not increasing too fast, then at any $E$ the flowing from lower $E$ to higher $E$ is bigger than that from higher $E$ to lower $E$, and the effect of the resolution smearing is to raise the spectrum up, i.e. $f^{\prime}(E)>f(E)$.

There are two ways to compare our measurement with theory. One is to smear the theoretical spectrum by a known resolution and compare it to the one observed. Or we can correct each data bin by a unsmearing factor and compare the unsmeared spectrum to the theory. The second approach is probably preferred by most people because detector resolution is an experimental issue and shouldn't be worried about by theorists. But in either approach, knowing the resolution function $\sigma(E)$ is vital. In $\mathrm{Ch} 7 \mathrm{We}$ determined the $\sigma(P)$ both by Monte Carlo simulation and by a Pt balancing technique invented by UA2. We were not conclusive on whether these two determinations agree with each other because the UA2 technique depends very much on the dijet selection (for our 1987 data). One dijet selection did give very good agreement with Monte Carlo, but other selections had resolution lower by up to $20 \%$. Our 1987 data did not allow us to go too far in $\mathrm{Pt}(\mathrm{e.g.}>70 \mathrm{GeV})$, thus there is yet no comparison for the high Pt jets. Nonetheless, we decide to go with Monte Carlo resolutions for all of the jet energy, momenta and dijet masses and include in the systematic errors the fact that these $\sigma$ 's may be $20 \%$ off from the real values.

Our unsmearing procedure is conceptually very simple. A chosen function is smeared with the assumed energy-dependent Gaussian resolution, and then 
is fitted to the measured spectrum. The correction factors are then derived from the ratio of the original and the smeared functions. The $\chi^{2}$ from the fit is calculated from the number of jets (events) observed and the number predicted using Poisson statistics, where the predicted number is obtained by integrating the smeared function over the bin range. We have used the functional form $f(E)=A(E+a)^{-\beta}$ for the jet Et spectrum and $g(m)=A m^{-}$ $a_{\exp (-\beta m)}$ for the dijet mass spectrum. In these cases $\alpha$ and $\beta$ are varied to minimize the $\chi^{2}$ while $A$ is merely the normalization factor. The results are given in Table $12-13$ (and Figs. 71 - 72).

But we must note that this procedure of unsmearing the spectrum is good only if the measured or theoretical spectrum (e.g. standard QCD) is really as structureless as the function we have assumed, for in this case the resolution unsmearing amounts to moving down the measured spectrum without changing its shape (except slowly). But for models involving bumps or unusual tails, the correct procedure is to compare the measured spectrum and the smeared theoretical spectrum directly, as we will see in Sect. 9.3 where we compare the dijet mass spectrum with a theory involving the socalled axigluon.

\subsubsection{The Systematic Uncertainties}

We have discussed the systematic uncertainty in the jet energy correction and resolution in $\mathrm{Ch}$. 7. There are uncertainties due to the jet fragmentation model, the charged-to-neutral particle ratio, the nonlinear response of the calorimeter, and the goodness of the detector simulation. These uncertainties are translated into uncertainties in cross sections via the formula: $\Delta f(E)=f(E)-f(E-\Delta E)$, where $f(E)$ is a best fit to the data (Sect. 8.4.1). In addition to these, there is a $15 \%$ uncertainty in the luminosity measurement and $3 \%$ in the acceptance correction for the vertex cut. The various systematic errors in the cross sections are plotted in Fig 73 - 74 and the final spectra are given in Tables 14 - 16 (and Figs. 77 79). The Et (or dijet mass) value for each bin is determined through $f(E)=$ $\int f(\epsilon) d \epsilon / \Delta$, where the integration is over the bin range and $\Delta \equiv$ the bin width. This $E$ can be very different from the mean Et (dijet mass) of the observed jets (events) in the bin. 
Table 10 Number of jets that survived each cut in turn (Et spectrum).

\begin{tabular}{|c|c|c|c|c|c|}
\hline & \multicolumn{4}{|c|}{ trigger total Et threshold } & \multirow{2}{*}{ cuts applied } \\
\hline & $20 \mathrm{GeV}$ & $30 \mathrm{GeV}$ & $40 \mathrm{GeV}$ & $45 \mathrm{GeV}$ & \\
\hline 0) ${ }^{*}$ & 23450 & 237983 & 40535 & 27280 & $|Z|<50 \mathrm{~cm},-10<t<25 \mathrm{~ns}$ \\
\hline \multirow[t]{2}{*}{ 1) } & 1970 & 42794 & 10197 & 7644 & $|\eta|\langle 0.7, \mathrm{Et}\rangle 30 \mathrm{GeV}$ \\
\hline & $8.4 \%$ & $18.0 \%$ & $25.2 \%$ & $28.0 \%$ & \\
\hline \multirow[t]{2}{*}{ 2) } & 1958 & 42614 & 10139 & 7615 & $0.05<E M F<0.95$ \\
\hline & $99.4 \%$ & $99.6 \%$ & $99.4 \%$ & $99.6 \%$ & \\
\hline \multirow[t]{2}{*}{ 3) } & 1958 & 11383 & 1332 & 1024 & Et) $30,45,60,65 \mathrm{GeV}$ \\
\hline & $100 \%$ & $26.7 \%$ & $13.1 \%$ & $13.4 \%$ & \\
\hline \multirow[t]{2}{*}{ 4) } & 1939 & 11362 & 1330 & 1023 & $\#$ of clusters > 1 \\
\hline & $99.0 \%$ & $99.8 \%$ & $99.85 \%$ & $99.9 \%$ & \\
\hline
\end{tabular}

Table 11 Number of events that survived each cut in turn $\left(M_{J J}\right)$.

\begin{tabular}{|c|c|c|c|c|c|}
\hline & \multicolumn{4}{|c|}{ trigger total Et threshold } & \multirow{2}{*}{$\begin{array}{l}\text { cuts applied to } \\
\text { the two highest Pt jets }\end{array}$} \\
\hline & $20 \mathrm{GeV}$ & $30 \mathrm{GeV}$ & $40 \mathrm{GeV}$ & $45 \mathrm{GeV}$ & \\
\hline 0$)^{*}$ & 6443 & 64184 & 10852 & 7401 & $|Z|<50 \mathrm{~cm},-10<t<25 \mathrm{~ns}$ \\
\hline \multirow[t]{2}{*}{ 1) } & 1636 & 18327 & 3134 & 2198 & $|\eta|<0.7$ \\
\hline & $25.4 \%$ & $28.6 \%$ & $28.9 \%$ & $29.7 \%$ & \\
\hline \multirow[t]{2}{*}{ 2) } & 1550 & 18016 & 3086 & 2180 & P) $10 \mathrm{GeV}, 0.05<\mathrm{EMF}<0.95$ \\
\hline & $94.7 \%$ & $98.3 \%$ & $98.5 \%$ & $99.2 \%$ & \\
\hline \multirow[t]{2}{*}{ 3) } & 255 & 6397 & 1161 & 805 & $\Delta \phi>100^{\circ}, M_{J J}>60,65,80,90 \mathrm{Gel}$ \\
\hline & $16.5 \%$ & $35.5 \%$ & $37.6 \%$ & $36.9 \%$ & \\
\hline
\end{tabular}

* Preliminary event selection:

a) at least 1 central jet with Pt $>20 \mathrm{GeV}$, where central jet $\equiv$ jet with $|\eta|<1$ or physically in the central calorimeters CEM, CHA and WHA;

or b) at least 2 central jets and their total uncorrected energy > $33 \mathrm{GeV}$. 
Table 12 Unsmearing factors for the jet Et spectrum.

\begin{tabular}{|c|c|c|c|}
\hline \multirow{2}{*}{ Et $(\mathrm{GeV})$} & \multicolumn{3}{|c|}{$\sigma(\mathrm{E}) / 0.622 \mathrm{E}^{0.68}$} \\
\hline & $80 \%$ & $100 \%$ & $120 \%$ \\
\hline 32.5 & 0.848588 & 0.778755 & 0.704126 \\
\hline 37.5 & 0.843803 & 0.772149 & 0.696292 \\
\hline 42.5 & 0.841241 & 0.768602 & 0.692179 \\
\hline 47.5 & 0.840162 & 0.767090 & 0.690534 \\
\hline 52.5 & 0.840086 & 0.766959 & 0.690559 \\
\hline 57.5 & 0.840698 & 0.767776 & 0.691730 \\
\hline 62.5 & 0.841782 & 0.769247 & 0.693691 \\
\hline 67.5 & 0.843190 & 0.771170 & 0.696197 \\
\hline 72.5 & 0.844817 & 0.773403 & 0.699077 \\
\hline 77.5 & 0.846591 & 0.775843 & 0.702207 \\
\hline 82.5 & 0.848457 & 0.778417 & 0.705500 \\
\hline 87.5 & 0.850378 & 0.781072 & 0.708890 \\
\hline 92.5 & 0.852324 & 0.783768 & 0.712331 \\
\hline 97.5 & 0.854276 & 0.786478 & 0.715788 \\
\hline 105.5 & 0.857040 & 0.790325 & 0.720696 \\
\hline 115.5 & 0.860842 & 0.795632 & 0.727471 \\
\hline 125.5 & 0.864505 & 0.800760 & 0.734026 \\
\hline 135.5 & 0.868005 & 0.805676 & 0.740318 \\
\hline 145.5 & 0.871337 & 0.810368 & 0.746334 \\
\hline 157.5 & 0.875129 & 0.815723 & 0.753214 \\
\hline 172.5 & 0.879532 & 0.821960 & 0.761242 \\
\hline 189.5 & 0.884107 & 0.828461 & 0.769633 \\
\hline 214.5 & 0.889855 & 0.836661 & 0.780243 \\
\hline 244.5 & 0.896256 & 0.845804 & 0.791997 \\
\hline \multicolumn{4}{|c|}{ fit function $(E t+\alpha)^{-\beta}$} \\
\hline$a$ & 14.3 & 13.8 & 13.0 \\
\hline$\beta$ & 6.94 & 6.89 & 6.80 \\
\hline
\end{tabular}


Table 13 Unsmearing factors for the dijet mass spectrum.

\begin{tabular}{|c|c|c|c|}
\hline \multirow{2}{*}{$M_{J J}(G e V)$} & \multicolumn{3}{|c|}{$\sigma(M) / 0.386 \mathrm{E}^{0.77}$} \\
\hline & $80 \%$ & $100 \%$ & $120 \%$ \\
\hline 62.5 & 0.902046 & 0.855155 & 0.803016 \\
\hline 67.5 & 0.904567 & 0.858698 & 0.807564 \\
\hline 72.5 & 0.906809 & 0.861853 & 0.811620 \\
\hline 77.5 & 0.908817 & 0.864682 & 0.815261 \\
\hline 82.5 & 0.910626 & 0.867233 & 0.818548 \\
\hline 87.5 & 0.912264 & 0.869544 & 0.821529 \\
\hline 92.5 & 0.913753 & 0.871647 & 0.824244 \\
\hline 97.5 & 0.915114 & 0.873569 & 0.826726 \\
\hline 105.5 & 0.916865 & 0.876042 & 0.829922 \\
\hline 115.5 & 0.918999 & 0.879058 & 0.833819 \\
\hline 125.5 & 0.920825 & 0.881636 & 0.837150 \\
\hline 135.5 & 0.922399 & 0.883857 & 0.840019 \\
\hline 145.5 & 0.923766 & 0.885784 & 0.842505 \\
\hline 157.5 & 0.925188 & 0.887785 & 0.845082 \\
\hline 172.5 & 0.926671 & 0.889865 & 0.847753 \\
\hline 189.5 & 0.928043 & 0.891781 & 0.850200 \\
\hline 209.5 & 0.929328 & 0.893561 & 0.852459 \\
\hline 230.5 & 0.930328 & 0.894933 & 0.854180 \\
\hline 254.5 & 0.931229 & 0.896147 & 0.855674 \\
\hline 284.5 & 0.932014 & 0.897172 & 0.856890 \\
\hline 323.5 & 0.932563 & 0.897826 & 0.857577 \\
\hline 373.5 & 0.932786 & 0.897962 & 0.857512 \\
\hline 423.5 & 0.932568 & 0.897444 & 0.856504 \\
\hline 494.5 & 0.931456 & 0.894712 & 0.851360 \\
\hline \multicolumn{4}{|c|}{ fit function $M^{-a} e^{-\rho M}$} \\
\hline$a$ & 4.76 & 4.73 & 4.71 \\
\hline$\theta$ & 0.0032 & 0.0033 & 0.0034 \\
\hline
\end{tabular}


Table 14 Inclusive Jet Et Spectrum.

\begin{tabular}{|c|c|c|c|}
\hline $\begin{array}{l}\text { Et } \\
(\mathrm{GeV})\end{array}$ & $\begin{array}{l}\mathrm{d} \sigma / \mathrm{dEt} / \Delta \eta \\
(\mathrm{nb} / \mathrm{GeV})\end{array}$ & $\begin{array}{l}\text { sys. } \\
\text { error }\end{array}$ & $\begin{array}{l}\text { stat. } \\
\text { error }\end{array}$ \\
\hline 32.5 & 304.3 & 108.7 & 10.1 \\
\hline 37.5 & 148.2 & 52.9 & 7.0 \\
\hline 42.5 & 78.0 & 27.9 & 5.1 \\
\hline 47.5 & 43.0 & 15.4 & 0.65 \\
\hline 52.5 & 25.7 & 9.2 & 0.50 \\
\hline 57.5 & 14.8 & 5.3 & 0.38 \\
\hline 62.5 & 9.29 & 3.32 & 0.25 \\
\hline 67.5 & 6.04 & 2.16 & 0.17 \\
\hline 72.5 & 4.23 & 1.51 & 0.15 \\
\hline 77.5 & 2.84 & 1.02 & 0.12 \\
\hline 82.5 & 2.01 & 0.72 & 0.10 \\
\hline 87.5 & 1.62 & 0.58 & 0.090 \\
\hline 92.5 & 1.04 & 0.37 & 0.073 \\
\hline 97.5 & 0.625 & 0.223 & 0.056 \\
\hline 105.5 & 0.437 & 0.156 & 0.033 \\
\hline 115.5 & 0.234 & 0.084 & 0.025 \\
\hline 125.5 & 0.148 & 0.053 & 0.020 \\
\hline 135.5 & 0.102 & 0.036 & 0.016 \\
\hline \multirow[t]{2}{*}{145.5} & 0.0550 & 0.0196 & +0.0148 \\
\hline & & & -0.0096 \\
\hline \multirow[t]{2}{*}{157.5} & 0.0299 & 0.0107 & +0.0091 \\
\hline & & & -0.0056 \\
\hline \multirow[t]{2}{*}{172.5} & 0.0195 & 0.0070 & +0.0078 \\
\hline & & & -0.0043 \\
\hline \multirow[t]{2}{*}{189.5} & 0.0120 & 0.0043 & +0.0055 \\
\hline & & & -0.0028 \\
\hline \multirow[t]{2}{*}{214.5} & 0.00451 & 0.00161 & +0.00305 \\
\hline & & & -0.00124 \\
\hline \multirow[t]{2}{*}{244.5} & 0.00091 & 0.00033 & +0.00210 \\
\hline & : & & -0.00027 \\
\hline
\end{tabular}


Table 15 Inclusive Jet Pt Spectrum.

\begin{tabular}{|c|c|c|c|}
\hline $\begin{array}{l}\text { Pt } \\
(\mathrm{GeV})\end{array}$ & $\begin{array}{l}\mathrm{d} \sigma / \mathrm{dPt} / \Delta \eta \\
(\mathrm{nb} / \mathrm{GeV})\end{array}$ & $\begin{array}{l}\text { sys. } \\
\text { error }\end{array}$ & $\begin{array}{l}\text { stat. } \\
\text { error }\end{array}$ \\
\hline 32.5 & 216.1 & 76.7 & 8.6 \\
\hline 37.5 & 104.3 & 37.0 & 6.0 \\
\hline 42.5 & 53.5 & 19.0 & 0.74 \\
\hline 47.5 & 30.8 & 10.9 & 0.56 \\
\hline 52.5 & 17.8 & 6.3 & 0.43 \\
\hline 57.5 & 10.5 & 3.7 & 0.27 \\
\hline 62.5 & 6.72 & 2.38 & 0.19 \\
\hline 67.5 & 4.72 & 1.67 & 0.16 \\
\hline 72.5 & 2.84 & 1.01 & 0.12 \\
\hline 77.5 & 2.10 & 0.74 & 0.11 \\
\hline 82.5 & 1.75 & 0.62 & 0.096 \\
\hline 87.5 & 1.03 & 0.36 & 0.074 \\
\hline 92.5 & 0.753 & 0.267 & 0.063 \\
\hline 97.5 & 0.461 & 0.163 & 0.050 \\
\hline 105.5 & 0.312 & 0.111 & 0.029 \\
\hline 115.5 & 0.220 & 0.078 & 0.024 \\
\hline 125.5 & 0.106 & 0.038 & 0.017 \\
\hline \multirow[t]{2}{*}{135.5} & 0.0686 & 0.0243 & +0.0166 \\
\hline & & & -0.0112 \\
\hline \multirow[t]{2}{*}{145.5} & 0.0359 & 0.0127 & +0.0130 \\
\hline & & & -0.0074 \\
\hline \multirow[t]{2}{*}{157.5} & 0.0333 & 0.0118 & +0.0098 \\
\hline & & & -0.0061 \\
\hline \multirow[t]{2}{*}{172.5} & 0.0149 & 0.0053 & +0.0073 \\
\hline & & & -0.0036 \\
\hline \multirow[t]{2}{*}{189.5} & 0.00843 & 0.00299 & +0.00504 \\
\hline & & & -0.00222 \\
\hline \multirow[t]{2}{*}{214.5} & 0.00284 & 0.00101 & +0.00276 \\
\hline & & & -0.00086 \\
\hline \multirow[t]{2}{*}{244.5} & 0.00095 & 0.00034 & +0.00219 \\
\hline & & & -0.00028 \\
\hline
\end{tabular}


Table 16 Dijet Mass Spectrum.

\begin{tabular}{|c|c|c|c|}
\hline${ }_{(\mathrm{JJ}}^{\mathrm{GeV})}$ & $\begin{array}{l}\mathrm{d} \sigma / \mathrm{d} M_{\mathrm{JJ}} \\
(\mathrm{nb} / \mathrm{GeV})\end{array}$ & $\begin{array}{l}\text { sys. } \\
\text { error }\end{array}$ & $\begin{array}{l}\text { stat. } \\
\text { error }\end{array}$ \\
\hline 62.5 & 37.8 & 13.0 & 4.4 \\
\hline 67.5 & 25.5 & 8.8 & 0.62 \\
\hline 72.5 & 17.7 & 6.1 & 0.52 \\
\hline 77.5 & 13.2 & 4.5 & 0.45 \\
\hline 82.5 & 9.39 & 3.23 & 0.31 \\
\hline 87.5 & 6.46 & 2.22 & 0.26 \\
\hline 92.5 & 5.44 & 1.87 & 0.21 \\
\hline 97.5 & 4.17 & 1.43 & 0.18 \\
\hline 105.5 & 2.83 & 0.97 & 0.11 \\
\hline 115.5 & 1.72 & 0.59 & 0.083 \\
\hline 125.5 & 1.17 & 0.40 & 0.068 \\
\hline 135.5 & 0.756 & 0.260 & 0.055 \\
\hline 145.5 & 0.569 & 0.196 & 0.048 \\
\hline 157.5 & 0.321 & 0.110 & 0.029 \\
\hline 172.5 & 0.247 & 0.085 & 0.026 \\
\hline 189.5 & 0.121 & 0.042 & 0.016 \\
\hline 210.5 & 0.0708 & 0.0243 & 0.0120 \\
\hline \multirow[t]{2}{*}{230.5} & 0.0445 & 0.0153 & +0.0117 \\
\hline & & & -0.0076 \\
\hline \multirow[t]{2}{*}{254.5} & 0.0230 & 0.0079 & +0.0070 \\
\hline & & & -0.0043 \\
\hline \multirow[t]{2}{*}{284.5} & 0.0122 & 0.0042 & +0.0056 \\
\hline & & & -0.0029 \\
\hline \multirow[t]{2}{*}{323.5} & 0.00731 & 0.00251 & +0.00334 \\
\hline & & & -0.00171 \\
\hline \multirow[t]{2}{*}{373.5} & 0.00244 & 0.00084 & +0.00237 \\
\hline & & & -0.00074 \\
\hline \multirow[t]{2}{*}{423.5} & 0.00244 & 0.00084 & +0.00237 \\
\hline & & & -0.00074 \\
\hline \multirow[t]{2}{*}{494.5} & 0.000404 & 0.000139 & +0.000929 \\
\hline & & & -0.000118 \\
\hline
\end{tabular}


Fig. 63 The distributions of the interaction vertices ( $\mathrm{cm}$ in Z-direction) for all events from different trigger samples starting from run 7214 . The widths are found to decrease with increasing Et threshold. This might be due to the fact that the trigger hardware assumes $Z=0 \mathrm{~cm}$ and hence tends to under-calculate Et for events with vertices far from $Z=0 \mathrm{~cm}$. The mean and sigma when all distributions are combined are 3.60 and $36.65 \mathrm{~cm}$ respectively. 

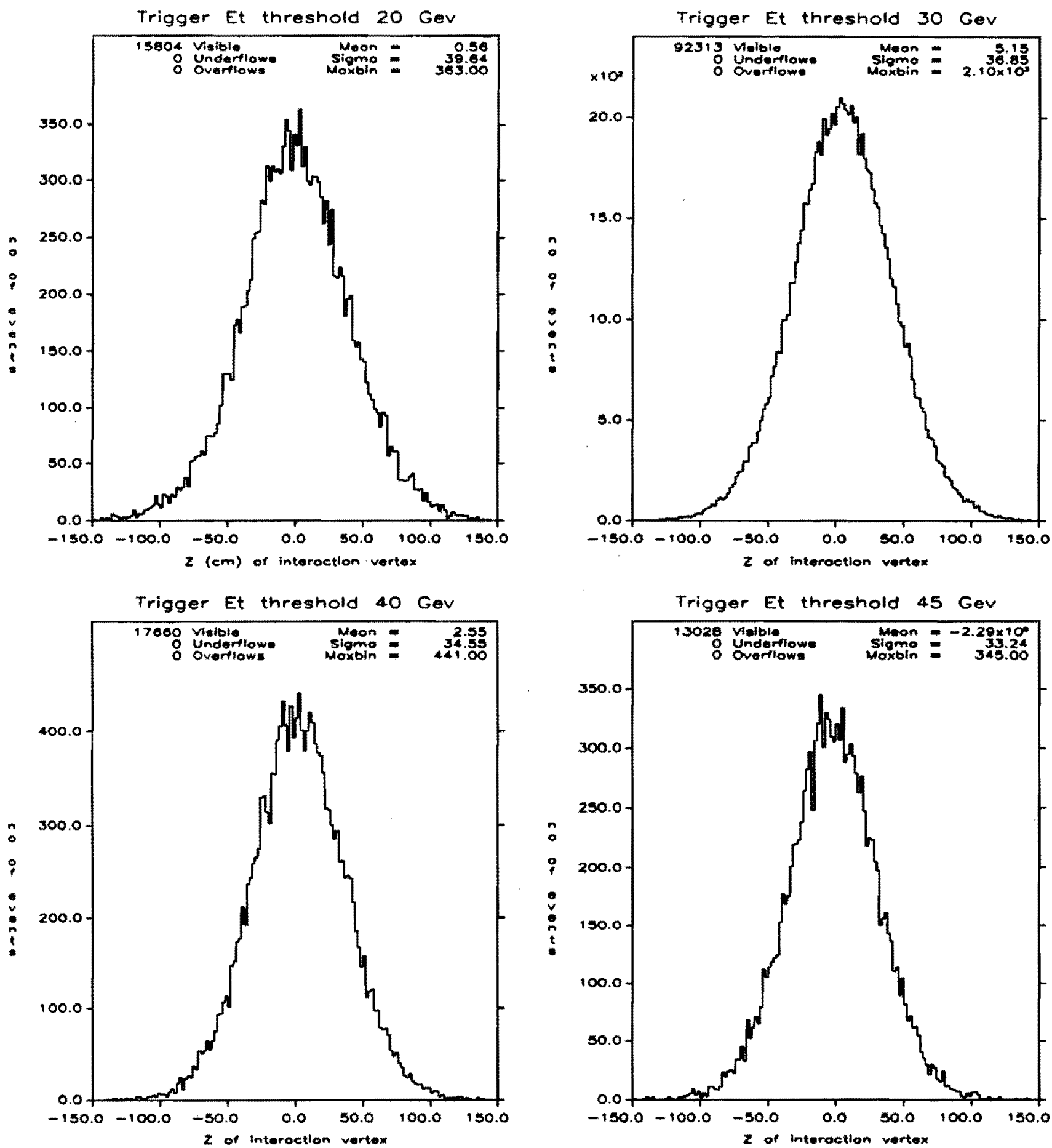


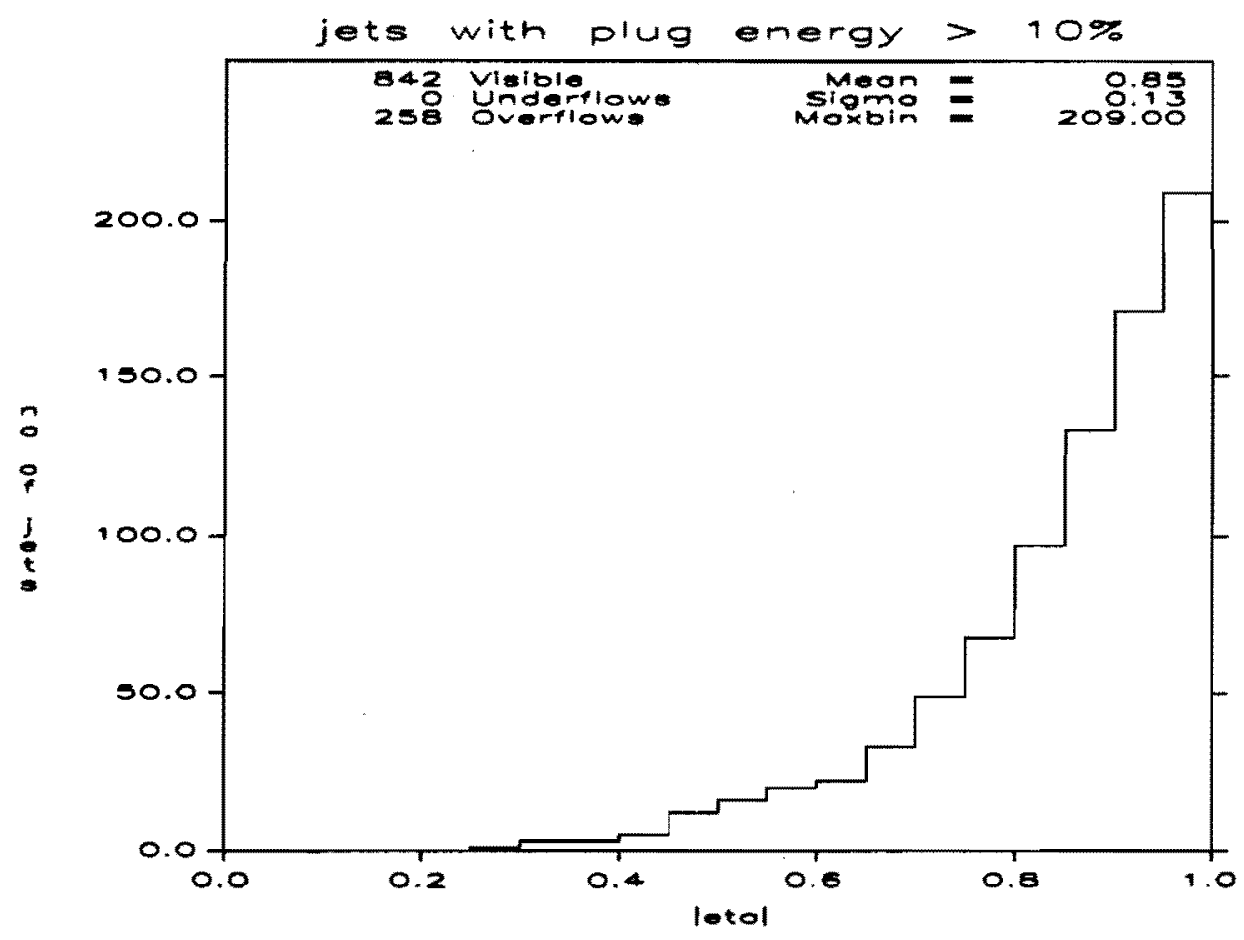

(a)

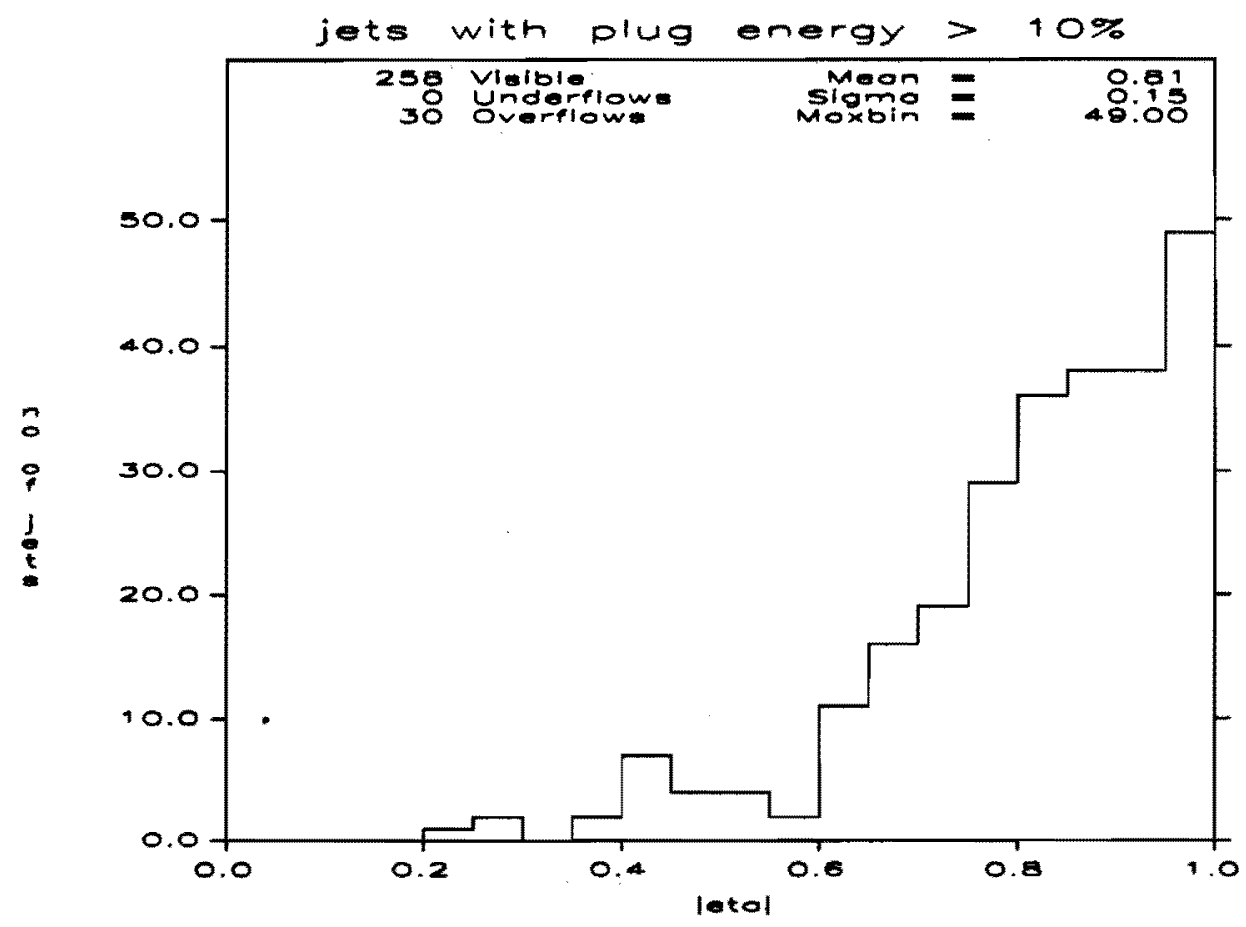

(b)

Fig. 64 The number of jets, as function of $\eta$, with more than $10 \%$ of energy coming from the Plug calorimeters. The event vertices are $30<|Z|<50 \mathrm{~cm}$. Plot (a) is cumulative from 0 to $\eta$, and (b) is for each $\Delta \eta=0.05$ bin. 


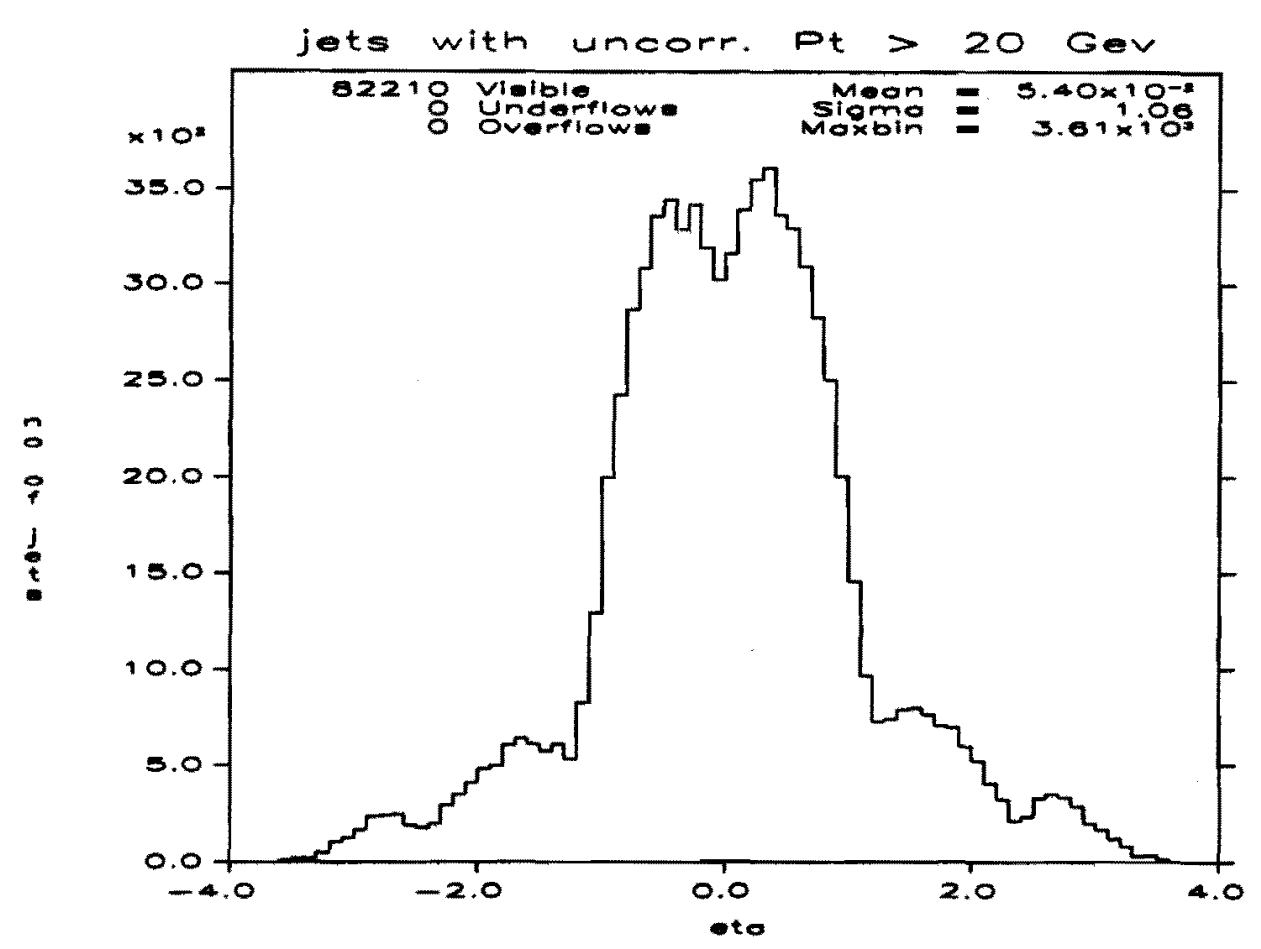

(a)

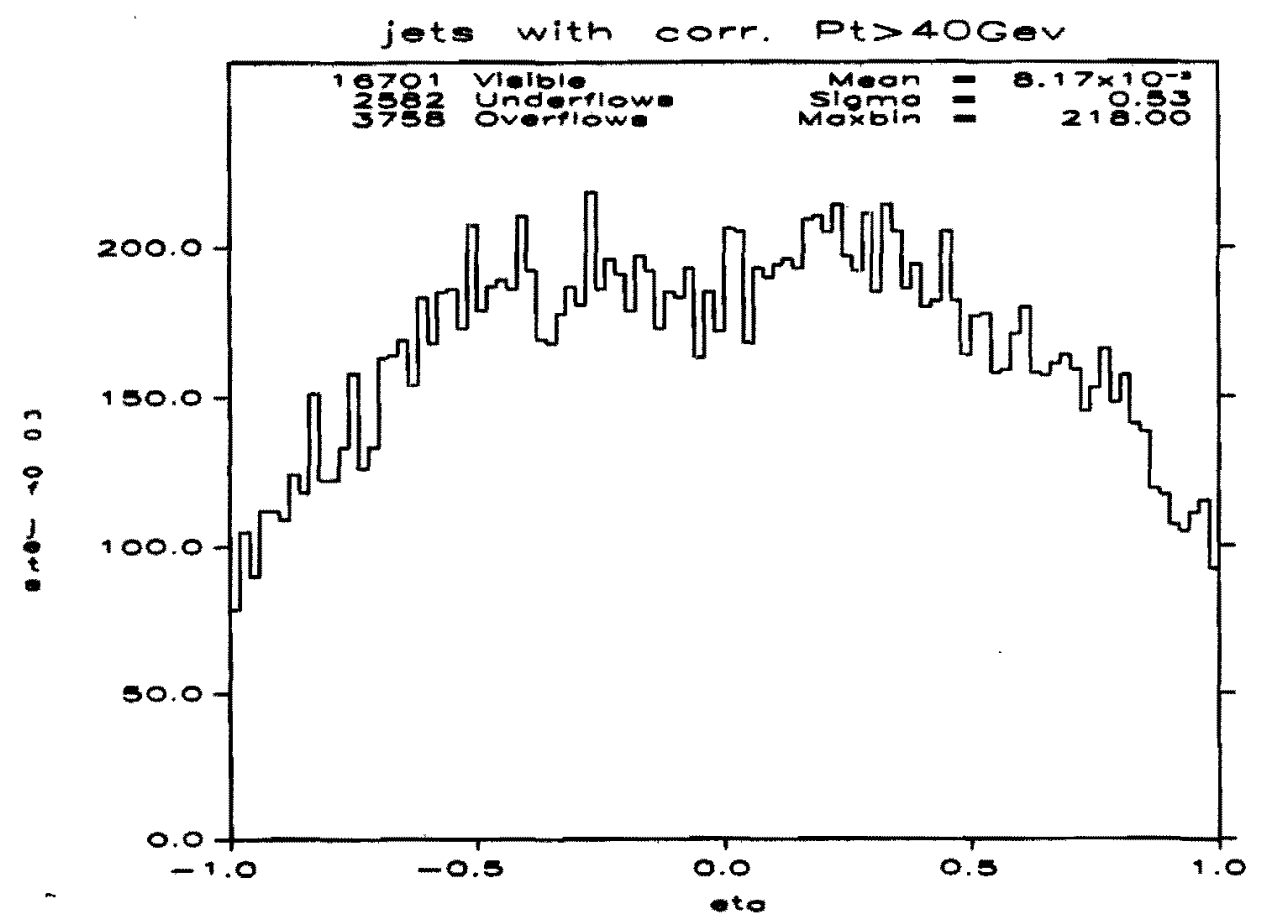

(b)

Fig. 65 The jet $\eta$ distributions: a) for jets with uncorrected $\mathrm{Pt}>20 \mathrm{GeV}$, and b) for jets with corrected $\mathrm{Pt}>40 \mathrm{GeV}$. 


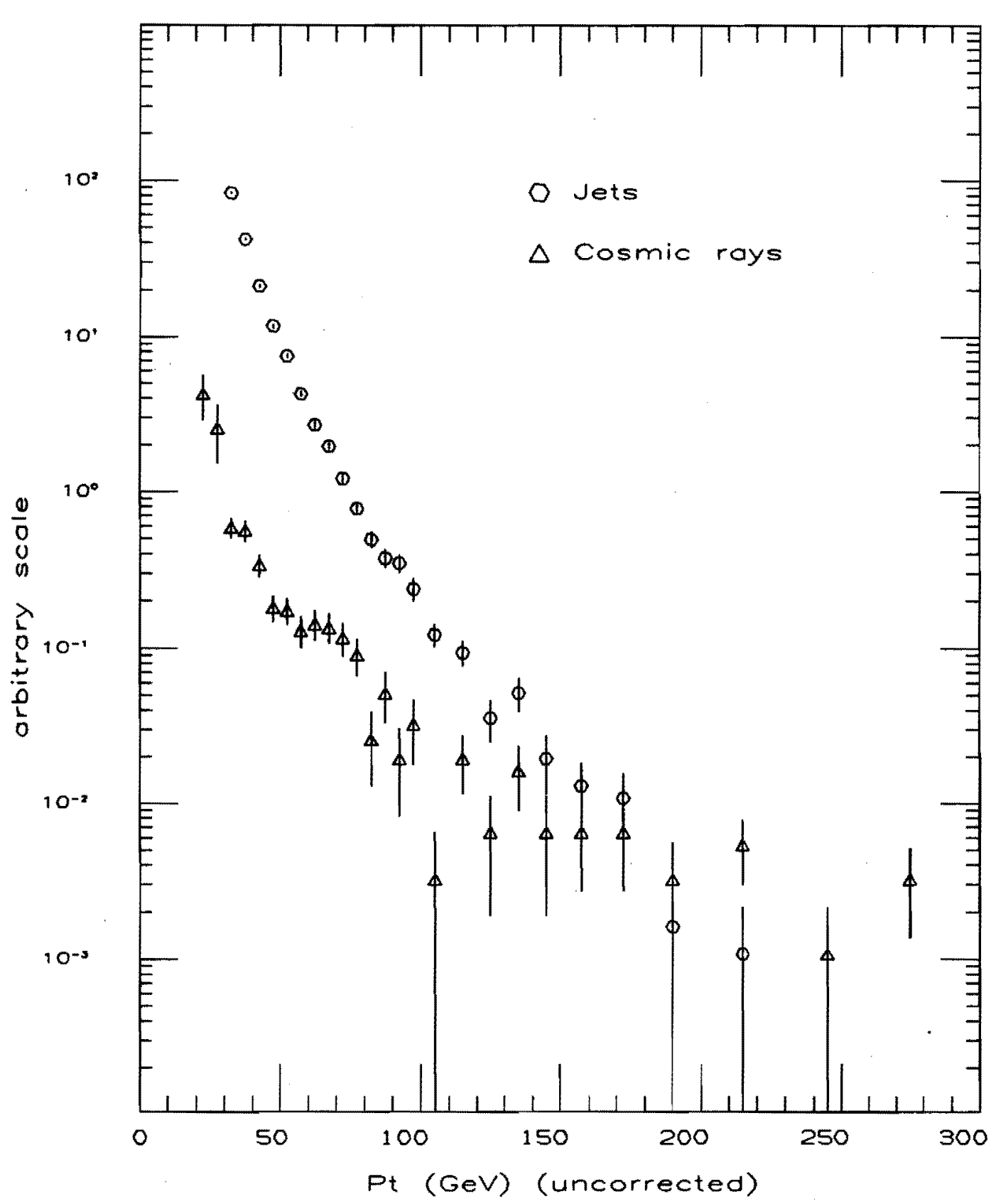

Fig. 66 Uncorrected Pt spectra for jets and cosmic rays in the central calorimeters $(|n| \leqq 0.7)$. 


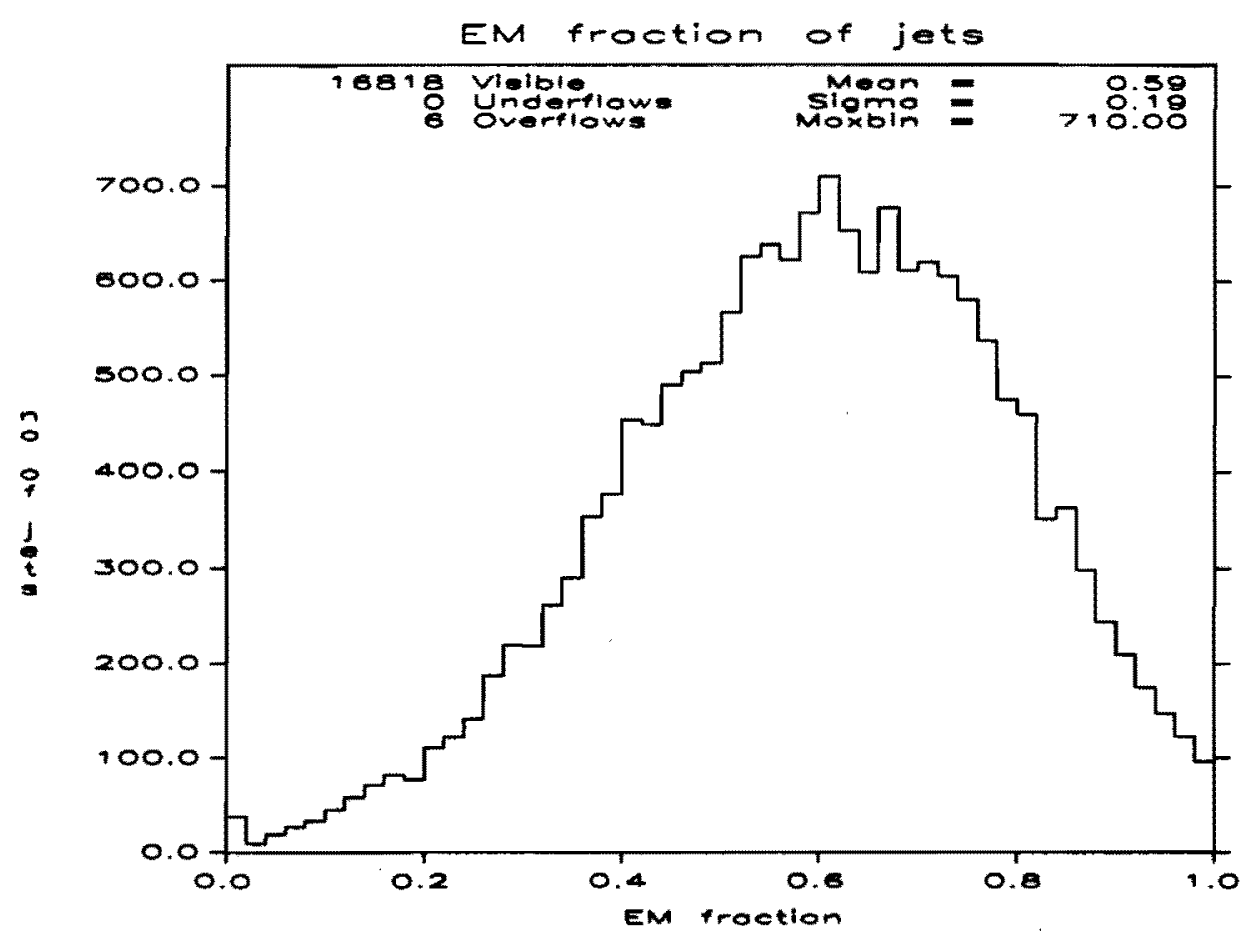

(a)

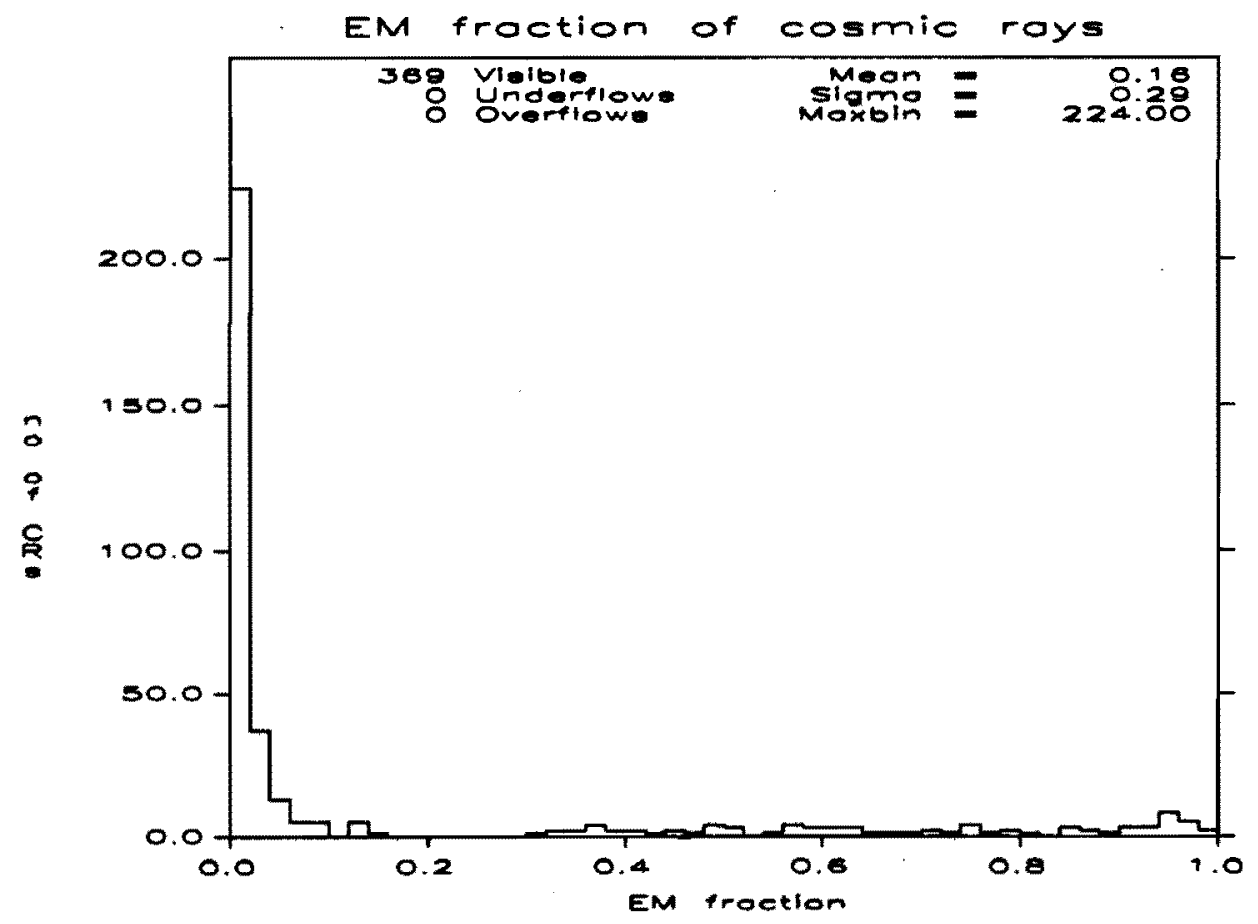

(b)

Fig. 67 The fraction of the cluster energy contained in the EM calorimeters (EMF) : a) for jets, and b) for cosmic rays. 


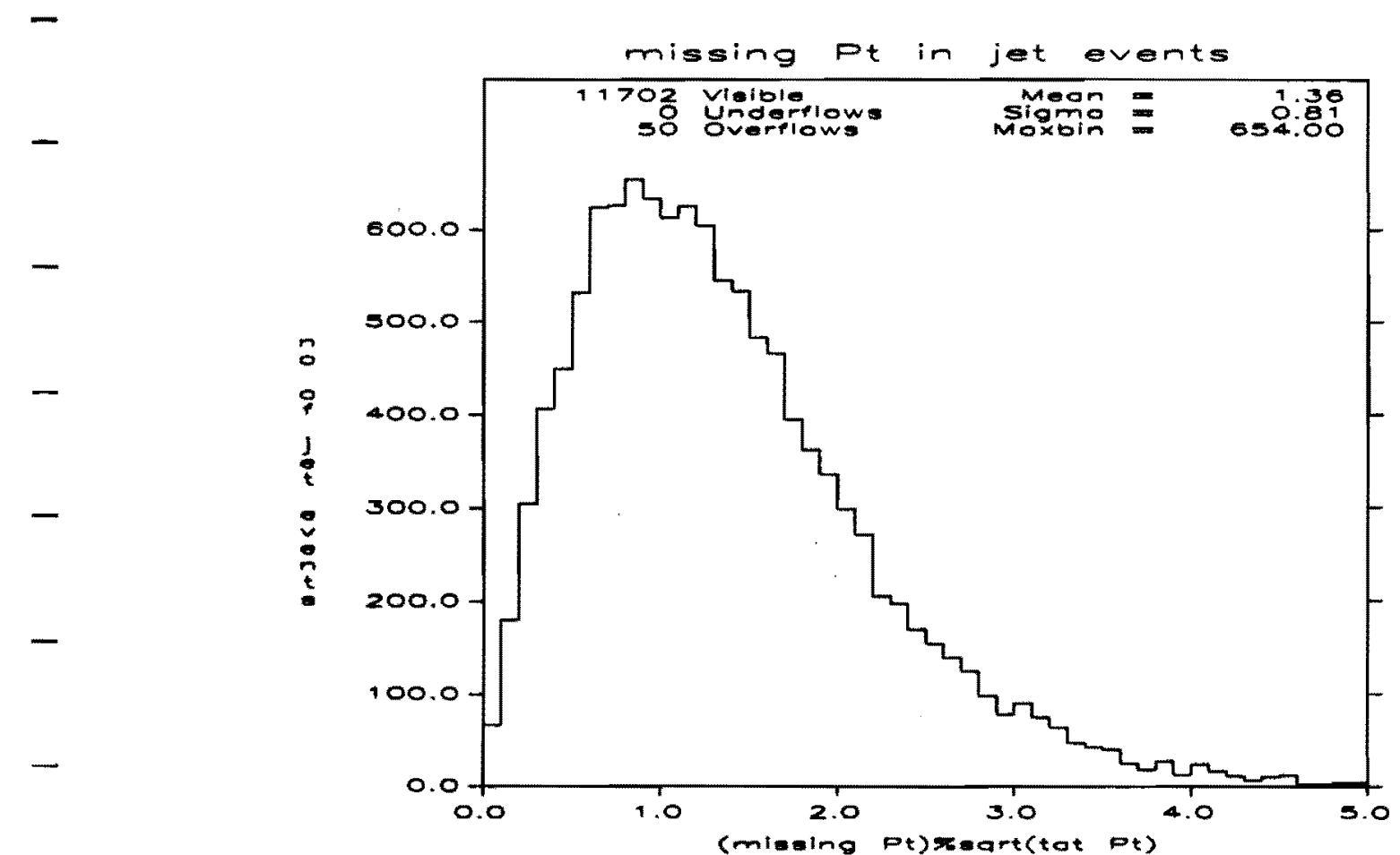

(a)

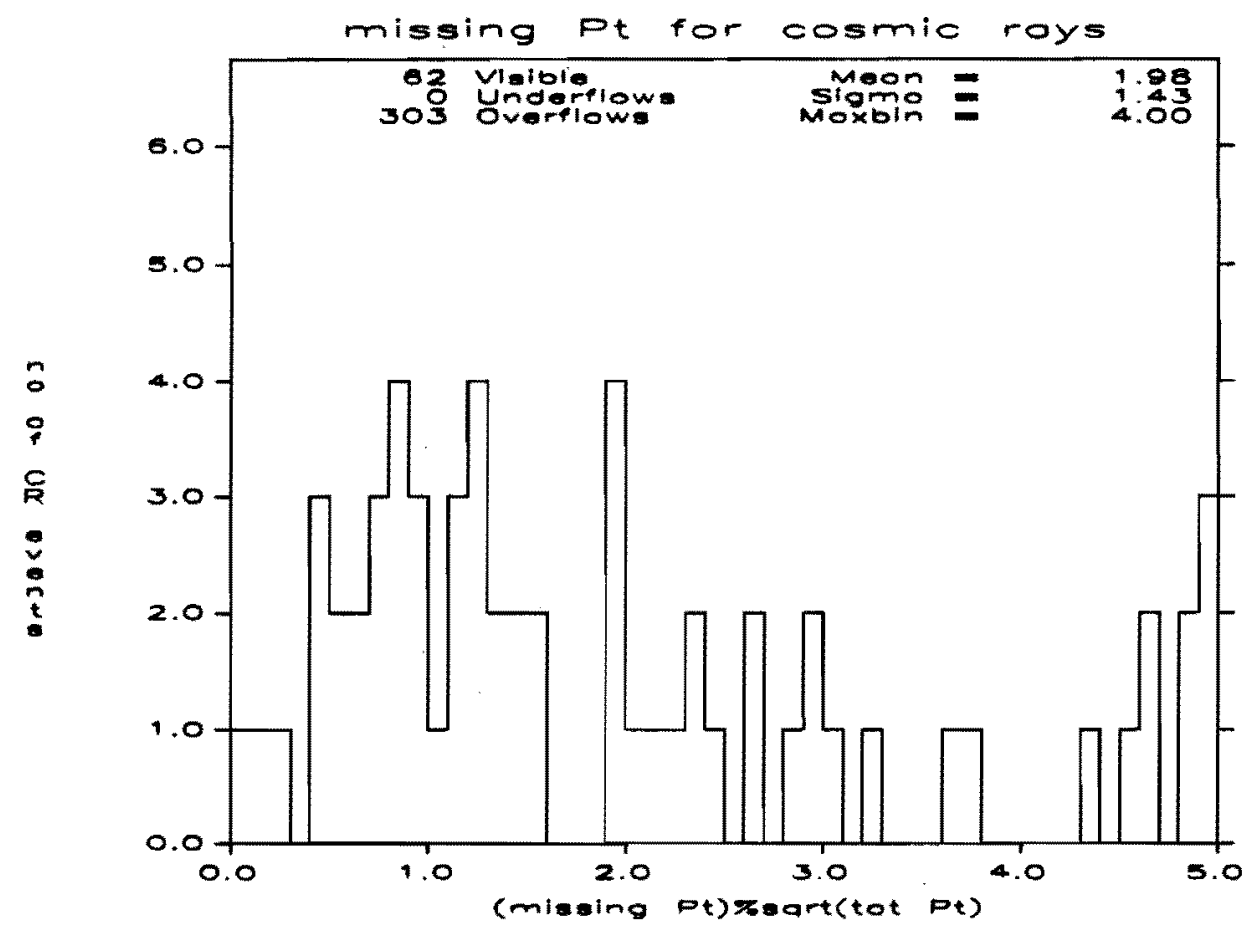

(b)

Fig. 68 The missing Pt significance ( $\equiv$ the ratio of the missing Pt and the square root of the scalar sum of all cluster Pt's): a) for jet events, and b) for cosmic ray events. 


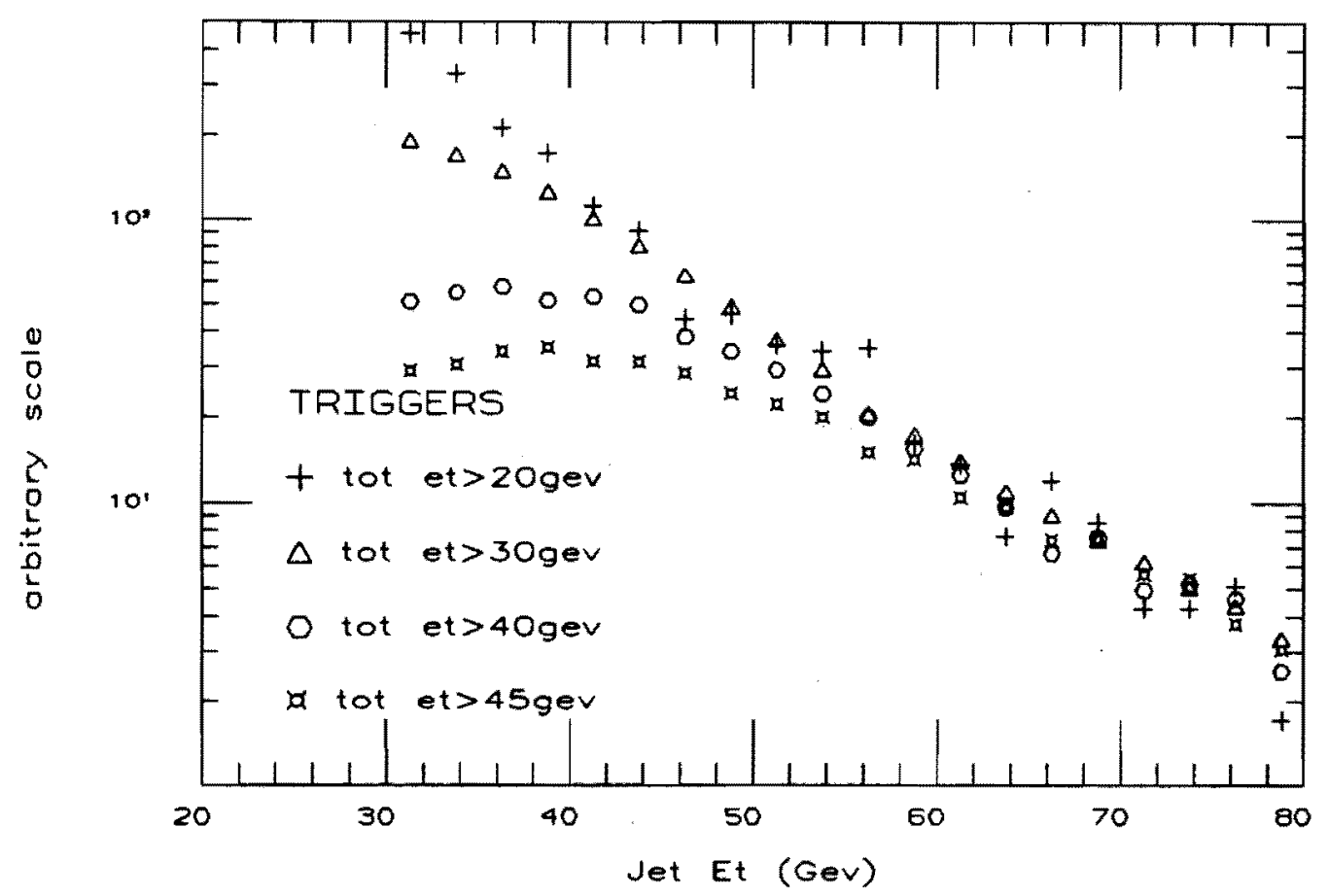

Fig. 69 The trigger effect on the jet Et spectrum. The stat. errors, which are large at large Et, are not shown.

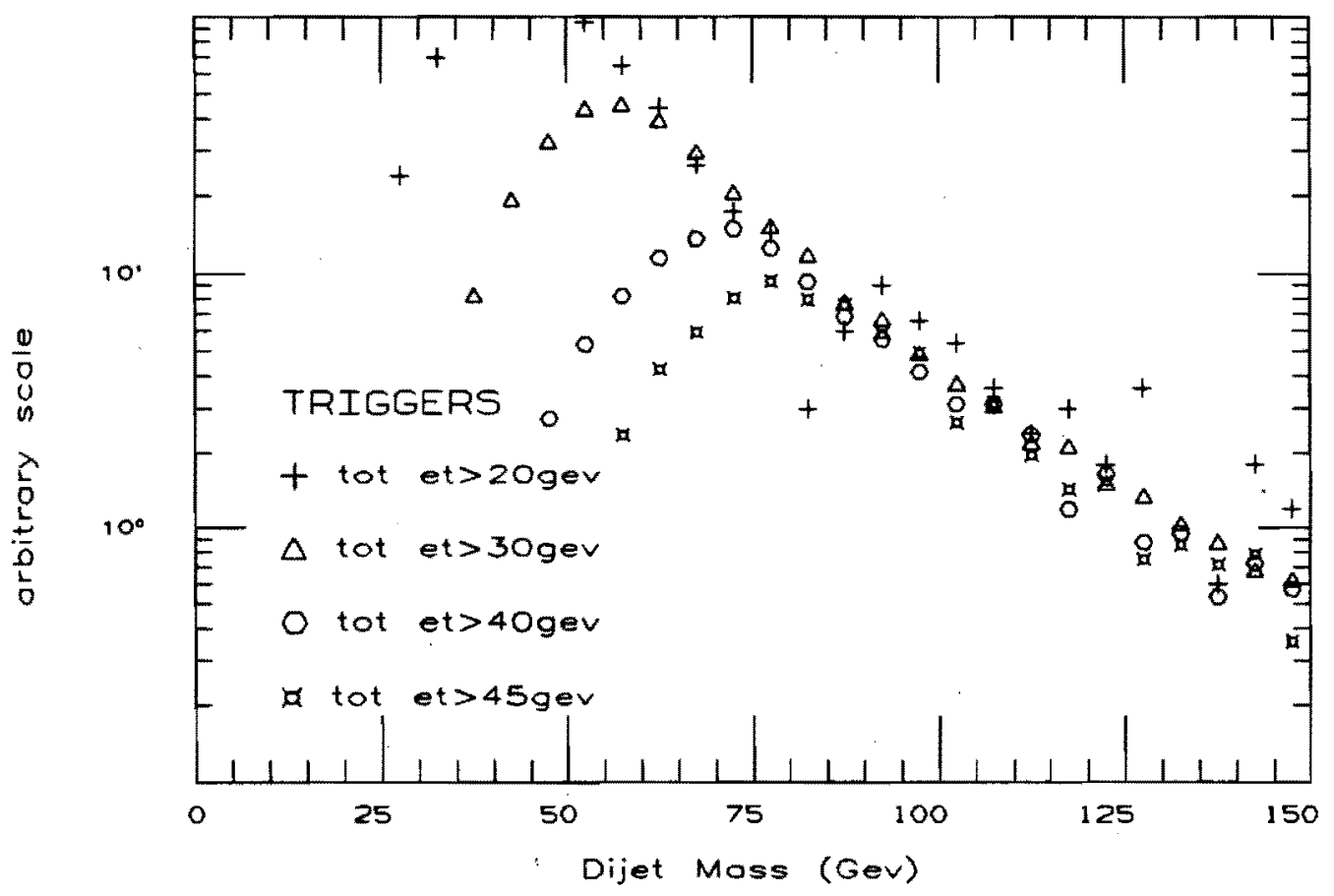

Fig. 70 The trigger effect on the dijet moss spectrum. The stat. errors, which are large for large mass, ore not shown. 


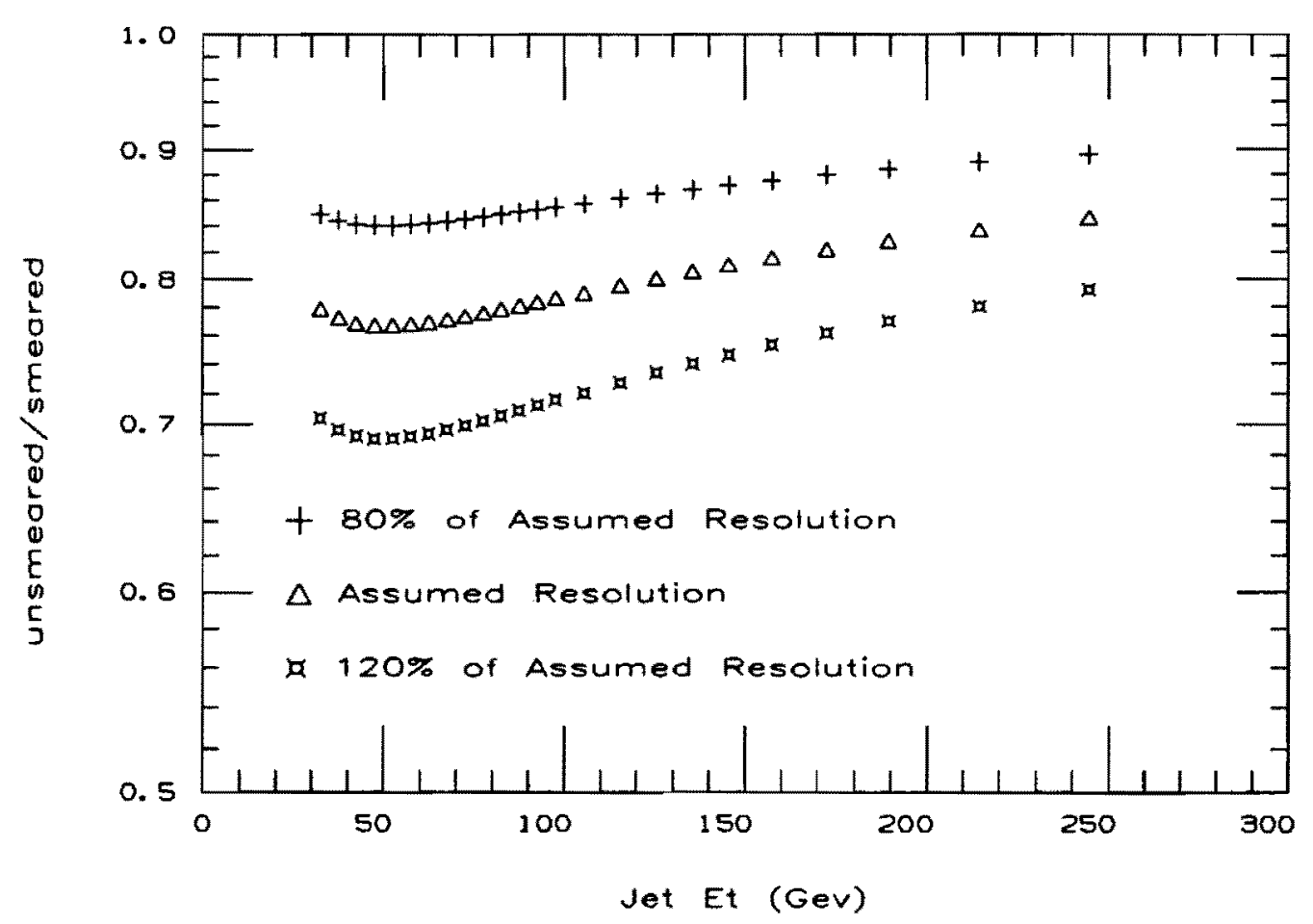

Fig. 71 Unsmearing Ratios for Jet Et Spectrum.

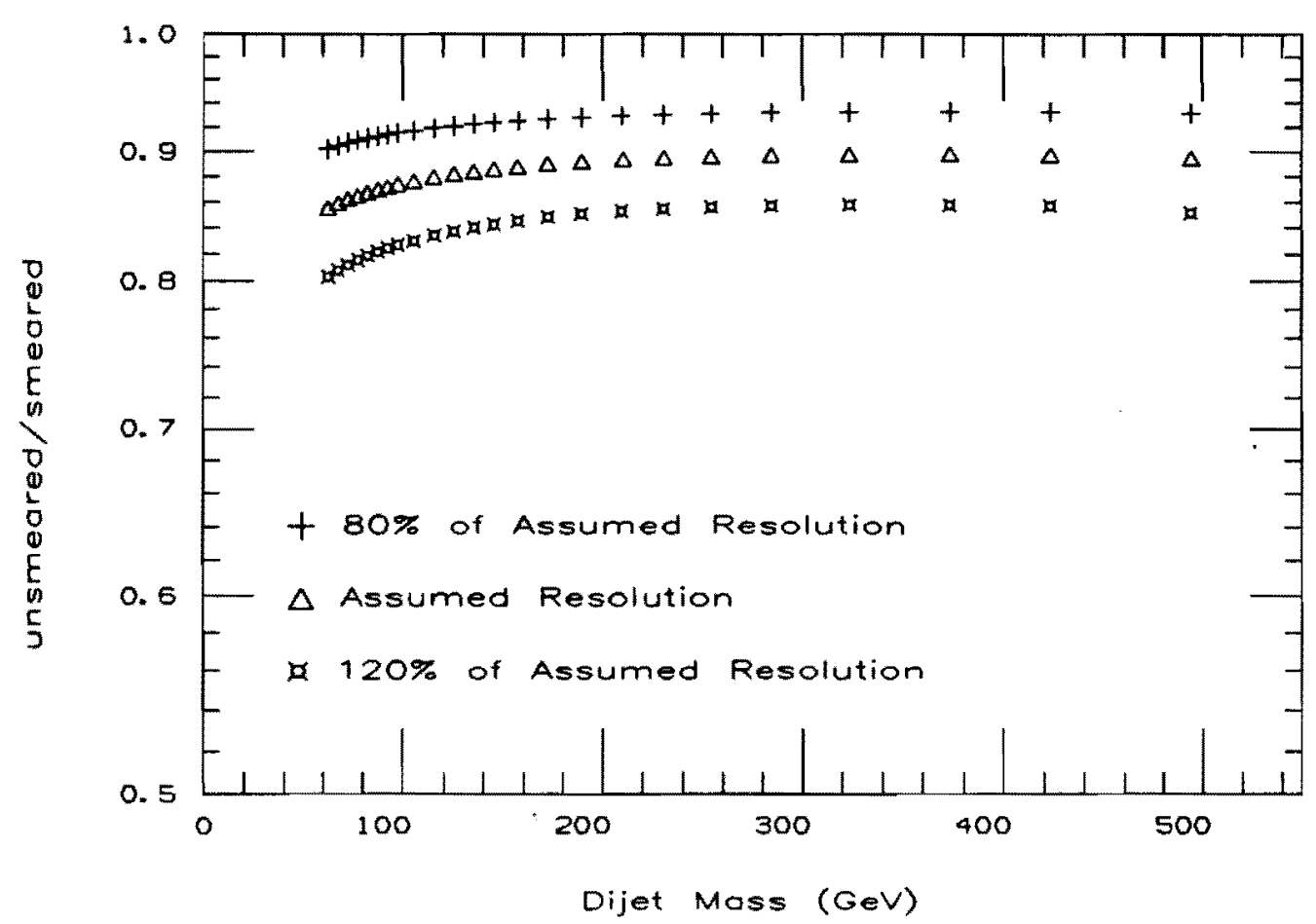

Fig. 72 Unsmearing Ratio for Dijet Mass Spectrum. 


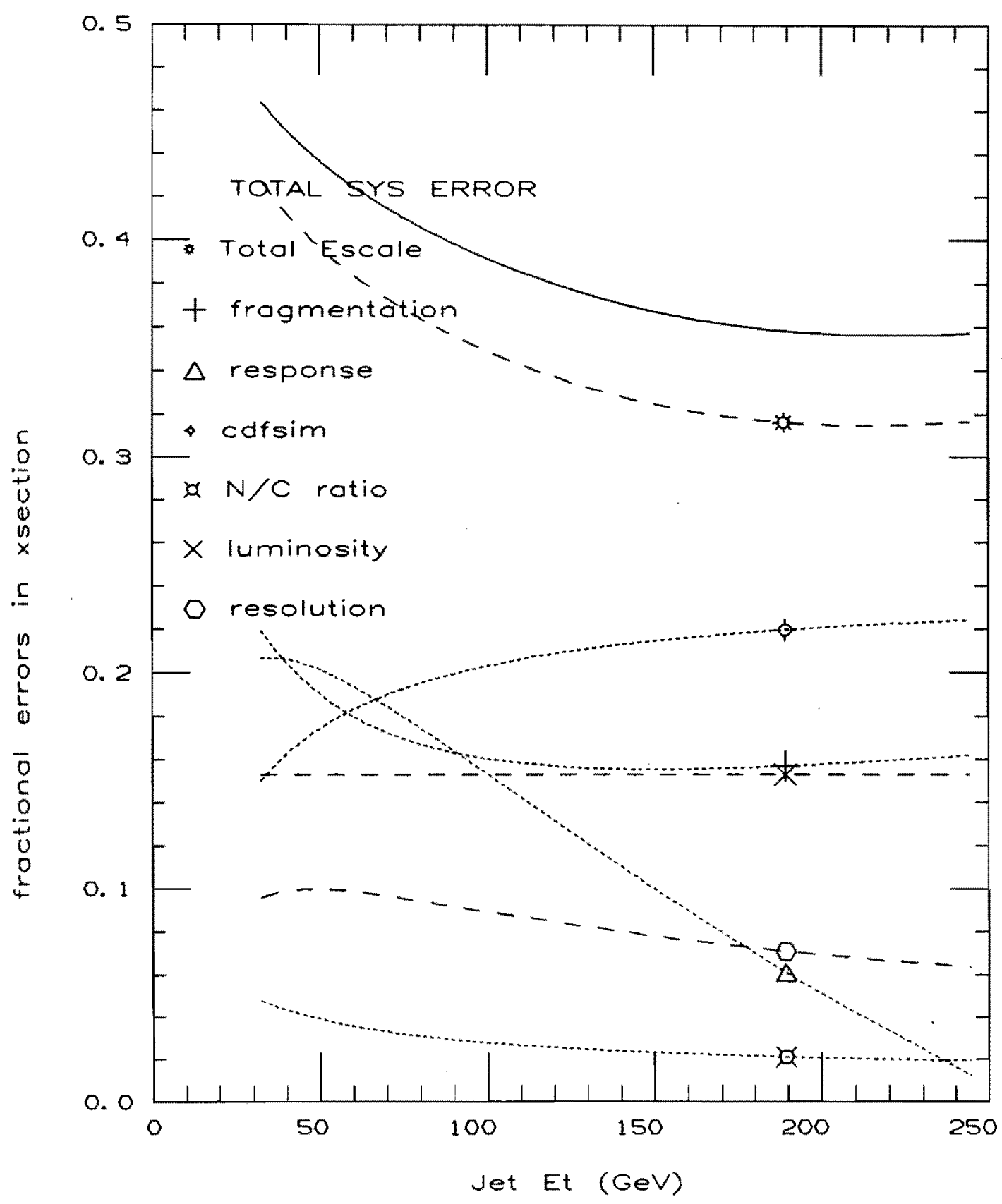

Fig. 73 Uncertainties in jet Et spectrum 


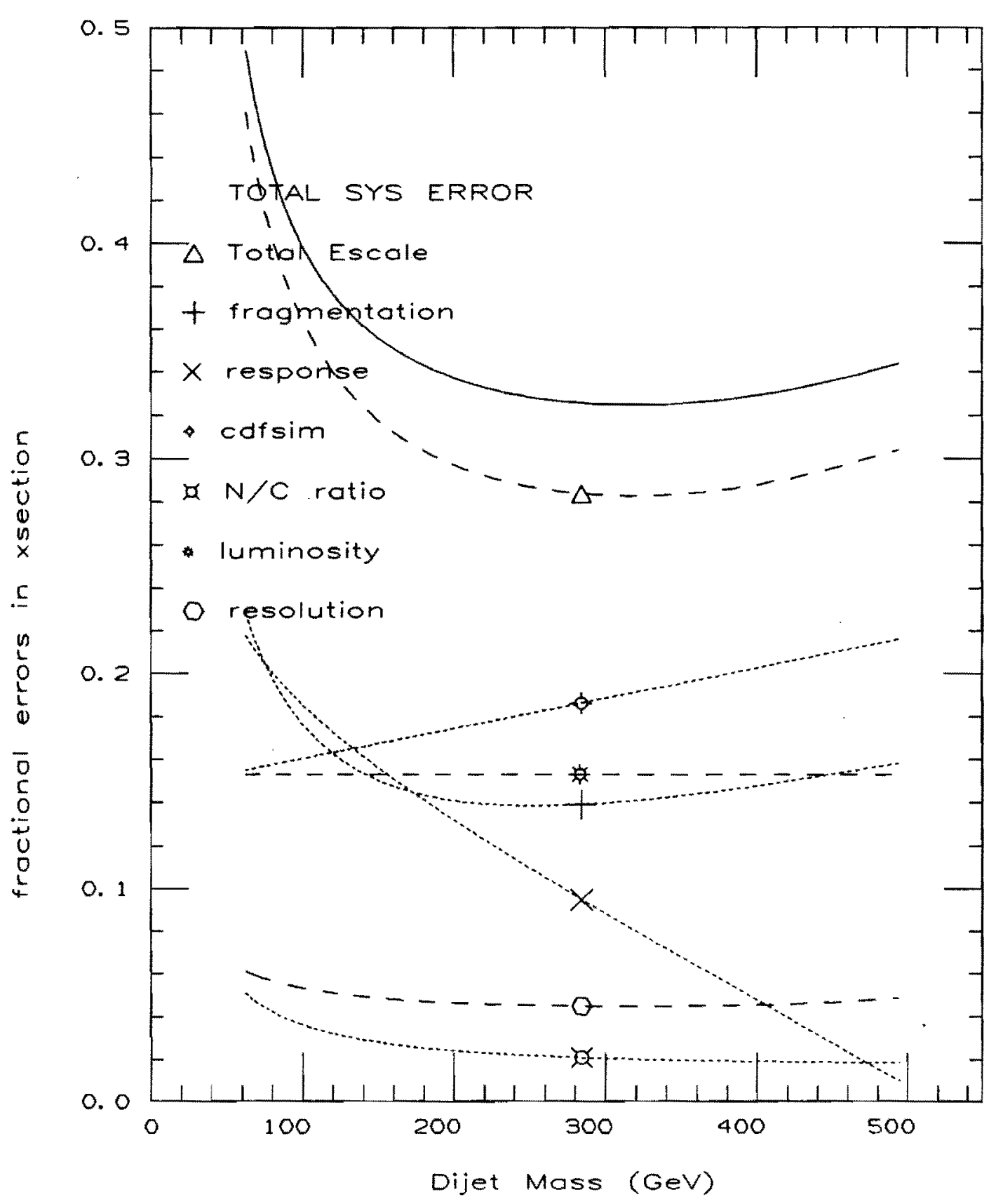

Fig. 74 Uncertainty in dijet mass spectrum 
CHAPTER 9

\section{PHYSICS ISSUES}

We will now compare our results with the lowest order $\left(\alpha^{2}\right)$ calculations for the 2 parton to 2 parton scattering. Besides the predictions of standard QCD, the consequences of compositeness of quarks, and also the possibility of chiral QCD will be considered. In the following all cross sections are calculated by programs provided by $E$. Eichten, and by J. Bagger, C. Schmidt and S. King. Their programs have been modified in one way or another according to our needs. For example our version of Eichten's program (modified by John Huth) employs the Monte Carlo integrator VEGAS, and the B-S-K program has been forced to use Eichten's $\alpha\left(Q^{2}\right)$ and structure function routines for consistency.

\subsection{Comparison with Standard QCD}

Four sets of structure functions will be compared: the two sets of DukeOwens [51] (D01, D02) and the two sets of Eichten-Hinchliffe-Lane-Quigg [12] (EHLQ1, EHLQ2). More details about these parameterizations can be found in Sect. A.3 and, of course, the original papers. We followed the EHLQ choice of scale parameters $M^{2}=Q^{2}=$ (const) $P P^{2}$, where $Q$ and $M$ are the momentum scales in the running coupling $\alpha\left(Q^{2}\right)$ and the structure functions $f\left(x, M^{2}\right)$. Their choice of smaller $Q(=P t / 2)$ is to minimize higher order corrections so that the Born term $\left(0\left(a^{2}\right)\right)$ can give a good approximation to the all-order results. It has been found that the $a^{3}$ corrections to the reactions $q_{i} q_{j} \longrightarrow q_{i} q_{j}$ are large and positive and are reduced by smaller choices of $\mathrm{M}^{2}$ and $Q^{2}$. But aside from this, there is no real argument to defend this choice of momentum scales. The $Q$ definition taken by EHLQ varies from $\mathrm{Pt} / 2$ to $\mathrm{Pt}$, which we will happily follow. Bigger Q (e.g. 2Pt) will give a lower cross section, but the higher order correction is bigger and will probably bring the cross section back to a level similar to that for a smaller Q. With the choice we have a total of 8 sets of calculations: 
(4 structure functions) $\cdot(2$-scales) as shown in Figs. 75 - 76. The fact that the cross section falls with higher $Q^{2}$ is due to smaller value of $a\left(Q^{2}\right)$ and shifting of the $f\left(x, Q^{2}\right)$ distribution toward lower $x$. The difference in cross sections is about $20 \%$ due to different choices of structure function, and about $40 \%$ due to different choices of Q-scale. The envelopes of these theoretical spectra are compared with the measured spectra in Figs. 77 - 79, where the theoretical envelopes are represented by two solid lines and the systematic errors by the dotted lines. The theoretical envelope and experimental bounds overlap nicely and we are not able to determine the best choice of structure function or the better choice of Q-scale. The shapes for different structure functions can be compared when we normalize theories to data by minimizing $\chi^{2}$ 's. This normalization is legitimate because our measurements have about $\pm 40 \%$ error due to the uncertainty in jet energy scale and luminosity determination. The normalized spectra are shown in Figs. $80-81$, and the minimized $\chi^{2}$ 's (not including the systematic errors) are listed in Tables $17-18$. Although the D02 parameterization seems to have better fit in the case of dijet mass, the large systematic uncertainty does not allow us to conclude that it is a better choice. In fact it has been pointed out by EHLQ that DO2 can not be trusted for very high $Q^{2}$ [52].

\subsection{The Possibility of Chiral QCD}

If the standard QCD is consistent with data, how are other non-standard models? An interesting hypothesis made by Frampton and Glashow [15] (see Sect. 1.7.2) is that the strong interaction might be chiral $\left(\mathrm{SU}(3)_{L} \times S U(3)_{R}\right)$ at high energy, but breaks at a certain energy scale down to the diagonal $\mathrm{SU}(3)_{V}$ we see today. Although the chiral group is uniquely assumed, there can be many different fermion representations of the group. But an octet of massive vector bosons, called axigluons by the authors, is universally required. As discussed in Sect. 1.7.2, the only axigluon masses not experimentally excluded seem to be in the regions $30<M_{A}<125 \mathrm{GeV}$ and $M_{A}$ $>310 \mathrm{GeV}$. But unless something unusual, the simplest models don't suggest $M_{A}>300 \mathrm{GeV}$. 
The jet production cross sections involving axigluons have been calculated by J. Bagger, C. Schmidt and S. King [17]. These authors excluded the region $125<M_{A}<275 \mathrm{GeV}$ using UA1 data (jet Pt spectrum). In their calculations the width of the axigluon is assumed to be

$$
\Gamma=N a_{A} M_{A} / 6,
$$

where $N$ is the number of decay channels (i.e. the number of light quarks), $a_{A}$ is the axigluon coupling, taken to be equal to the QCD coupling $a_{S}$ (for parity conservation), and $M_{A}$ is the axigluon mass. The value of $N$ can vary from 5 for the known quarks up to 18 for one of the models (the most promising one, but not all quarks are light) described by Frampton and Glashow. We have used the program of B-S-K to obtain dijet mass spectra with axigluon masses ranging from 200 to $400 \mathrm{GeV}$ and $N=5,10$ and 20 . The choice of $Q=P t$ and EHLQ2 again gives the steepest spectra, thus allowing us the most conservative limit on a possible axigluon.

To make a comparison with data, we have smeared the calculated spectra with the known detector resolution (Sect. 7.4.3). The reason for not comparing "unsmeared" spectra is the following. If we were to unsmear the measured spectrum by the procedure described in Sect. 8.4.1, a certain function must be assumed. But different functions, especially one with a bump and one without a bump, give rather different unsmearing factors, and we do not know a priori the true shape underlying the data. The difference between the two approaches can be clearly seen by comparing Fig. 82 and Fig. 84, where the data are compared in different ways to the axigluon calculations for $\mathbf{N}=10$.

The procedure of smearing a theoretical spectrum is in principle similar to that we described in Sect. 8.4.1, but here we do not have any analytic function for the spectrum and a different technique is required. We calculated differential cross sections for dijet masses from $10 \mathrm{GeV}$ up to $705 \mathrm{GeV}$ at intervals of $5 \mathrm{GeV}$. The lowest and highest masses have been taken well beyond the range of the measured spectrum because these outer regions will affect the data region through resolution smearing. We then extrapolate the calculated values to every $1 \mathrm{GeV}$ by fitting 3 consecutive points (e.g. 45,50 and 55) on a logarithmic plot with a quadratic function, which then gives the values for the points between the first and second 
points (i.e. $46,47,48$ and 49 ). Finally we smear the spectrum by sending the content of each $1 \mathrm{GeV}$ bin into other bins via the assumed Gaussian resolution.

To look for a sign of a possible axigluon, we normalize each spectrum (convoluted with the detector resolution) calculated with one value of $M_{A}$ to the data by minimizing the $\chi^{2}$ for bins in the low mass region $\left(60<M_{J J}\right.$ ( $120 \mathrm{GeV}$ ), and calculate the $\chi^{2}$ for the bins under the axigluon bump (Figs. 83 - 85). The $\chi^{2}$ is calculated assuming Poisson statistics from the number of events in each bin and the theoretical number obtained by integrating the smeared spectra (actually by summing all $1 \mathrm{GeV}$ bins) over the range of each data bin multiplied by the integrated luminosity. The Poisson theory says if we found $r$ entries in the data, then the likelihood for the theoretical value to be $m$ is given by $P(m)=\exp (-m) m^{r} / r !$. The $X^{2}$ can be defined by recasting $P(m)$ in a Gaussian form: $P(m) \equiv\left[\exp (-r) r^{r} / r !\right]$ $\exp \left(-\chi^{2}(m)\right)$. (Recall that the $\chi^{2}$-distribution for $n$ degrees of freedom is derived through the integration of gaussian likelihood $\Pi \int \exp \left(-\chi^{2}{ }_{i}\right) \mathrm{d} \chi_{i}=$ $\left.\int \cdots \int \exp \left(-\Sigma \chi_{i}^{2}\right) d \chi_{1} \cdots d \chi_{n} \equiv \int \exp \left(-R^{2}\right) d R \int \cdots \int J d \theta_{1} \cdots d \theta_{n-1} \cdot\right)$

The effect of the uncertainty in the jet energy scale must also be considered. We allow both edges of each data bin to vary according to the overall uncertainty in the jet energy (Fig. 59). The mass value at each edge is multiplied by a factor $1+a f(M)$, where $f(M)$ is the fractional uncertainty in the mass scale as a function of mass and a varies from -1.0 to 1.0 at 0.1 steps. In this rescaling of bins, the number of entries in each bin remains the same, but the differential cross section and the statistical error in the cross section (and the bin center) must be scaled according to the new bin size. When the bins are varied, the $\chi^{2}$ for the axigluon bump region also changes, and we use the smallest (or greatest) $\chi^{2}$ to disprove (prove) the axigluon hypothesis. The bins to be compared, three or four bins under the axigluon bump, were chosen by eye. The data show no sign of a deviation from $Q C D$, and we have excluded (at $95 \%$ confidence level) the axigluon masses:

$$
\begin{aligned}
& N=5,120<M_{A}<210 \mathrm{GeV} \\
& N=10,120<M_{A}<150 \mathrm{GeV} \\
& N=20, \text { no limit }
\end{aligned}
$$


It seems we can not do better than the CERN groups in the lower mass region (UA1 was able to exclude the mass region from 110 to $310 \mathrm{GeV}$ for an axigluon width $\Gamma_{A}<0.4 M_{A}$, which is equivalent to $N \leq 24$ if $a_{s}$ is taken to be 0.1 ) because the contribution of the axigluon to a mass bump is due to $q q$ or q $\bar{q}$ scattering (see Sect. 1.7.2 and Fig. 2) and at the Tevatron energy the dominant process is gluon scattering. On the other hand, the statistics of our 1987 data do not allow us to exclude (or confirm) higher masses where the CDF should have the advantage over the Spps experiments due to a higher available energy. About 200 times more data have been collected in the 1988-1989 run.

The effect of the axigluon on the inclusive jet Et spectrum is shown in Fig. 86 .

\subsection{Compositeness of Quarks}

The multiplicity of quarks has lead one to speculate that they might be composite as well (see Sect. 1.7.1). As history repeats itself, the quarks and leptons might be composed of more "fundamental" particles, often called 'preons', which are bounded together by a new kind of asymptotically free and infrared confining gauge force [53]. Any substructure of quarks and leptons will modify the gauge field própagator by a form factor

$$
F\left(Q^{2}\right)=1+Q^{2} / \Lambda^{2} \text {, }
$$

where $\Lambda$ is the compositeness scale and $Q$ the four momentum carried by gauge field. However if $\Lambda$ is large compared to the available energy, the composite interaction can be approximated by the 4-fermion contact terms of the form in Eq. (1.9) which is the low energy manifestation of constituent exchanges.

Jet Pt spectra including the contact interaction have been calculated by Eichten's program with the compositeness scale $\Lambda$ varied from $400 \mathrm{GeV}$ to 700 $\mathrm{GeV}$ (Fig. 87). We again choose EHLQ2 and $Q=P t$ because they together give the steepest spectra and thus allow the most conservative limit on the compositeness scale $\Lambda$ (Fig. 88). We observe that for $\Lambda>600 \mathrm{GeV}$ the calculated spectra do not deviate much from the data. But the number of jets predicted for the vast $\mathrm{Pt}$ region above the highest bin (integrated 
from the high edge of the last bin to infinity) is quite different from that predicted by QCD [54]. The QCD prediction in this region is in good agreement with data ( 0 jet). How different the predictions are depends on where the spectrum with compositeness starts to bend up. Intuitively we expect that the limit on the compositeness scale one can set is the $\Lambda$ which makes the spectrum bend up somewhere around the highest data bin.

We have allowed the starting edge of the high Et bin (up to infinity) to vary and evaluated the $\chi^{2}$ and the probability of agreement with data for a particular $\Lambda(=700 \mathrm{GeV})$ and for the standard $Q C D(\Lambda=\infty)$, as shown in Fig. 90 and Fig 91 . Again we normalized the theory (convoluted with detector resolution, see Fig. 89) to the data by minimizing the $\chi^{2}$ for bins in the low Et region (from 60 to $165 \mathrm{GeV}$ ), and have calculated $\chi^{2}$ for the high Et bin from the number of jets observed and the number expected. Whenever the edge hits the Et of an observed jet, the $\chi^{2}$ drops. To set a I imit on $\Lambda$, we don't want to move the edge too low because the inclusion of the lower Et region before spectrum bending would obscure the significance of the difference (for example, the probability of observing 1 when expecting 4 is $9.5 \%$, but that of observing $1+3$ when expecting $4+3$ is $22.7 \%$ ). We found that choosing the second highest jet $E t$ as the starting edge gives a limit higher than any other choices would give (this reminds us of the Kolmogorov-Smirnov test where the greatest distance between two cumulative distributions is used to give the significance level of a disproof of the hypothesis that the two distributions are the same). But to be very conservative we would rather stay with the highest jet Et.

Because the numbers involved are small (<5), the use of a $\chi^{2}$-distribution to convert $\chi^{2}$ to probability is not fully justified. When there is only one bin (1 degree of freedom), it's rather simple to get the probability without going through the $\chi^{2}$-distribution: The likelihood for the theoretical value to be $m$ when actually observing $r$ is given by $P(m)=$ $\exp (-m) m^{r} / r !$, and the probability of agreement is just the area under the likelihood function with $P(\mu)<P(m)$. Table 19 lists the probabilities calculated by this method. We have varied the compositeness scale $\Lambda$ in 10 GeV steps until a $95 \%$ confidence level is reached. A limit $\Lambda>680 \mathrm{GeV}$ is set by taking the highest jet Et as the starting edge of the high Et bin 
(choosing the second highest Et gives $\Lambda>800 \mathrm{GeV}$ ). This is compared to the I imit $400(370)$ GeV set by UA1(UA2) $[55,56]$. 
Table 17 Comparing the QCD predicted Et spectra with data. QCD spectra are scaled to minimize $\chi^{2}$ 's (for 24 d.o.f).

\begin{tabular}{cllllll}
\hline & $Q \equiv$ & & D01 & D02 & EHLQ1 & EHLQ2 \\
\hline smeared & & & & & & \\
& & & & & & \\
& & $\chi^{2}$ & 22.07 & 35.55 & 25.11 & 28.25 \\
& & scale-up & 1.57 & 1.37 & 1.40 & 1.26 \\
& & $\chi^{2}$ & 36.44 & 22.36 & 24.90 & 24.59 \\
unsmeared & scale-up & 1.18 & 1.00 & 1.05 & 0.93 \\
& & & & & & \\
& & $\chi^{2}$ & 19.99 & 20.13 & 19.95 & 21.92 \\
& scale-up & 1.58 & 1.39 & 1.41 & 1.26 \\
& Pt/2 & $\chi^{2}$ & 35.85 & 19.03 & 24.87 & 25.04 \\
& scale-up & 1.15 & 0.99 & 1.03 & 0.91
\end{tabular}

* This can not be used to judge the goodness of any structure function as the systematic errors are not included.

Table 18 Comparing the QCD predicted $M_{J J}$ spectra with data. QCD spectra are scaled to minimize $\chi^{2}$ 's (for 24 d.o.f).

\begin{tabular}{|c|c|c|c|c|c|}
\hline$Q \equiv$ & & D01 & D02 & EHLQ1 & EHLQ2 \\
\hline \multicolumn{6}{|l|}{ smeared } \\
\hline Pt & $x^{2}$ & 39.33 & 17.64 & 29.24 & 30.30 \\
\hline & scale-up & 1.46 & 1.31 & 1.24 & 1.13 \\
\hline $\mathrm{Pt} / 2$ & $x^{2}$ & 91.94 & 39.51 & 66.61 & 73.33 \\
\hline & sca le-up & 1.14 & 1.00 & 0.98 & 0.88 \\
\hline \multicolumn{6}{|l|}{ unsmeared } \\
\hline Pt & $x^{2}$ & 30.20 & 15.24 & 23.35 & 24.43 \\
\hline & scale-up & 1.43 & 1.28 & 1.22 & 1.11 \\
\hline $\mathrm{Pt} / 2$ & $x^{2}$ & 69.13 & 29.86 & 49.92 & 55.17 \\
\hline & scale-up & 1.12 & 0.98 & 0.95 & 0.85 \\
\hline
\end{tabular}

* This can not be used to judge the goodness of any structure function as the systematic errors are not included. 
Table 19 Probability of agreement with data for various compositeness scales. A bin starting from the highest or the 2nd highest jet Et to $\infty$ is compared.

\begin{tabular}{|c|c|c|c|c|}
\hline $\begin{array}{l}\text { starting } \\
\text { edge (GeV) }\end{array}$ & $\begin{array}{l}\text { number } \\
\text { observed }\end{array}$ & ${ }^{\Lambda_{C}}(\mathrm{GeV})$ & $\begin{array}{l}\text { number } \\
\text { predicted }\end{array}$ & $\begin{array}{l}\text { probability of } \\
\text { agreement }^{*}(\bar{x})\end{array}$ \\
\hline \multirow[t]{10}{*}{258.69} & 1 & 400 & 34.63 & \\
\hline & 1 & 500 & 14.74 & \\
\hline & 1 & 600 & 7.49 & \\
\hline & 1 & 650 & 5.62 & 2.42 \\
\hline & 1 & 660 & 5.32 & 3.13 \\
\hline & 1 & 670 & 5.07 & 3.89 \\
\hline & 1 & 680 & 4.82 & $4.78 \dagger$ \\
\hline & 1 & 690 & 4.61 & 5.71 \\
\hline & 1 & 700 & 4.39 & 6.86 \\
\hline & 1 & $\infty \quad(Q C D)$ & 0.88 & 90.8 \\
\hline \multirow[t]{10}{*}{219.76} & 2 & 650 & 11.18 & 0.11 \\
\hline & 2 & 660 & 10.67 & 0.16 \\
\hline & 2 & 670 & 10.22 & 0.24 \\
\hline & 2 & 680 & 9.82 & 0.33 \\
\hline & 2 & 690 & 9.45 & 0.44 \\
\hline & 2 & 700 & 9.09 & 0.60 \\
\hline & 2 & 800 & 6.60 & 4.30 \\
\hline & 2 & 810 & 6.43 & $4.89 \dagger$ \\
\hline & 2 & 820 & 6.29 & 5.44 \\
\hline & 2 & $\infty \quad(Q C D)$ & 3.26 & 47.0 \\
\hline
\end{tabular}

* The probabilities are calculated as the area under the likel ihood function $P(\mu)=\exp (-\mu) \mu^{r} / r$ ! with $P(\mu)<P(m)$, where $m$ is the theoretical prediction and $r$ is the observed number.

† A limit on the compositeness scale at $95 \%$ confidence level. 


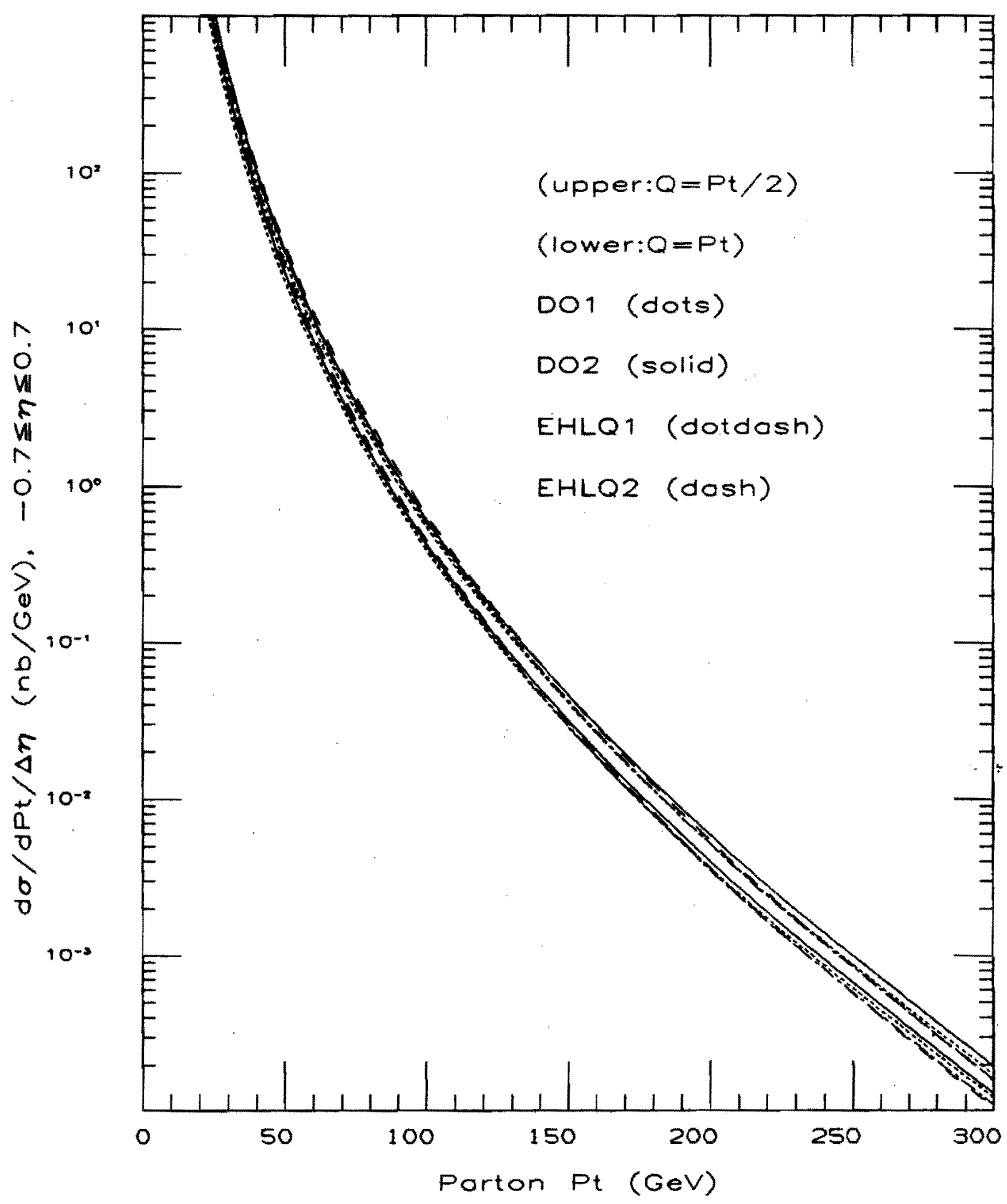

Fig. 75 Parton Pt spectro occording to QCD with different choices of momentum scales \& structure functions. 


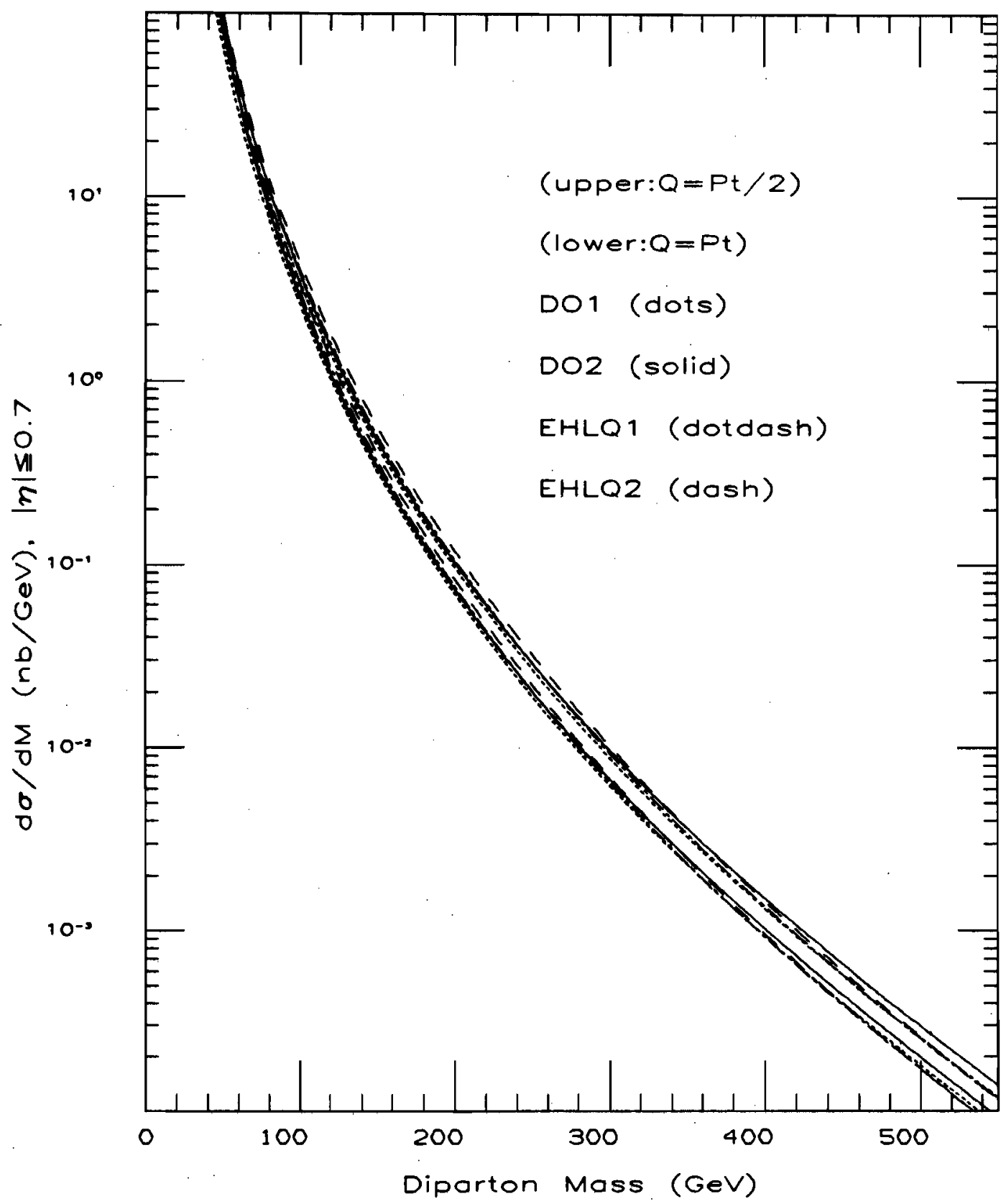

Fig. 76 Diparton mass spectra according to QCD with different choices of momentum scales and structure functions 


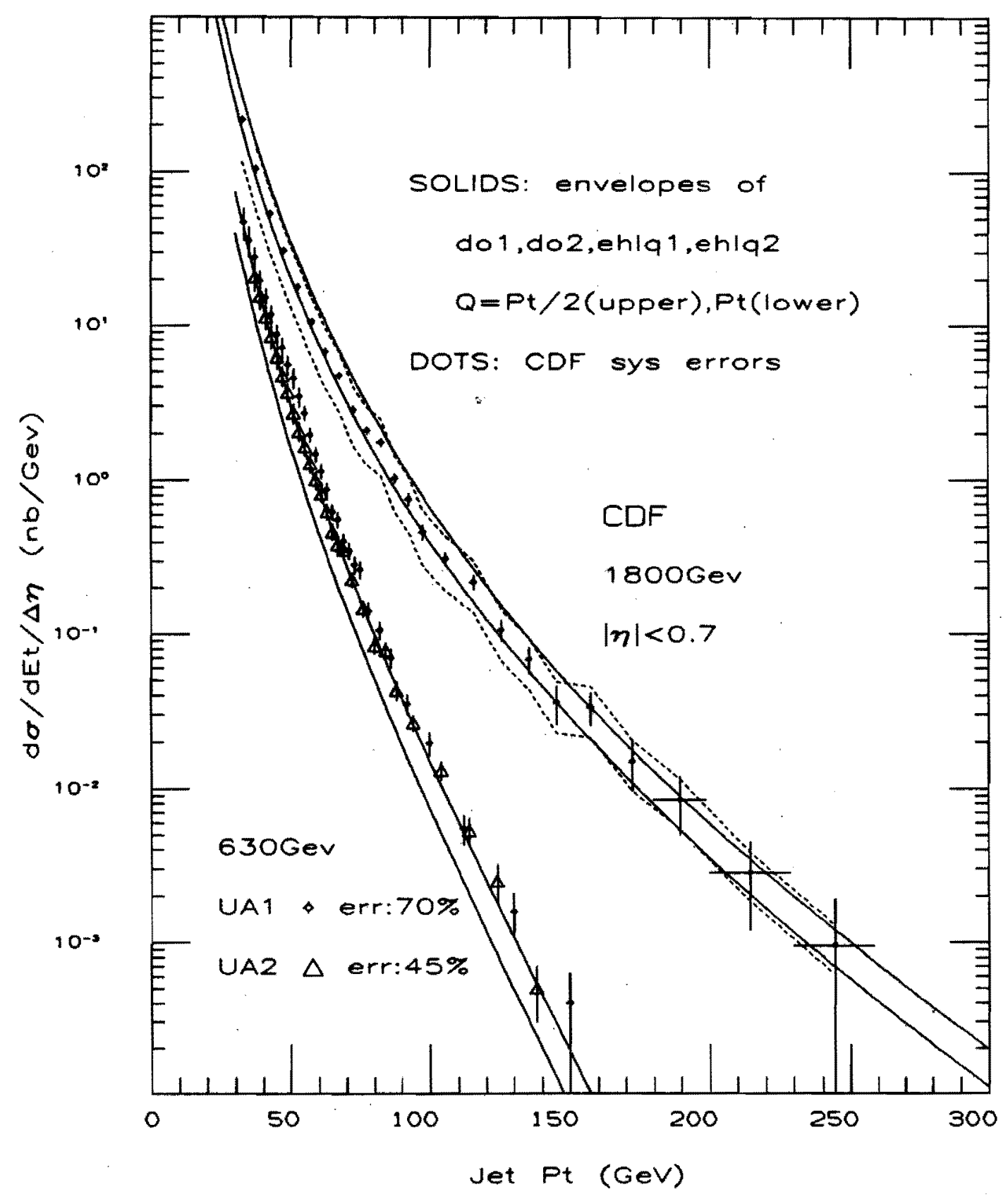

Fig. 78 inclusive jet Pt spectra. 


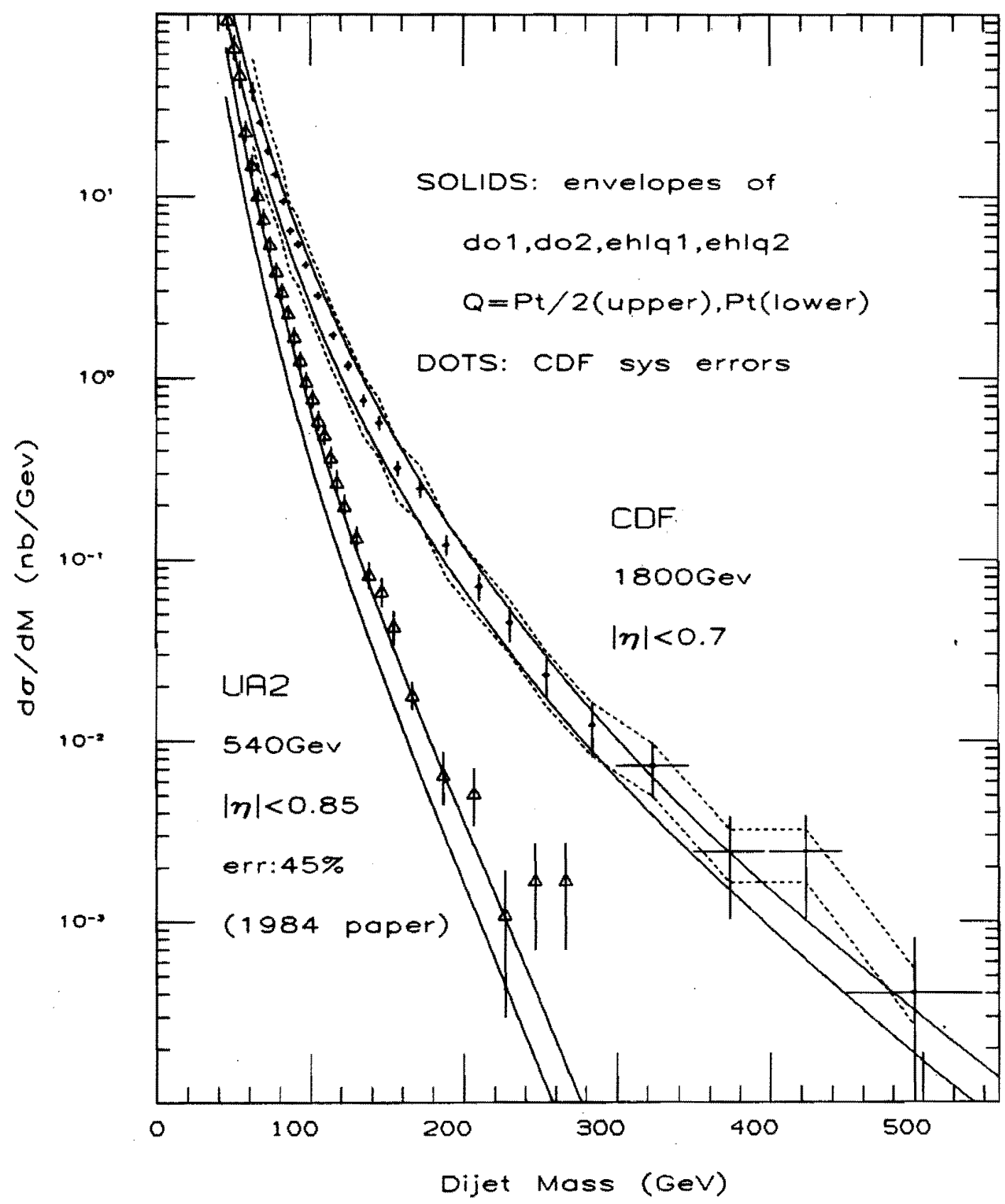

Fig. 79 Dijet invariant mass spectra. 


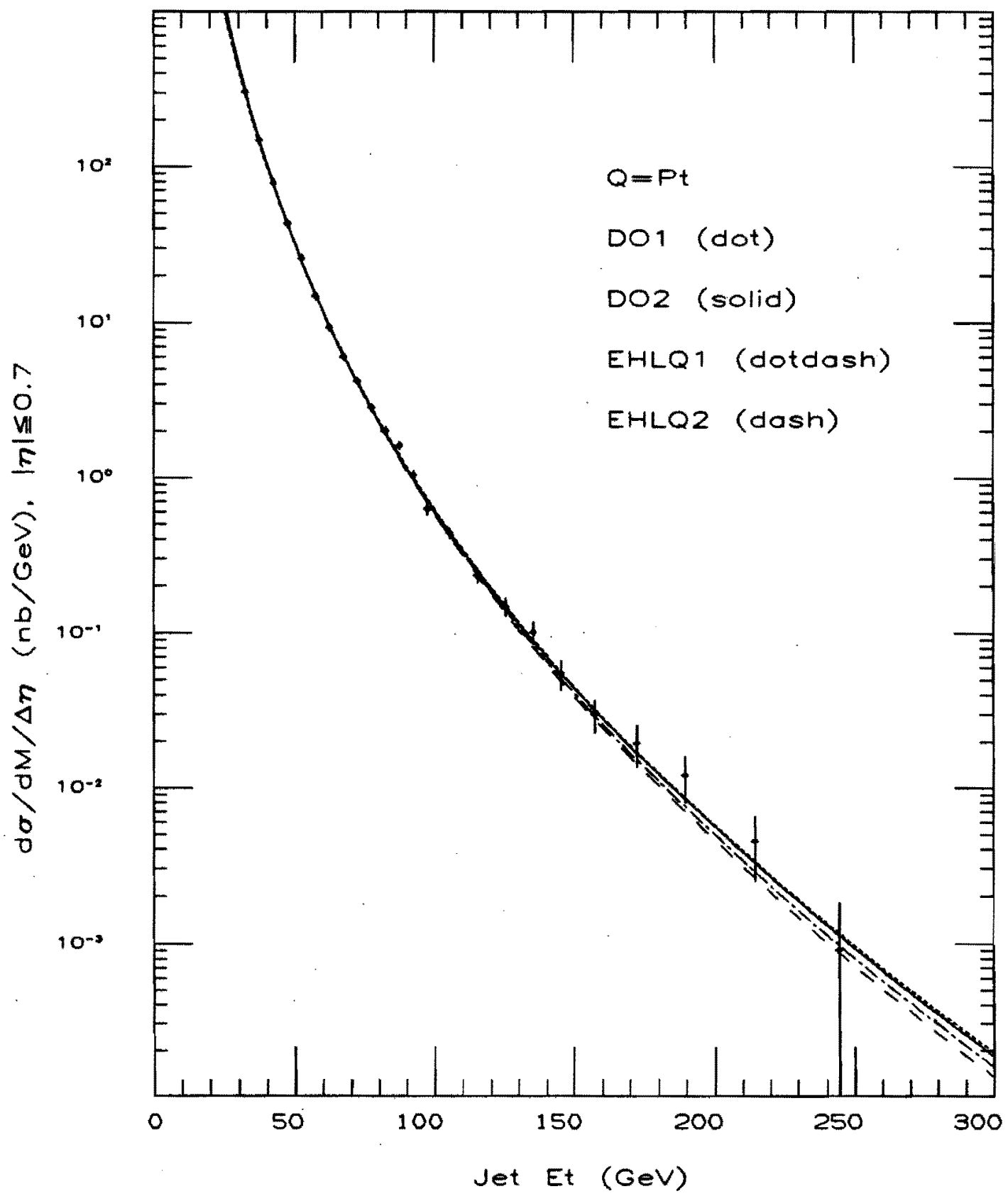

Fig. 80 Unsmeared jet Et spectrum compared with QCD with 4 sets of structure functions. QCD is scaled vertically. to minimize $x^{2}$ (see Table 9.1). 


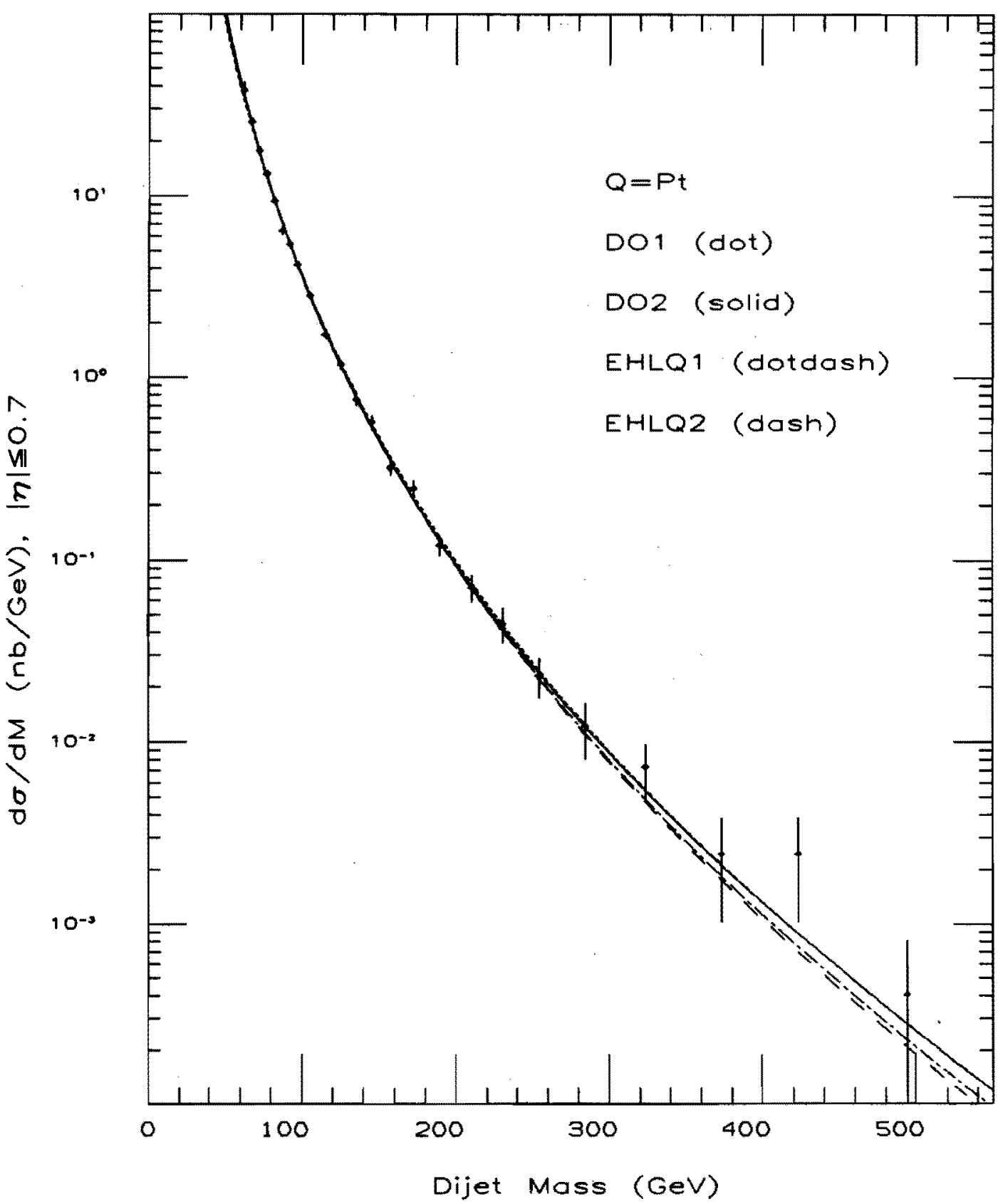

Fig. 81 Unsmeared dijet mass spectrum compared with QCD with 4 sets of structure functions. QCD is scaled vertically to minimize $x^{2}$ (see Table. 9.2). 


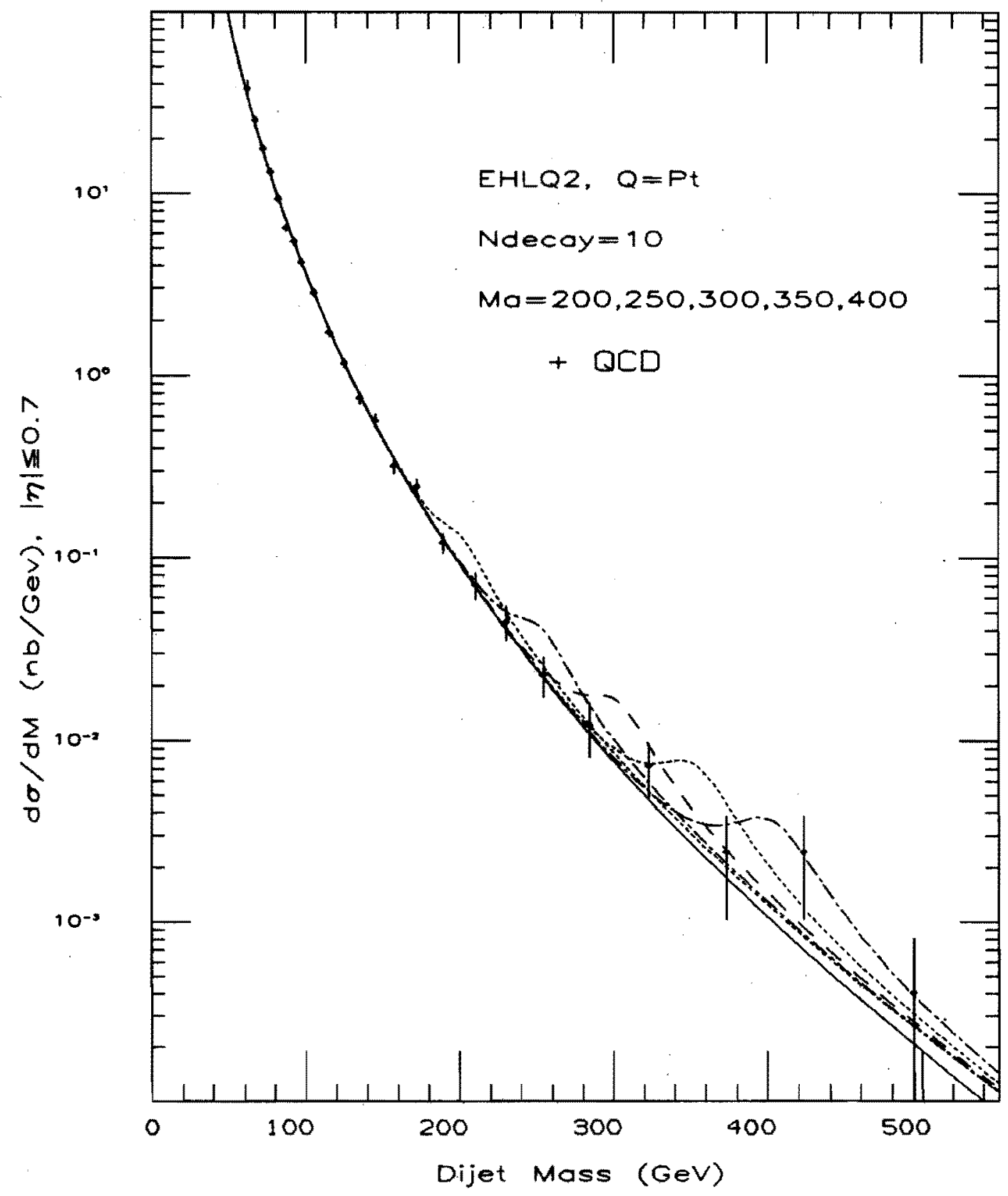

Fig. 82 Unsmeared dijet mass spectrum compared with calculations involving the effects of axigluons. 


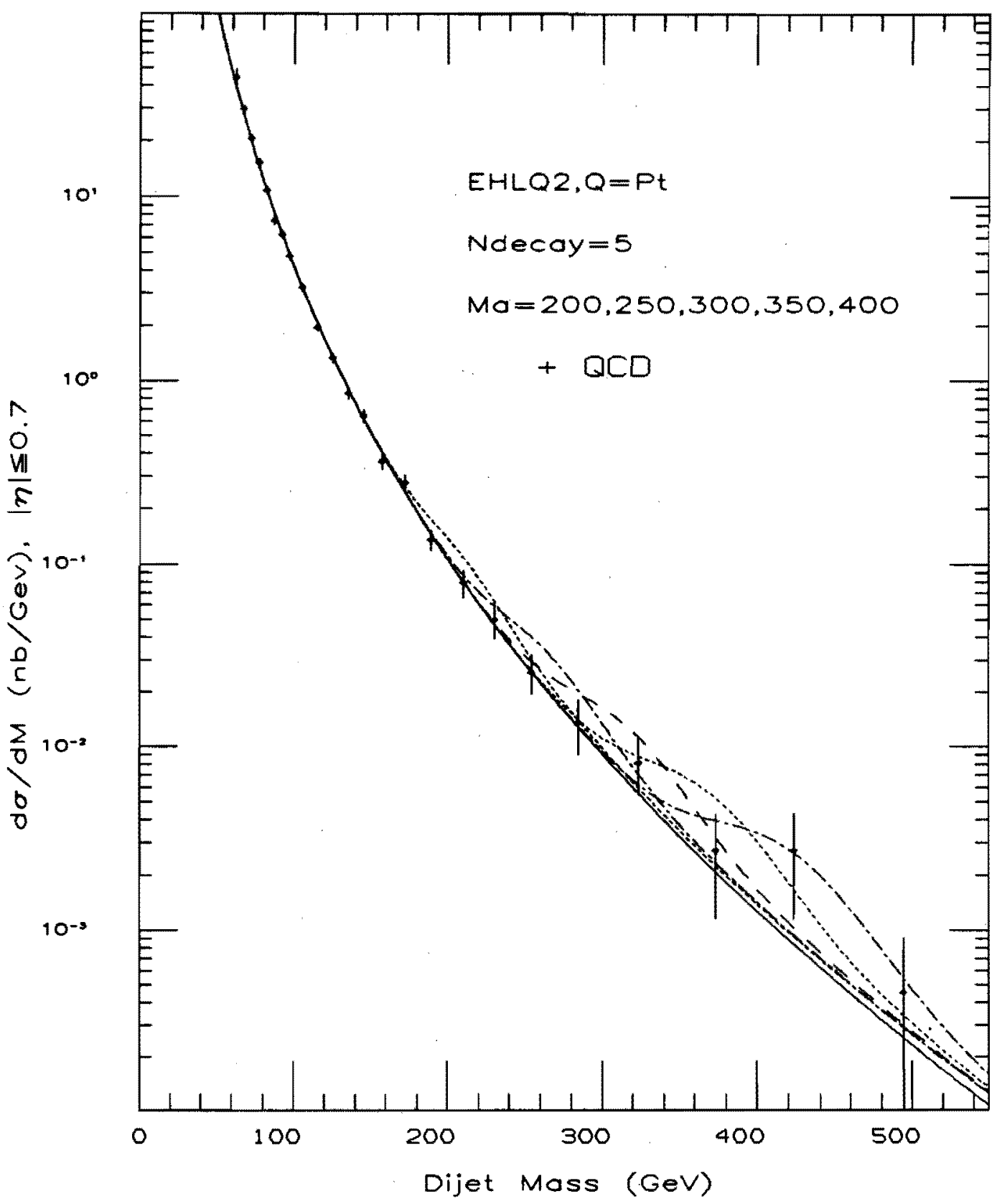

Fig. 83 Smeared dijet mass spectrum compared with calculations involving the effects of axigluons. 


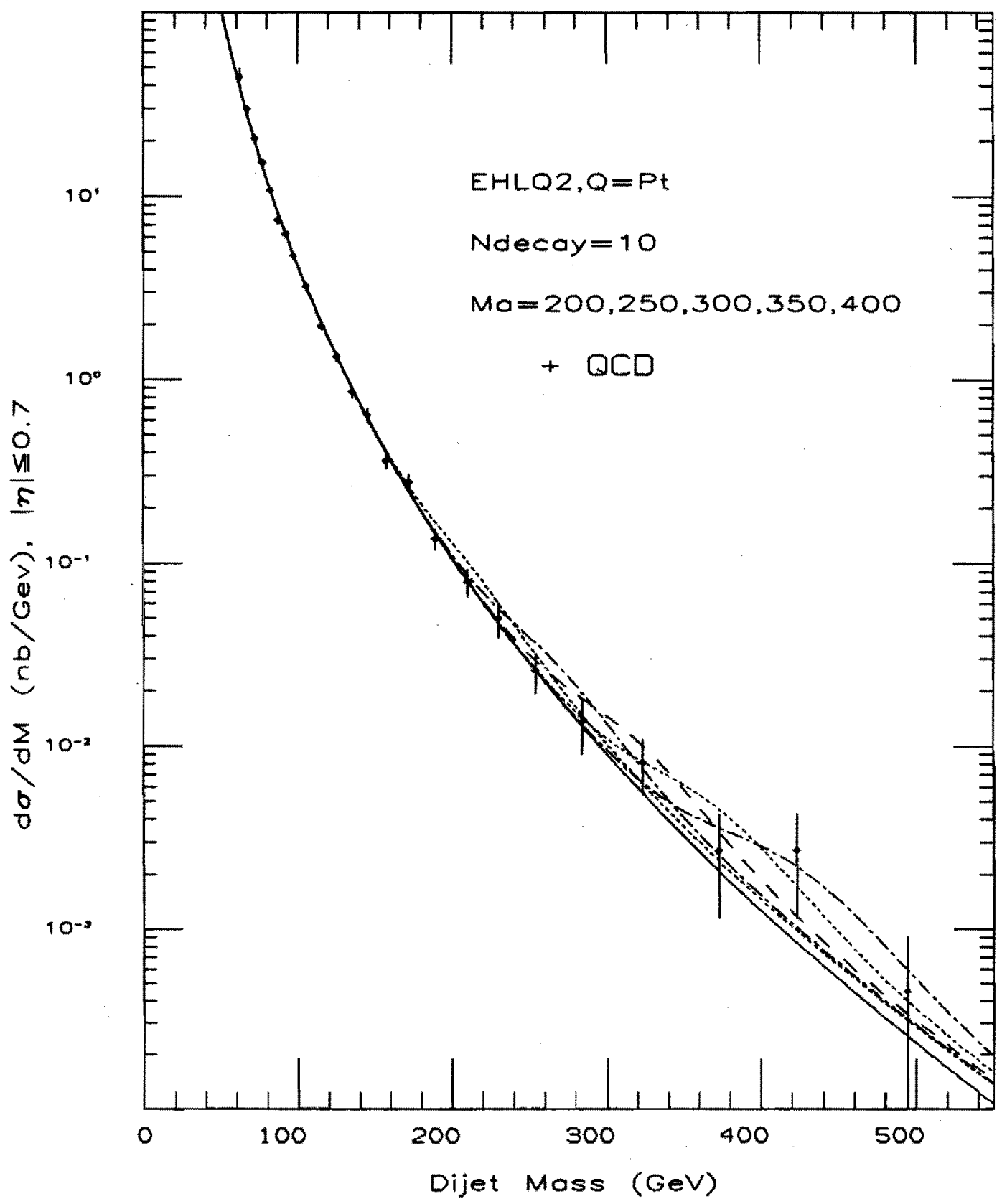

Fig. 84 Smeared dijet mass spectrum compared with calculations involving the effects of axigluons. 


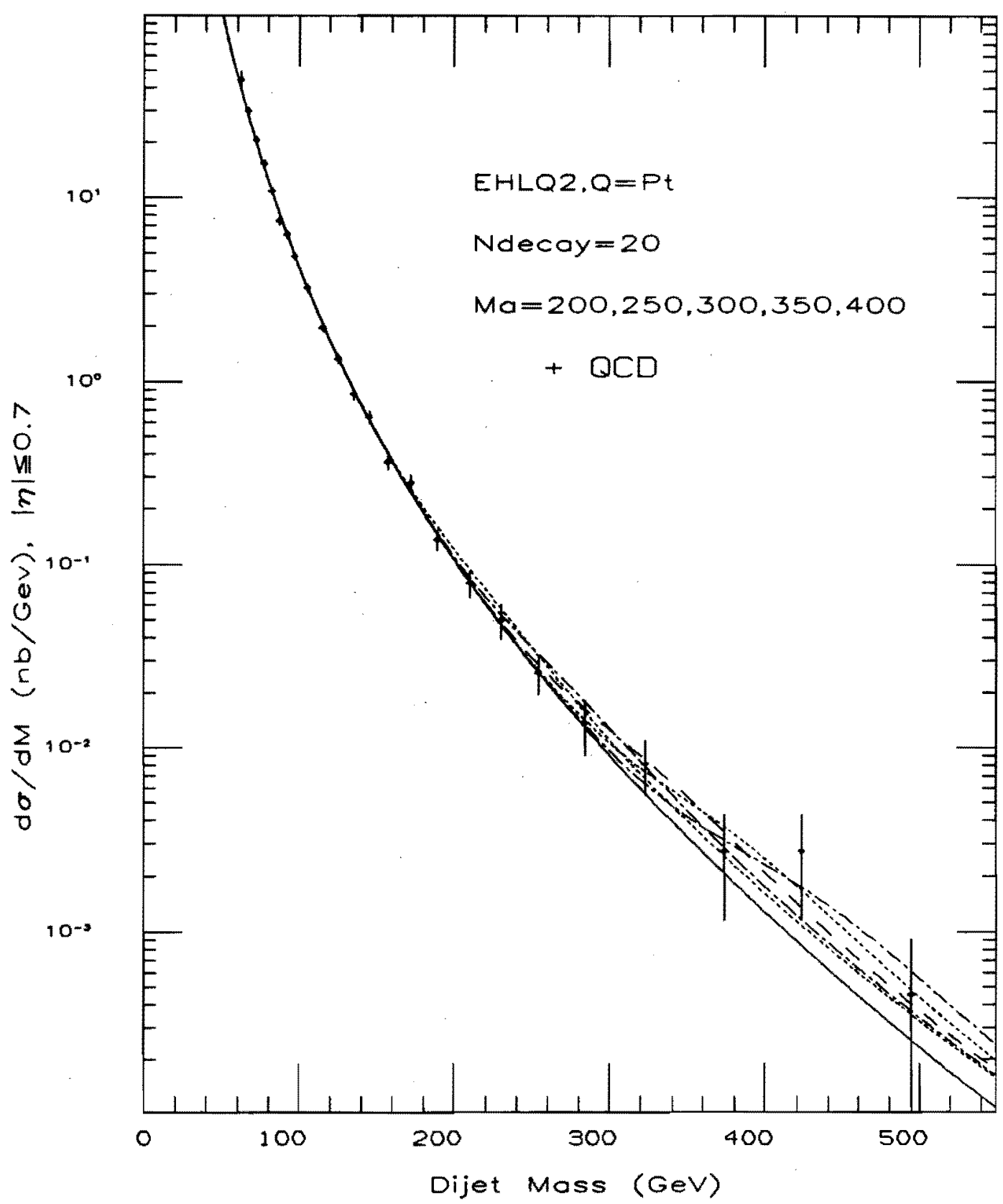

Fig. 85 Smeared dijet mass spectrum compared with calculations involving the effects of axigluons. 


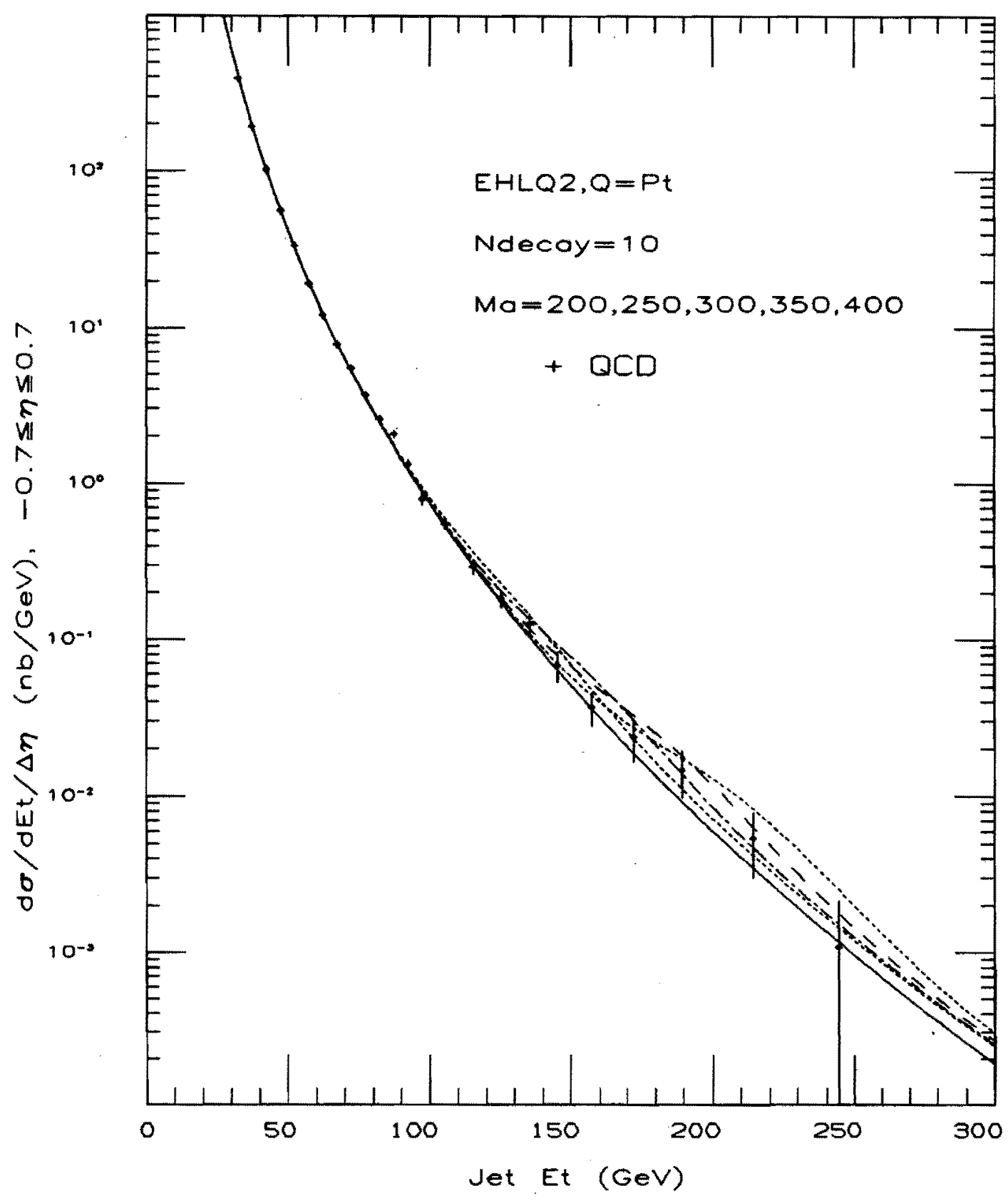

Fig. 86 Smeared jet Et spectrum compared with calculations involving the effects of axigluons. 


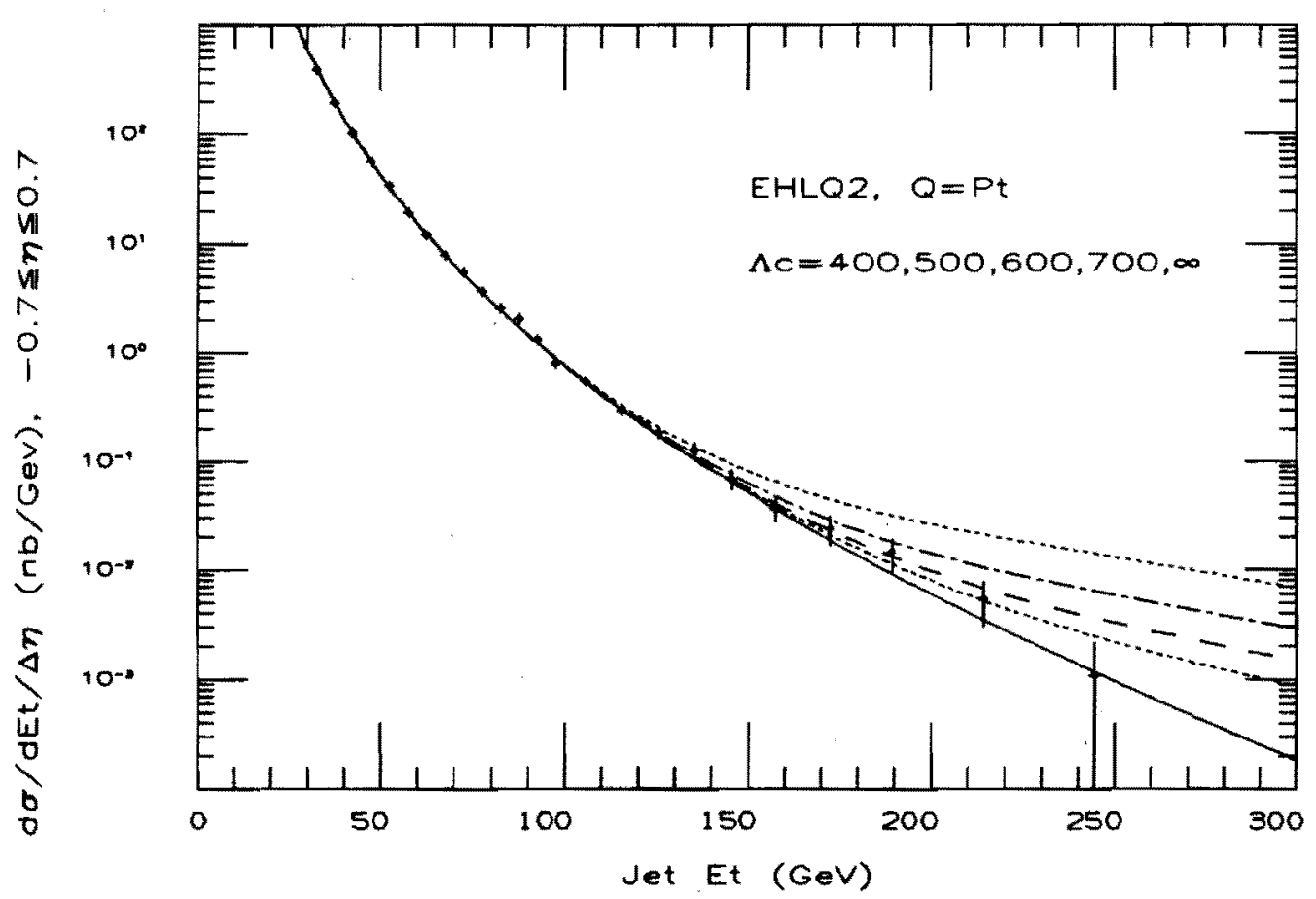

Fig. 87 The smeared jet Et spectro with different compositeness scales. See Table 9.3 for the numbers of jets predicted to be in the region from 258.69 GeV to $\infty$.

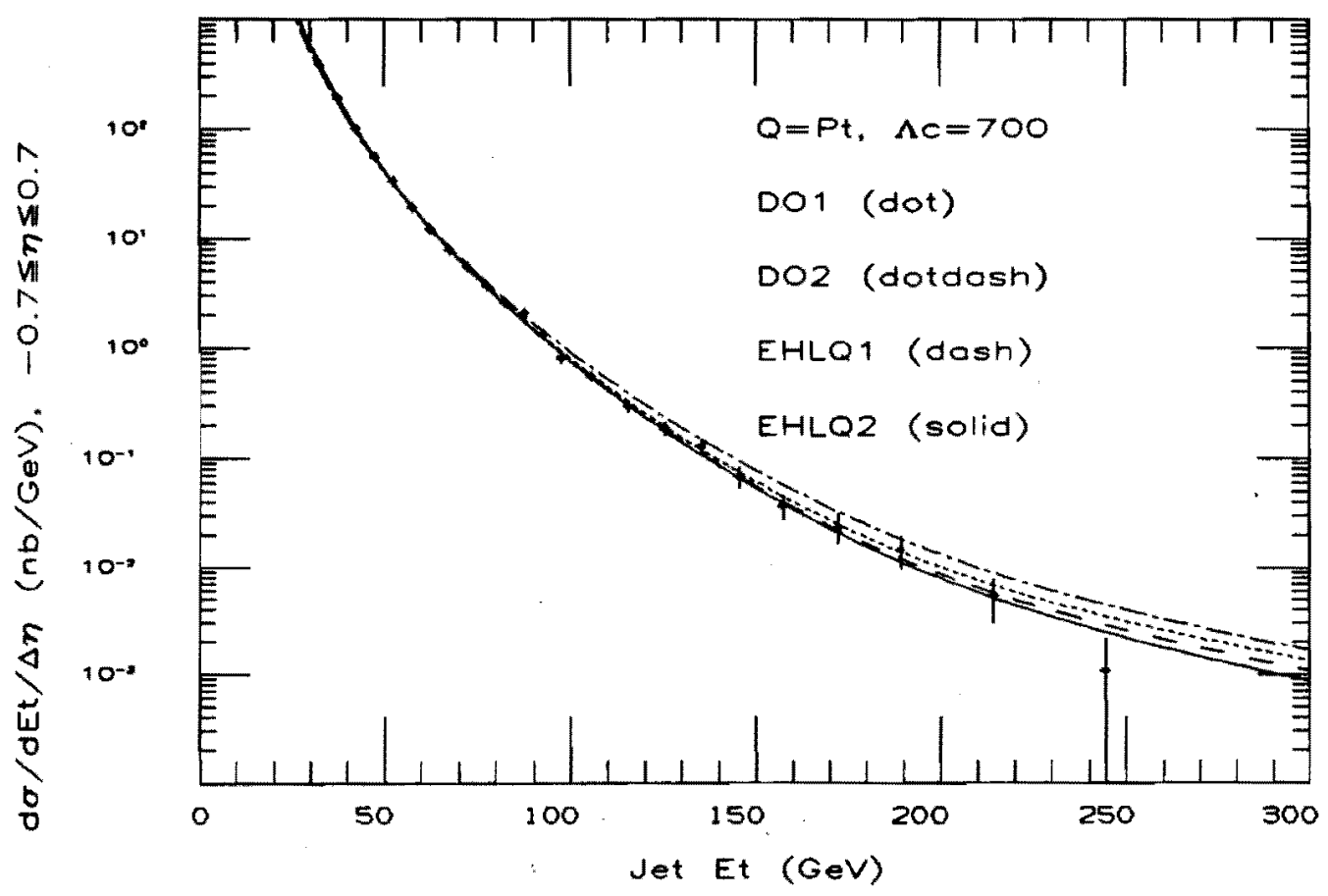

Fig. 88 The smeared jet Et spectra with compositeness with different structure functions. 


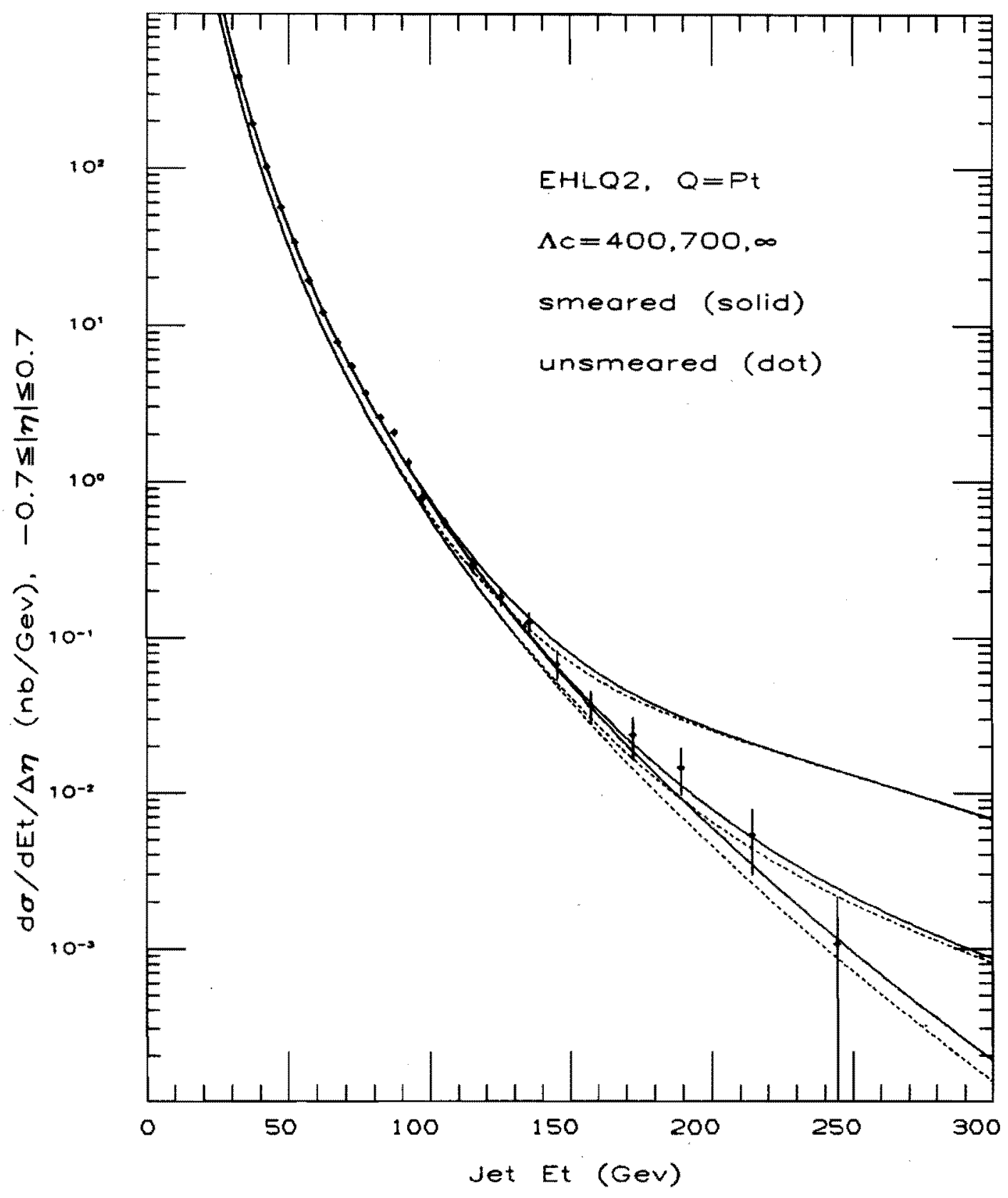

Fig. 89 Comparison of the smeared and unsmeared jet Et spectro with compositeness. 


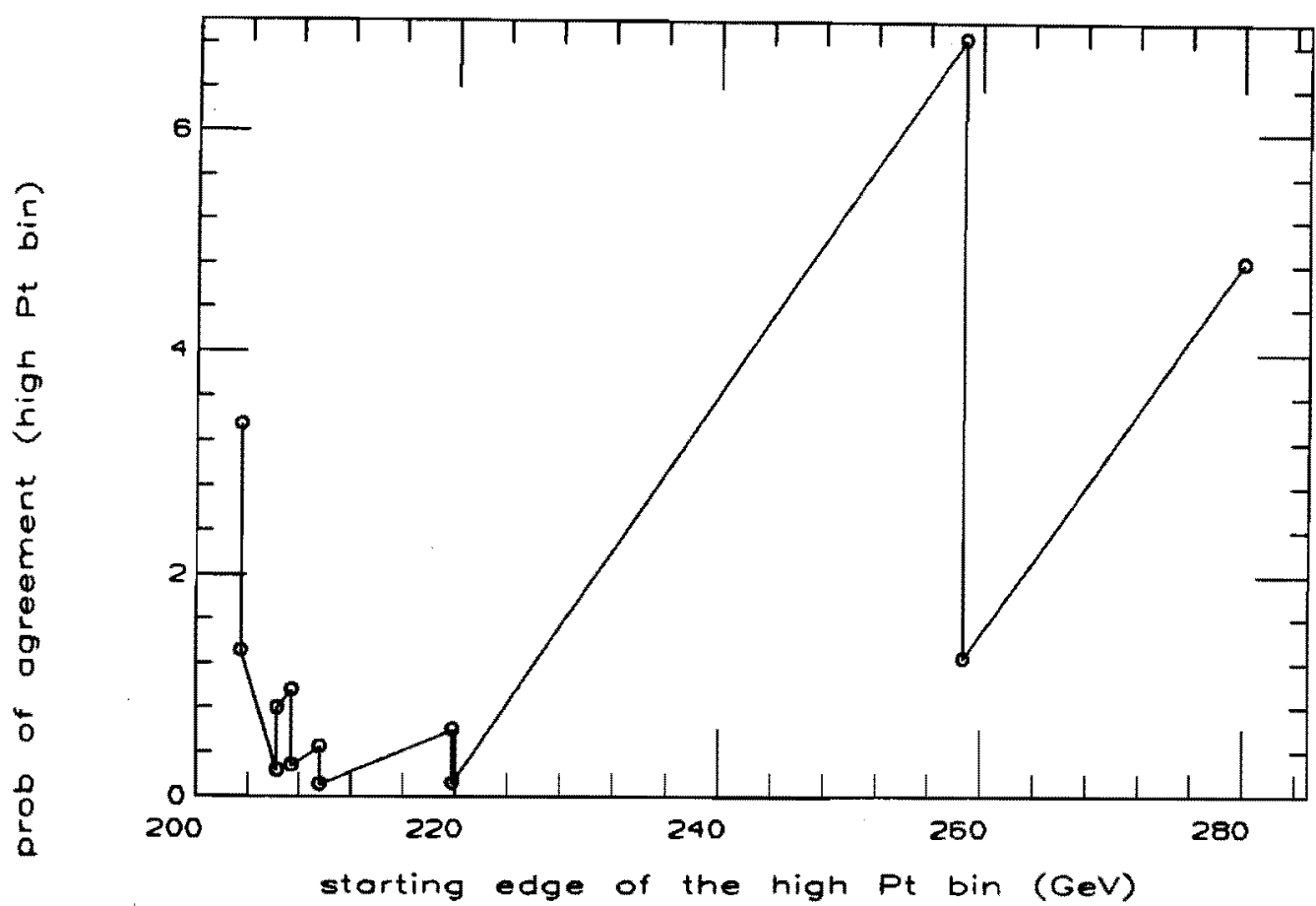

(a)

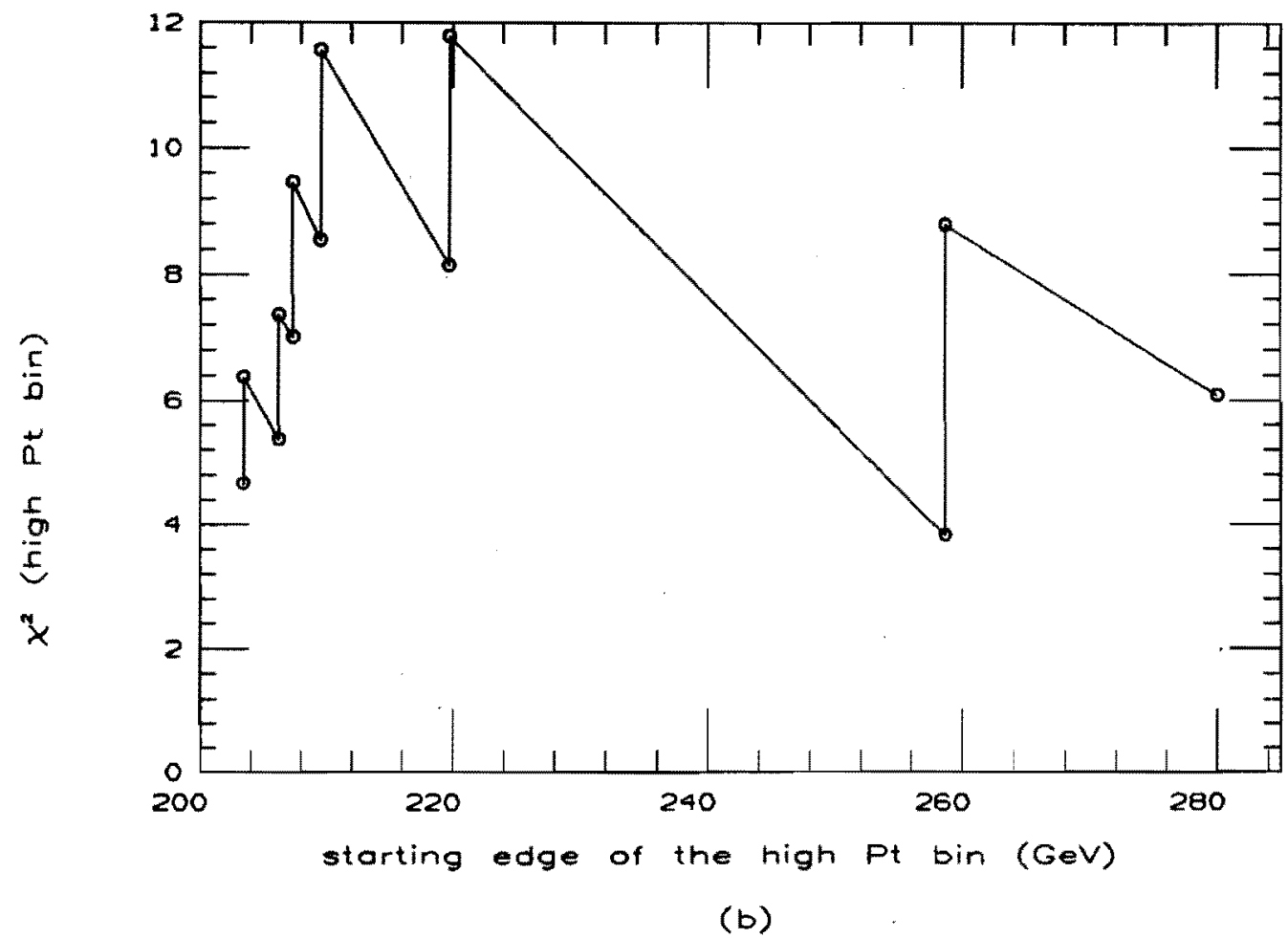

Fig. 90 Comparing compositeness scale $\Lambda c=700 \mathrm{GeV}$ with data for a high pt bin (to $\infty$ ) when the low edge of the bin is varied: a) the prob. of agreement, b) $x^{2}$. 


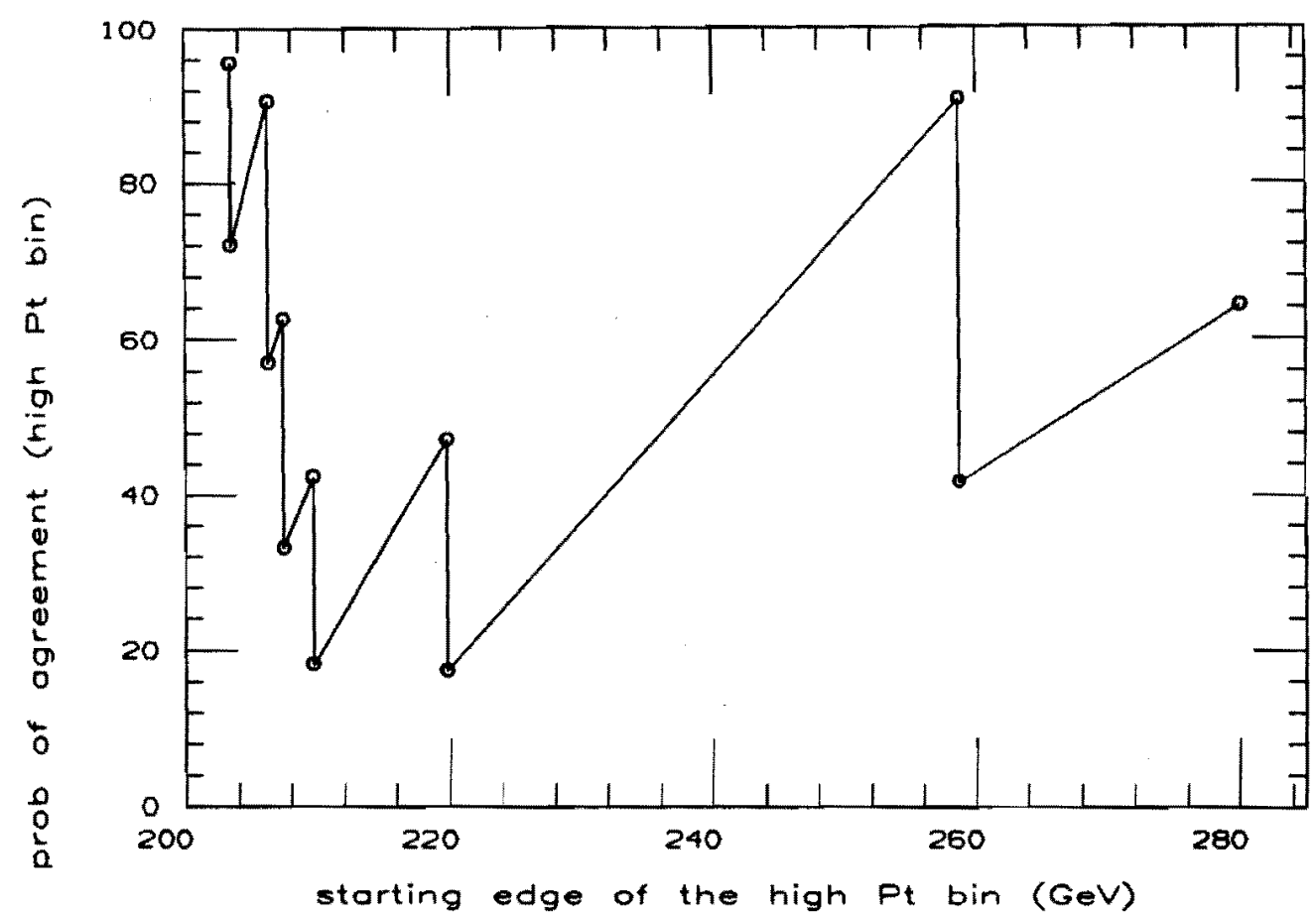

(a)

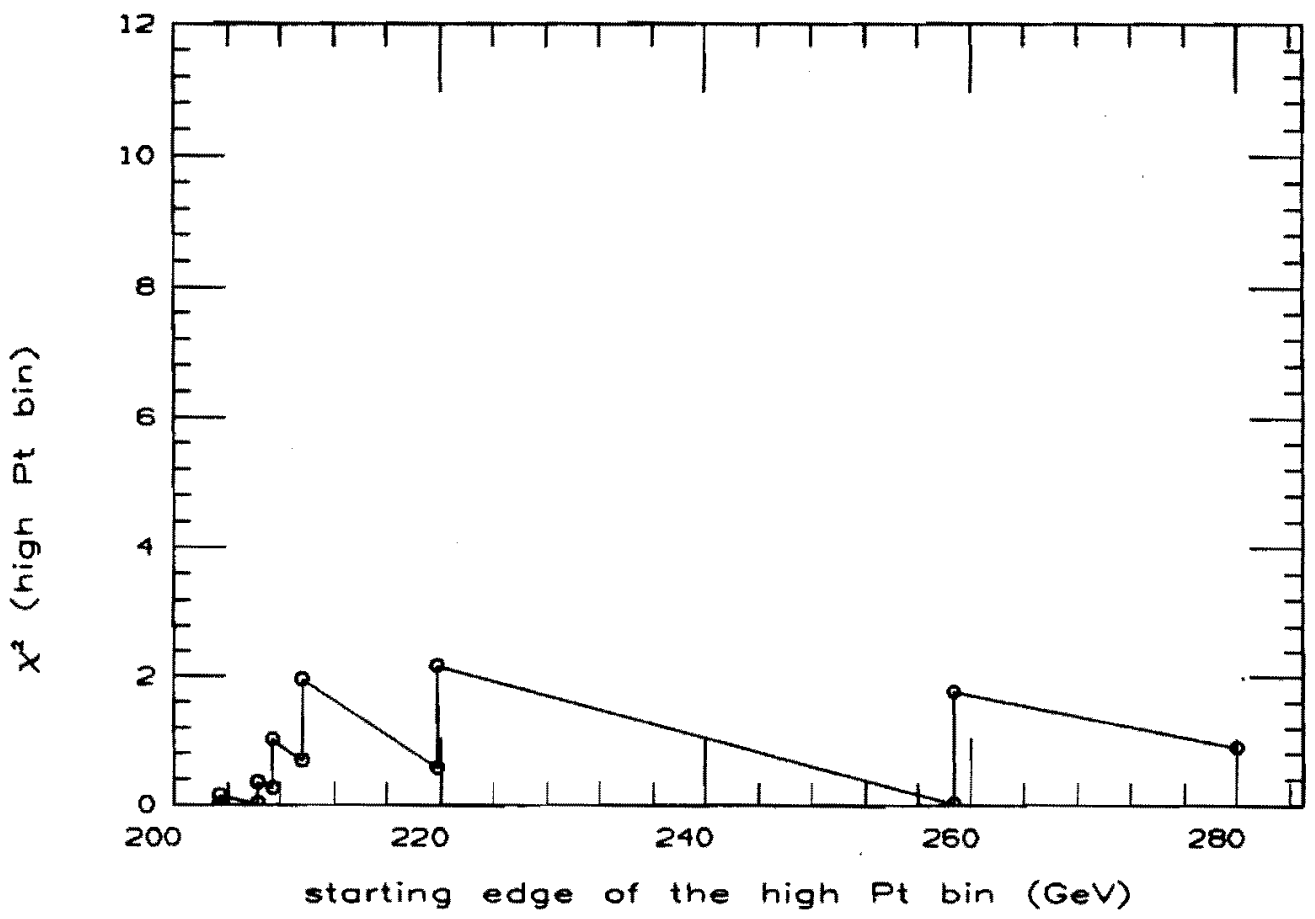

(b)

Fig. 91 Same as Fig. 90, but $O C D$ is compared. 


\section{APPENDIX A}

\section{THE RUNNING COUPLING CONSTANT AND}

STRUCTURE FUNCTIONS IN QCD

\section{A.1 The $Q^{2}$-Evolution of the Strong Coupl ing Constant}

Given the QCD Lagrangian

$$
L=\frac{1}{4} F_{a}^{\mu \nu} F_{a \mu \nu}+i \bar{\phi}_{j} \gamma_{\mu} D_{j k}^{\mu} \phi_{k}-\bar{\phi}_{j} M_{j k} \phi_{k} \text {, }
$$

where

$$
\begin{aligned}
& F_{a}^{\mu \nu}=\partial^{\mu} A_{a}^{\nu}-\partial^{\nu} A_{a}^{\mu}+g f_{a b c} A_{b}^{\mu} A_{c}^{\nu} \\
& D_{j k}^{\mu} \equiv \delta_{j k} \partial^{\mu}-i g\left(T_{a}\right)_{j k} A_{a}^{\mu},
\end{aligned}
$$

the effective coupling $\bar{g}\left(Q^{2}\right)$ can be shown to be arbitrarily small as $Q^{2} \rightarrow$ $\infty$ (i.e. the theory is "asymptotically free"). In the following we will review how this is derived following the argument of Reya [57]. Throughout this appendix, equations marked by (R*.*) are those found in the excellent review of Reya. To give clearer views of the derivations (without going through the jungle), I have kept only the main points and rearranged them, and added some useful comments.

Define $\Gamma_{u}(n A, n \phi)$ to be the $1 P I$ (one particle irreducible) truncated Green's function (with the $n_{A}$ external boson propagators and $n_{p}$ external fermion propagators removed). The theory can be made finite (renormalized) by the subtraction of infinities at some arbitrary Euclidian point $p_{i}{ }^{2}=-\mu^{2}$ (momentum subtraction scheme). The choice of Euclidian $p_{i}{ }^{2}$ 's satisfies the condition of Weinberg's theorem [58] which says: If all external four momenta are deep Euclidian, parameterized as $p_{i}=\lambda k_{i}$, and no partial sum of momenta (other than total sum) vanishes, then the 1PI Green's function grows as $\lambda^{d}$ times polynomials in $\ln \lambda$ to any finite order, where $d$ is the dimension of the Green's function. The renormalized and bare Green's functions are related by 


$$
\Gamma^{(n A, n \phi)}\left(p_{i}, g, \mu\right)=\lim _{\Lambda \rightarrow \infty} Z_{A}\left(g_{0}, \Lambda / \mu\right)^{n A_{Z}}\left(g_{0}, \Lambda / \mu\right)^{n \phi_{\Gamma}} \prod_{u}^{(n A, n \phi)}\left(p_{i}, g_{0}, \Lambda\right)
$$

where $\mathrm{g}=\mathrm{g}\left(\mathrm{g}_{0}, \Lambda / \mu\right)$, and the $Z$ 's are the renormalization constants def ined in such a way that the following expressions are valid

$$
\begin{array}{ll}
\text { boson propagator: } & \left.\Gamma^{(2,0)}\right|_{p^{2}=-\mu^{2}}=\left.Z_{A}^{2} \Gamma_{u}^{(2,0)}\right|_{p^{2}=-\mu^{2} \equiv-g_{\mu \nu}^{2} p+p_{\mu} p_{\nu}} \\
\text { fermion propagator: } & \left.\left.\Gamma^{(0,2)}\right|_{p^{2}=-\mu^{2}}{ }^{2} Z_{\psi}^{2} \Gamma_{u}^{(0,2)}\right|_{p^{2}=-\mu^{2}} ^{2} \equiv \gamma \cdot p \quad \text { (R3.12) } \\
\text { coupling: } & \left.\Gamma^{(1,2)}(0, p,-p)\right|_{p^{2}=-\mu^{2}}=\left.Z_{A} Z_{u}^{2} \Gamma_{u}^{(1,2)}\right|_{p}{ }^{2}=-\mu^{2} \equiv g .
\end{array}
$$

The renormalization group (RG) equation is then derived from the fact that $\mathrm{d} \Gamma_{\mathrm{u}} / \mathrm{d} \mu=0$ :

$$
\left[\mu \frac{\partial}{\partial \mu}+\beta(g) \frac{\partial}{\partial g}+n_{A} \gamma_{A}(g)+n_{\phi} \gamma_{\psi}(g)\right] \Gamma^{(n A, n \phi)}\left(p_{i}, g, \mu\right)=0
$$

where

$$
\begin{aligned}
& \rho(g) \equiv \lim _{\Lambda+\infty} \mu \frac{\partial}{\partial \mu} g\left(g_{0}, \Lambda / \mu\right) \\
& \gamma_{j}(g) \equiv-\lim _{\Lambda+\infty} \mu \frac{1}{Z_{j}} \frac{\partial}{\partial \mu} Z_{j}\left(g_{0}, \Lambda / \mu\right) .
\end{aligned}
$$

However, the dependence on $\mu$ can be translated into the dependence on $p_{i}$. If we scale $p_{i}+e^{t} p_{i}$, then

$$
\left[-\frac{\partial}{\partial t}+\rho(g) \frac{\partial}{\partial g}+d+n_{A} \gamma_{A}(g)+n_{\phi} \gamma_{\psi}(g)\right] \Gamma(n A, n \phi)\left(e^{t} p_{i}, g, \mu\right)=0
$$

where $d$ is the dimension of $\Gamma$. The homogeneous part of this equation (without $d$ and $\gamma^{\prime} s$ ) is solved by $\phi(\bar{g}(g, t))$ if $\bar{g}$ satisfies

$$
\frac{\mathrm{d} \overline{\mathrm{g}}(\mathrm{g}, \mathrm{t})}{\mathrm{dt}}=\rho(\overline{\mathrm{g}}) \text {. }
$$

If we define $\gamma \equiv n \gamma_{A}+n \gamma_{\phi}$, then the general solution of (3.18) is given by $\Gamma\left(e^{t} p, g\right)=\Gamma(p, \bar{g}) \exp \left[\int_{0}^{t} \gamma\left(\bar{g}\left(g, t^{\prime}\right)\right) d t^{\prime}\right]=\Gamma(p, \bar{g}) \exp \left[\int_{g}^{\bar{g}} \frac{\gamma\left(g^{\prime}\right)}{\beta\left(g^{\prime}\right)} d g^{\prime}\right]$ 
Now the power of the $R G$ equations is related to the fact that if there is a value of $\mu$ for which $g^{2} / 4 x^{2} \ll<1, \quad \beta(g)$ and $\gamma(g)$ can be evaluated from the lowest order diagrams (for $\mathrm{SU}(3)_{c}$ and $\mathrm{N}_{f}$ flavors):

$$
\begin{aligned}
& f(g)=-\frac{1}{16 \pi^{2}}\left[\frac{33}{3}-\frac{2}{3} N_{f}\right] g^{3} \equiv-b g^{3} \\
& \gamma_{A}(g)=\frac{1}{16 \pi^{2}}\left[\frac{13}{2}-\frac{2}{3} N_{f}\right] g^{2}+0\left(g^{4}\right), \quad \gamma_{f}=0+0\left(g^{4}\right) .
\end{aligned}
$$

Inserting $\beta$ of (4.5) into (3.23), we finally have

$$
\bar{g}(t)^{2}=\frac{q^{2}}{1+2 b g^{2} t}=\frac{g^{2}}{1+b g^{2} \ln \left(Q^{2} / \mu^{2}\right)} \equiv \frac{1}{b \ln \left(Q^{2} / A^{2}\right)},
$$

which implies $g^{2}(t) \rightarrow 0$ for $t \rightarrow \infty$ if $N_{f}<16$. If $\gamma(g) \equiv c g^{2}$ (see Eq. A12), Eq. A11 and Eq. A10 give

$$
\Gamma\left(e^{t} p, g\right)=\Gamma(p, \bar{g})\left[g^{2} / \bar{g}^{2}\right]^{c / 2 b} \text {. }
$$

\section{A.2 The $Q^{2}$-Evolution of the Structure Functions}

Three ways of deriving the evolution of structure functions will be discussed (see Reya [57]): by a formal field approach, by integrating the famous Altarelli-Parisi (A-P) equations, and by summing up the BetheSalpeter (B-S) ladders (the diagrams of leading logarithms). As in the case of Bjorken scaling, the QCD evolution was first derived by the field approach, which then inspired (and justified) a simple alternative, the A-P equations. But the field method, though sacred, has rather limited use (besides being the most difficult) as there are processes other than deep inelastic scattering where the OPE (operator product expansion) doesn't apply. The A-P equations are the equations of structure functions, which are the only use of them. The method of the B-S ladder, however, is based on a fact that the ladder diagrams (in axial gauge) give all leading logs, and thus is the most useful tool for various QCD applications other than structure functions (e.g. initial and final state radiations, see Appendix B).

\section{A.2.1 The Field Theory Derivation}


The field approach utilizes Wilson's operator product expansion (OPE) [59] and renormalization group (RG) equations. The OPE factors out the perturbatively calculable part (hot part: the Wilson coefficients $C^{n}$ ) and the incalculable bound state (cold part: expectation values $\left\langle N\left|0^{n}\right| P\right\rangle$ of Wilson operators $0^{n}$ ). The RG equations for $C^{n}$ give the same results as summing up all the leading log diagrams (which would be equivalent to the Bethe-Salpeter ladders in axial gauge). Effectively the OPE identifies the leading diagrams and RG does the summation.

Define the deep inelastic scattering amplitude $W_{\mu \nu}$ as in Fig. 92

$$
\begin{aligned}
W_{\mu \nu} & =\frac{1}{4 \pi} \sum_{X}(2 \pi)^{4} \delta^{4}\left(p+q-p_{X}\right)\left\langle p\left|J_{\mu}^{+}(0)\right| X\right\rangle\left\langle X\left|J_{\nu}(0)\right| p\right\rangle \\
& =\frac{1}{4 \pi} \int d z^{4} e^{i q^{\cdot} z}\left\langle p\left|J_{\mu}^{+}(z) J_{\nu}(0)\right| p\right\rangle=\frac{1}{4 \pi} \int d z^{4} e^{i q \cdot z}\left\langle p\left|\left[J_{\mu}^{+}(z), J_{\nu}(0)\right]\right| p\right\rangle \\
& \equiv\left(-g_{\mu \nu}+\frac{q_{\mu} q_{\nu}}{q^{2}}\right) W_{1}+\frac{1}{m_{N}^{2}}\left(p_{\mu}-\frac{p^{\cdot} q}{q^{2}} q_{\mu}\right)\left(p_{\nu}-\frac{p^{\cdot} q}{q^{2}} q_{\nu}\right) W_{2}-i \frac{\epsilon \nu a \beta^{p^{a}} q^{\beta}}{2 m_{N}^{2}} W_{3} .
\end{aligned}
$$

In the deep inelastic region $\left(Q^{2} \equiv-q^{2}, p \cdot q,(p+q)^{2} \gg m_{N}^{2}\right.$ with $x \equiv Q^{2} / 2 p \cdot q$ fixed) the dimensionless $W_{i}$ approach nontrivial functions of only one variable (Bjorken scaling)

$$
W_{1}\left(p \cdot q, Q^{2}\right) \rightarrow F_{1}(x), \quad \frac{p^{\prime} q}{m_{N}^{2}} W_{i=2,3}\left(p \cdot q, Q^{2}\right) \rightarrow F_{i}(x)
$$

The scaling functions $F_{i}$ can be shown to give (in the parton model) the parton distributions as in Eq. 9 (chapter 1).

The amplitude $W_{\mu \nu}$ is related to the virtual Compton amplitude

$$
\begin{aligned}
T_{\mu \nu} & \equiv \mathrm{i} \int d^{4} z e^{i q^{\cdot} z}\left\langle p\left|T\left(J_{\mu}^{+}(z) J_{\nu}(0)\right)\right| p\right\rangle \\
& =i \int d^{4} z e^{i q \cdot z} \theta\left(z_{0}\right)\left\langle p\left|\left[J_{\mu}^{+}(z), J_{\nu}(0)\right]\right| p\right\rangle
\end{aligned}
$$

via the optical theorem

$$
W_{\mu \nu}=\frac{1}{2 \pi} \operatorname{Im} T_{\mu \nu} .
$$

In the limit $z^{2} \rightarrow 0, T_{\mu \nu}$ can be expanded in terms of Wilson operators (examples can be found in Eq. A30 and A32) as 


$$
\begin{aligned}
& T=\sum_{i, n} \int d^{4} z e^{i q \cdot z} c_{i}^{n}\left(z^{2}\right) z_{\mu 1} \cdots z_{\mu n}\left\langle p\left|0_{i}^{\mu_{1} \cdots \mu n}(0)\right| p\right\rangle \\
& =\sum_{i, n} 2 q_{\mu_{1}} \cdots 2 q_{\mu n} \frac{\partial^{n}}{\partial\left(i q^{2}\right)^{n}} \int d^{4} z e^{i q^{\cdot} z} c_{i}^{n}\left(z^{2}\right)\left\langle p\left|0_{i}^{\mu_{1} \cdots \mu n}(0)\right| p\right\rangle \\
& \equiv \sum_{i, n}\left(Q^{2} / 2\right)^{-n} q_{\mu 1} \cdots q_{\mu n} C_{i}^{n}\left(Q^{2}\right)\left\langle p\left|0_{i}^{\mu 1} \cdots \mu n(0)\right| p\right\rangle \\
& =\sum_{i, n} C_{i}^{n}\left(Q^{2}\right) x^{-n} A_{i}^{n}+O\left[x^{-n+2} m^{2} / Q^{2}\right],
\end{aligned}
$$

where $A_{i}^{n}$ are defined through

$$
\left\langle p\left|0_{i}^{\mu_{1} \cdots \mu n}(0)\right| p\right\rangle=A_{i}^{n}\left(p^{\mu 1} \cdots p^{\mu n}-m^{2} g^{\mu 1} \mu^{\mu 2} p^{\mu 3} \cdots\right)
$$

The $C_{i}^{n}$ in Eq. A19 can be isolated by taking the $x^{n-1}$ moment and integrating a contour $C$ around the physical cut $[-1,1]$. The discontinuity is $W$ according to the optical theorem Eq. A18, and we have

$$
\begin{aligned}
4 \int_{0}^{1} d x x^{n-1} W\left(x, Q^{2}\right) & =\frac{1}{2 \pi i} \int_{C} d x x^{n-1} T\left(x, Q^{2}\right)=\sum_{i} C_{i}^{n}\left(Q^{2}\right) A_{i}^{n} . \\
& =\int_{0}^{1} d x x^{n-2} F\left(x, Q^{2}\right)
\end{aligned}
$$

with $F=x F_{1}, F_{2}$, or $x F_{3}$ (see Eq. A16). Both $T_{\mu \nu}$ and $0_{i}^{n}$ must satisfy the RG equations:

$$
\begin{aligned}
& \left(\mu \frac{\partial}{\partial \mu}+\beta \frac{\partial}{\partial g}+2 \gamma_{\phi}\right) c_{i}^{n}\left(Q^{2} / \mu^{2}, g(\mu)\right)\left\langle\phi\left|0_{i}^{n}\right| \phi\right\rangle=0 \\
& \left(\mu \frac{\partial}{\partial \mu}+\beta \frac{\partial}{\partial g}+2 \gamma_{\phi}+\gamma_{0}^{n}\right)\left\langle\phi\left|0_{i}^{n}\right| \phi\right\rangle=0,
\end{aligned}
$$

where $\phi$ denotes $A_{a}^{\mu}$ or $\phi_{k}$ and we have used the fact that for a conserved current $J, \gamma_{J}=0$. The normalization of $0_{i}^{n}$ is specified such that

$$
\left\langle\phi\left|0_{i}^{n}\right| \phi\right\rangle=1+g^{2} b_{i}^{n} \ln \left(-p^{2} / \mu^{2}\right)+0\left(g^{4}\right) \text {. }
$$

Substituting Eq. A24 into Eq. A22 and A23 we have the RG equation for the Wilson coefficients

$$
\left(\mu \frac{\partial}{\partial \mu}+\beta \frac{\partial}{\partial g}-\gamma_{0} n\right) C_{i}^{n}\left(Q^{2} / \mu^{2}, g(\mu)\right)=0 .
$$


If there are several operators of type $i$ (with the same internal quantum numbers and same Lorentz structure), then the 0 's will mix under renormalization and we have

$$
\sum_{j}\left[\delta_{i j}\left(\mu \frac{\partial}{\partial \mu}+\beta \frac{\partial}{\partial g}\right)-\gamma_{i j}^{n}\right] c_{j}^{n}\left(Q^{2} / \mu^{2}, g(\mu)\right)=0 .
$$

However, if $\boldsymbol{\gamma}_{i j}^{n}$ can be diagonalized, the solutions are similar to that of Eq. A25 which is given by (see Eq. A10)

$$
\begin{aligned}
C_{i}^{n}\left(Q^{2} / \mu^{2}, g\left(\mu^{2}\right)\right) & =C_{i}^{n}\left(1, \bar{g}\left(Q^{2}\right)\right) \exp \left[-\int_{0}^{(1 / 2) \ln \left(Q^{2} / \mu^{2}\right)} \gamma_{0_{i}^{n}}\left(\bar{g}\left(t^{\prime}\right)\right) d t^{\prime}\right] . \\
& =C_{i}^{n}\left(1, \bar{g}\left(Q^{2}\right)\right) \exp \left[-\int_{g}^{\bar{g}}\left[\gamma_{0} n\left(g^{\prime}\right) / \rho\left(g^{\prime}\right)\right] d g^{\prime}\right] .
\end{aligned}
$$

Since the $Q^{2}$-dependence of $C_{i}^{n}\left(1, \bar{g}\left(Q^{2}\right)\right)$ is small and the $A_{i}^{n}$ in Eq. A21 are independent of $Q^{2}$, the Mellin moments of structure functions (Eq. A21) have the $Q^{2}$-dependent form (see Eq. A14, but note the different signs before $\gamma^{\prime}$ s in Eq. A8 and Eq. A25)

$$
\left\langle F_{i}\left(Q^{2}\right)\right\rangle_{n}=\left\langle F_{i}\left(Q_{0}^{2}\right)\right\rangle_{n}\left[a\left(Q_{0}^{2}\right) / a\left(Q^{2}\right)\right]^{-c_{i} / 2 b},
$$

where we have used $\beta(g)=-b g^{3}$ and $\tau_{0}{ }^{n}=c_{i} g^{2}$ (see Eq. A11 and A12).

Now we need to specify the Wilson operators and calculate $\gamma_{0}$. From dimensional analysis, we have

$$
c_{i}^{n}\left(z^{2}\right)-\left(1 / z^{2}\right)^{\left[2 d_{j}-\left(d_{0 i}-n\right)\right] / 2} \text { as } z^{2} \rightarrow 0,
$$

where $d_{j}$ and $d_{0 i}$ are the mass dimensions of the current and the Wilson operator respectively. Thus the most singular terms are those with minimum twist ( $\equiv$ dimension - spin), corresponding to the diagrams in Fig. 93(a) and Fig. 94. Examples of higher twist terms are given in Fig. 93(b). The dominating operators are

$$
\begin{aligned}
& 0_{N S}^{n}=\frac{i^{n-1}}{n !}\left[\bar{\psi} \gamma^{\mu 1} D^{\mu 2} \cdots D^{\mu n} \lambda_{a}+\text { perm. }\right] \\
& 0_{F}^{n}=\frac{i^{n-1}}{n !}\left[\bar{\psi} \gamma^{\mu 1} D^{\mu 2} \cdots D^{\mu n} \phi+\text { perm. }\right] \\
& 0_{V}^{n}=\frac{i^{n-2}}{2 n !}\left[F^{\alpha \mu 1} D^{\mu 2} \cdots D^{\mu n-1} F_{a}^{\mu n}+\text { perm. }\right]
\end{aligned}
$$


Consider the electromagnetic current and a four flavor theory where

$$
J=\frac{2}{3} \bar{\phi}_{u} \phi_{u}-\frac{1}{3} \bar{\phi}_{d} \phi_{d}+\frac{2}{3} \bar{\phi}_{c} \phi_{c}-\frac{1}{3} \bar{\phi}_{s} \phi_{s} \text {. }
$$

The time-ordered product is reduced by usual contractions to (keeping only the field operators)

$$
\begin{aligned}
T(J(z) J(0)) & =\frac{4}{9} \bar{\phi}_{u}(z) \psi_{u}(z) \bar{\phi}_{u}(0) \phi_{u}(0)+\frac{1}{9}(u+d)+\frac{4}{9}(u+c)+\frac{1}{9}(u+s) \\
& =\frac{4}{9} \bar{\phi}_{u}(z) \phi_{u}(0)+\frac{1}{9} \bar{\phi}_{d}(z) \phi_{d}(0)+\frac{4}{9} \bar{\phi}_{c}(z) \phi_{c}(0)+\frac{1}{9} \bar{\phi}_{s}(z) \psi_{s}(0) \\
& =\frac{5}{18} 0_{0}+\frac{1}{6} 0_{3}+\frac{1}{18} 0_{8}-\frac{1}{18} 0_{15} \quad \text { (R5.85,R5.88) (A32) }
\end{aligned}
$$

where

$$
\begin{aligned}
& 0_{0} \equiv \bar{\psi}_{u} \phi_{u}+\bar{\phi}_{d} \phi_{d}+\bar{\phi}_{s} \phi_{s}+\bar{\psi}_{c} \phi_{c} \\
& 0_{3} \equiv \bar{\psi}_{u} \phi_{u}-\bar{\phi}_{d} \phi_{d} \\
& 0_{8} \equiv \bar{\psi}_{u} \phi_{u}+\bar{\phi}_{d} \phi_{d}-2 \bar{\psi}_{s} \phi_{s} \\
& 0_{15} \equiv \bar{\psi}_{u} \phi_{u}+\bar{\phi}_{d} \phi_{d}+\bar{\phi}_{s} \phi_{s}-3 \bar{\phi}_{c} \phi_{c}
\end{aligned}
$$

Clearly $\mathrm{O}_{0}$ is identified as the fermionic singlet (under SU(4)) operator $\mathrm{O}_{F}$ in Eq. A30, and $\mathrm{O}_{3,8,15}$ are the flavor carrying (nonsinglet) operators $\mathrm{O}_{\mathrm{NS}}$. Define the parton distributions

$$
\begin{aligned}
& \Sigma \equiv\left\langle p\left|0_{0}\right| p\right\rangle=u+\bar{u}+d+\bar{d}+s+\bar{s}+c+\bar{c}=u_{v}+d_{v}+6 \xi+2 \xi^{\prime} \\
& A_{3} \equiv\left\langle p\left|0_{3}\right| p\right\rangle=u+\bar{u}-d-\bar{d}=u_{v}-d_{v} \\
& A_{8} \equiv\left\langle p\left|0_{8}\right| p\right\rangle=u+\bar{u}+d+\bar{d}-2 s-2 \bar{s}=u_{v}+d_{v} \\
& A_{15} \equiv\left\langle p\left|0_{15}\right| p\right\rangle=u+\bar{u}+d+\bar{d}+s+\bar{s}-3 c-3 \bar{c}=u_{v}+d_{v}+6 \xi-6 \xi^{\prime}
\end{aligned}
$$

where $\xi \equiv s \sim \bar{s} \sim \bar{u} \sim \bar{d}$, and $\xi^{\prime} \equiv c \sim \bar{c}$. Since the $0_{F}$ (related to $\Sigma$ ) and $0_{V}$ (related to gluon distribution G) in Eq. A30 have identical quantum numbers, they mix under renormalization and we have to deal with a $2 \times 2$ 
matrix of anomalous dimension $\gamma$ 's (see Eq. A26 and the comment following it). The dimensions have been calculated using Feynman rules implied by the operators to be (Fig. 94)

$$
\begin{aligned}
& \gamma_{11}=\gamma_{F F}^{F}=\gamma_{N S}=\frac{a}{2 \pi} \frac{4}{3}\left[1-\frac{2}{n(n+1)}+4 \sum_{j=2}^{n} \frac{1}{j}\right] \\
& \gamma_{22}=\gamma_{V V}^{V}=\frac{a}{2 \pi}\left[3\left[\frac{1}{3}-\frac{4}{n(n-1)}-\frac{4}{(n+1)(n+2)}+4 \sum_{j=2}^{n} \frac{1}{j}\right]+\frac{2}{3} N_{f}\right] \\
& \gamma_{12}=\gamma_{F F}^{V}=-\frac{a}{2 \pi} \frac{2\left(n^{2}+n+2\right)}{n\left(n^{2}-1\right)} \frac{4}{3} \\
& \gamma_{21}=\gamma_{V V}^{F}=-\frac{a}{2 \pi} \frac{4\left(n^{2}+n+2\right)}{n(n+1)(n+2)} \frac{1}{2} N_{f} .
\end{aligned}
$$

However, the matrix has the eigenvalues

$$
\gamma_{*}=\frac{1}{2}\left[\gamma_{F F}^{F}+\gamma_{V V}^{V} * \sqrt{\left(\gamma_{V V}^{V}-\gamma_{F F}^{F}\right)^{2}+4 \gamma_{V V}^{F} \gamma_{F F}^{V}}\right]
$$

and can be diagonalized by the transformation

$$
\begin{array}{lll}
0_{-}=a_{n} 0_{F}+\beta_{n} 0_{V} & \text { or } & 0_{F}=0_{-}+0_{+} \\
0_{+}=\left(1-a_{n}\right) 0_{F}-\beta_{n} 0_{v} & & \beta_{n} 0_{v}=\left(1-a_{n}\right) 0_{-}-a_{n} 0_{+}
\end{array}
$$

where

$$
\boldsymbol{\alpha}_{\mathrm{n}} \equiv\left(\boldsymbol{\gamma}_{\mathrm{FF}}^{\mathrm{F}} \boldsymbol{\gamma}_{+}\right) /\left(\boldsymbol{\gamma}_{-}-\boldsymbol{\gamma}_{+}\right), \quad \quad \boldsymbol{\beta}_{\mathrm{n}} \equiv \boldsymbol{\gamma}_{\mathrm{VV}}^{\mathrm{F}} /\left(\boldsymbol{\gamma}_{-}-\boldsymbol{\gamma}_{+}\right)
$$

The corresponding Wilson coefficients $C_{+}, C_{-}$and $C_{N S}$ would each satisfy a RG equation as in Eq. A25, and thus the corresponding parton distributions would evolve separately. From Eq. A37 and A28 we have the evolution

$$
\begin{aligned}
\left\langle x \Sigma\left(Q^{2}\right)\right\rangle_{n}= & {\left[a_{n}\left\langle x\left[\left(Q_{0}^{2}\right)\right\rangle_{n}+\beta_{n}\left\langle x G\left(Q_{0}^{2}\right)\right\rangle_{n}\right] R^{-a}-\right.} \\
& +\left[\left(1-a_{n}\right)\left\langle x \Sigma\left(Q_{0}^{2}\right)\right\rangle_{n}-\beta_{n}\left\langle x G\left(Q_{0}^{2}\right)\right\rangle_{n}\right] R^{-a}+ \\
\left\langle x G\left(Q^{2}\right)\right\rangle_{n}= & {\left[\left(1-a_{n}\right)\left\langle x G\left(Q_{0}^{2}\right)\right\rangle_{n}+\left(a_{n}\left(1-a_{n}\right) / \beta_{n}\right)\left\langle x\left[\left(Q_{0}^{2}\right)\right\rangle_{n}\right] R^{-a}-\right.} \\
& +\left[a_{n}\left\langle x G\left(Q_{0}^{2}\right)\right\rangle_{n}-\left(a_{n}\left(1-a_{n}\right) / \beta_{n}\right)\left\langle x\left[\left(Q_{0}^{2}\right)\right\rangle_{n}\right] R^{-a}+\right. \\
\langle x A & \left.\left(Q^{2}\right)\right\rangle_{n}=\left\langle x A_{i=3,8,15}\left(Q_{0}^{2}\right)\right\rangle_{n} R^{-a} N S
\end{aligned}
$$


where

$$
a_{*, N S} \equiv \gamma_{*, N S} / 8 \pi a b, \quad R \equiv \alpha\left(Q_{0}^{2}\right) / \alpha\left(Q^{2}\right)=\ln \left(Q^{2} / \Lambda^{2}\right) / \ln \left(Q_{0}^{2} / \Lambda^{2}\right)
$$

and $b$ defined by Eq. A11. These equations for the moments represent the leading $\log$ (to all orders) theoretical prediction in closed form. This moment analysis has a drawback because experimentally the structure functions at very small and large $x$ are hard to measure. With the aid of today's computers, it is possible to work directly in the $x$ space, with the integro-differential equations we will describe in the following section.

\section{A.2.2 Altarelli-Parisi Equations}

Some years after the evolution of the structure functions was derived (1973) [60] using the renormalization group equations, Altarelli and Parisi (1977) [61] found a very intuitive way to obtain exactly the same results (the idea was first formulated by Lipatov (1974) [62] for the pseudoscalar and Abelian glue models). Their new derivation is a generalization of the Weizsacker-Williams equivalent photon approximation [63] in QED and based on the intuitive parton picture of Kogut and Susskind [64]: by increasing the power of the "microscopen from $Q^{2}$ to $Q^{2}+\Delta Q^{2}$, one can discern a virtually emitted parton (from another parton) which could not be resolved at $Q^{2}$. The new picture suggests that the parton distributions, derived in the previous section from deep inelastic scattering, are process independent.

Define the resolving power $t \equiv \ln Q^{2} / Q_{0}{ }^{2}$, and rewrite Eq. A13 as $a(0) / a(t)=1+4 \pi b a(0) t$,

with $b$ being defined in Eq. A11. The moments, $M_{n} \equiv \int_{0}^{1} d x x^{n-2} M(x)$ (see Eq. A21), of the flavor non-singlet (or the diagonalized) structure functions have the form (see Eq. A28, or Eq. A39-A41)

$$
M_{n}(t)=M_{n}(0)\left[\frac{a(0)}{a(t)}\right]^{-c / 2 b}=M_{n}(0)(1+4 \pi b a(0) t)^{-c / 2 b}
$$

with $c \equiv \gamma(g) / g^{2}$ for the anomalous dimension of the corresponding operator (see Eq. A8, A35 and A36). It satisfies the differential equation

$$
d M_{n}(t) / d t=-2 \pi c a(t) M_{n}(t)=(\alpha(t) / 2 \pi) M_{n}(t)\left(-4 \pi^{2} c\right)
$$


Define a function $P(z)$, such that

$$
\int_{0}^{1} d z z^{n-1} P(z) \equiv-4 \pi^{2} c=-\frac{\pi}{a} \gamma
$$

and rewrite Eq. A45 in terms of parton distributions

$$
\begin{aligned}
\frac{d}{d t} \int_{0}^{1} d x x^{n-1} q(x, t) & =\frac{a(t)}{2 \pi} \int_{0}^{1} d y y^{n-1} q(y, t) \int_{0}^{1} d z z^{n-1} P(z) \\
& =\frac{a(t)}{2 \pi} \int_{0}^{1} \int_{0}^{1} d y d z(y z)^{n-1} q(y, t) P(z) \\
& =\frac{a(t)}{2 \pi} \int_{0}^{1} d x x^{n-1} \int_{0}^{1} \int_{0}^{1} d y d z \delta(x-y z) q(y, t) P(z)
\end{aligned}
$$

Thus we have

$$
\begin{aligned}
\frac{d}{d t} q(x, t) & =\frac{a(t)}{2 \pi} \int_{0}^{1} \int_{0}^{1} d y d z \delta(x-y z) q(y, t) P(z) \\
& =\frac{a(t)}{2 \pi} \int_{x}^{1} \frac{d y}{y} q(y, t) P(x / y)
\end{aligned}
$$

This equation can be interpreted as follows: given a quark with momentum $y$ there is a chance $d t \cdot(a(t) / 2 \pi) \cdot P(z) d z$ that it radiates a gluon and reduces its energy from $y$ to $x=y z$. The parton density at $x$ simply receives all contributions from $y>x$.

For general situations including flavor singlet structure functions, we have the following master equations

$$
\begin{aligned}
& \frac{d q^{i}(x, t)}{d t}=\frac{a(t)}{2 \pi} \int_{x}^{1} \frac{d y}{y}\left[\sum_{j=1}^{2 N_{f}} q^{j}(y, t) P_{q i+q j}(x / y)+G(y, t) P_{q i+G}(x / y)\right] \\
& \frac{d G(x, t)}{d t}=\frac{a(t)}{2 \pi} \int_{x}^{1} \frac{d y}{y}\left[\sum_{j=1}^{2 N_{f}} q^{j}(y, t) P_{G+q j}(x / y)+G(y, t) P_{G+G}(x / y)\right]
\end{aligned}
$$

provided that

$$
\int_{0}^{1} d z z^{n-1}\left[\begin{array}{ll}
P_{q q}(z) & P_{G q}(z) \\
2 N_{f} P_{q G}(z) & P_{G G}(z)
\end{array}\right] \equiv-(\pi / a)\left[\begin{array}{cc}
\gamma_{F F}^{F} & \gamma_{F F}^{V} \\
F & V \\
\gamma_{V V} & \gamma_{V V}
\end{array}\right] .
$$

where the $\gamma$ 's are the anomalous dimensions given in Appendix A ((A35)) .

If $P_{i}(z)$ are merely defined through Eq. A50, there would be no "new derivation" of the structure function evolution because we just have another convenient (and useful because of today's computer) way of looking at the $O P E+R G$ results. The fact is that $P_{i}(z)$ indeed can be derived from 
QCD vertices. Since the emissions are virtual (though still not too far off-shell in order to be quasi-real particles - partons), the decay functions $P(z)$ 's are derived through the two cross sections $\mathrm{d} \sigma_{a}(A+D \rightarrow C+B+D \rightarrow C+F)$, and $d \sigma_{b}(B+D \rightarrow F)$. The cross sections are related through

$$
\mathrm{d} \sigma_{\mathrm{a}}=\mathrm{dP} \mathrm{BA}_{\mathrm{BA}}(\mathrm{z}) \mathrm{dz} \mathrm{d} \sigma_{\mathrm{b}} .
$$

With the approximation (define $k_{A}, k_{B}$, and $k_{C}$ to be the momenta of the particles)

$$
\begin{aligned}
& k_{A}=(P ; P, \delta), \\
& k_{B}=\left(z P+P_{t}^{2} / 2 z P ; z P, \vec{P}_{t}\right), \text { and } \\
& k_{C}=k_{B}\left(z \rightarrow 1-z, \vec{P}_{t} \rightarrow-\vec{P}_{t}\right),
\end{aligned}
$$

the decay functions have been derived to be

$$
\begin{aligned}
& P_{q q}(z)=\frac{4}{3} \frac{1+z^{2}}{(1-z)+2 \delta(1-z)} \\
& P_{q g}(z)=\left[z^{2}+(1-z)^{2}\right] / 2 \\
& P_{g q}(z)=\frac{4}{3} \frac{1+(1-z)^{2}}{z} \\
& P_{g g}(z)=6\left[\frac{z}{(1-z)_{+}}+\frac{1-z}{z}+z(1-z)+\left(\frac{11}{12}-\frac{N f}{18}\right) \delta(1-z)\right],
\end{aligned}
$$

with the distribution $1 /(1-z)$, being defined by

$$
\int_{0}^{1} d z \frac{f(z)}{(1-z)_{+}} \equiv \int_{0}^{1} d z \frac{f(z)-f(1)}{1-z} \text {, and }(1-z)_{+}=(1-z) \text { for } z<1 \text {, }
$$

for Bloch-Nordsieck cancellation of infrared divergences. The moments of these decay functions can be easily evaluated, and they are exactly those in Eq. A50. Thus the structure function evolution has been rederived by A-P in a "visibly" superior way. However, it requires an input function $\alpha(t)$ which must be determined elsewhere (field theory, Sect. A.1).

\section{A.2.3 Summation of Bethe-Salpeter Ladders}

The third road to Roma is: based on the fact that in the "axial gauge" $(n . A=0)$ only the ladder-like diagrams give rise to the leading $\ln Q^{2}$ terms (terms like $\left(a \ln Q^{2}\right)^{n}$, but not $a^{m}\left(a \ln Q^{2}\right)^{n}$ with $\left.m>0\right)$. Since this is a 
generally true fact, whether the virtual states are spacelike (prior to hard collision) or are timelike (after hard collision), this technique of ladder sumnation can be used in various QCD applications other than the structure functions. Instead of going into its formalism (Bethe-Salpeter equations) [65], let's only apply the parton splitting functions (which are the results of QCD vertices) of previous section to the ladders and show how it gives the same results as the previous methods did and provides an even more transparent picture.

Consider the ladder diagram in Fig. 95. In the region where $k$ can be given by $k=(1-y) p$ (colinear region which gives leading $l o g$ ), the phase space is approximated by

$$
\frac{d k^{3}}{2 k^{0}(2 \pi)^{3}}=\frac{d y d t_{i}}{16 \pi^{2}}
$$

where $t_{i} \equiv p_{i}^{2}$. The relation between the $i-1$ rung and $i$ rung cross sections is given by

$$
\sigma_{i}\left(p, q, p^{2}=m^{2}\right)=\int \frac{d y}{y} \frac{d t_{i}}{t_{i}} \frac{a}{2 \pi} P_{q q}(y) \sigma_{i-1}\left(y p, q,(y p)^{2}=t_{i}\right) .
$$

Moreover, it follows from kinematical considerations that the virtualities $t_{i}$ are ordered

$$
p^{2} \leq\left|t_{i}\right| \leq\left|t_{i-1}\right| \leq \cdots \leq\left|t_{1}\right| \leq Q^{2}
$$

The ladder must be dressed, i.e. include all virtual radiative corrections, and we should use the running coupling $a\left(t_{i}\right)=\left(4 \pi b \ln \left(t_{i} / \Lambda^{2}\right)\right)^{-1}$ at each vertex. The iteration of Eq. A56 for the whole ladder gives

$$
\begin{array}{r}
\sigma_{i}=\frac{1}{\left(8 \pi^{2} b\right)^{j}} \int_{m^{2}}^{Q^{2}} \frac{d t_{1}}{t_{1} \ln t_{1}} \int_{m}^{t_{1}} \frac{d t_{2}}{t_{2} \ln t_{2}} \cdots \int_{m}^{t_{i-1}} \frac{d t_{i}}{t_{i} \ln t_{i}} x \\
\int_{0}^{1} \frac{d y}{y} P_{q q}(y) \int_{0}^{y} \cdots \int_{0}^{r} \frac{d z}{z} P_{q q}(z / r) \delta(1-x / z)
\end{array}
$$

Taking the moments, we have

$$
\begin{aligned}
\int_{0}^{1} d x x^{n-1} \sigma_{i} & =\frac{1}{\left(8 \pi^{2} b\right)^{j}} \frac{1}{j !}\left(\ln \ln \frac{Q^{2}}{m^{2}}\right)^{j}\left[\int_{0}^{1} d y y^{n-1} P_{q q}(y)\right] \\
& =\frac{1}{j !}\left[-\frac{c(n)}{2 b} \ln \ln \frac{Q^{2}}{m^{2}}\right]^{j}
\end{aligned}
$$


When all ladders of different number of rungs are summed, i.e. summed over $j$, we get the desired exponents as in Eq. A44.

We remember that in A-P method, the quantity $\ln Q^{2} / Q_{0}^{2}$ was referred to as the resolving power of the "microscope". The above exercise gives us the picture that in leading log approximation (LLA) a parton, with changing virtuality $t_{i}$, makes incoherent radiations with the probability (rather than amplitude) at each vertex given by $a\left(\left|t_{i}\right|\right) / 2 \pi\left|t_{i}\right|$ times the A-P splitting functions. The virtuality $\left|t_{j}\right|$ increases (decreases) as the decaying parton approaches (moves away from) the hard collision. In the initial state before a hard collision the parton becomes more and more spacelike (its virtuality increases by approximately $-k_{t}{ }^{2}$ at each splitting) and the emitted partons must reassemble if no collision occurs. After collision the final state partons are in timelike virtual states and successively decay into low mass partons (which then hadronize). We will discuss the final state parton showering and initial state radiation in Appendix B.

\section{A.3 Parameterizations of the Structure Functions}

The quark (but not gluon) distributions can be measured through electroweak interactions by the relations [66]

$$
\begin{aligned}
& F_{2}^{e p}=\frac{4}{9} \times(u+\bar{u})+\frac{1}{9} \times(d+\bar{d})+\frac{1}{9} \times(s+\bar{s})+\cdots=F_{2}^{e n}(w i t h u+d) \\
& F_{2}^{\nu p}=2 \times(d+\bar{u})+\cdots=F_{2}^{\overline{\nu n}} \\
& F_{2}^{\nu n}=2 \times(u+\bar{d})+\cdots=F_{2}^{\overline{\nu p}} \\
& F_{3}^{\nu p}=2(\bar{u}-d)+\cdots=F_{3}^{\bar{\nu}} \\
& F_{3}^{\nu n}=2(\bar{d}-u)+\cdots=F_{3}^{\overline{\nu p}} .
\end{aligned}
$$

Once the quark distributions are determined, the shape of the $G(x)$ is constrained because of A-P equations. Intuitively, gluons with momentum 
fraction $x_{0}$ would generate antiquarks with momentum fraction $x_{1}>x_{0}$, and a failure to find antiquarks at values of $x$ larger than some $x_{0}$ thus constrains $G\left(x, Q_{0}{ }^{2}\right)$. Besides, the quantity $\int d x x G(x)$ is also determined from the momentum sum rule. There have been several sets of parameterizations proposed by several authors, all starting from low energy data and mostly parameterizing the evolved structure functions in terms of $Q^{2}$. In the following we will only mention two of the most popular ones (not necessarily the best): Duke-Owens' (DO) and Eichten-Hinchliffe-Lane-Quigg's (EHLQ).

\section{A.3.1 Duke-Owens (DO) [51]}

DO structure functions are based on several sets of data: deep inelastic neutrino, muon and electron scattering data (MIT-SLAC, CDHS and European Muon Collaboration (EMC)), dimuon mass distributions (Columbia-FermilabStonyBrook and CERN ISR) and $J / \Psi x_{F}$ distributions (Chicago-Princeton). The valence quark distributions were assumed to have the form

$$
\begin{aligned}
& x\left(u_{v}+d_{v}\right)=N_{u d} x^{\eta_{1}}(1-x)^{\eta_{2}}\left(1+\gamma_{u d} x\right), \\
& x d_{v}=N_{d} x^{\eta_{3}}(1-x) \eta_{4}\left(1+\gamma_{d} x\right) .
\end{aligned}
$$

The gluon, sea quark, and charm distributions $\times G, \times S(S \equiv 2(\bar{u}+\bar{d}+\bar{s}))$ and $\times c$ have the general form

$$
A x^{a}(1-x)^{b}\left(1+\alpha x+\beta x^{2}+\gamma x^{3}\right)
$$

All parameters in these parameterizations are assumed to have the form $A(s)$ $=A_{0}+A_{1} s+A_{2} s^{2}$, where $s=\ln \left[\ln \left(Q^{2} / \Lambda^{2}\right) / \ln \left(Q_{0}^{2} / \Lambda^{2}\right)\right]$. The fitting program operates by directly integrating the A-P equations including next-toleading order terms $\left(e . g . P_{q+}\right)$ [67]. Note that for any value of $x$ only distributions in the range between $x$ and 1 are required to do the integration, and thus the uncertainty in the low $x$ region can be avoided (also see the end of section A.2.1).

Because the gluon distribution can only be inferred from the sea quark distributions and the available data could not make distinction between soft gluon distribution with smaller $\Lambda$ and hard gluon distribution with larger $\Lambda$, Duke and Owens provide two sets of fits. In set $I$, they use the 
$\mathrm{J} / \mathrm{Psi}$ and dimuon data to constrain (directly or indirect/y) the gluon distribution. In set II, the dimuon and J/Psi data were not used and a broader gluon distribution was intentionally chosen (by choosing different $\eta_{G}$ ). However, there is an indication as pointed out by EHLQ [52] that the hard gluon parameterization (DO2) can not be trusted for $Q^{2}>10^{5} \mathrm{GeV}^{2}$.

\section{A.3.2 Eichten-Hincliffe-Lane-Quigg (EHLQ) [12]}

EHLQ was based exclusively on the CDHS data. Instead of parameterizing the evolved results in terms of $Q^{2}$ as other parameterizations do, the EHLQ method directly integrates the A-P equations (which have been recast as equations for $x$ times the parton distributions $(x f(x))$ to improve numerical convergence in the neighborhood of $x=0$ ). The $A-P$ equations are to the leading order only, and it was argued that either the higher order corrections are small (suppressed by one power of $a$ ), or the structure functions are small when corrections are big (for very large $x$ ). There are also two sets of starting parameterizations, which were modified from the two sets of parameterizations made by $\mathrm{CDHS}$ at $\mathrm{Q}_{0}^{2}=5 \mathrm{GeV}^{2}$. The first set was obtained (by CDHS) by assuming the hard-to-measure ratio $R=\sigma_{\mathrm{L}} / \sigma_{\mathrm{T}}=$ 0.1 when they used singlet structure functions and antiquark distributions to make the simultaneous fit to $\Lambda$ and $G(x)$. The second set was obtained by assuming that $R$ has the behavior prescribed by $Q C D$.

To aid people who may not be able to use their numerical results, they have also carefully parameterized the $Q^{2}$ dependence in terms of orthogonal Chebyshev polynomials (inspired by the $x \rightarrow 1$ behavior of structure functions). The $x$ range was divided at $x=0.1$ and each region has $i$ ts own parameterization. 


$$
\left.\mathrm{d} \sigma \sim \sum_{X}\right|_{N \rightarrow W^{\prime}} ^{1}
$$

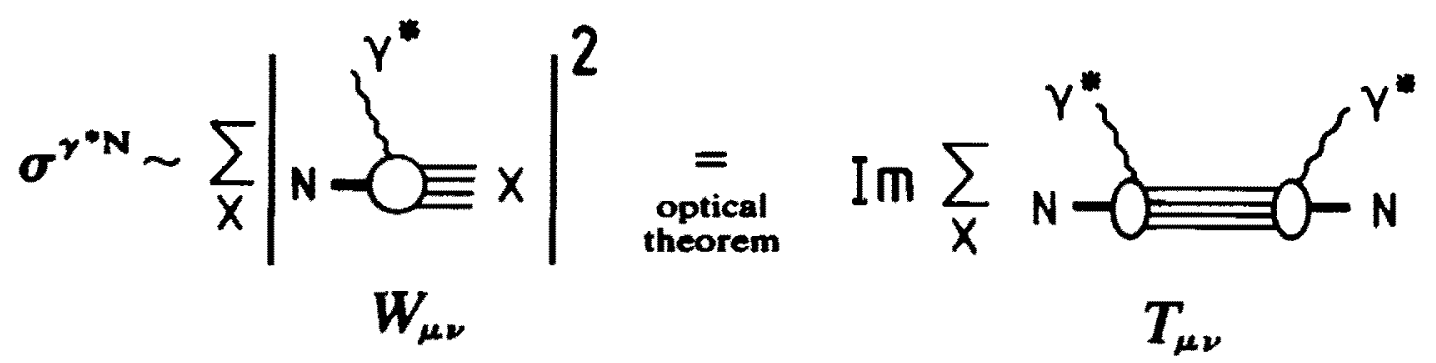

Fig. 92 The cross section for the deep inelastic scattering process $I+N$ $\rightarrow 1 \cdot+X . T_{\mu \nu}$ and $w_{\mu \nu}$ are defined in the text.
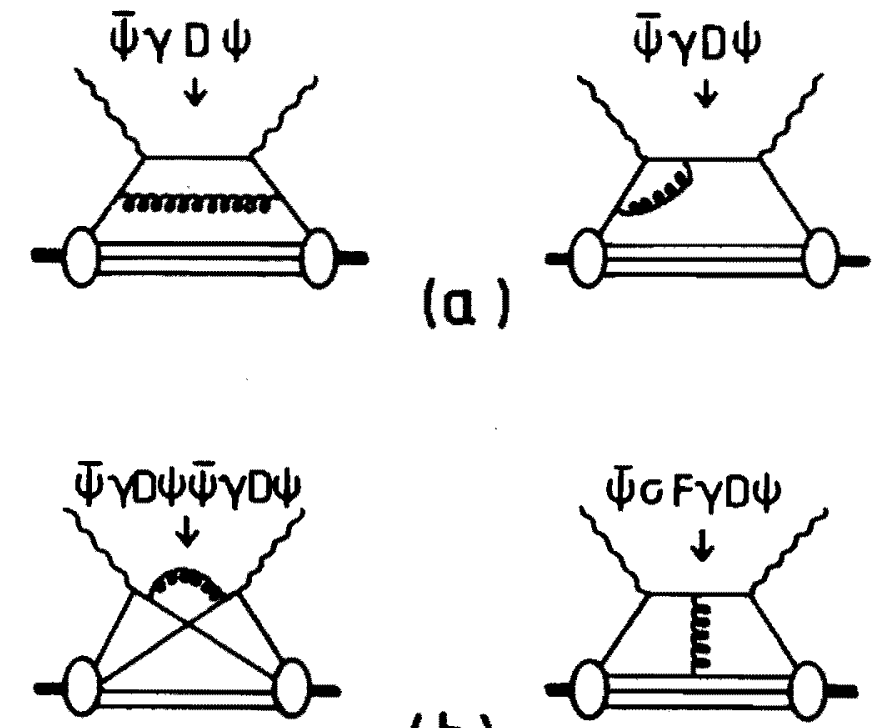

(b)

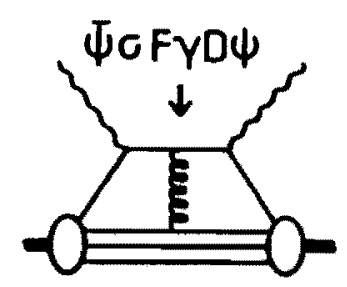

Fig. 93 Diagrams illustrating the meaning of twist: a) typical twist-2 effects, no communication between the struck quarks and spectator quarks, and b) twist-4 contributions. 


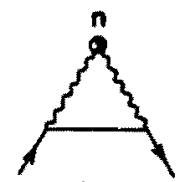

(a)

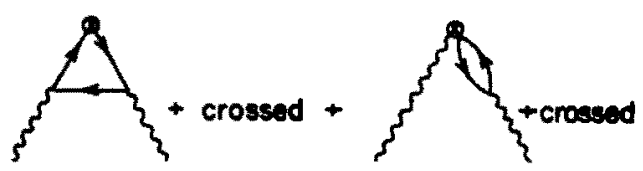

(b)
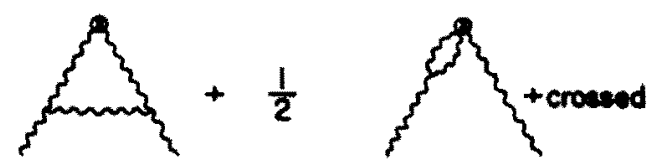

(c)
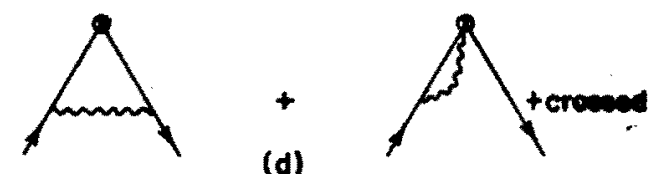

(d)

Fig. 94 Graphs for computing the matrix elements of: a) $D_{V}$ between fermion states $\left.\left(\gamma_{\mathrm{EF}}^{V}\right), b\right) 0_{F}$ between vector states $\left(\gamma_{\mathrm{YV}}^{F}\right)$, states $\left(\gamma_{V V}\right)$, and $\left.d\right) 0_{F}$ between fermion states $\left(0_{F F}^{F}\right)$.

c) $D_{V}$ between vector

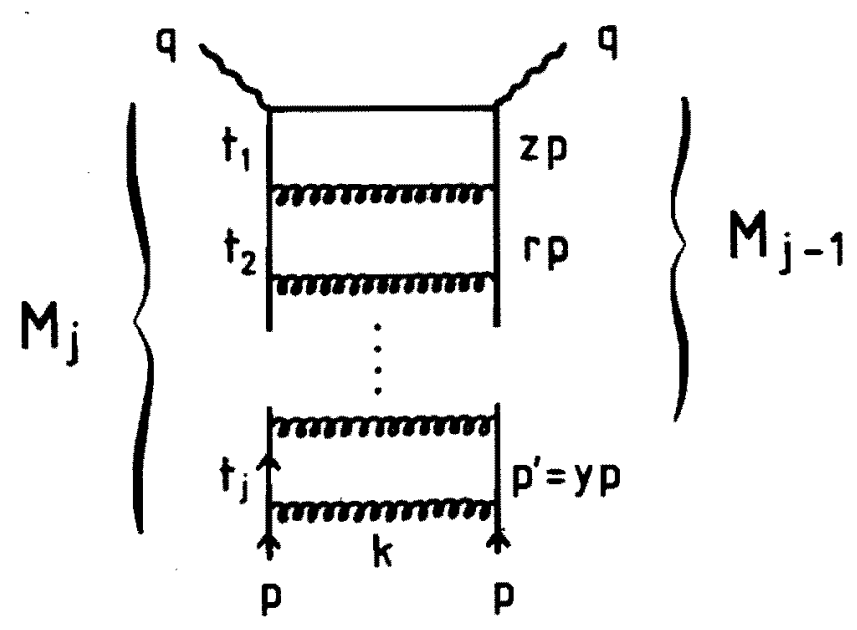

Fig. 95 Ladder diagram giving rise to leading mass singularities in nonsinglet structure functions. 


\section{APPENDIX B}

\section{HADRONIZATION OF QUARKS AND GLUONS}

Since no quarks have ever been found and QCD seems to indicate a phase of confinement, all observable objects are supposed to be color singlets. The transition from partons (quarks and gluons) to hadrons ("jets") is called hadronization or fragmentation. Three fragmentation schemes will be discussed in this appendix.

\section{B.1 Independent Fragmentation (I-F)}

This was proposed by Feynman and Field (F-F) [46] as the simplest way to do a complicated thing. Partons are treated as independent of each other. The fragmentation of a quark $q \rightarrow q^{\prime}+h$ is expressed by a primordial fragmentation function

$$
f(z)=1-a+a(b+1)(1-z)^{b} \text {, }
$$

where $z \equiv\left(E+P_{l}\right)_{h} /\left(E+P_{l}\right)_{q}$ is the longitudinal momentum fraction of the parton carried by the hadron. The values of $a$ and $b$ proposed by F-F are 0.88 and 2.0 respectively. A $Q^{2}$ dependent form of $f(z)$ has also been given by $A l i$ et al. [68]. Each hadron is then given a transverse momentum according to a gaussian distribution. The process is reiterated on the $q^{\prime}$ until the quark $E+P_{l}$ is below some cutoff. The gluon can be either fragmented like the quark with flavor randomly selected (with proper weights), or first broken into quark pairs which are then fragmented. In either case a different $f(z)$ may be used. The procedure inevitably leads to nonconservation of flavor, energy and momentum, and these are adjusted by hand afterward. Although the model is simple, it requires more parameters than any other models, e.g. the fragmentation function, the gaussian width of the hadron Pt distribution, the flavor ratio, and the ratio of scalar and vector particles, etc. 


\section{B.2 String Fragmentation (S-F)}

This is the scheme advocated by the LUND group [69]. As quarks $q$ and $\bar{q}$ move apart, a string (color field) is stretched between them (the stored energy is about $1 \mathrm{GeV} / \mathrm{fm}$; an $1 \mathrm{~m}$ long string would weigh 16 tons). The string may break by the production of a new quark pair q' $\bar{q}$ ' (at one point) with the quantum tunneling probability $\exp \left(-\pi \mathrm{m}_{t}^{2} / x\right)=\exp \left(-\pi \mathrm{m}^{2} / x\right)$ $\exp \left(-\pi p_{t}^{2} / \pi\right)$, where $m$ and $p_{t}$ are the quark mass and transverse momentum relative to the string, and $x$ is the string constant. Thus two color singlet systems $q \bar{q}$ ' and $q$ ' $\bar{q}$ are formed, one takes a fraction $z$ of the $W_{+} \equiv$ $E+P_{l}$ and $W_{+} W_{-} \equiv E^{2}-p_{l}^{2}=m_{t}{ }^{2}$ of the $q \bar{q}$ system and the other takes $1-z$. Each new string can break in a similar way if its mass is large enough, and at the end only ordinary hadrons remain. The gluon is treated as an energy and momentum carrying kink on a string (closed or open).

The various breaks have a spacelike separation, and thus the time ordering of breaking vertices is Lorentz frame dependent and hence irrelevant. The Monte Carlo procedure can start from either the quark end or the antiquark end of the string. The breaking point is chosen through a function $f(z)$, which is equivalent to the primordial fragmentation function used in I-F. In order to have symmetrical results for different starting ends of the string, LUND chose

$$
f(z)=z^{-1}(1-z)^{a} \exp \left(-b m_{t}^{2} / z\right) \text {. }
$$

\section{B.3 Parton Showering + Independent or Cluster Fragmentation}

As we stated at the end of Sect. A.2.3, in the leading log approximation, a parton makes incoherent radiation with the probability (rather than amplitude) at each vertex given by $a\left(t_{i}\right) / 2 \pi t_{i}$ times the A-P splitting functions. The virtuality $\left(\left|t_{i}\right| \equiv\left|p_{i}^{2}\right|\right)$ of the decaying parton increases as it approaches the hard collision, and decreases as it moves away. This picture is, strictly speaking, correct for small angle emissions only where colinear singularities give rise to leading logarithms. The branching approximation of Fox and Wolfram [70] is to also apply this picture to wide angle emission with the momentum fraction $z$ defined as the 
ratio of the $E+P_{l}$ of the emitted parton to that of the parent parton. This scenario is commonly called parton showering, and it has the effect of generating multijet configurations in a simple (but probably too simple) way.

When the parton showering is included in the final state, one can hadronize each final parton by the independent fragmentation scheme, as in the case in ISAJET [13] and COJETS/WIZJET [71] (but then the virtuality cutoff for parton branching needs to be higher, $6 \mathrm{GeV}$ in ISAJET as compared to 1-2 GeV in other Monte Carlo's, to insure that the number of final hadrons will not be unacceptably high). A totally new mechanism of fragmentation has been proposed (originally by Field and Wolfram [72]): First an ensemble of color-singlet quark-antiquark pairs (clusters) is formed from the partons, and then the clusters are decayed isotropically into 2 hadrons according to phase space (flavor, spin and kinematic). If a cluster is very massive (e.g. > $3.5 \mathrm{GeV})$, it is first broken into smaller ones using information on the color field (string) direction. The scheme is illustrated in Fig. 96 [73]. This is the model with the fewest parameters, as the particle ratios and $z$ and Pt distributions are all accounted for by phase space.

The interference between partons, so far ignored, has been shown to be not negligible in the soft ("infrared") region. But the effect is to cancel completely (in leading log approximation) the branching probability for certain regions of phase space and the surviving region can be described in simple terms: it is the one in which successive opening angles are ordered (uniformly decreasing) [74]. Imposing the angular ordering, in addition to the usual virtuality ordering, Webber [73] has his model of coherent cluster fragmentation. However, the experimental confirmation of this coherence is not trivial. 


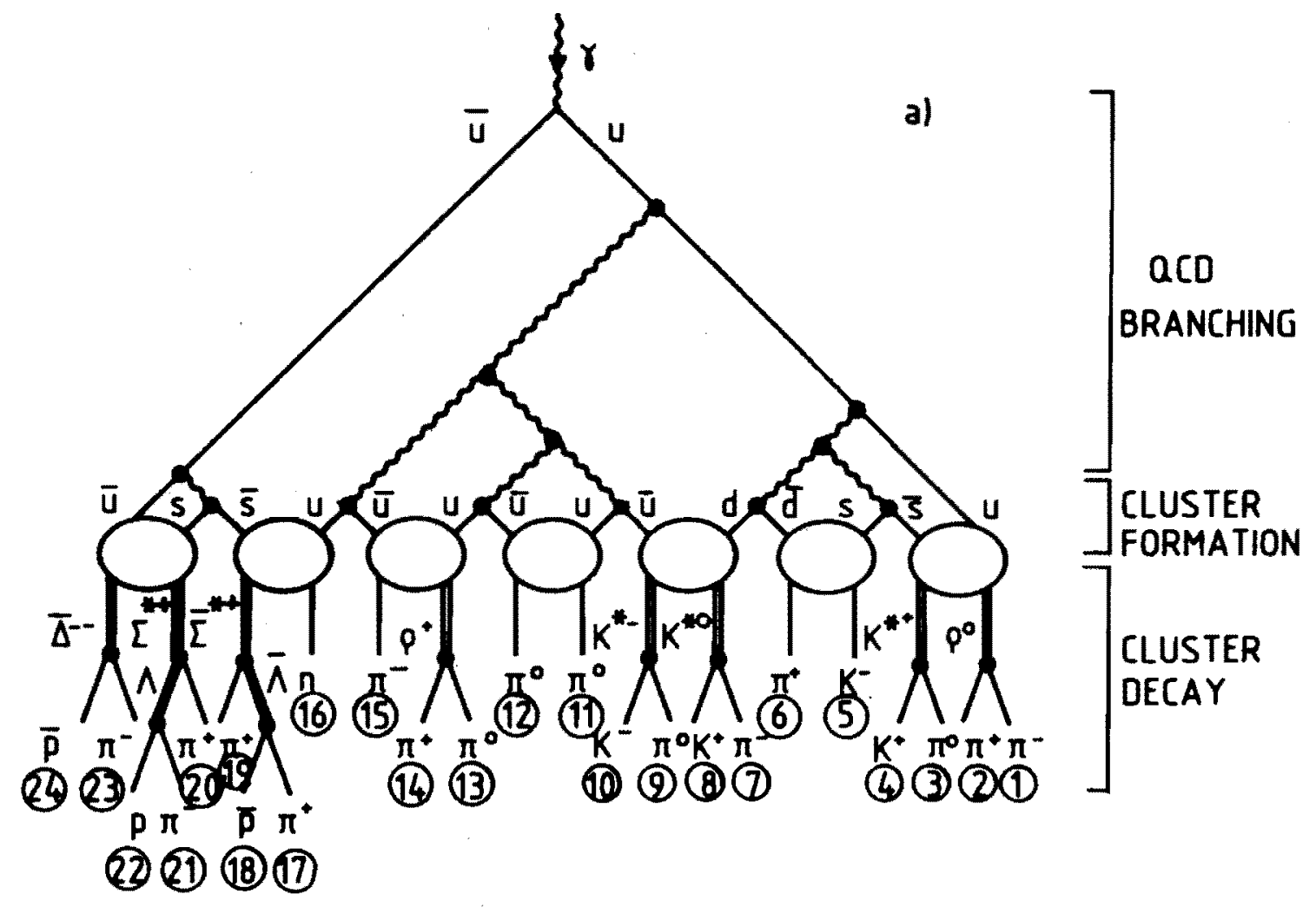

Fig. 96 Cluster fragmentation (from ref. 73). 


\section{APPENDIX C}

\section{BEAM JETS AND INITIAL STATE RADIATION}

The remaining cold parts of the baryons, after each being stripped off one parton, must also suffer softer impacts and find their ways to form final hadrons. We might think this system resembles that of minimum bias events (no hard parton collision involved) somehow, but certainly there are differences. First each remnant is not colorless (being stripped of one parton). Secondly, initial state radiation may be associated with the hard collision. Experiments at the SPPS have shown that in jet or $W-Z$ events the underlying energy density is about 1.5 - 2 times that of minimum bias events [47]. Furthermore the transverse kick of $W$ events was also found (7-8 GeV) to be much larger than Fermi motion would give $(<1 \mathrm{GeV})$. The prospect of a working theory for this soft part of hadron collisions might have improved over the years with the various ideas of fragmentation schemes, but the final theory is still not understood. Although QCD has greatly advanced the understanding of physics in the asymptotic region $\left(Q^{2}\right.$ $\rightarrow \infty)$, little can be said about soft processes. Coinciding with progress in the accelerator technology, QCD has shifted both theoretical and experimental interests toward very high energy collisions. The issues faced in 1960's regarding the production of hadrons continue to be unanswered. Presently, data on charged particle distributions (multiplicity, $\eta$, Pt and backward-forward correlation etc.) are being collected whenever a new center of mass energy is available, with the hope that some hints will be given on how to do this physics.

There have been two approaches to initial state radiations. One [75] is to start with a parton given by $Q^{2}$ independent structure functions, and evolve it to the point of making the hard collision. Both scaling violations and initial state radiation are generated in this way. But it has been found to be very inefficient for a Monte Carlo unless some thing sophisticated is done. Another [76] starts with scaling violating structure functions (the parameterizations of EHLQ, DO etc.), and evolves the 
colliding partons backward. The probability for a parton ' $b$ ' to disappear in the backward evolution is given by

$$
\begin{aligned}
d P_{b} & =\frac{1}{f_{b}(x, t)} \frac{d f_{b}(x, t)}{d t} d t \\
& =\frac{a(t)}{2 \pi t} d t \int_{a} \int_{x}^{1} \frac{d x^{\prime}}{x^{\prime}} \frac{f_{a}\left(x^{\prime}, t\right)}{f_{b}(x, t)} P_{a+b c}\left(x / x^{\prime}\right)
\end{aligned}
$$

The last step follows from the A-P equations. Even if we assume the initial state radiation is properly treated in LLA, there remains question on how to fragment these soft partons (ISAJET uses I-F and PYTHIA uses S-F, for example).

There are also several ways (as usual) [77] proposed to deal with the beam jets, but not all agree with data. One might treat the diquark remnant as another quark jet and fragment it by the independent fragmentation scheme (possibly also including by-hand a pedestal of additional activity). One might also treat them as another string system and do it in string fragmentation, or use longitudinal phase space parameterizations with multiplicity distributions and pedestal effects put in by hand. In PYTHIA, semihard interactions are generated according to Poisson distributions (with means depending on impact parameter). Ordinary strings are stretched out to the remnants. In ISAJET, a variable number of cut Pomerons (exchanged phenomenological "particles" with quantum numbers of the vacuum $I=S=B=0$ - the so called vacuum trajectory responsible for diffractive forward scattering which is related to particle production through the optical theorem) are used, each corresponds to a pair of backto-back low Pt jets. The probabilities $P_{N}$ for $N$ cut Pomerons are taken to be energy independent and are adjusted to fit the experimental data. The fragmentation parameters are made energy dependent by hand, $b(s)=b_{0}$ + $b_{1} \ln (s)$, to incorporate the observed increase in $d n / d y$. 


\section{APPENDIX D}

WHY ARE JETS ROUND IN $\eta-\phi$ SPACE?

The longitudinal (in Z-direction) rapidity, or simply called rapidity, of a particle is defined as

$$
y \equiv 1 / 2 \ln ((E+P z) /(E-P z)) \text {. }
$$

It follows then that under a longitudinal Lorentz boost y transforms as

$$
y \rightarrow y+(1 / 2) \ln ((1+\beta) /(1-\beta))
$$

and $d y=d P / E$ is an invariant. The rapidity is particularly useful in studying hadrons because the system of the colliding partons (or parton plus lepton) is always boosted with variable amount relative to the lab frame.

If particle mass is zero or negligible compared to particle energy, $y$ depends only on the polar angle

$$
y=-\ln (\tan (\theta / 2)) \text {. }
$$

This purely geometrical, $\theta$-dependent quantity has been widely used (called pseudo-rapidity and denoted as $\eta$ ) by experimenters in designing detector and data analysis despite the fact that in real world most particles are massive. When we come to the physics of jets, there is yet another surprise to be found that jets are approximately round in the $\eta-\phi$ space. To see this, it suffices to consider jets in the rest frame of the 2 parton system. Any longitudinal boost would leave the jet widths both in $\eta-$ direction and in $\phi$-direction invariant for the reason explained above (for $\eta$ ) and that particle $P_{x}$ and $P y$ are invariant under the boost. In the $C M$ frame, each parton. fragments into hadrons which are on average symmetrically distributed around the direction of the parton momentum. Let's draw a cone with opening angle $\Delta$ to represent a jet, and tilt it at any polar angle $\theta$ to intersect with a cylinder of radius $R$. Then we have

$$
\begin{aligned}
& d \eta=(d(-\ln (\tan (\theta / 2))) / d \theta) \Delta=(-1 / \sin \theta) \Delta \text { and } \\
& d \phi=(R / \sin \theta) \Delta(1 / R)=(1 / \sin \theta) \Delta,
\end{aligned}
$$

which show the roundness of jets. At first glance, it seems that a jet in the small angle region is bigger due to the factor $1 / \sin \theta$. We can show this 
is not the case for jets with the same $\mathrm{Pt}$ (momentum $\mathrm{P}=\mathrm{Pt} / \mathrm{sin} \theta$ ). If the particle's transverse momentum distribution relative to the jet axis does not depend on $P$, then the jet size $(\Delta)$ would be proportional to $1 / P=\sin \theta / P t$ and we have $d \eta=d \phi=C(1 / \sin \theta)(\sin \theta / P t)=C / P t$, which is independent of polar angle.

Thus for a given $P t$, jets are round in $\eta-\phi$ space and have the same size everywhere. 


\section{APPENDIX E}

\section{THE CDF SHOWER SIMULATION}

Since showering is the principal process on which the calorimetry measurement is based on, we will give the shower parameterizations used in the CDF simulation [78]. At the birth of each particle, the number of interaction (or radiation) lengths it will travel is ordained. As the particle is stepped through the detector, the number of interaction lengths traversed is incremented, and if the particle has lived long enough it showers.

\section{E.1 Longitudinal Development of EM Shower}

$$
\begin{aligned}
& d E / d Z=K S^{a-1} \exp (-b S) \\
& a=2.1+0.56 \ln (E)+\Delta a \\
& b=0.515+\Delta b
\end{aligned}
$$

where

$K$ : a normalization such that the integral equals the energy of the showering particle;

$S$ : the number of radiation lengths traversed in $Z \mathrm{~cm}$ since shower starts;

$a$ and $b$ : fit values;

$\Delta a$ and $\Delta b$ : randomly extracted from Gaussian distributions whose sigmas are 0.5 and 0.051 respectively. A positive correlation between a and $b\left(C_{a b}=0.83\right)$ is produced.

\section{E.2 Longitudinal Development of Hadronic Shower}

$$
\begin{aligned}
& d E / d Z=K\left[W S^{a-1} \exp (-b S)+(1-W) T^{c-1} \exp (-d T)\right] \\
& a=2.0+0.036 \mathrm{E} \quad \text { for } E<10 \mathrm{GeV} \\
& =1.62+0.31 \ln (E) \quad \text { otherwise } \\
& b=0.22
\end{aligned}
$$




$$
\begin{aligned}
& c=a \\
& d=1.01-0.024 \ln (E)
\end{aligned}
$$

where

K: a normalization such that the integral equals the energy of the showering particle;

$S$ : the number of radiation lengths traversed in $Z \mathrm{~cm}$ since shower starts;

$T$ : the number of interaction lengths traversed in $Z \mathrm{~cm}$ since shower starts;

W: the fraction of electromagnetic energy (so 1-W the fraction of hadronic energy) in the hadron induced shower. W has a uniform probability between 0.01 and 0.4 , then falls off as a gaussian with a mean of 0.4 and a sigma of 0.25 ;

$a, b, c$ and $d$ : fit values.

The longitudinal profile is fluctuated by a random multiplication factor, SCALE. 1/SCALE is a truncated Gaussian (mean 0.6 and sigma 0.45 ) between 0.01 and infinity. When SCALE is calculated, it is forced to be in the range 0.2 to 1.5 .

\section{E.3 Transverse Profile of EM Shower (2-Component Gaussian)}

$$
\begin{aligned}
& d E / d r=K\left[A \exp \left(-r^{2} / 2 \sigma_{1}^{2}\right)+(1-A) \exp \left(-r^{2} / 2 \sigma_{2}^{2}\right)\right] \\
& A=0.6 \\
& \sigma_{1}=\left(A_{1}+B_{1} S\right)\left(X_{\text {eff }} / \epsilon\right) \\
& \sigma_{2}=\left(A_{2}+B_{2} S\right)\left(X_{\text {eff }} / \epsilon\right) \\
& A_{1}=0.0, \quad B_{1}=0.389 \\
& A_{2}=8.19, \quad B_{2}=0.0
\end{aligned}
$$

where

K: a normalization factor;

A: the splitting of energy between a narrow and a wide component of the the shower profile;

$\sigma$ 's: component widths of the profile, linearly dependent on the total number of radiation lengths traversed by the shower;

$S$ : the total number of radiation lengths the shower has traversed; 
$X_{\text {eff }}$ : the effective radiation length of the current material;

$\epsilon$ : the critical energy of the material;

$B_{1}$ has Gaussian smearing around its mean value of 0.389 with a sigma of 0.055 .

\section{E.4 Transverse Profile of Hadronic Shower}

The EM component has the same transverse profile as the narrow component $\left(\sigma_{1}\right.$ above) of the EM shower profile. The hadronic component has a single Gaussian profile with sigma linearly growing with the number of $\mathrm{grams} / \mathrm{cm}^{2}$ traversed:

$$
\begin{aligned}
& \sigma_{3}=A_{3}+B_{3} \cdot\left(\text { grams } / \mathrm{cm}^{2}\right) \\
& A_{3}=6.45, B_{3}=0.07 .
\end{aligned}
$$

$B_{3}$ has a Gaussian fluctuation with sigma 0.02 about its mean value of 0.07 .

\section{E.5 The Calorimeter Response}

It is well known that hadronic showers give less pulse height than electromagnetic showers due to nuclear binding energy, neutrinos, particle masses, etc. The CDF calorimeters have been calibrated in a way such that the EM calorimeters give a mean pulse height of $50 \mathrm{GeV}$ for $50 \mathrm{GeV}$ incident electrons, and Hadron calorimeters give $50 \mathrm{GeV}$ for $50 \mathrm{GeV}$ charged pions which are minimum-ionizing in the $E M$ calorimeter. The following table gives the calorimeter response ratios for the EM energy $(W)$ and hadronic energy (1-W) in the simulation that has been fit to the test beam data.

Calorimeter

EM component (W)

Hadronic component (1-W)

EM

$$
1.0
$$

0.40

Hadron

1.15

0.90

The showers developed according to the above parameterizations and response ratios are geometrically shared by towers (or pads in the gas calorimeters), and the effective number of minimum ionization particles are calculated and fluctuated with a sigma of $\sqrt{\mathrm{N}}$. 
Finally, the procedure of test beam calibration was repeated for the simulation. $50 \mathrm{GeV}$ electrons and pions are sent into the tower centers and gains were adjusted to give the incident energy.

The nonlinear response as observed in the 1987 minimum bias data [45] is reproduced in the simulation [44] (see Fig. 97) by scaling the energy of the showering particles before the showers are developed. The above parameterizations do not generate the observed nonlinearity properly. 
Average Single-Track Ecal/P vs. P

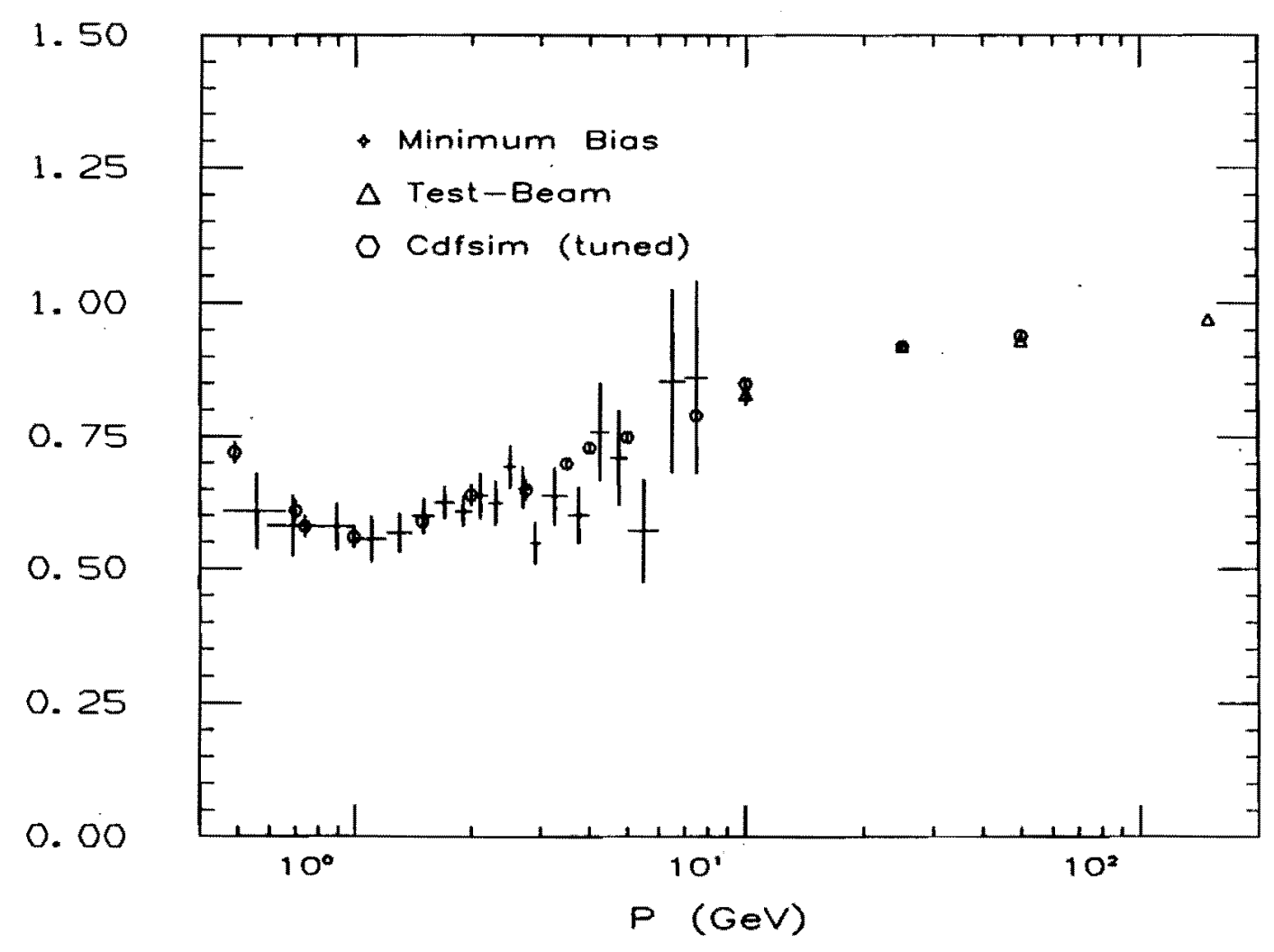

Fig. 97 The calorimeter response as a function of the charged particle momentum (from CDF684). 


\section{APPENDIX F}

\section{CHARACTERISTICS OF RUNS IN THE 1987 DATA}

Some of the characteristics of each run in the 1987 data sample are listed in the following table (Table 20). The quantities marked by **'s are somehow different from the average behavior of the other runs. Runs marked by *'s before their run numbers are excluded for use in our jet analysis. Most of them are due to high contamination of Main Ring splashes. Runs before 7214 are not used because the calibration constants were not downloaded into the hardware during the earlier period of run.

Definition of a "central cluster" : a cluster

a) with uncorrected Pt > $20 \mathrm{GeV}$ or corrected Pt > $30 \mathrm{GeV}$, and

b) with $|\eta|<1.0$ or physically landed on the central or endwall calorimeters.

Definitions of the various quantities in the table:

RUNNUM: the run number (sub-runs $A, B, C \ldots$ implied)

(Et) : hardware trigger total Et threshold, blank if it is $30 \mathrm{GeV}$

NEV: total number of events that passed the total Et trigger (on tape)

NMR: number of Main Ring events

NMRJ: number of Main Ring events, containing central clusters with Et above trigger Et threshold $(20,30,40$, or $45 \mathrm{GeV})$

NCR: number of central cosmic rays with Et above trigger Et threshold NJET: number of central jets with Et above trigger Et threshold ZMEAN: mean of event vertex $Z$ 's for events containing central jets with Et above trigger Et threshold ZWIDTH: width of the vertex $Z$ distribution

INTLUM: integrated luminosity $\left(n b^{-1}\right)$

XSEC: cross section $=$ NJET/INTLUM $(n b)$ 
Luminosity Information $\left(n b^{-1}\right)$ :

before 7214 (not shown in the table): 3.405

lost due to tape error $(7791 F G, 7802,7804): 0.856$

runs excluded (marked by '*' before RUNNUM) : 0.860

$20 \mathrm{GeV}$ runs (those not excluded) : 0.418

$30 \mathrm{GeV}$ runs (those not excluded) : 13.224

$40 \mathrm{GeV}$ runs (those not excluded): 6.139

$45 \mathrm{GeV}$ runs (those not excluded): 6.466

total $31.37\left(\left(n b^{-1}\right)\right.$ 
Table 20 Characteristics of runs in the 1987 data.

\begin{tabular}{|c|c|c|c|c|c|c|c|c|c|c|c|}
\hline 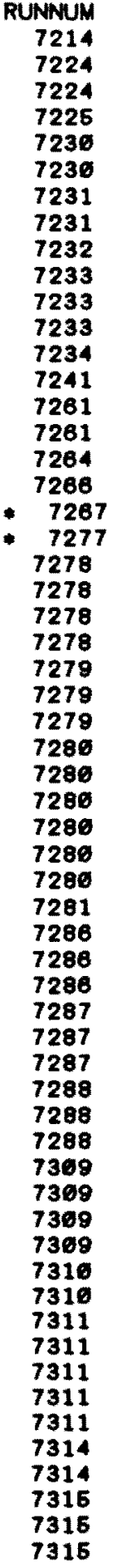 & 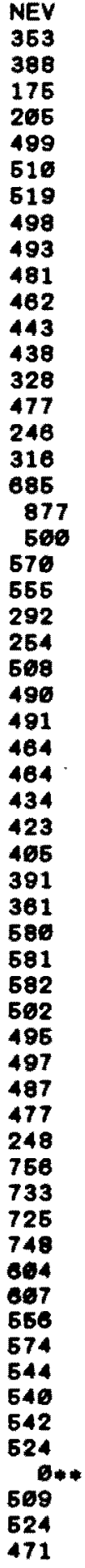 & 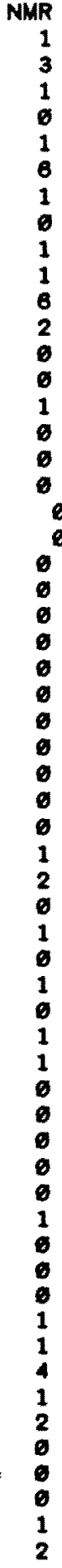 & 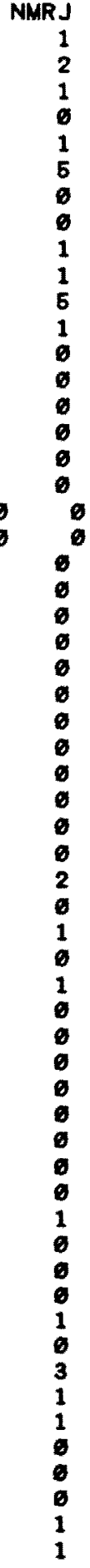 & 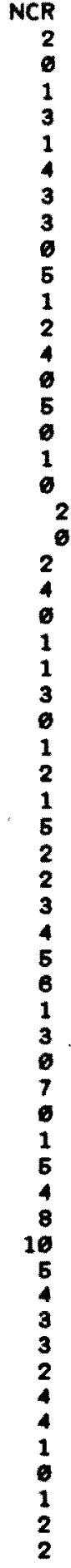 & $\begin{array}{r}\text { NJET } \\
168 \\
103 \\
75 \\
93 \\
191 \\
212 \\
245 \\
204 \\
212 \\
198 \\
198 \\
177 \\
178 \\
147 \\
215 \\
113 \\
136 \\
112 \\
75 \\
32 \\
261 \\
228 \\
133 \\
182 \\
212 \\
260 \\
214 \\
189 \\
263 \\
187 \\
170 \\
189 \\
169 \\
162 \\
232 \\
244 \\
265 \\
220 \\
214 \\
220 \\
216 \\
264 \\
110 \\
294 \\
294 \\
314 \\
313 \\
281 \\
276 \\
232 \\
251 \\
236 \\
237 \\
265 \\
246 \\
224 \\
209\end{array}$ & 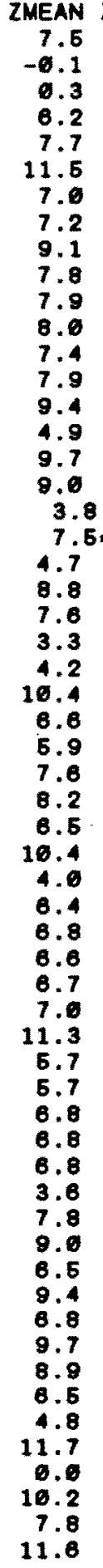 & $\begin{array}{r}\text { ZWIDTH } \\
27.5 \\
35.3 \\
33.7 \\
39.9 \\
36.2 \\
36.1 \\
35.2 \\
33.6 \\
33.1 \\
36.6 \\
34.5 \\
38.2 \\
42.9 \\
37.8 \\
29.2 \\
31.8 \\
31.2 \\
35.1 \\
36.9 \\
26.0 \\
32.4 \\
32.1 \\
34.3 \\
39.1 \\
36.3 \\
38.5 \\
34.1 \\
35.8 \\
36.5 \\
46.2 \\
39.6 \\
40.9 \\
37.7 \\
37.5 \\
35.2 \\
31.8 \\
31.3 \\
32.4 \\
35.6 \\
33.4 \\
37.4 \\
35.7 \\
41.5 \\
31.2 \\
30.0 \\
33.3 \\
29.0 \\
36.7 \\
36.3 \\
36.9 \\
38.2 \\
37.0 \\
37.8 \\
37.9 \\
41.8 \\
0.0 \\
39.4 \\
42.6 \\
39.7\end{array}$ & 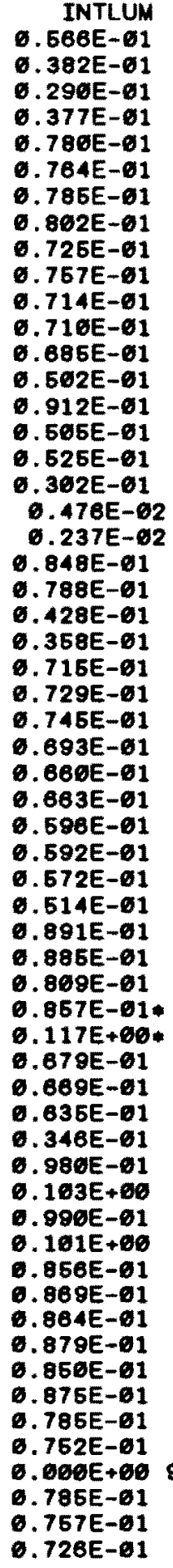 & 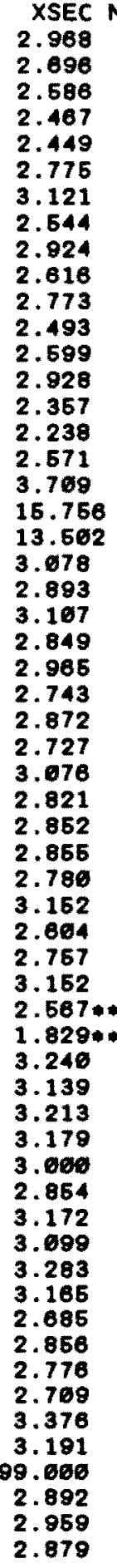 & 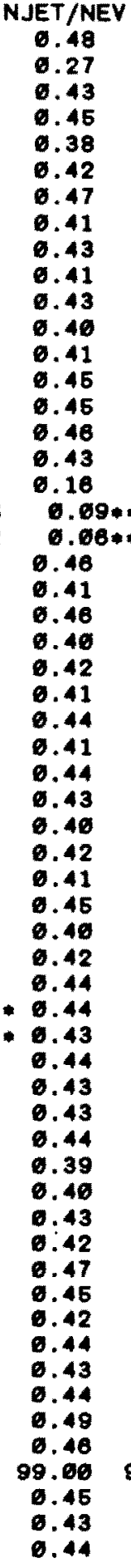 & $\begin{array}{l}\text { NMRJ/NJET } \\
0.01 \\
0.02 \\
0.01 \\
0.00 \\
0.01 \\
0.02 \\
0.00 \\
0.00 \\
0.00 \\
0.01 \\
0.03 \\
0.01 \\
0.00 \\
0.00 \\
0.00 \\
0.00 \\
0.00 \\
0.00 \\
0.00 \\
0.00 \\
0.00 \\
0.00 \\
0.00 \\
0.00 \\
0.00 \\
0.00 \\
0.00 \\
0.00 \\
0.00 \\
0.00 \\
0.00 \\
0.00 \\
0.01 \\
0.00 \\
0.00 \\
0.00 \\
0.00 \\
0.00 \\
0.00 \\
0.00 \\
0.00 \\
0.00 \\
0.00 \\
0.00 \\
0.00 \\
0.00 \\
0.00 \\
0.00 \\
0.00 \\
0.00 \\
0.00 \\
0.01 \\
0.00 \\
0.00 \\
0.00 \\
0.00 \\
0.00 \\
0.00 \\
\end{array}$ \\
\hline
\end{tabular}


Table 20 Characteristics of runs in the 1987 data (continued).

\begin{tabular}{|c|c|c|c|c|c|c|c|c|c|c|c|}
\hline 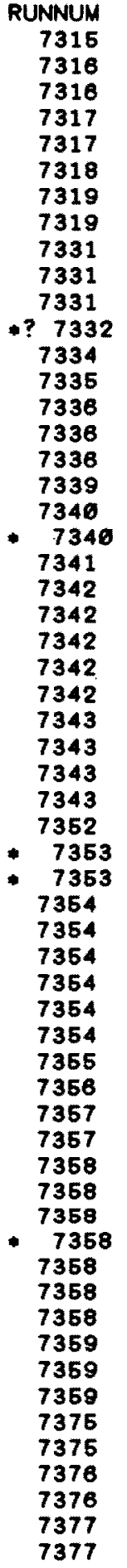 & 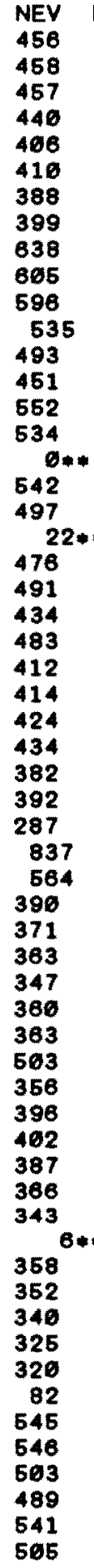 & 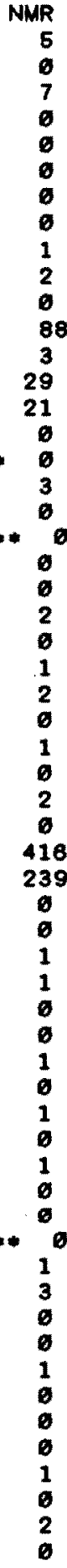 & 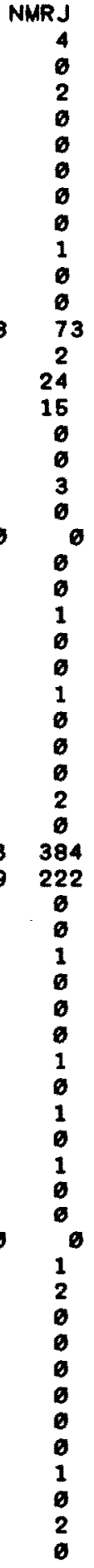 & 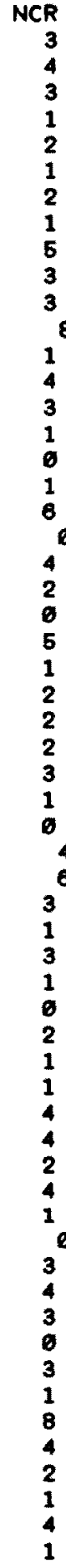 & 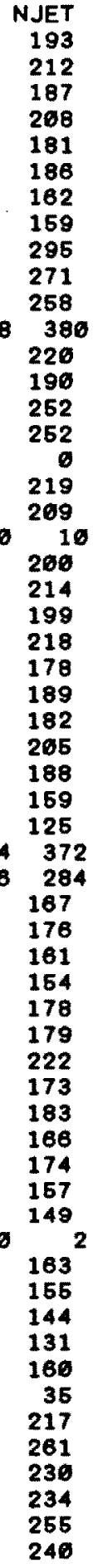 & 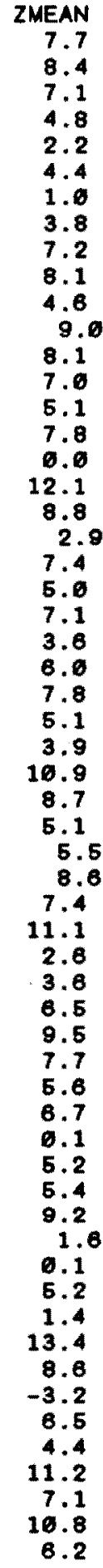 & $\begin{array}{r}\text { ZWIDTH } \\
37.6 \\
39.6 \\
39.1 \\
41.7 \\
41.3 \\
46.7 \\
46.6 \\
46.4 \\
32.6 \\
35.6 \\
34.6 \\
36.4 \\
32.7 \\
39.5 \\
36.7 \\
37.5 \\
6.6 \\
34.6 \\
38.6 \\
37.4 \\
39.9 \\
35.2 \\
46.1 \\
38.1 \\
36.1 \\
41.6 \\
42.8 \\
39.3 \\
41.4 \\
38.2 \\
27.5 \\
31.8 \\
32.7 \\
35.6 \\
35.3 \\
39.2 \\
33.6 \\
35.7 \\
35.2 \\
36.8 \\
37.3 \\
39.3 \\
35.6 \\
43.6 \\
37.4 \\
46.6 \\
55.4 \\
39.8 \\
39.4 \\
41.9 \\
39.7 \\
42.4 \\
52.9 \\
30.3 \\
30.7 \\
32.1 \\
32.4 \\
36.1 \\
34.2\end{array}$ & 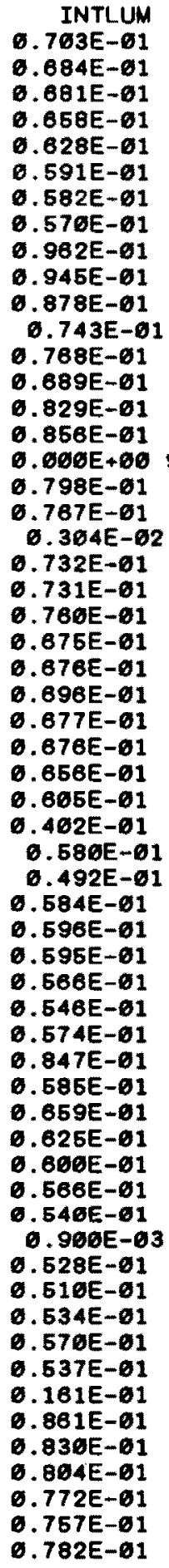 & 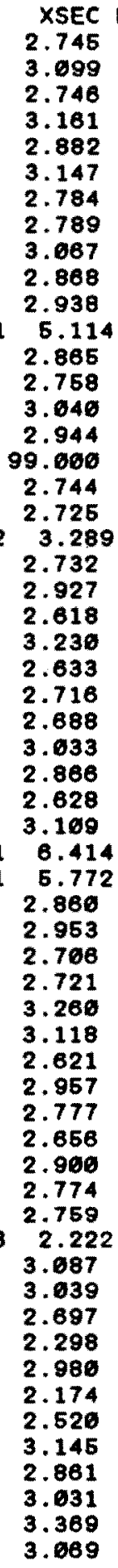 & $\begin{array}{l}\text { NJET/NEV } \\
0.42 \\
0.46 \\
0.41 \\
0.47 \\
0.45 \\
0.45 \\
0.42 \\
0.46 \\
0.46 \\
0.46 \\
0.43 \\
0.71 \\
0.45 \\
0.42 \\
0.46 \\
0.47 \\
99.00 \\
0.40 \\
0.42 \\
0.46 \\
0.42 \\
0.44 \\
0.46 \\
0.45 \\
0.43 \\
0.46 \\
0.43 \\
0.47 \\
0.49 \\
0.41 \\
0.44 \\
0.44 \\
0.56 \\
0.43 \\
0.47 \\
0.44 \\
0.44 \\
0.49 \\
0.49 \\
0.44 \\
0.49 \\
0.46 \\
0.41 \\
0.45 \\
0.43 \\
0.43 \\
0.48 \\
0.44 \\
0.42 \\
0.46 \\
0.50 \\
0.43 \\
0.40 \\
0.48 \\
0.48 \\
0.48\end{array}$ & 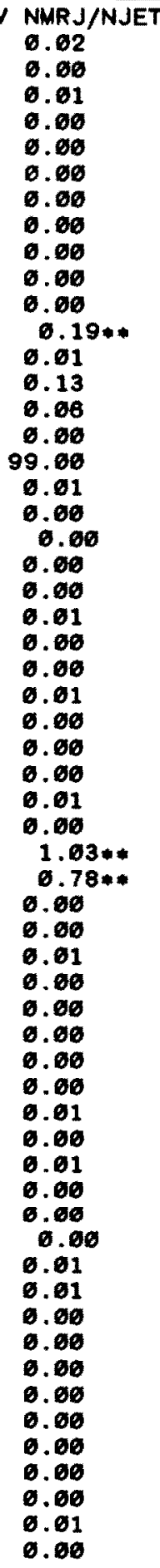 \\
\hline
\end{tabular}


Table 20 Characteristics of runs in the 1987 data (continued).

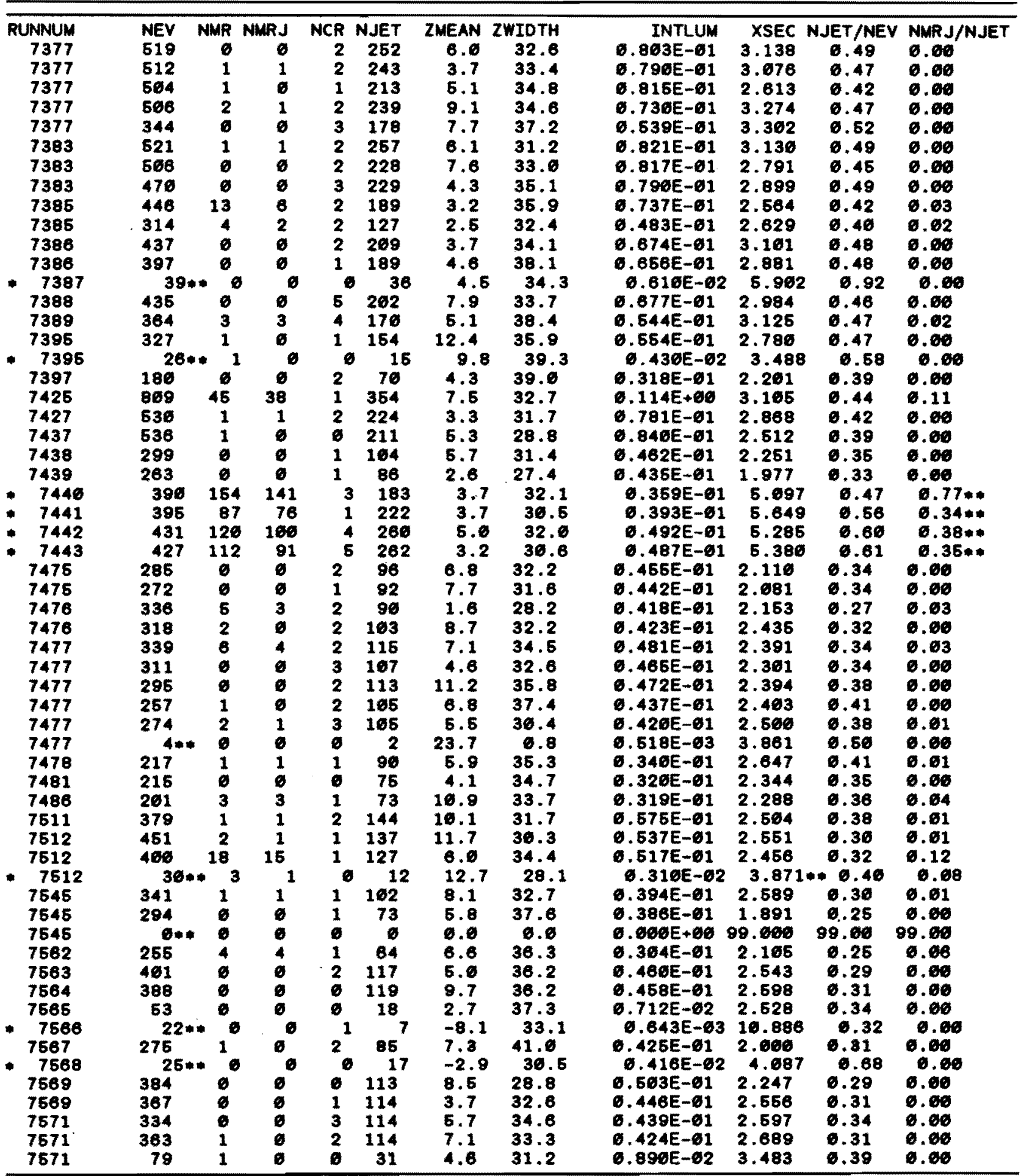


Table 20 Characteristics of runs in the 1987 data (continued).

\begin{tabular}{|c|c|c|c|c|c|c|c|c|c|c|c|}
\hline 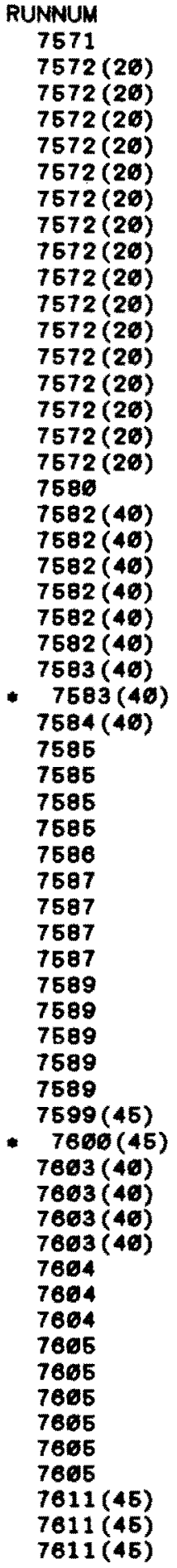 & 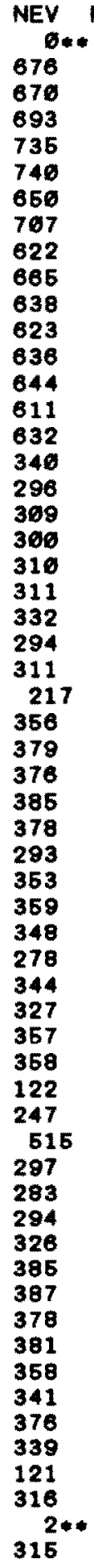 & 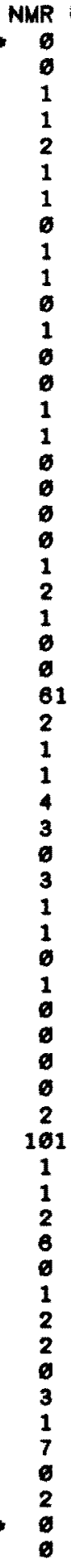 & 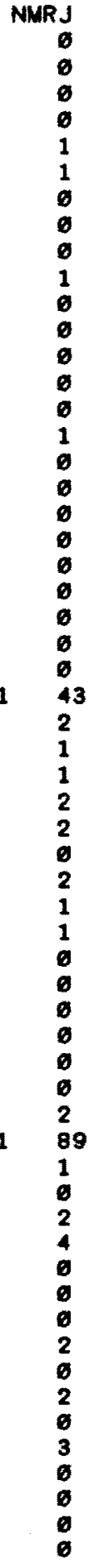 & 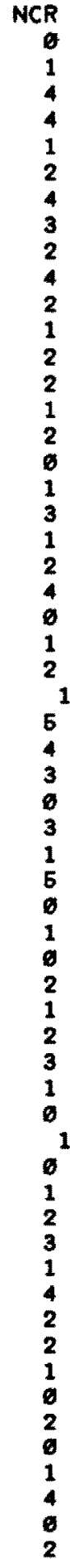 & 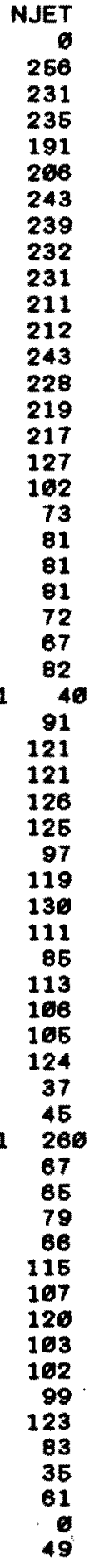 & $\begin{array}{c}\text { ZMEAN } \\
0.6 \\
5.6 \\
4.6 \\
8.7 \\
6.5 \\
6.6 \\
7.5 \\
7.7 \\
5.2 \\
4.3 \\
5.2 \\
4.7 \\
6.3 \\
6.2 \\
3.5 \\
3.1 \\
8.8 \\
3.0 \\
2.0 \\
6.7 \\
2.7 \\
5.0 \\
4.6 \\
7.5 \\
11.8 \\
6.3 \\
7.9 \\
2.6 \\
3.1 \\
-1.3 \\
10.4 \\
9.5 \\
8.4 \\
7.8 \\
6.7 \\
2.1 \\
12.0 .1 \\
4.7 \\
6.8 \\
10.1 \\
4.4 \\
11.5 \\
4.1 \\
8.9 \\
4.6 \\
10.3 \\
3.3 \\
-1.6 \\
4.5 \\
6.0 \\
9.9 \\
16.4 \\
7.2 \\
6.8 \\
8.5 \\
1.5 \\
8.4 \\
0.0 \\
4.0\end{array}$ & $\begin{array}{r}\text { ZWIDTH } \\
6.6 \\
31.8 \\
36.6 \\
37.6 \\
39.5 \\
37.9 \\
38.9 \\
37.6 \\
36.3 \\
33.6 \\
36.5 \\
39.6 \\
37.3 \\
37.9 \\
39.4 \\
37.6 \\
35.4 \\
28.7 \\
32.3 \\
36.4 \\
29.3 \\
28.1 \\
31.8 \\
34.6 \\
29.7 \\
26.3 \\
33.4 \\
36.1 \\
33.8 \\
38.7 \\
39.6 \\
35.4 \\
36.2 \\
38.1 \\
34.6 \\
33.6 \\
36.6 \\
38.6 \\
33.6 \\
38.4 \\
46.9 \\
36.3 \\
29.8 \\
33.7 \\
36.5 \\
36.2 \\
34.6 \\
33.9 \\
32.8 \\
34.3 \\
31.5 \\
46.1 \\
39.4 \\
34.1 \\
41.9 \\
34.7 \\
36.7 \\
0.6 \\
36.6\end{array}$ & 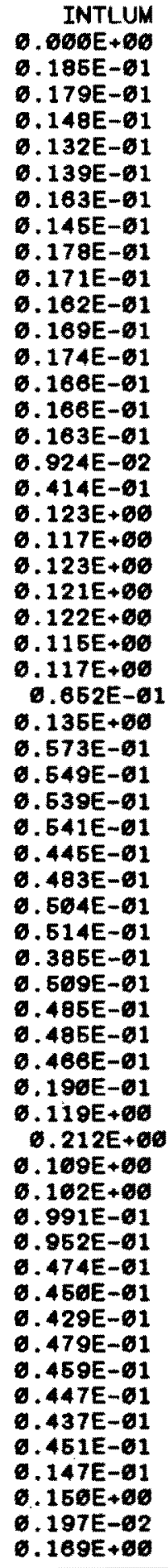 & 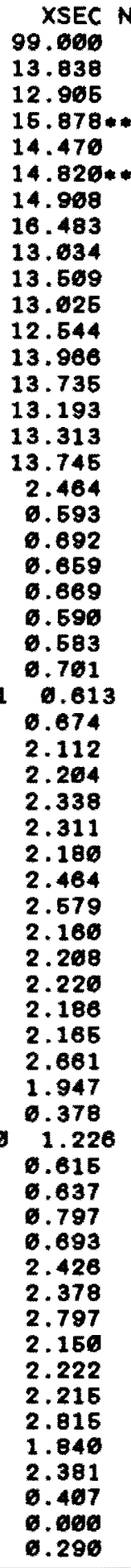 & 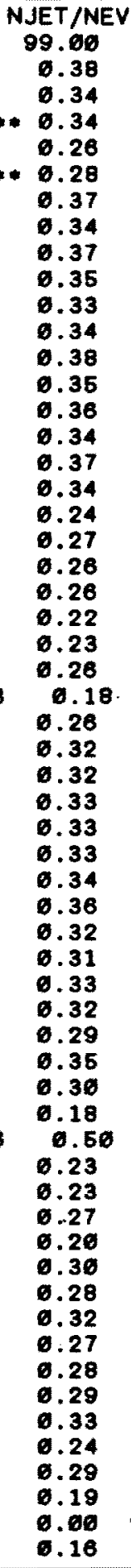 & 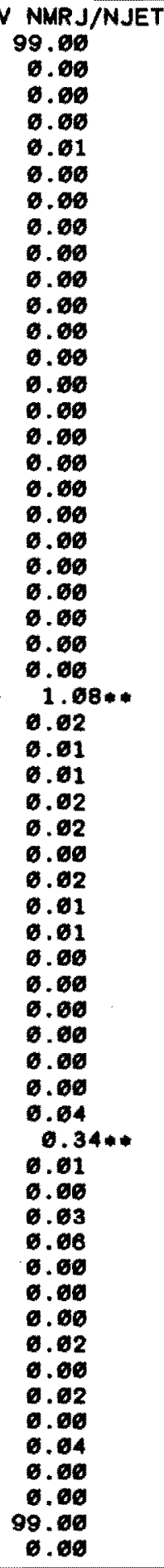 \\
\hline
\end{tabular}


Table 20 Characteristics of runs in the 1987 data (continued).

\begin{tabular}{|c|c|c|c|c|c|c|c|c|c|c|c|}
\hline 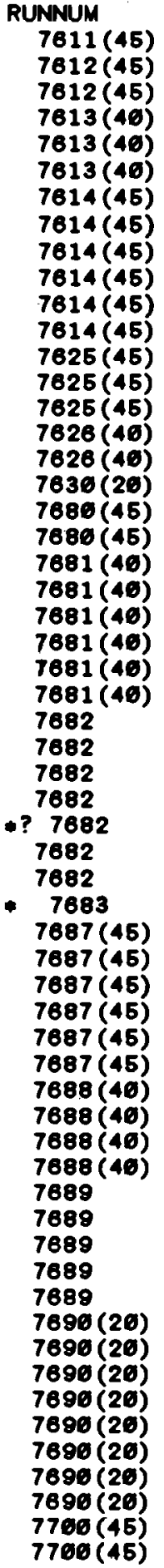 & 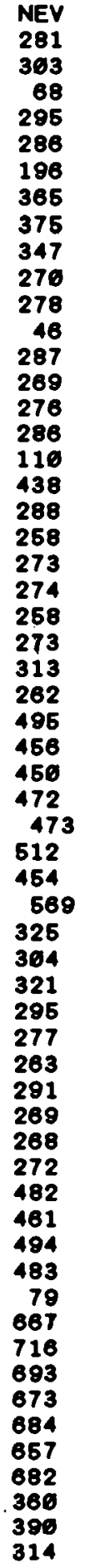 & 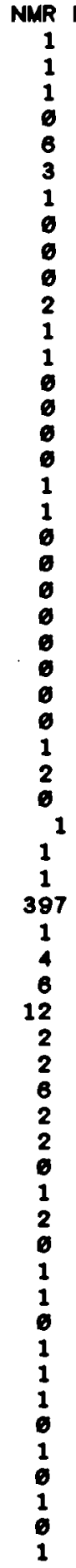 & 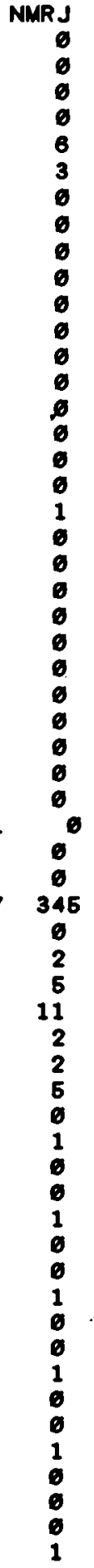 & 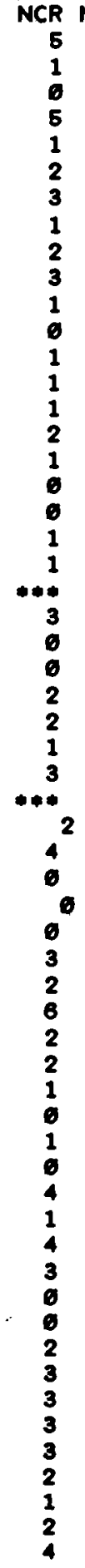 & 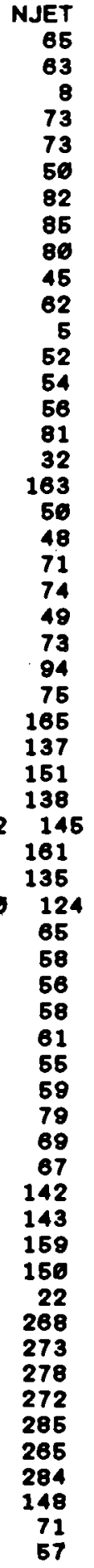 & 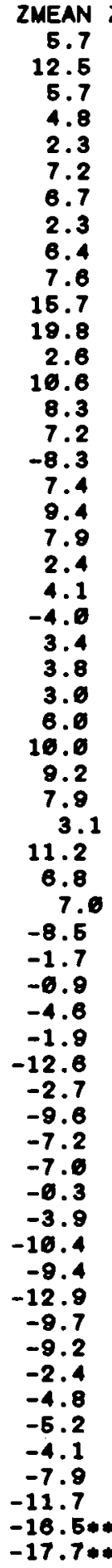 & $\begin{array}{l}\text { 2WIDTH } \\
31.9 \\
39.7 \\
37.7 \\
34.3 \\
31.3 \\
38.4 \\
31.6 \\
32.6 \\
46.1 \\
32.7 \\
34.1 \\
18.1 \\
32.7 \\
28.8 \\
32.6 \\
30.7 \\
30.3 \\
56.5 \\
28.5 \\
33.6 \\
38.9 \\
31.8 \\
31.8 \\
32.8 \\
38.1 \\
32.4 \\
39.0 \\
34.6 \\
35.0 \\
21.6 \\
36.2 \\
46.3 \\
36.0 \\
38.6 \\
26.7 \\
36.0 \\
36.0 \\
31.5 \\
30.1 \\
37.0 \\
24.9 \\
37.1 \\
36.8 \\
32.9 \\
37.8 \\
34.7 \\
37.6 \\
38.8 \\
39.2 \\
38.8 \\
37.9 \\
38.4 \\
34.9 \\
36.2 \\
38.8 \\
36.2 \\
38.6 \\
36\end{array}$ & 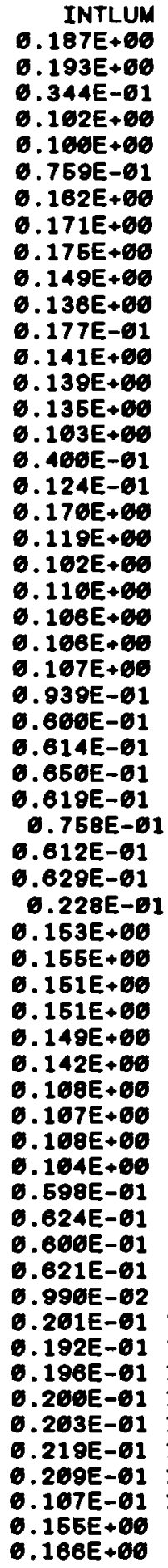 & 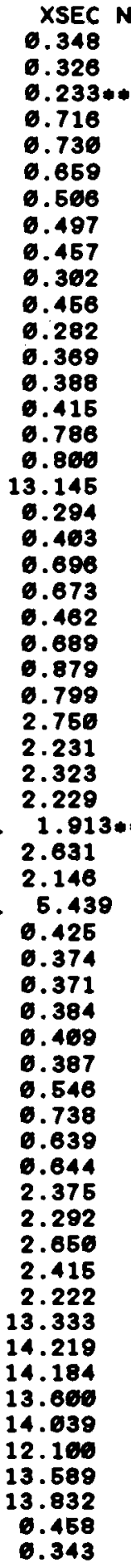 & 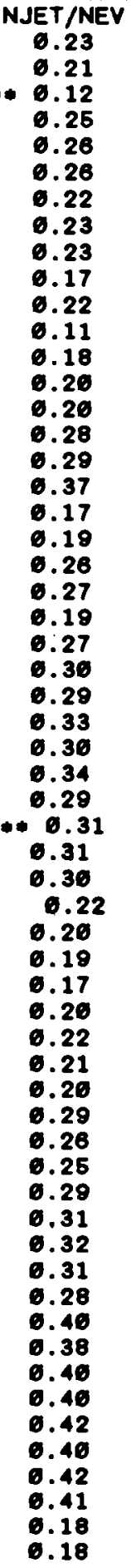 & 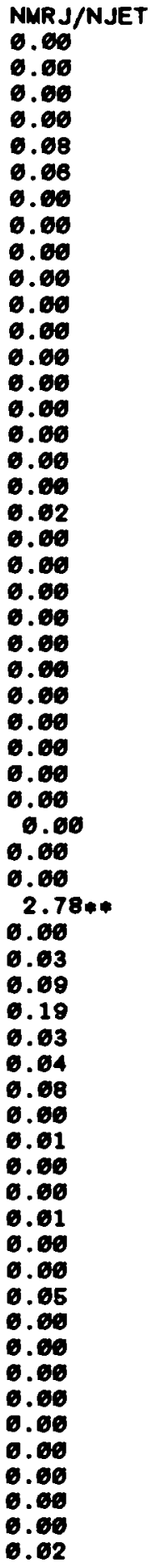 \\
\hline
\end{tabular}


Table 20 Characteristics of runs in the 1987 data (continued).

\begin{tabular}{|c|c|c|c|c|c|c|c|c|c|c|c|}
\hline 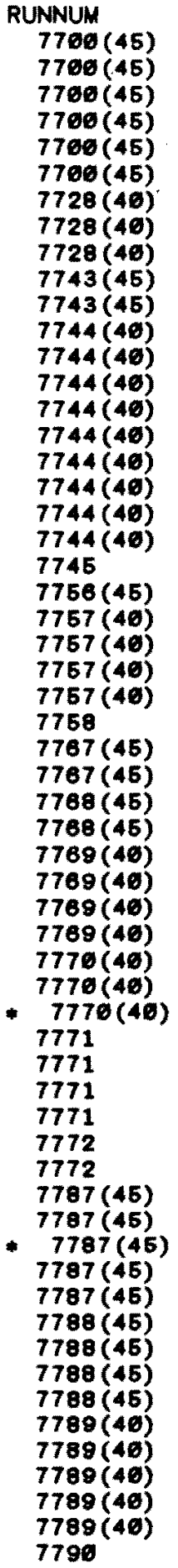 & $\begin{array}{l}\text { NEV } \\
362 \\
368 \\
299 \\
318 \\
282 \\
27 \\
346 \\
315 \\
118 \\
325 \\
339 \\
318 \\
315 \\
316 \\
346 \\
288 \\
341 \\
315 \\
325 \\
365 \\
918 \\
63 \\
332 \\
328 \\
274 \\
271 \\
365 \\
331 \\
246 \\
337 \\
296 \\
324 \\
283 \\
281 \\
318 \\
284 \\
268 \\
372 \\
495 \\
536 \\
485 \\
460 \\
471 \\
103 \\
325 \\
313 \\
328 \\
311 \\
399 \\
362 \\
316 \\
393 \\
319 \\
314 \\
323 \\
314 \\
328 \\
483\end{array}$ & 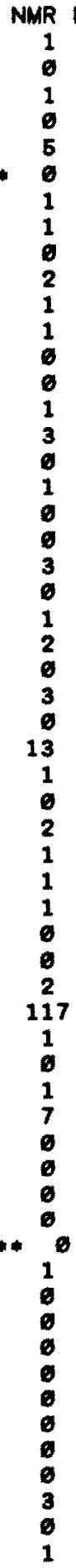 & 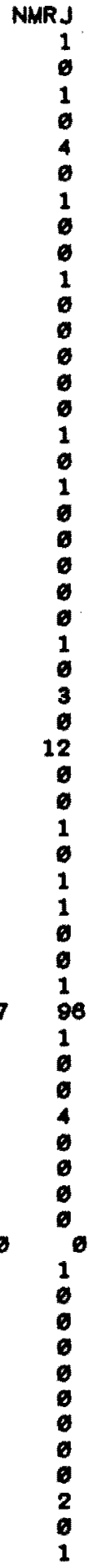 & 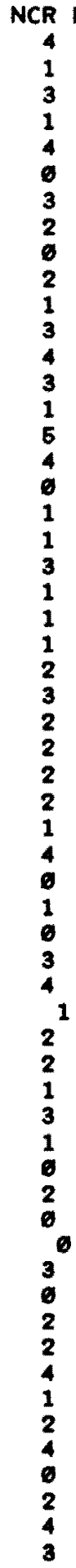 & 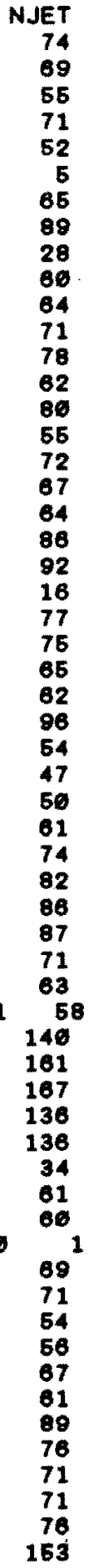 & $\begin{array}{c}\text { ZMEAN Z } \\
-18.7 * \\
-29.1 * \\
-15.3 * \\
-14.5 * \\
-22.6 * \\
-11.5 \\
7.6 \\
2.7 \\
16.4 \\
1.8 \\
3.7 \\
4.9 \\
6.7 \\
-1.4 \\
1.5 \\
-5.7 \\
1.6 \\
4.1 \\
16.5 \\
-6.8 \\
3.2 \\
3.6 \\
-1.4 \\
0.6 \\
6.2 \\
6.3 \\
-6.3 \\
1.3 \\
6.6 \\
7.4 \\
3.9 \\
6.5 \\
2.8 \\
2.9 \\
5.9 \\
4.1 \\
-1.9 \\
-6.4 \\
3.2 \\
3.1 \\
1.6 \\
-2.4 \\
1.6 \\
1.1 \\
4.6 \\
3.9 \\
-51.6 \\
7.8 \\
5.9 \\
3.6 \\
6.0 \\
6.2 \\
4.8 \\
2.2 \\
6.3 \\
5.2 \\
4.8 \\
5.3 \\
1.4\end{array}$ & 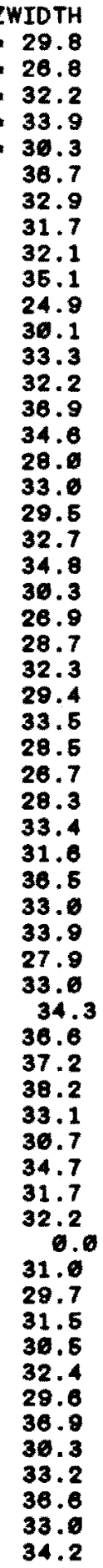 & 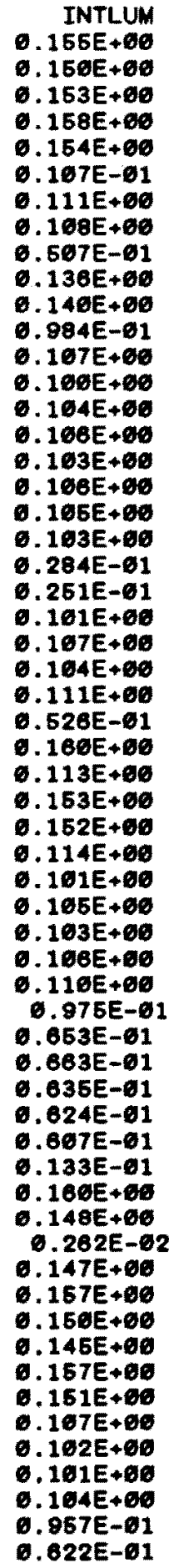 & 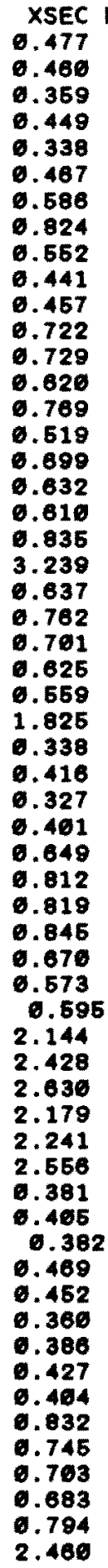 & 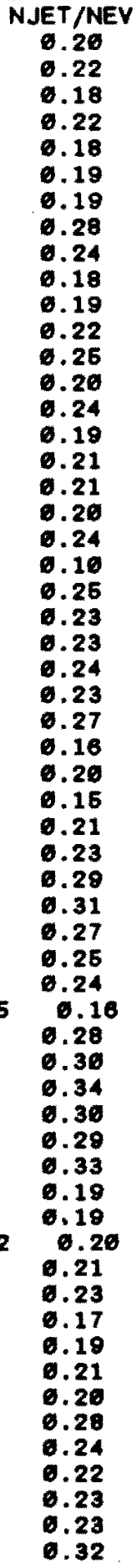 & 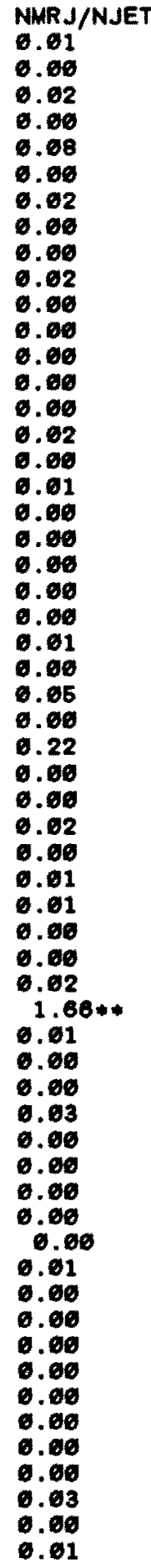 \\
\hline
\end{tabular}


Table 20 Characteristics of runs in the 1987 data (continued).

\begin{tabular}{|c|c|c|c|c|c|c|c|c|c|c|c|}
\hline $\begin{array}{c}\text { RUNNUM } \\
7796 \\
7796 \\
7796 \\
7791 \\
7791 \\
7791 \\
7791 \\
7791 \\
7865(46) \\
7805(4 \theta) \\
7865(46) \\
7865(46) \\
7885(46) \\
7868 \\
7868 \\
7806 \\
7868 \\
7814 \\
7814 \\
7814 \\
7814 \\
7814 \\
7816\end{array}$ & $\begin{array}{l}\text { NEV } \\
566 \\
496 \\
482 \\
565 \\
496 \\
486 \\
464 \\
4 \\
288 \\
296 \\
285 \\
293 \\
288 \\
495 \\
493 \\
472 \\
382 \\
464 \\
479 \\
460 \\
462 \\
462 \\
322\end{array}$ & $\begin{array}{c}\text { NMR } \\
0 \\
6 \\
1 \\
6 \\
0 \\
0 \\
1 \\
0 \\
0 \\
0 \\
0 \\
1 \\
0 \\
1 \\
0 \\
0 \\
0 \\
0 \\
0 \\
0 \\
0 \\
0 \\
0\end{array}$ & $\begin{array}{c}\text { NMRJ } \\
6 \\
0 \\
1 \\
6 \\
0 \\
0 \\
6 \\
6 \\
6 \\
0 \\
0 \\
6 \\
6 \\
6 \\
6 \\
0 \\
0 \\
0 \\
0 \\
0 \\
0\end{array}$ & $\begin{array}{c}\text { NCR } \\
2 \\
3 \\
6 \\
6 \\
1 \\
6 \\
2 \\
6 \\
2 \\
3 \\
2 \\
3 \\
2 \\
2 \\
4 \\
2 \\
1 \\
2 \\
6 \\
4 \\
1 \\
1 \\
5\end{array}$ & $\begin{array}{c}\text { NJET } \\
150 \\
131 \\
141 \\
159 \\
134 \\
148 \\
130 \\
6 \\
66 \\
65 \\
67 \\
65 \\
67 \\
148 \\
146 \\
163 \\
124 \\
130 \\
132 \\
142 \\
146 \\
146 \\
90\end{array}$ & $\begin{array}{c}\text { ZMEAN } \\
-6.2 \\
-1.6 \\
6.5 \\
6.6 \\
2.4 \\
-3.6 \\
4.9 \\
6.6 \\
1.6 \\
-2.6 \\
12.6 \\
-3.3 \\
3.3 \\
4.1 \\
1.8 \\
0.6 \\
5.4 \\
3.8 \\
1.8 \\
-1.0 \\
-1.8 \\
-6.2 \\
2.0\end{array}$ & $\begin{array}{c}\text { ZWITH } \\
31.0 \\
31.5 \\
37.5 \\
36.7 \\
34.3 \\
33.7 \\
34.6 \\
0.6 \\
28.6 \\
33.9 \\
36.6 \\
31.1 \\
36.7 \\
33.5 \\
37.2 \\
36.7 \\
38.1 \\
37.4 \\
36.5 \\
39.8 \\
36.7 \\
34.7 \\
34.2\end{array}$ & 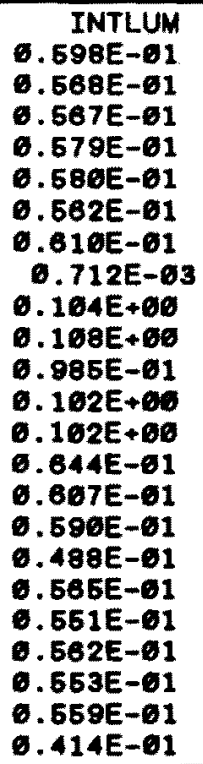 & $\begin{array}{l}\text { XSEC } \\
2.568 \\
2.366 \\
2.487 \\
2.746 \\
2.316 \\
2.633 \\
2.131 \\
6.600 \\
6.636 \\
0.602 \\
6.686 \\
6.637 \\
6.657 \\
2.298 \\
2.389 \\
2.593 \\
2.641 \\
2.361 \\
2.396 \\
2.527 \\
2.532 \\
2.594 \\
2.174\end{array}$ & $\begin{array}{c}\text { NJET/NEV } \\
0.36 \\
6.27 \\
0.29 \\
6.31 \\
0.27 \\
6.36 \\
6.28 \\
0.60 \\
6.23 \\
0.22 \\
6.21 \\
.22 \\
0.23 \\
6.36 \\
0.29 \\
0.32 \\
0.32 \\
.28 \\
0.28 \\
0.31 \\
.31 \\
0.32 \\
0.28\end{array}$ & $\begin{array}{l}\text { NMR J/NJET } \\
0.00 \\
0.60 \\
0.61 \\
0.00 \\
0.60 \\
0.60 \\
0.00 \\
98.60 \\
0.60\end{array}$ \\
\hline
\end{tabular}




\section{REFERENCES}

1. M. Gell-Mann, CTSL-20 (1961).

M. Gell-Mann, Phys. Rev. 125(1962) 1067.

Y. Ne'eman, Nucl. Phys. 26(1961) 222.

2. M. Gell-Mann, Phys. Lett. 8(1964) 214.

G. Zweig, CERN reports TH 401, 412 (1964) (unpublished).

3. M.Y. Han and Y. Nambu, Phys. Rev. $139(1965) B 1006$.

4. See, for example, E. Reya, Phys. Rep. 69(1981)195, section 1 and section 5 (P.222).

5. J.D. Bjorken, Phys. Rev. 179(1969) 1547.

6. The story was told by J.D. Bjorken in Physics Today $42(1989) 56$.

J.D. Bjorken and E.A. Paschos, Phys. Rev. 185(1969) 1975.

J.D. Bjorken and E.A. Paschos, Phys. Rev. D1(1970) 3151 .

7. H. Fritzsch, M. Gell-Mann and H. Leutwyler, Phys. Lett. 47B(1973) 365.

8. H.D. Politzer, Phys. Rev. Lett. $30(1973) 1346$.

D.J. Gross and F. Wilczek, Phys. Rev. Lett. 30 (1973) 1343.

9. S. Coleman and D.J. Gross, Phys. Rev. Lett. 31 (1973)851.

10. G. 't Hooft, Nucl. Phys. B33(1971) 173.

11. M. Gell-Mann and F. Low, Phys. Rev. 95(1954) 1300.

C.G. Callan, Phys. Rev. D2(1970) 1541.

K. Symanzik, Comm. Math. Phys. 18(1970) 227.

12. E. Eichten, I. Hinchliffe, K. Lane, and C. Quigg, Rev. Mod. Phys. $56(1984) 579$.

13. F.E. Paige and S.D. Protopopescu, talk at the XVI International Symposium on Multiparticle Dynamics, Israel, 1985. (Brookhaven National Lab. report BNL-37066, 1985). 
14. E. Eichten, K. Lane. and M.E. Peskin, Phys. Rev. Lett. 50(1983) 811.

15. P.H. Frampton and S.L. Glashow, Phys. Lett. 190B(1987) 157.

16. P.H. Frampton and S.L. Glashow, Phys. Rev. Lett. 58 (1987) 2168.

17. J. Bagger, C. Schmidt and S. King, Phys. Rev. D37(1988) 1188.

18. F. Cuypers and P.H. Frampton, Phys. Rev. Lett. $60(1988) 1237$.

19. R.W. Robinett, Penn. State Univ. report PSU/TH/50 (1988).

20. UA1 Collaboration, Phys. Lett. 209B(1988) 127.

21. CDF Collaboration, Nucl. Instr. and Meth. A271(1988) 387 .

22. L. Balka et al., Nucl. Instr. and Meth. A267(1988) 272.

23. S. Bertolucci et al., Nucl. Instr. and Meth. A267(1988) 301.

24. S. Hahn et al., Nucl. Instr. and Meth. A267(1988)351.

25. Y. Fukui et al., Nucl. Instr. and Meth. A267(1988) 280.

G. Brandenburg et al., Nucl. Instr. and Meth. A267 (1988) 257.

S. Cihangir et al.; Nucl. Instr. and Meth. A267(1988) 249.

26. F. Snider et al., Nucl. Instr. and Meth. A268(1988) 75 .

27. G. Drake et al., Nucl. Instr. and Meth. A269(1988)68.

28 D. Amidei et al., Nucl. Instr. and Meth. A269(1988)51.

29. E. Barsotti et al., Nucl. Instr. and Meth. A269(1988)82.

30. V.E. Barnes, talk at the Eighth Vanderbilt Conference, 1987 (CDF note \%02).

31. Algorithm by A. Sansoni, summarized in CDF note 569 by A. Baden.

32. S. Kuhlmann, CDF note \$581.

G. Redlinger and $Y$. Tsai, CDF note 521 . 
33. P. Hurst and M. Franklin, CDF note $\$ 847$.

34. A. Baden, CDF note $\$ 569$.

35. M. Atac, S. Cihangir and Y. Fukui, CDF note $\$ 544$.

D. Brown and M. Franklin, CDF note \#696.

36. Algorithm by D. Brown.

37. M.G. Albrow et al., Nucl. Phys. B160(1979) 1 and references therein.

A.G. Clark et al., Nucl. Phys. B160(1979) 397 and references therein.

38. UA2 Collaboration, Phys. Lett. 118B(1982) 203.

UA1 Collaboration, Phys. Lett. 123B(1983)115.

39. CDF Jet Group, D. Brown et al., CDF note \#605.

40. S. Bertolucci et al., CDF note \#290.

41. See H. Jensen, CDF note \$532.

42. R. Wigmans, On the Energy Resolution of Uranium and other Hadron Calorimeters, CERN/EF86-18, submitted to Nucl. Instr. and Meth.

43. S. Behrends, J. Huth and S. Kuhlmann, CDF note \$686.

44. S. Behrends and S. Kuhlmann, CDF note \$684.

45. S. Behrends et al., CDF note \#583.

46. R.D. Field and R.P. Feynman, Nucl. Phys. B136(1978)1.

47. UA2 Collaboration, Phys. Lett. 154B(1985) 338.

48. UA2 Collaboration, Phys. Lett. 186B(1987)452.

49. R. St.Denis, Ph.D. thesis, Harvard Univ., December 1988.

50. T.M. Liss, CDF note 552 . 
51. D.W. Duke and J.F. Owens, Phys. Rev. D30(1984) 49.

52. See Ref. 12, P.592.

53. See Ref. 12, P.684.

54. This was pointed out by S. Behrends and J. Huth, CDF note 747. The formula for Poisson $\chi^{2}$ was also suggested.

55. UA1 Collaboration, Phys. Lett. 172B(1986) 461 .

56. UA2 Collaboration, Phys. Lett. 138B(1984) 430. UA2 Collaboration, Phys. Lett. 160B(1985) 349.

57. E. Reya, Phys. Rep. 69 (1981) 195.

58. S. Weinberg, Phys. Rev. 118(1960) 838 .

59. K.G. Wilson, Phys. Rev. $179(1969) 1499$.

60. H. Georgi and H.D. Politzer, Phys. Rev. D9(1974) 416. D.J. Gross and F. Wilczek, Phys. Rev. D9(1974)980

61. G. Altarelli and G. Parisi, Nucl. Phys. B126(1977) 298.

62. L.N. Lipatov, Sov. J. Nucl. Phys. $20(1974) 94$.

63. C.F. Weizsacker, Z. Physik 88(1934)612 E.J. Williams, Phys. Rev. 45(1934) 729.

64. J. Kogut and L. Susskind, Phys. Rev. D9(1974)697.

65. See Yu.L. Dokshitzer, D.I. D'yakonov and S.I. Troyan, Phys. Rep $58(1980) 269$.

66. See, e.g. A.J. Buras, Rev. Mod. Phys. 52(1980) 199 .

67. A. Devoto, D.W. Duke, J.F. Owens and R.G. Roberts, Phys. Rev. D27 (1983) 508 . 
68. A. Ali, E. Pietarinen, G. Kramer and J. Will rodt, Phys. Lett. 93B(1980) 155 .

69. T. Sjostrand, Computer Phys. Comm. 39(1986) 347 .

T. Sjostrand and M. Bengtsson, Computer Phys. Comm. 43(1987) 367 .

70. G.C. Fox and S. Wolfram, Nucl. Phys. B168(1980) 285.

71. R. Odorico, Nucl. Phys. B228(1983)381, Computer Phys. Comm. $32(1984) 139$.

72. R.D. Field and S. Wolfram, Nucl. Phys. B213(1983)65.

73 B.R. Webber, Nucl. Phys. B238(1984) 492.

74. See the references in Ref. 73.

75. T.D. Gottschalk, CALT-68-1241 (1985).

76. T. Sjostrand, FERMILAB-Pub-85/23-T (1985)

77. See T. Sjostrand, talk at the XIXth International Symposium on Multiparticle Dynamics, Arles, France, 1988. LUND report LU TP 88-12 (1988).

78. J. Freeman and M. Eaton, CDF note SIMCALOR.MEM (1985). 